
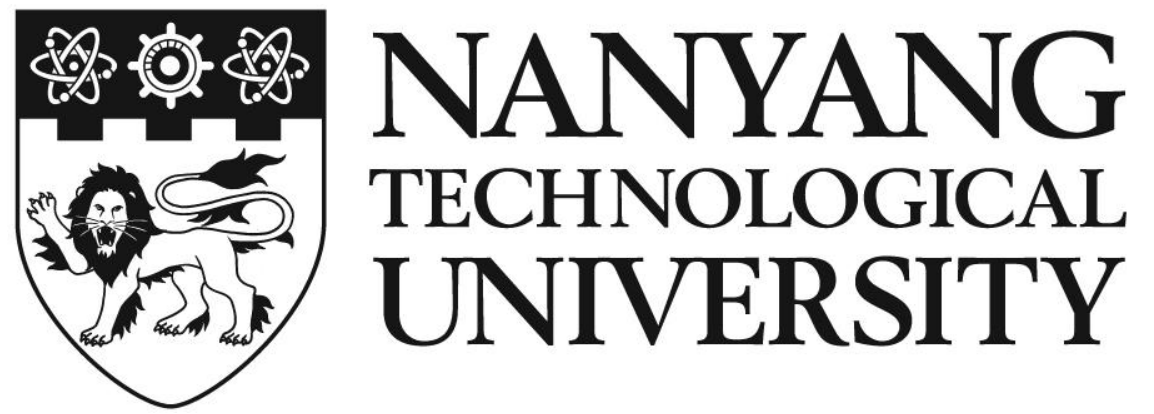

STUDIES ON CONVERSION OF BIOMASS-BASED MATERIALS INTO HMF-DERIVED CHEMICALS 


\section{STUDIES ON CONVERSION OF BIOMASS-BASED MATERIALS INTO HMF-DERIVED CHEMICALS}

\section{ZHAO JUN}

School of Chemical and Biomedical Engineering

A thesis submitted to the Nanyang Technological University in partial fulfillment of the requirement for the degree of Doctor of Philosophy 


\begin{abstract}
The demand for energy and resources of the world now outstrips the earth's ability to meet. With the decreasing of fossil fuel resource and serious environmental pollution caused by the overuse of fossil fuel, biomass energy has attracted more and more attention. As an important solution to the shortage of energy and resources, biorefinery can integrate biomass conversion process to produce energy and chemical building blocks for a diverse range of applications.

Among the various renewable building blocks, 5hydroxymethylfurfural(HMF) plays an important role due to its rich chemistry and potential availability. It can be obtained from monosaccharides such as fructose, glucose, as well as cellulose directly. Moreover, HMF can also be used to produce many valuable molecules such as levulinic acid, dihydroxymethylfuran, 2,5-furandicarboxylic acid, 2,5-diformylfuran (DFF) and 5-hydroxy-4-keto-2-pentenoic acid. Here, the catalytic transformation of fructose to HMF and its derivates are the study focus. Considering the separation of products and reuse of catalysts, heterogeneous reaction system with carbon-based solid catalysts was designed and applied in the conversion of fructose to HMF and DFF.
\end{abstract}

Dehydration of monosaccharides is the main method for the manufacture of HMF. Carbon spheres bearing high stability, low cost and abundant strong protonic acid sites were used as catalyst. The carbon spheres were prepared through a facile hydrothermal method with glucose as the carbon precursor. And then sulfonation treatment was used to increase the amount and strength of the acid sites on the surface of the carbon spheres. The carbon spheres based solid acid showed excellent performance in the dehydration of fructose to HMF. The influences of acid site density, reaction time, solvents, catalysts amount, temperature and mole ratio of catalyst to substrate were investigated. Under 
optimized conditions, $90 \%$ yield of HMF was obtained with $100 \%$ fructose conversion in only $1.5 \mathrm{~h}$ at $160^{\circ} \mathrm{C}$.

DFF, which is an important monomer for industry can be synthesized by the selective oxidation of hydroxyl group of HMF. Vanadium-embedded mesoporous carbon microspheres were synthesized and applied in the aerobic oxidation of HMF to DFF, showing almost $100 \%$ selectivity towards DFF. The catalytic activity of the initially prepared vanadium-embedded carbon solid spheres is not ideal due to the limit contact chance between the reactant and the active site. However, after the introduction of mesoporous structure into the carbon sphere, the activity of the catalyst increased remarkably. But there was a slight decrease of the catalytic activity during the recycling test due to the leaching of the active sites.

Based on the successful synthesis of HMF and DFF through dehydration of fructose and selective oxidation of HMF respectively, molybdenum trioxide supported on carbon spheres were prepared and used as bifunctional catalysts for the one-pot and one-step conversion of fructose to DFF. The catalysts were prepared with a similar method as the vanadium-embedded carbon spheres. Different after-treatment methods were used to modify the physiochemical properties of the catalyst in order to achieve the best DFF yield. As a result, 77\% DFF yield and $100 \%$ fructose conversion can be obtained under optimized conditions.

The component, morphology and structural properties of the catalysts were characterized by many techniques such as X-ray diffraction, Raman spectroscopy, Fourier transform infrared spectroscopy, Element analysis, X-ray absorption near edge structure, Thermo gravimetric analyzer and so on. Different reaction conditions were investigated to reach the highest yield of target product. In addition, the reusability of the catalysts was also tested. 


\section{Acknowledgements}

Time flies so fast these years, but once the special August comes, I remember the sunshine and the rain of the day when I set my foot in NTU for the first time. However, this August comes with not only sunshine and rain but also graduation. As graduation day is approaching, here, I try to concentrate all my appreciations into the following sentences.

First, I would like to show my genuine gratitude to Professor Yanhui Yang, my Ph.D. supervisor. It is impossible for me to finish my PhD without the most valuable guidance and supports from him. Prof. Yang inspired me and encouraged me to overcome the problems and challenges and enhance my research skills.

Secondly, I am very grateful for the valuable instructions, advices and supports from Dr Chunmei Zhou, Dr Kaixin Li, Dr Chao He, Dr Yihu Dai. I also want to thank all my group members because they really gave me a huge help in my research work. They are Dr Yibo Yan, Dr Shuchao Wang, Dr Qian Zhang, Dr Amaniampong Prince Nana, Mr Yong yan, Mr Xiaoping Chen, Mr Jijiang Huang. I need also to show my gratitude to my friends, which give me a lot of happiness in life, and I really cherish the friendship with them. They are $\mathrm{Mr}$ Yiping Zhang, Mr Shunpeng Lv, Mr Tianyu Xiang, Mr Mingshi Xie, Mr Kai Wang.

I have to show gratitude to my parents, they are the people who stand behind me, silently supporting me, encouraging me.

I also have to thank the School of Chemical and Biomedical Engineering, Nanyang Technological University for giving me this chance to pursue my Ph.D.

Last but not least, I need to show my gratitude to all the people who have helped me. I may not list your name in this acknowledgement, I may even don't know your name, but I do think of you often and I will not forget you. 


\section{Contents}

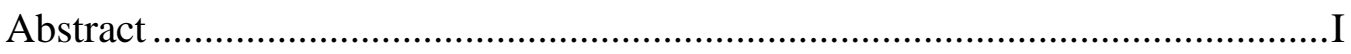

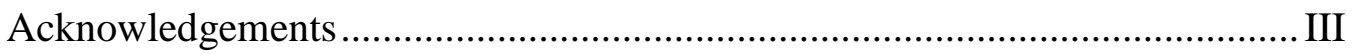

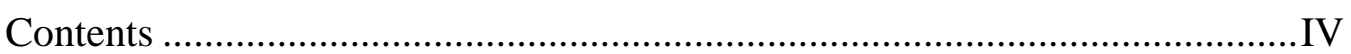

List of Figures......................................................

List of Tables.........................................................

Chapter 1 Background and Philosophy ....................................................... 1

1.1 Background and biomass conversion via heterogeneous catalysis ............ 1

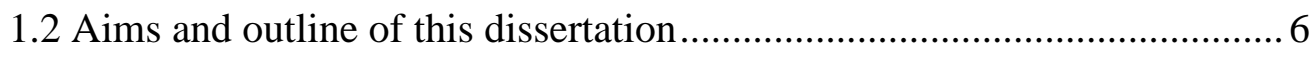

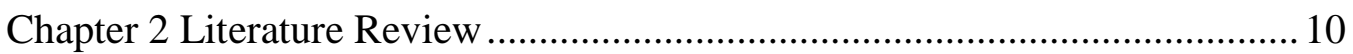

2.1 Synthesis of HMF from carbohydrates ............................................. 10

2.2 Synthesis of 5-hydroxymethylfurfural-based furan derivatives ............... 18

2.3 Synthesis and applications of carbon-based acid catalysts ...................2. 25

2.4 Synthesis and applications of carbon-supported metal catalysts .............32

2.5 Synthesis and applications of carbon sphere catalysts .......................... 38

Chapter 3 Techniques of Characterization, Synthesis, and Catalytic Reactions 44

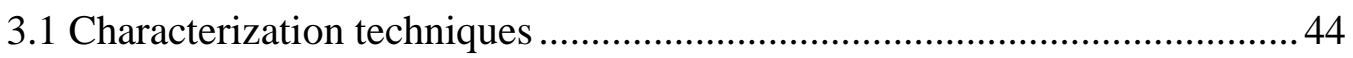

3.1.1 Characterization of catalyst morphologies ..................................... 44

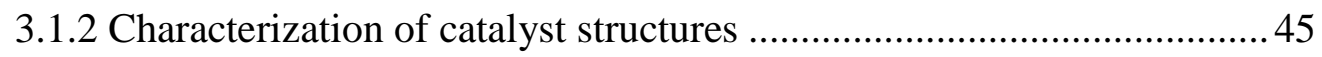

3.1.3 Characterization of catalyst active sites ........................................46

3.1.4 Characterization of chemical properties of catalysts......................... 47

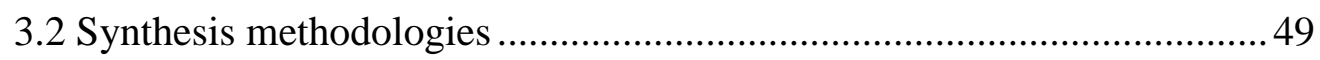

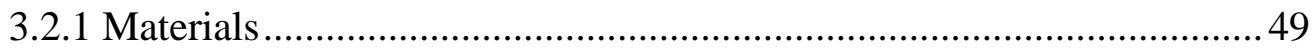

3.2.2 Preparation of sulfonated carbon sphere solid acid catalysts ...............49 
3.2.3 Preparation of V-embedded mesoporous carbon microspheres .50

3.2.4 Preparation of molybdenum trioxide supported on carbon spheres ......50

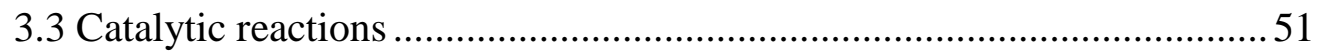

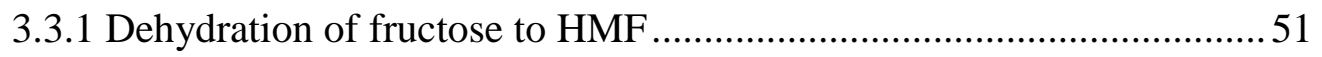

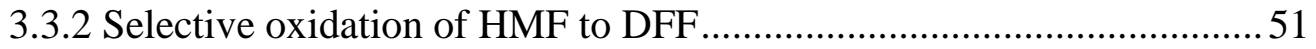

3.3.3 One-pot and One-step synthesis of DFF from fructose........................52

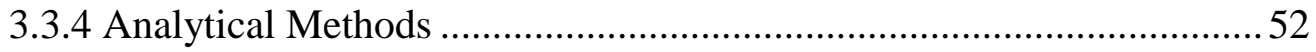

Chapter 4 Efficient Dehydration of Fructose to 5-hydroxymethylfurfural over

Sulfonated Carbon Sphere Solid Acid Catalysts ............................................54

4.1 Characterization of CS catalysts ...................................................54

4.2 Catalytic performance of catalysts ......................................................59

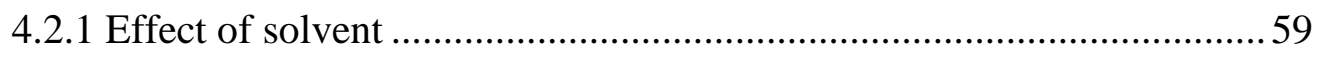

4.2.2 Effect of the acid site density over the catalyst ..................................60

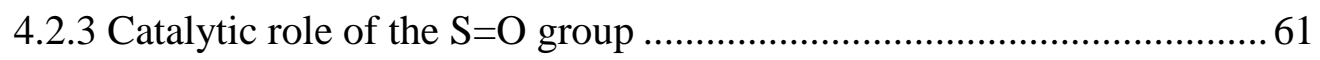

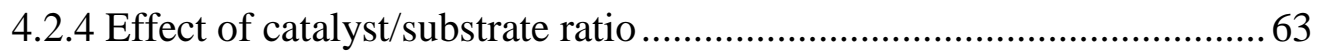

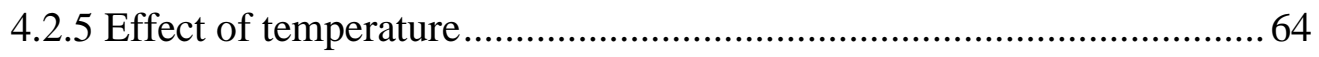

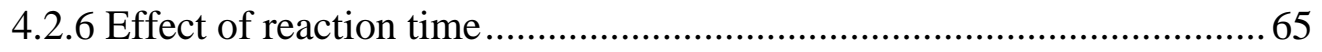

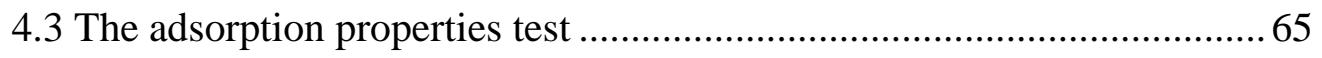

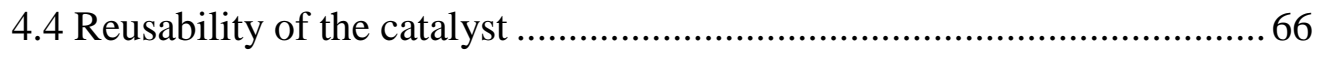

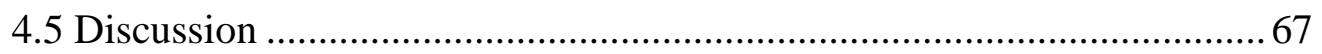

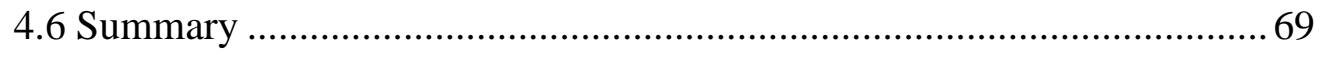

Appendix: Supporting Information ................................................... 70

Chapter 5 Selective Aerobic Oxidation of 5-hydroxymethyl-2-furfural into 2, 5diformylfuran over V-embedded Mesoporous Carbon Microspheres 
5.1 Catalyst characterization of V-embedded mesoporous carbon

microspheres

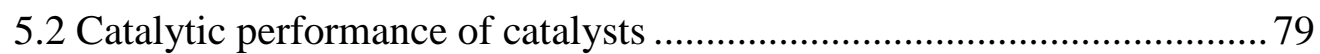

5.2.1 Catalytic oxidation of HMF over V-embedded carbon spheres............79

5.2.2 Effect of solvents on the catalytic oxidation of HMF to DFF.....

5.2.3 Effect of temperature and time on the catalytic oxidation of HMF to

DFF.

5.2.4 Effect of catalyst to substrate ratio on the catalytic oxidation of HMF to

DFF .84

5.3 Catalyst recycling experiments .85

5.4 Summary .86

Chapter 6 Molybdenum Trioxide Supported on Carbon Sphere for One-Step Approach to 2,5-Diformylfuran from Fructose .88

6.1 Catalyst characterization .88

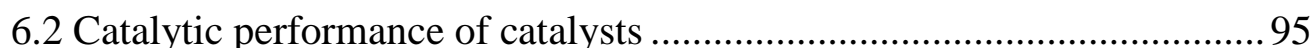

6.2.1 Dehydration of fructose of HMF .95

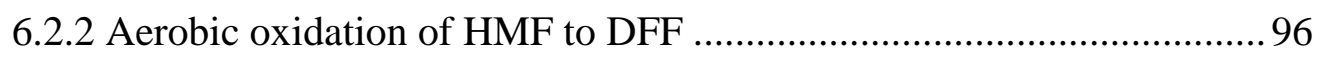

6.2.3 One-pot conversion of fructose to DFF .97

6.3 Optimizations of the reaction conditions over Mo-CS-275 100

6.4 Reusability of the catalyst 101

6.5 Summary 103

Chapter 7 Conclusions and Future Work 105

7.1 Conclusions 105

7.2 Future Work 107 
Reference

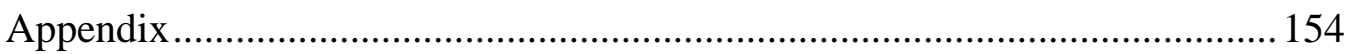




\section{List of Figures}

Scheme 1 Conversion of fructose to HMF and DFF.........................................8

Scheme 2 Conversion of sugars to HMF and other derived chemicals..............11

Fig. 4.1 (a) XRD patterns of CS, CS-1, CS-2, CS-3 and CS-4, (b) Raman

spectra of CS, CS-1, CS-2, CS-3 and CS-4, (c) FTIR spectra of CS, CS-1, CS-2,

CS-3 and CS-4

Fig. 4.2 TPD profiles for carbon spheres. A-C $\mathrm{CO}_{2}$ desorption profiles, D-F CO desorption profiles. A and D are carbon spheres before sulfonation, B and E are carbon spheres after sulfonation, $\mathrm{C}$ and $\mathrm{F}$ are sulfonated carbon spheres recycled after dehydration reaction.

Fig.4.3 FESEM images of: (a) CS, (b) CS-1, (c) CS-2, (d) CS-3, (e) CS-4, (f) C1, (g) C-2, (h) C-3, (i) C-4.

Fig.4.4 Dehydration of fructose in different solvents. Reaction conditions: fructose $(500 \mathrm{mg})$, catalyst $(100 \mathrm{mg})$, solvent $(10 \mathrm{~mL}), 140{ }^{\circ} \mathrm{C}$ for $2 \mathrm{~h}$.

Fig.4.5 Dehydration of fructose by different catalysts. Reaction conditions:

fructose $(500 \mathrm{mg})$, catalyst $(100 \mathrm{mg})$, DMSO $(10 \mathrm{~mL}), 140{ }^{\circ} \mathrm{C}$ for $0.5 \mathrm{~h}$.

Fig.4.6 The influence of ratio of catalyst/substrate on the conversion and HMF selectivity. Reaction conditions: fructose $(500 \mathrm{mg})$, catalyst (following the ratio), DMSO (10 mL), $140{ }^{\circ} \mathrm{C}$ for $0.5 \mathrm{~h}$ .64

Fig.4.7 Influence of reaction time and temperature on the dehydration of fructose to HMF. Reaction conditions: fructose $(500 \mathrm{mg})$, catalyst(100 mg), $\operatorname{DMSO}(10 \mathrm{~mL}),(\mathrm{a}) 100{ }^{\circ} \mathrm{C},(\mathrm{b}) 120^{\circ} \mathrm{C},(\mathrm{c}) 140^{\circ} \mathrm{C}, \quad$ (d) $160{ }^{\circ} \mathrm{C}$ .65

Fig.4.8 Fructose conversions and selectivity towards HMF in the recycling runs. Reaction conditions: fructose (500 mg), catalyst (100 mg), DMSO (10 $\mathrm{mL}), 140^{\circ} \mathrm{C}, 1 \mathrm{~h}$

Fig. S1. Leaching test of sulfonic acid groups on CS acid..............................70

Fig. S2. FESEM pictures of CS-2 before and after reaction..................70

Fig. S3. 1H-NMR spectra of HMF isolated from the reaction mixture .71 
Fig. 5.1 XRD analysis of V-CS before and after thermal treatment.................73

Fig. 5.2 XANES spectra of V-CS before and after thermal treatment...............74

Fig.5.3 Raman spectra of V-CS before and after thermal treatment.................75

Fig. 5.4 FTIR spectra of V-CS before and after thermal treatment..................76

Fig.5.5 FESEM images of (A) V-CS, (B) V-CS-600, (C) V-CS-700, (D) V-CS800, (E) V-CS-900, (F) V-CS-900-Ar.

Fig. 5.6 Comparison of HMF oxidation over V-CS thermal treated at different temperatures. Reaction conditions: $\mathrm{DMSO}(5 \mathrm{~mL}), \mathrm{HMF}(0.05 \mathrm{~mol})$, catalyst (V $1.2 \mathrm{mg}), 120^{\circ} \mathrm{C}, \mathrm{O}_{2}(20 \mathrm{~mL} / \mathrm{min}), 4 \mathrm{~h}$.

Fig.5.7 Effect of temperature and time on the catalytic oxidation of HMF to DFF. Reaction conditions: DMSO(5mL), HMF(0.05mol), V-CS-800(35mg), $\mathrm{O}_{2}\left(20 \mathrm{~mL} / \mathrm{min}\right.$ ), reaction temperature (A) $100{ }^{\circ} \mathrm{C}$, (B) $120{ }^{\circ} \mathrm{C}$, (C) $140{ }^{\circ} \mathrm{C}$,(D) $160{ }^{\circ} \mathrm{C}$

Fig.5.8 Effect of catalyst to substrate ratio on the catalytic oxidation of HMF to DFF. Reaction conditions: $\mathrm{DMSO}(5 \mathrm{~mL}), \operatorname{HMF}(0.05 \mathrm{~mol}), 120^{\circ} \mathrm{C}$,

$\mathrm{O}_{2}(20 \mathrm{~mL} / \mathrm{min}), 9 \mathrm{~h}$ 85

Fig. 5.9 Catalyst V-CS-800 recycling. Reaction conditions: Solvent (5mL), HMF (0.05mmol), V-CS-800(65mg), $120^{\circ} \mathrm{C}, \mathrm{O}_{2}(20 \mathrm{~mL} / \mathrm{min}), 9 \mathrm{~h} .$. .86

Fig.6.1 FESEM images of Mo-CS (a,b), Mo-CS-275 (c,d), Mo-CS-N2 (e,f)...89

Fig. 6. 2 XRD analysis of Mo-CS, Mo-CS-275 and Mo-CS-N2 ................90

Fig. 6.3 Raman spectrum of Mo-CS, Mo-CS-275, Mo-CS-N2 .......................91

Fig. 6.4 FTIR spectra of Mo-CS, Mo-CS-275 and Mo-CS-N2 .......................92

Fig. 6.5 NH3-TPD curves of Mo-CS, Mo-CS-275 and Mo-CS-N2 ...........94

Fig. 6.6 Dehydration of fructose to HMF over Mo-CS, Mo-CS-275 and Mo-CSN2. Reaction conditions: fructose (200mg), catalyst (30mg), DMSO (5mL), $110^{\circ} \mathrm{C}$ .96 
Fig. 6.7 Aerobic oxidation of HMF to DFF over Mo-CS, Mo-CS-275 and MoCS-N2. Reaction conditions: HMF (63mg, 0.5mmol), catalyst (30mg), $\mathrm{O}_{2}(20 \mathrm{~mL} / \mathrm{min}), \mathrm{DMSO}(5 \mathrm{~mL}) 140{ }^{\circ} \mathrm{C}$ .97

Fig. 6.8 Direct transformation of fructose to DFF catalyzed by Mo-CS, Mo-CS275 and Mo-CS-N2. Reaction conditions: fructose (200mg), catalyst (Mo 2mg), $\operatorname{DMSO}(5 \mathrm{~mL}), \mathrm{O}_{2}(20 \mathrm{~mL} / \mathrm{min}), 130^{\circ} \mathrm{C}, 8 \mathrm{~h}$ .98

Fig. 6.9 Conversion of fructose to HMF and DFF as a function of time. Reaction conditions: fructose (200mg), CS-Mo-275 (30mg), DMSO (5mL), $\mathrm{O}_{2}$ (20mL/min), $130{ }^{\circ} \mathrm{C}$ .99

Fig. 6.10 Influence of ratio of Mo/fructose on the synthesis of HMF and DFF from fructose. Reaction conditions: CS-Mo-275 (30mg), DMSO (5mL), $\mathrm{O}_{2}$ (20mL/min), $130^{\circ} \mathrm{C}$, 100

Fig. 6.11 Reusability test of CS-Mo-275. Reaction conditions: fructose (200mg), CS-Mo-275 (30mg), DMSO (5mL), O 2 (20mL/min), $155^{\circ} \mathrm{C}, 2 \mathrm{~h} . . .102$ Fig. 6.12 XRD and FESEM picture of recovered Mo-CS-275. .103 


\section{List of Tables}

Table 4.1 Relative concentration of functional groups over the CS catalysts....56

Table 4.2 The physicochemical properties of the catalysts..............................58

Table 4.3 The catalytic reaction results for the dehydration of fructose to HMF

with different acid catalysts and salts........................................................62

Table 4.4 The adsorption properties of fructose or HMF by CS................66

Table S1 Element analysis of CS catalyst before and after reaction.................70

Table 5.1. Elemental composition of V-CS before and after thermal

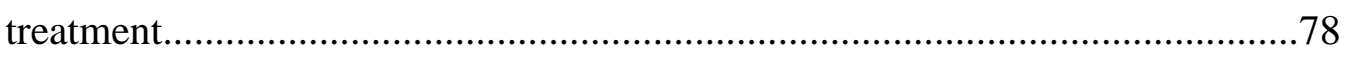

Table 5.2. Textual properties of V-CS before and after thermal

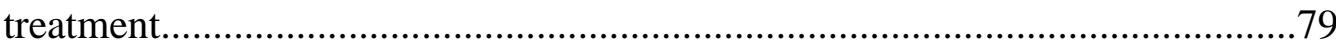

Table 5.3 Effects of solvents on the aerobic oxidation of HMF to

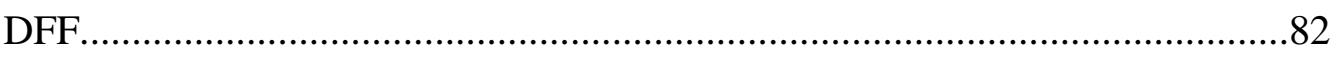

Table 6.1 Elemental composition and surface areas of Mo-CS, Mo-CS-275 and Mo-CS-N2 ....................................................... 93

Table 6.2 Influence of temperature and time on the synthesis of HMF and DFF from fructose 


\section{Chapter 1 Background and Philosophy}

\subsection{Background and biomass conversion via heterogeneous catalysis}

Energy is needed for the survival of human society and is important in developing global economy and improving living standards; thus setting the pace of socioeconomic development[1]. With the development of global economy, the demand for energy is increasing rapidly, while the common energy sources like fossil fuels ( namely oil and gas) are non-renewable, resulting in their long term supply crisis and high prices[2, 3]. However, this is only one side of the coin. On the other hand, the long history of man's misuse of oil and gas has led to some drastic changes on our environment[4]. The air pollution and water pollution can directly affect human health, and the global warming caused by the overproduction of carbon dioxide formed by burning of these non-conventional sources of energy has brought severe calamity to the creatures living on this planet including mankind[5-7]. Under the pressure from the shortages of energy resources and the serious environmental pollution, which even poses a great threat to man's existence and development, people from all around the world are trying to search and develop new, clean and safe energy resources to reduce the dependency on fossil fuels[8, 9]. Renewable energy sources like solar energy, wind energy, tidal energy, geothermal energy, nuclear energy etc. are increasingly attracting people's attention, and countries around the world are taking active measures to push the development of technologies in using these energies [10-12].

Among all the new types of energy resources, biomass energy is one of the most important energy sources to be benefited from in the future. Biomass is a form or carrier of solar energy. Solar energy harnessed by plants for photosynthesis is stored as chemical energy in biomass; it is in fact an effective and cost-effective way of utilising the infinite potential of solar energy[13-15]. Biomass is the most widely spread material on earth including all the animals, 
plants and microorganisms and other derived organic materials. The energy that a plant uses during photosynthesis is $0.2 \%$ of the radiation of the solar energy reaching earth[16]. The absolute value of this energy is very huge, about forty times of the total energies consumed by humans worldwide. As the main ingredients of biomass, about 16 billion tons of lignin and cellulose can be regenerated every year, which is equal to 15-20 times of the oil production[17]. Besides, biomass comes from the carbon dioxide in the air and water, so even if the biomass was burnt to produce heat directly, it would not intensify the greenhouse effect. Replacing the traditional energy sources with biomass can reduce the dependence of coal, oil and gas and environment pollution as well. Generally, the abundant existence and renewability can guarantee the sustainable use of biomass; it's use reducing the carbon dioxide, sulphide and nitrous oxide level in atmosphere, thus making biomass an environment friendly energy source[18]. Biomass is easy to transport and the product quality is stable. In one word, biomass energy is the only storable and transportable renewable energy, and also the most promising substitute for conventional energy sources to realize the utilization on a large scale. But it comes with its own disadvantages such as dispersed distribution, low energy density, low calorific efficiency and complexity of the components.

Actually, biomass materials have been used as an energy source from time immemorial. Most of the time, they are burnt to provide heat energy. Until now, many farmers still rely on biomass for cooking and heating in many rural areas[18, 19]. This kind of unscientific use of biomass material can pollute the environment and waste energy sources. Therefore, it is necessary to develop new technical and scientific lines for systematically utilizing biomass materials.

As an energy source, biomass can either be used directly via combustion to produce heat or indirectly after converting it to various forms of biofuel. 
Historically, humans have harnessed biomass derived energy since the time when people began burning wood to make fire, which should be replaced by new efficient and harmless utilization techniques. Biofuels are classified into two major categories based on the source of biomass. First generation biofuels are derived from sources such as sugarcane and corn starch etc.[20]. Second generation biofuels on the other hand utilize non-food based biomass sources such as agriculture and municipal waste[21, 22]. Despite being the favored alternatives, economical production of second generation biofuel is not yet achieved due to technological issues due to the chemical resitance and structural rigidity of lignocellulosic biomass.

Conversion of biomass to biofuel can be achieved by different methods which are broadly classified into: thermal, chemical and biochemical methods. The thermal methods include biomass gasification technology, thermal cracking technology and biomass liquefaction technology. Biomass gasification technology is a process in which the transfer of the solid biomass into gas fuel happens through thermal chemical reactions[23]. It has a history of more than 100 years, the initial gasification reactor being set up in the year 1883, which used charcoal as raw material to produce gas to drive the internal combustion engine. According to the gasification temperature, biomass gasification can be classified into: room temperature gasification and high temperature gasification[24]. The room temperature gasification includes air gasification, oxygen gasification, steam gasification, steam-oxygen mixture gasification and hydrogen gasification. Usually, the efficiency of room temperature gasification is lower than high temperature gasification which occurs at $1000^{\circ} \mathrm{C}$, where air is mixed with $10-20 \%$ steam as the gas intermediate. The pyrolysis of biomass is also one of the most promising ways of using these renewable forms of future energy, also known as liquefaction technology[25]. Organic molecules with larger molecular weight can be broken down into small molecular with relatively 
fewer carbon atoms. Through the control of reaction temperature, reaction time, heating rate and active gas, different kinds of chemicals can be produced. The pyrolysis conducted under $400^{\circ} \mathrm{C}$ mainly produces coke while the main product becomes gas if the temperature is higher than $500^{\circ} \mathrm{C}[26]$. Biomass liquefaction technology involves the transfer of biomass into liquid hydrocarbon under the conditions of high pressure $(15 \mathrm{MPa})$ and low temperature $\left(250-400^{\circ} \mathrm{C}[27]\right.$. It can be classified into direct liquefaction and indirect liquefaction.

The biochemical methods of transformation of biomass include anaerobic fermentation technology, hydrolysis fermentation technology and biological hydrogen production technology. In the anaerobic environment, the biomass in fermentation tank is first converted to organic acid, alcohol, hydrogen and carbon dioxide by the bacterial action, then the organic acid and alcohol are metabolized to acetic acid and hydrogen, followed by the treatment with methanogens to produce methane. The hydrolysis fermentation technology was used to produce ethanol from sugar, starch and cellulose[27, 28]. First, the crushed cellulose was converted to sugar by catalyzing enzyme. Second, the sugar was converted to ethanol by leavening agent, but the concentration of ethanol was between 10$15 \%$. Distillation and purification is essential to get $95 \%$ volume fraction ethanol. Algae and cyanobacteria can be used to produce hydrogen from biomass which is called biological hydrogen production[29].

Biomass can be transformed to to produce chemical that is more conveniently used, transported or stored, or to exploit some property of the process itself. For example, cellulose can be converted to intermediate platform chemical such sorbitol, glucose, hydroxymethylfurfural etc., and these chemicals are then further transformed to produce hydrogen or hydrocarbon fuels[30, 31]. The commercial production of wood sugars for ethanol production was first considered at the beginning of 20th century. Lignocellulose, a very abundant 
material, are present as important polymers (cellulose, hemicellulose and lignin), of which cellulose and hemicellulose in particular are of high importance, since they are formed from monomers of glucose and they can be used as a carbon source in fermentation process for the production of ethanol[32, 33]. In order to produce industrial chemicals from biomass, it is important to find a range of chemical building blocks derived from renewable resources. One of these, 5hydroxymethylfurfural(HMF), plays an important role, because it can be obtained not only from fructose but also from glucose as well as directly from cellulose. HMF is very useful not only as intermediate for the production of the biofuel dimethylfuran and other molecules, but also for important molecules such as levulinic acid, 2,5-furandicarboxylic acid(FDA), 2,5-diformylfuran(DFF), dihydroxymethylfuran and 5-hydroxy-4-keto-2-pentenoic acid[34-38].

In recent years, many kinds of sugars have been converted to value-added chemicals and it is of great significance. Hexoses are the six-carbon carbohydrates and they exist abundantly in nature. Among them, D-fructose and glucose are suitable to be used as the chemical feedstock[39]. The process of transformation of hexoses into furans and furan derivatives include many steps of reaction such as dehydration, hydrolysis, isomerization, reforming, aldol condensation, hydrogenation and oxidation[40, 41]. In the chemical conversion processes, the compounds like 5-HMF, FDCA, DFF and DMF are interrelated by reaction network. FDCA has been found useful as a fungicide, corrosion inhibitor and melting agent for foundry sands as well as an intermediate in pharmaceutical and photography fields and DFF is applied in the synthesis of special polymers as monomers are formed by the complete or partial oxidation of $\operatorname{HMF}[42,43]$. DFF is also used in the preparation of macrocyclic ligands, as intermediates of pharmaceuticals, as antifungal agents and as a cross-linking agent for poly(vinyl alcohol)[44]. 
Here, we focus on the chemical technologies for the utilization of biomass materials to enable the biomass materials to be converted into many kinds of value added chemicals through chemical reactions. As known to all, catalysts play a very important role during the reactions. Since the Swedish chemist Berzelius discovered a new force which was later called catalysis in 1835 , the development of catalysis science and technology significantly promoted the progress of petroleum industry[45]. Various catalysts have been developed and applied in energy production, environment protection and agricultural production. In a homogeneous reaction system, the reactants and catalysts form a homogeneous phase, there is no phase boundary in the reaction, while in a heterogeneous reaction system, there exist multiple phase reactions, including gas-solid reaction, gas-liquid reaction, liquid-liquid reaction, liquid-solid reaction, gas-liquid-solid reaction and solid-solid reaction. Both of the mentioned catalytic reaction systems have advantages and disadvantages. The most obvious difference between the systems is that it is much easier to separate the catalysts from a heterogeneous reaction system than from the homogenous reaction system. Generally, the separation of catalysts is a cumbersome and energy-intensive process[46], which makes heterogeneous catalysts more popular in industry. Therefore, in order to realize a low-energy way for the reuse of catalyst, we will focus on the development of heterogeneous catalysts for the conversion of carbohydrates to value added chemicals.

\subsection{Aims and outline of this dissertation}

Synthesis of HMF and DFF from fructose was selected as the target reactions for investigation in this Ph.D. study, intending to understand and design optimal heterogeneous catalysts for specific reactions. The activity and selectivity of the catalysts were studied, and the properties of the catalysts were investigated through a series of characterizations. 
The specific objectives of this research are:

A. Design and prepare carbon based solid acids for the dehydration of fructose to $\mathrm{HMF}$.

B. Design and prepare catalysts for aerobic oxidation of HMF to DFF.

C. Design and prepare bifunctional catalysts for one-pot synthesis of DFF from fructose.

D. Investigate the properties of the catalysts through a series characterizations and optimize the reaction conditions to enhance the products yield.

The reaction consists of two steps: the dehydration of fructose to HMF and the aerobic oxidation of HMF to DFF (Scheme 1). The dehydration of fructose to HMF is an acid catalyzed reaction. Organic acids, inorganic acids, salts, Lewis acid and solid acids can be used in the dehydration of carbohydrates as catalysts in different reaction systems[47]. However, homogeneous reaction system has disadvantages in terms of separation and recycling while using solid acid catalysts can avoid these drawbacks. As for the oxidation of HMF to DFF, various catalysts have been prepared and tested with different kinds of oxidants such as $\mathrm{NaOCl}, \quad \mathrm{BaMnO}_{4}, \mathrm{~Pb}(\mathrm{OAc})_{4}$-pyridine, trimethyl ammonium chlorochromate, oxalyl chloride, 2,2,6,6-tetramethylpiperidine-1-oxide and pyridinium chlorochromate[48, 49]. In order to save energy and reduce production costs, one-step transformation of fructose to DFF has attracted a lot of attention. However, at present the one-pot transformation of fructose to DFF is usually processed in two steps with acid catalysts for the dehydration of fructose to $\mathrm{HMF}$ and oxidative catalysts for the oxidation of HMF to DFF. What's more, the oxidative catalysts have to be added in to the system after the complement of the first step to prevent the direct decomposition of fructose to small organic acids. Thus it is still quite challenging to prepare bifunctional catalyst with both acid sites and oxidative sites for the direct synthesis process. 
The wide variety of catalysts and catalysis systems designed and used for biomass conversion will be discussed in Chapter 2 .

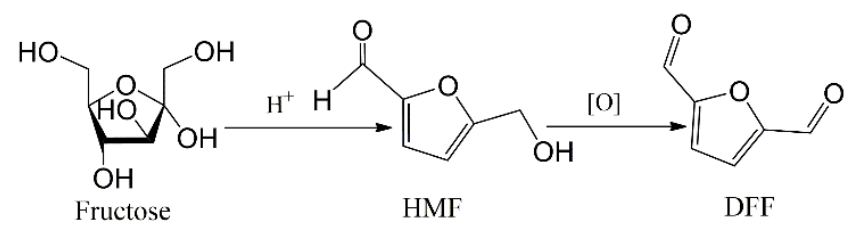

Scheme 1 Conversion of fructose to HMF and DFF

Carbon-based solid acid was used as the catalyst for the dehydration of fructose to HMF in this thesis. Carbon-based solid acid is environment friendly and is insoluble in common organic solvents, and is widely used as supports to load various metal or metal oxide to from carbon composites and applied in catalysis. Here, we prepared carbon spheres by hydrothermal method using carbohydrates as starting materials. The as-synthesized carbon sphere can be easily modified with various functional groups to meet the requirements of the reaction. More specifically, we modified the carbon spheres with $-\mathrm{SO}_{3} \mathrm{H}$ to enhance its acidity to meet the requirements of dehydration of fructose to HMF. These results will be presented in detail in Chapter 4.

The work we have done on the oxidation of HMF to DFF will be presented in Chapter 5. As we mentioned above that carbon materials are widely used as supports to load various metal or metal oxide, vanadium doped carbon spheres were synthesized and applied in the aerobic oxidation of HMF to DFF in this thesis. Metal oxide or supported noble metal such as $\mathrm{Pt} / \mathrm{C}, \mathrm{Pd} / \mathrm{C}$ and $\mathrm{Ru} / \mathrm{C}$ are usually used as catalysts for the aerobic oxidation of HMF to DFF[50-52]. The reaction results are significantly influenced by the reaction conditions such as the temperature, ratio of reactant to catalyst, the reaction time and solvents. Generally, higher temperature and longer reaction time can lead to a higher conversion of reactant and higher yield of target product if it is stable under the 
reaction conditions. Though a lot of catalysts have been developed for the oxidation of HMF to DFF, it is still quite a challenge to produce DFF in high yield, because the aldehyde groups can be further oxidized to carboxyl groups.

Investigation on the one-pot conversion of fructose to DFF will be presented in Chapter 6. DFF, as a valuable intermediate, a highly potential chemical used in the production of furanic polymers and renewable furan urea resin, has not been able to achieve industrial production. One of the reasons behind this is that the separation and purification of HMF is a high energy consuming process, resulting in the high price of HMF and the high cost for production of DFF from HMF. Therefore, to produce DFF from sugars directly without the separation and purification of HMF will be a greener and more economical way. A lot of efforts have been made to realize a one-pot synthesis of DFF from fructose with the combination of the acid catalyst and oxidative catalyst. Based on this idea, molybdenum trioxide supported on carbon spheres were prepared and applied in the one-step conversion of fructose to DFF in this thesis.

All the synthesized catalysts were characterized using a series of characterization techniques, e.g., field emission scanning electron microscope (FESEM) and X-ray diffraction (XRD), RAMAN spectra, Fourier transmission infrared spectroscopy (FTIR), X-ray absorption fine structure (XAFS), Element analysis (EA) and thermal gravimetric analysis (TGA), $\mathrm{NH}_{3} / \mathrm{CO}_{2} / \mathrm{CO}$ temperature programmed desorption (TPD). This part will be presented in Chapter 3. 


\section{Chapter 2 Literature Review}

During the last few decades, the conversion of biomass via platform molecules or building blocks to biomass-based value added chemicals has attracted sharply increasing interests. Among the considerable range of renewable building blocks derived from biomass materials, HMF is regarded as an important intermediate due to its rich chemical properties and the possibility of its easily available synthesis strategy from fructose, glucose, sucrose and even directly from cellulose[53-56]. In this process, catalysts play an important role in activating the reaction and promoting the yield of the target products and product quality. A significant issue in the dehydration of sugars is the formation of humins, which are generally undesirable for chemical purposes. Therefore, developing new catalysts with high surface area and catalytic activity, which can solve the problems of mass transfer resistance, time consumption and fast deactivation commonly faced by heterogeneous catalysts, has attained a great deal of attention. So far, considerable efforts have been made on the catalytic transformation of biomass materials into HMF and HMF derived chemicals[57, 58]. The principles for designing heterogeneous catalyst applied in carbohydrates conversion will be discussed and the advances in the strategies for biomass conversion are also reviewed in this chapter.

\subsection{Synthesis of HMF from carbohydrates}




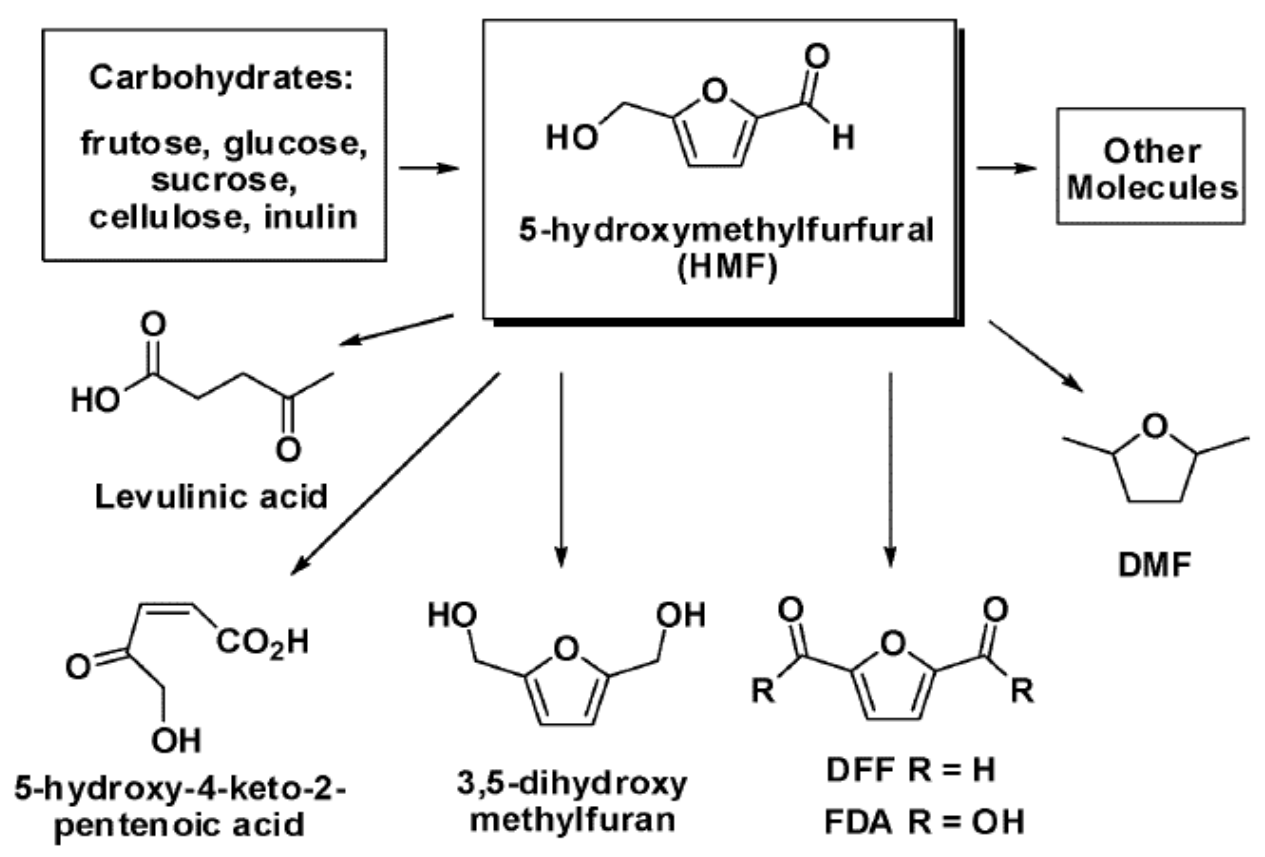

Scheme 2 Conversion of sugars to HMF and other derived chemicals.

As shown in Scheme 2, HMF is a versatile and multi-functional compound and a good starting point for the synthesis of precursors of pharmaceuticals, thermoresistant polymers, and macrocyclic compounds and particularly for the synthesis of dialdehydes, ethers, amino alcohols, and other organic intermediates[34, 47, 59]. It was first separated from the reaction system consisting of fructose, sucrose and oxalic acid, though not until 1909, the structure of HMF was assigned, because the extraction of HMF from aqueous phase was not easy due to the fact that the distribution coefficient between the inorganic and organic phase was not favorable[34, 60]. A similar method was provided by Kiermayer who produced HMF starting from sugar cane[61]. Later, HMF was found to be the product of carbohydrates dehydration reactions by Harris[62]. Up to now, over 1000 papers have been published on the synthesis of $\mathrm{HMF}$, which is an evidence for the great importance of HMF as a platform molecule. HMF is mainly synthesized by the dehydration of monosaccharides after loss of three water molecules. It is a very complex process because of the acid catalyzed dehydration leads to various by-products such as formic acid, 
levulinic acid apart from HMF. With the increasing interests in carbohydrates dehydration reactions, a series of catalysts have been developed for the synthesis of HMF. Generally, the catalysts can be classified into five groups: organic acids, inorganic acids, salts, Lewis acid, and others[63].

Sulfuric acid, phosphoric acid and hydrochloric acid are the most commonly used acid catalysts for the dehydration of carbohydrates to HMF[64-66]. Hydroiodic acid was also found to be active in the conversion of hexoses to HMF[34]. Organic acids such as oxalic acid, levulinic acid and p-toluenesulfonic acid can also act as catalysts[67-69]. $\mathrm{H}_{2} \mathrm{SO}_{4}$ worked as a catalyst in sub-critical water at $250^{\circ} \mathrm{C}$ and the yield of $\mathrm{HMF}$ was as high as 53\%[70]. $\mathrm{HCL}$ and $\mathrm{H}_{3} \mathrm{PO}_{4}$ can also catalyze the reaction in subcritical water to a HMF yield of 40-50\%. It was found that lower $\mathrm{pH}$ can lead to more rehydration of HMF to levulinic and formic acids. Among hydrochloric, sulfuric, phosphoric, oxalic, citric, maleic and p-toluenesulfonic acids, phosphoric acid showed best catalysis effect at lower $\mathrm{pH}$ while at higher $\mathrm{pH} \mathrm{HCl}$ was the best. Moreover, $\mathrm{HMF}$ can also be produced from glucose, but usually the reaction rate and selectivity are lower than that of fructose. For example, when $\mathrm{H}_{3} \mathrm{PO}_{4}$ was used as the catalyst for the dehydration of glucose, only $15.5 \%$ HMF yield can be obtained[71]. The ring structure of glucose is stable, thus there is little open-chain molecules in the reaction system, making the HMF generating speed limited by the low speed of enolization.

Lewis acid, such as $\mathrm{CrCl}_{2}, \mathrm{CrCl}_{3}, \mathrm{FeCl}_{2}, \mathrm{CuCl}_{2}, \mathrm{VCl}_{3}, \mathrm{MoCl}_{3}, \mathrm{PdCl}_{2}, \mathrm{PtCl}_{4}$, $\mathrm{RuCl}_{3}$ and $\mathrm{RhCl}_{3}$ are found to be effective in the catalytic dehydration of fructose to $\mathrm{HMF}$. $\mathrm{AlCl} 3$ was used as catalyst for fructose dehydration in water, and the yield of HMF is $53.9 \%$. 29.4\% HMF yield was obtained when inulin was used as the starting material under the same reaction conditions[72]. A combination of $\mathrm{FeCl}_{3}$ and tetrathyammonium bromide $\left(\mathrm{Et}_{4} \mathrm{NBr}\right)$ worked more effective than $\mathrm{FeCl}_{3}$ for the conversion of fructose to $\mathrm{HMF}$, in which the active species were thought to be $\mathrm{FeCl}_{3} \mathrm{Br}$ - ion, the yield of $\mathrm{HMF}$ can reach as high as $86 \%$ in 
$\mathrm{NMP}$ [73]. According to recent reports, $\mathrm{IrCl}_{3}$ and $\mathrm{GeCl}_{4}$ showed good catalytic activity for the dehydration of HMF in [BMIM]Cl, 89.2\% and 92.1\% HMF yields were obtained respectively under mild conditions[ $[74,75]$. In addition, rare earth metal trifluoromethanesulfonates exhibited high activities as new type of Lewis acid[76]. In the presence of DMSO, 83.8\% HMF yield was observed by using $\mathrm{Sc}(\mathrm{OTf}) 3$ as the catalyst. Under similar reaction conditions, HMF yields of $80.2 \%, 78.1 \%, 73 \%$ and $63.5 \%$ were achieved in the presence of $\mathrm{Yb}(\mathrm{OTf})_{3}$, $\mathrm{Ho}(\mathrm{OTf})_{3}, \mathrm{Hm}(\mathrm{OTf})_{3}$ and $\mathrm{Nd}(\mathrm{OTf})_{3}$ respectively.

Solid acid catalysts have several advantages over liquid acid catalysts. First, solid acid catalyst can be easily separated from the reaction system and can be reused. Second, solid acid catalyst can work at higher temperature by which the reaction can become shorter thus it can reduce the side reactions to a distant. Third, the acid density on the surface of solid acid can be adjusted since the acid groups are the active sites for the dehydration of fructose to HMF. A fructose conversion of $76 \%$ and a HMF selectivity of $92 \%$ were achieved by using dealuminated $\mathrm{H}$-form mordenite at $165^{\circ} \mathrm{C}$ in a solvent consisting of water and MIBK[77]. The conversion of fructose and selectivity of HMF are influenced by the kind of acid and the structural properties of the acid. When the $\mathrm{Si} / \mathrm{Al}$ ratio was $1: 1$, the H-mordenite showed best catalytic activity[78]. In the presence of the vanadylphosphate(VOP), a $40 \%$ yield of HMF was obtained for $6 \mathrm{wt} . \%$ aqueous solution of fructose[79]. Introducing different trivalent metal ions can enhance the VOP catalytic activity. The yield and selectivity increased to 50\% and $87 \%$ respectively using Fe-containing VOP catalyst. Nb-based catalysts like $\mathrm{Nb}_{2} \mathrm{O}_{5}$, niobium phosphate(NbOPO4) exhibited high catalytic acitivity[80, 81]. The $\mathrm{Nb}_{2} \mathrm{O}_{5}$ prepared at $400^{\circ} \mathrm{C}$ shows $100 \%$ fructose conversion with $86 \%$ yield of $\mathrm{HMF}$ in DMSO at $120 \mathrm{oC}$ after $2 \mathrm{~h}[35][82]$. Zr- and Ti-based catalyst with different structures were employed in the dehydration of fructose[83]. An optimized HMF yield of $47 \%$ was obtained within $4 \mathrm{~h}$ at $403 \mathrm{~K}$ over $\mathrm{SO}_{4}{ }^{2-} / \mathrm{ZrO}_{2-}$ 
$\mathrm{Al}_{2} \mathrm{O}_{3}$ with $\mathrm{Zr}-\mathrm{Al}$ mole ratio of $1: 1[84]$. The yield of $\mathrm{HMF}$ reached $38 \%$ catalyzed by $\mathrm{TiO} 2$ under microwave irradiation with $83 \%$ conversion of fructose at $200^{\circ} \mathrm{C}[85]$. In the same conditions, $30 \% \mathrm{HMF}$ yield and $65 \%$ fructose conversion can be obtained by $\mathrm{ZrO} 2$ instead of $\mathrm{TiO} 2[86]$. Cheap and readily abundant $\mathrm{Zr}(\mathrm{O}) \mathrm{Cl}_{2}$ catalyst produce $63 \% \mathrm{HMF}$ from fructose in water using methylisobutylketon(MIBK) as the organic phase in biphasiis solvent system[87]. Sulfated zirconia is an effective catalyst which can catalyzed fructose to HMF with the conversion of $94 \%$ and HMF yield of $72 \%$ [88]. $98 \%$ conversion of fructose with $85 \%$ HMF yield in $2 \mathrm{~h}$ at $120^{\circ} \mathrm{C}$ using $10 \mathrm{wt} . \%$ acidity modified silver exchanged silicontungstic acid catalyst was achieved. Ion-exchange resins also can be applied for the synthesis of HMF from sugars[89]. Nakamura developed a strongly acidic ion-exchange resin for the dehydration of fructose to HMF and obtained an $80 \%$ yield of HMF. An even higher HMF yield of $90 \%$ was obtained by the employment of Diaion ${ }^{\circ} \mathrm{R}$ PK-126 resin, which can be used in water-MIBK biphasic system by using DMSO or NMP as an aqueous-phase modifier[90]. Lansalot-Matras and Moreau used Amberlyst-15 as a catalyst in ionic liquids such 1-buty-3-methyl imidazolium tetrafluoroborate([BMIMBF4) and 1-butyl-3-methyl imidazolium hexafluorophosphate([BMIM]F6) for the dehydration of fructose to $\mathrm{HMF}[91]$. The reaction took place in a micro-batch reactor at $80^{\circ} \mathrm{C}$. The yield of $\mathrm{HMF}$ was around $50 \%$ and $80 \%$ respectively by

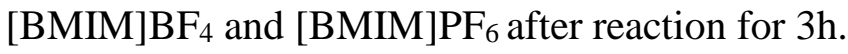

Researchers tried different solvents in the dehydration of glucose with mineral acid as catalyst. The selectivity of HMF was about $6 \%$ in water. Unlike fructose, even in an aprotic solvent such as DMSO and DMF, the yield of HMF was also very low[92]. Li Recently, a specially designed biphasic reactor which can help improve the yield of HMF from $11 \%$ to $53 \%$ was developed for the dehydration of glucose[93]. Compared with mineral acid catalysts, solid acid performed better in transferring glucose to HMF. 
Solvents plays an important role in the dehydration of carbohydrates to HMF[94]. DMSO, DMF, DMA, acetone, acetic acid, methanol, THF, MIBK and isopropanol were the most commonly used solvent for the dehydration of fructose to $\mathrm{HMF}$. When $\mathrm{HCl}$ was used as the catalyst for the dehydration of fructose to HMF, $70 \%$ and $83 \%$ yield of HMF were achieved in the presence of tert-butanol and isopropanol respectively[95]. The same thing happened when $\mathrm{H}_{2} \mathrm{SO}_{4}$ was used to catalyze the fructose dehydration reaction. 57\% yield of HMF was obtained in isopropanol at $120^{\circ} \mathrm{C}$ after $2 \mathrm{~h}$ [95], while $\mathrm{HMF}$ yield of $82.9 \%$ can be achieved at $100^{\circ} \mathrm{C}$ in $50 \mathrm{~min}$ by using [BMIM]Cl as the solvent[96]. Lewis acid catalyst such as $\mathrm{FeCl}_{3}$ combined with tetraethylammonium bromide exhibited different activities and selectivities towards fructose dehydration to HMF in different solvent. In the presence of NMP, the HMF yield can reach $86 \%$, while this value dropped to $62 \%$ when the reaction was conducted in DMF[73]. Formic acid and levulinic acid are the common byproducts formed during the reaction when organic solvents such as DMF, methanol and toluene are used as the solvents. However, when the reaction was carried out in DMSO, the formation of byproducts was inhibited and the HMF yield will increase. Musau and Munavu further studied the effect of DMSO and found that DMSO could facilitate the formation of HMF and prevent HMF from decomposing to formic acid and levulinic acid[97]. Similar findings were also reported when ionic liquids were applied in the dehydration of carbohydrates to HMF. Ionic liquids serve as both good solvents and catalyst during the reaction. Because of the special properties such as negligible vapor pressure and comparative thermal stability, there is more hope to develop more green approaches for the utilization of carbohydrates, thus ionic liquids are considered as more environmental friendly solvents than the other organic solvents. Molten salts were first used in the dehydration of monosaccharides in 1983[98]. 70\% yield of HMF converted from fructose was achieved in the presence of pyridinium chloride, which laid a solid foundation 
for the development of ionic liquids used in carbohydrates transformation. In recent years, 1-butyl-3-methylimidazolium bisulfate, N-methyl-2-pyrrolidonium methyl sulfonate, diethylene glycol-bis(3-methylimidazolium)dimesylate, triethylene glycol-bis(3-methylimidazolium) dimesylate and tetraethyleneglycol-bis(3-methylimidazolium) dimesylate were prepared and applied in the dehydration of fructose to HMF as catalysts and solvents[99-103]. Results showed that Lewis acidic ionic liquids were more effective than Bronsted acidic ionic liquids for the conversion of fructose to HMF.

There are two main reasons for the low HMF yield in some reaction systems, one is the rehydration of the formed HMF, the other is the formation of humins thought reaction between HMF and carbohydrates. To solve the above problems, biphasic solvent systems were developed to separate HMF from the reactant by extraction of HMF into organic phase. For example, mix solvents reactions were examined for the conversion of fructose to $\mathrm{HMF}$ using $\mathrm{HCl}$ as the catalyst. Under the same reaction conditions, HMF yields of $54.6 \%, 53.1 \%, 32 \%$ and $49.9 \%$ were observed in $\mathrm{H}_{2} \mathrm{O} / \mathrm{MIBK}, \mathrm{H}_{2} \mathrm{O} / 1$-butanol, $\mathrm{H} 2 \mathrm{O} / 1$-hexanol, and $\mathrm{H}_{2} \mathrm{O} /(2$-butanoltoluene) respectively[103-105]. HMF yield of 97\% was reported in a DMSO/MIBK biphasic system at $76^{\circ} \mathrm{C} . \mathrm{H}-\mathrm{Y}$ faujasites and $\mathrm{H}$-mordenite catalysts were examined in a $\mathrm{H} 2 \mathrm{O} / \mathrm{MIBK}$ system for the conversion of fructose to $\mathrm{HMF}[106,107]$. The ratio of $\mathrm{Si} / \mathrm{Al}$ affected the catalytic performance significantly. HMF yield can be achieved as high as $69 \%$ with $76 \%$ fructose conversion in the presence of $\mathrm{H}$-mordenite with $\mathrm{Si} / \mathrm{Al}$ ratio of 11. Dumesic et al. prepared propylsulfonic acid-functionalized and thiopropyl-modified silicas and applied in the dehydration of fructose to HMF in MIBK/2-butanol (7:3 w/w) at $180^{\circ} \mathrm{C}[108]$. Results suggested that the catalytic effect was enhanced only after the acid functionalization. Later, Dumesic et al also studied the effect of the ratios of water/DMSO and water/NMP mixtures on the dehydration of fructose to HMF in the presence of acidic ion-exchange resin DIAION PK216 at 90 and $120^{\circ} \mathrm{C}[93]$. 
The highest yield was $83 \%$ and fructose conversion was $98 \%$, with ratio of water/NMP as 4:6 (w/w). It was found that lower fructose concentration could benefit the selectivity of HMF. Yang et al. reported a very high yield of HMF and selectivity by using niobic acid treated with phosphoric acid[109]. The reaction was carried out in water and 1.5 equiv (v/v) 2-butanol at $160^{\circ} \mathrm{C}$, resulting in $89 \% \mathrm{HMF}$ yield and $90 \%$ fructose conversion. Fan et al. conducted the dehydration of fructose to $\mathrm{HMF}$ in water/MIBK in the presence of $\mathrm{Ag}_{3} \mathrm{PW}_{12} \mathrm{O}_{40}$ which is a solid heteropolyacid[110]. Under the optimized conditions, HMF yield of $78 \%$ and fructose conversion of $83 \%$ were obtained at $120^{\circ} \mathrm{C}$ after $1 \mathrm{~h}$. Analogously, $\mathrm{Cs}_{2.5} \mathrm{H}_{0.5} \mathrm{PW}_{12} \mathrm{O}_{40}$ was applied in the catalytic dehydration of fructose in water/MIBK, yielding $74 \% \mathrm{HMF}$ and $78 \%$ fructose conversion at $115^{\circ} \mathrm{C}$ after $1 \mathrm{~h}[111]$. HMF can also be produced with glucose as the precursor. $21-25 \%$ isolated yield of HMF was reported in water/MIBK after $9 \mathrm{~h}$ at $160^{\circ} \mathrm{C}$ from glucose[112]. Rigal and Gaset studied the aldose dehydration in water/MIBK with an acidic ion-exchange resin as the catalyst and HMF yields below $10 \%$ were obtained[113]. $\mathrm{HCl}$ was also used in the catalytic conversion of glucose to HMF, a HMF yield of $24 \%$ with glucose conversion of $50 \%$ was attained in $\mathrm{MIBK} / 2-$ butanol at $170^{\circ} \mathrm{C}[93]$. A relatively high $\mathrm{HMF}$ yield up to $62 \%$ was achieved by the combination of $\mathrm{AlCl}_{3}$ and $\mathrm{HCl}[114]$. Yang et al. reported a comparable result in a biphasic water $-\mathrm{NaCl} / \mathrm{THF}$ systems in the presence of $\mathrm{AlCl}_{3}$ [115]. Solvents systems such as $\mathrm{H}_{2} \mathrm{O}-\mathrm{DMSO} / 2-\mathrm{BuOH}-\mathrm{MIBK}$ were also investigated, giving the highest HMF yield of 35\%[116]. It can be seen that even in a biphasic system, conversion of glucose to HMF with high yield is more difficult than the conversion of fructose. Roman-Leshkov and Dumesic then investigated the influences of different types of organic solvents as extracting agents in combination with inorganic phase[117]. Typically, experiments were conducted with $30 \mathrm{wt} \%$ fructose in the aqueous phase with $\mathrm{HCl}$ as the catalyst at $150^{\circ} \mathrm{C}$, the volume ratio of the organic and inorganic solvent is 3.2. The study 
focused on the tests of primary and secondary alcohols, ketones and cyclic ethers. HMF yield of around $69 \%$ was the highest yield which can be achieved by using 2-butanone and 2-pentanol as the organic phase.

\subsection{Synthesis of 5-hydroxymethylfurfural-based furan derivatives}

Based on the further catalytic transformation of HMF, many furan derivatives such as DFF, HMFCA and FDA can be synthesized, that is the oxidation of HMF can be performed selectively to the formyl or hydroxyl groups or can involve both groups[118, 119].

The selective oxidation of the formyl group of HMF leads to the formation of HMFCA. Silver oxide and mixture of silver and copper were used as catalysts for the selectivity oxidation of the formyl group of HMF to HMFCA[120]. HMFCA was considered to be the intermediate product during the aerobic oxidation of $\mathrm{HMF}$ to FDA. $\mathrm{Au} / \mathrm{TiO} 2$ was applied in the oxidation reaction of $\mathrm{HMF}$, it was found that a lower pressure or low concentrations of base resulted in more of the intermediate oxidation product HMF than FDA[121]. Davis et al. reported that $92-93 \%$ selectivity towards HMFCA with $100 \%$ conversion of $\mathrm{HMF}$ were obtained in the presence of $\mathrm{Au} / \mathrm{C}$ in basic conditions[122]. Beside oxygen, $\mathrm{H}_{2} \mathrm{O}_{2}$ can be used as the oxidant also. As a minor product, HMFCA of $40 \%$ yield can be attained using chloroperoxidase as the catalyst[123].

The selectivity oxidation of hydroxyl group of HMF can produce DFF which is an important monomer for industry[124]. However, due to the high reactivity of the aldehyde function, the selective oxidation of HMF to DFF is still challenging. In early reports, DFF can be get from the oxidation of HMF by hydrogen peroxide and catalyzed by synthetic titanium silicalite, while the yield of DFF was only $25 \%[125]$. Various catalytic systems have been developed towards the selective oxidation of HMF to DFF. In early reports, classical oxidants such as pyridinium chlorochromate, $\mathrm{NaOCl}, 2,2,6,6$ tetramethylpiperidine-1-oxide (TEMPO) have been applied for the oxidation of 
HMF into DFF[118, 126, 127]. By using a DMSO-potassium dichromate oxidative complex at $100^{\circ} \mathrm{C}, 58 \%$ yield of DFF was obtained[128]. The trimethylammonium chlorochomate(TMACC)- $\mathrm{Al}_{2} \mathrm{O}_{3}$ and pyridinium chlorochromate( $\mathrm{PCC})-\mathrm{Al}_{2} \mathrm{O}_{3}$ oxidative system was tested and obtained $\mathrm{DFF}$ in $75 \%$ and $58 \%$ yield respectively[126, 128]. Homogeneous metal/bromide catalysts were also used in the aerobic oxidation of HMF to DFF, like $\mathrm{Co} / \mathrm{Mn} / \mathrm{Zr} / \mathrm{Br}$ was used with air as oxidant, it was found that $\mathrm{Co} / \mathrm{Mn} / \mathrm{Zr} / \mathrm{Br}$ was activity which mean that it can provide high conversion and selectivity, resulting in $57 \%$ DFF yield[36]. However, these methods showed some distinct drawbacks such as high cost and large amount of toxic waste. From the viewpoint of sustainable development, it is recognized that the oxidation process of HMF using molecular oxygen as the oxidant with heterogeneous catalysts is much more economical and environmentally friendly. The conversion of HMF to DFF with oxygen, air or other environmental friendly oxidants has long been a hot research topic. Supported $\mathrm{V}_{2} \mathrm{O}_{5} / \mathrm{TiO}_{2}$ catalyzed oxidation of $\mathrm{HMF}$ with air as the oxidant and toluene or MIBK as solvents was also investigated[129]. The yield of DFF can reach as high as $90 \%$ at $1.6 \mathrm{MPa}$ with in $4 \mathrm{~h}$. The influence of solvents, $\mathrm{pH}$ value, pressure of oxygen and temperature was investigated when $\mathrm{Pt} / \mathrm{C}$ catalyst was used in the reaction[130]. It was found that DFF was prone to form at high temperature and natural $\mathrm{pH}$.. Mental-modified unsupported or SiO2supported vanadyl phosphate(VOP) catalysts were also tested in the oxidation of HMF to DFF under oxygen and air pressure[131]. The influence of different solvents was investigated and researchers found that DMF was one of the solutions which performed best for this transformation. By using $\mathrm{NaClO}$ as oxidant and $\mathrm{Mn}(\mathrm{III})$-salen catalysts, HMF can be converted to DFF at room temperature without any by products like FDA[132]. $\mathrm{V}_{2} \mathrm{O}_{5}$ and $\mathrm{VOHPO}_{4} \cdot 0.5 \mathrm{H}_{2} \mathrm{O}$ were used for the oxidation of HMF to DFF. It was found that this kind of $\mathrm{V}$ catalyst can not only catalyzed pure HMF to DFF but also the DFF produced by 
dehydration of fructose[131]. Supported platinum catalysts were also applied in the conversion of HMF to DFF such as the $\mathrm{Pt} / \mathrm{SiO}_{2}$ and $\mathrm{Pt}-\mathrm{ZrO}$ for the oxidation of pure HMF[133].

FDA which is a potential bio-renewable replacement monomer for terephthalic acid in polyethylene terephthalate plastics and can be formed by the oxidation of both formyl and hydroxyl groups of HMF[134-136]. It was first reported to be synthesized by electrochemical oxidation of HMF in a H-shaped cell near an anode[137]. Strasser et la. Studied the electrochemical oxidation of HMF using a Pt electrode at pH 10[138]. Later, carbon-black-supported noblemetal catalysts were applied in the electrocatalytic oxidation of HMF and it was found that the reaction results were greatly affected by the potential and the electrocatalyst[139]. $\mathrm{N}_{2} \mathrm{O}_{4}$ and nitric acid can oxidize HMF to FDA in DMSO and obtained FDA in $24 \%$ yield[140]. $\mathrm{Pt} / \mathrm{Al}_{2} \mathrm{O}_{3}$ as a more economical and environmentally friendly heterogeneous metal catalyst was used under basic reaction conditions using air as oxidant at $60^{\circ} \mathrm{C}[133]$. When $\mathrm{Pt}-\mathrm{ZrO}_{2}$ and air were used in the synthesis of FDA from HMF, a 100\% conversion and 98\% selectivity were obtained. $\mathrm{Au} / \mathrm{TiO}_{2}$ nanoparticle catalyst catalyzed $\mathrm{HMF}$ to FDA in aq. $\mathrm{NaOH}$ at 20 bar $\mathrm{O}_{2}$ and ambient temperature lead to a $71 \%$ yield[122]. Through conducting the oxidation reaction with various gold nanoparticle catalysts, it was found that the rate-limiting step was the oxidation of HMFCA to FDA[139]. The most active and efficient catalysts were $\mathrm{Au}-\mathrm{CeO}_{2}$ and $\mathrm{Au}-\mathrm{TiO}_{2}$. According to the experiments conducted in the presence of supported platinum catalysts at different $\mathrm{pH}, \mathrm{Pt} / \mathrm{Al}_{2} \mathrm{O}_{3}$ and $\mathrm{Pt} / \mathrm{C}$ was found to be the most efficient catalysts in aqueous $\mathrm{Na}_{2} \mathrm{CO}_{3}$ while $\mathrm{Pt}-\mathrm{ZrO} 2$ provided the best oxidation results under acidic conditions. Compared to $\mathrm{Au} / \mathrm{C}$ and $\mathrm{Au} / \mathrm{TiO} 2, \mathrm{Pt} / \mathrm{C}$ and $\mathrm{Pd} / \mathrm{C}$ were more selective towards FDA under basic aqueous conditions. However, chemical oxidation reactions are usually conducted under high temperature and high pressure. Conversely, biocatalytic conversion is typically performed under 
relatively mind reaction conditions with less toxic chemicals. DFF was oxidized to FDA with high yield (>99\%) in the presence of lipases as biocatalysts and hydrogen peroxide as oxidant[141]. Most enzymes are restricted to either oxidation of formyl groups or hydroxyl groups, making the full oxidation of HMF to FDA very challenging. Fraaije et.al recently developed an FAD-dependnent oxidase of the glucose-methanol-choline oxidoreductase family for the HMF oxidation. Up to $95 \%$ yield of FDA was obtained at ambient pressure and temperature after $24 \mathrm{~h}[142,143]$.

Though various HMF-derived chemicals are very good starting materials for further transformations and chemical building blocks for industry, the industrial production of HMF-derived chemicals has not been realized because of the high costs using HMF as raw material. The high price of HMF is caused by the high energy cost and process complexity during the separation and purification of HMF from the reaction system. However, most exist catalysts are efficient only for the oxidation of pure HMF, it means that the catalysts show no activity for the in situ oxidation of HMF generated from carbohydrates. Thus it is necessary to develop new catalysts which are able to catalyze the one-pot synthesis of HMF-derived chemicals directly from carbohydrates in order to save energy and make the synthesis process greener. So far, vanadium based catalysts were found to be active for the air-oxidation of not only pure HMF but also HMF produced via dehydration of fructose. $\mathrm{V}_{2} \mathrm{O}_{5}$ was the least expensive and most readily available catalyst that was used for the one-pot transformation of fructose to DFF[144]. A 58\% yield of DFF calculated on HMF and $43 \%$ calculated on fructose were obtained by adding the $\mathrm{V}_{2} \mathrm{O}_{5}$ into the reaction system after the fructose was fully converted to $\mathrm{HMF}$. VOHPO ${ }_{4} \cdot \mathrm{H}_{2} \mathrm{O}$ was prepared and used in the one-pot reaction, exhibiting 61\% DFF yield calculated on HMF and 45\% calculated on fructose. Since the whole reaction consists two steps: the dehydration of carbohydrates to HMF and the oxidation of HMF to DFF, it is 
easy to imagine that the combination of acid sites and oxidative sites may lead to the formation of bifunctional catalysts which can catalyze both the dehydration reaction and oxidation reaction, resulting in a one-pot conversion of carbohydrates to DFF without the separation and purification of HMF. H-form cation and $\mathrm{VOHPO}_{4}$ were jointly used for the direct transformation of fructose to DFF in DMSO[145]. $\mathrm{CrCl}_{3} \cdot 6 \mathrm{H}_{2} \mathrm{O} / \mathrm{NaBr}$ and $\mathrm{NaVO}_{3} \cdot 2 \mathrm{H} 2 \mathrm{O}$ were applied in the one-pot synthesis of DFF from glucose in DMA with a DFF yield of 55\% based on glucose[146]. High yield of DFF was obtained from fructose by using the combination of $\mathrm{Fe}_{3} \mathrm{O}_{4}-\mathrm{SBA}-\mathrm{SO}_{3} \mathrm{H}$ and K-OMS-2. $\mathrm{Fe}_{3} \mathrm{O}_{4}-\mathrm{SBA}_{-} \mathrm{SO}_{3} \mathrm{H}$ can produce HMF from fructose with $81 \%$ yield in DMSO at $110^{\circ} \mathrm{C}$, while K-OMS2 can catalyze the oxidation of HMF with nearly $100 \%$ selectivity. However, when $\mathrm{Fe}_{3} \mathrm{O}_{4}-\mathrm{SBA}-\mathrm{SO}_{3} \mathrm{H}$ and $\mathrm{K}-\mathrm{OMS}-2$ were added together into the reaction system at the initial stage, the DFF yield became quite low due to the formation of humins or the unwanted oxidation of fructose by K-OMS-2[147]. Further study showed that K-OMS-2 did promote the decomposition of fructose to unknown compounds. Therefore, a one-pot but two-step reaction was conducted by stepwise addition of catalysts to overcome this problem. Similarly, a combination of HT, Amberlyst-15, and Ru/HT was found to be active for the production of DFF from fructose and glucose via base-catalyzed isomerization, acid-catalyzed dehydration and selective oxidation in a one-pot reaction[148]. The reaction was carried out under atmospheric pressure of molecular oxygen, DFF yield of $13 \%$ and HMF yield of $44 \%$ were obtained starting from fructose. DFF yield raised from $13 \%$ to $34 \%$ by a one-pot two-step reaction, during which the reaction reacted under $\mathrm{N}_{2}$ for $3 \mathrm{~h}$ before bubbling oxygen into the system. Furthermore, one-pot synthesis of DFF from glucose in the presence of Amberlyst-15, HT and Ru/HT was investigated. DFF yield of $25 \%$ was obtained by a two-step but one-pot reaction without separation of catalysts. While this value was only $8 \%$ by a one-step synthesis method due to the decomposition of 
glucose. For the above examples, DFF was produced through a one-pot but twostep reaction. Typically, the reaction was catalyzed by only acid catalyst first to convert fructose to HMF as much as possible, then the oxidative catalysts were added into the reaction system for the further oxidation of HMF generated from fructose to DFF. Another process is that acid catalysts and the oxidative catalysts were added together at the initial stage of the reaction, but the oxidant wasn't fed in until the fructose was totally dehydrated to HMF, inert gas was usually purged during the dehydration process to prevent the oxidation degradation of fructose. Recently, composite catalysts bearing both acid sites and oxidative sites were developed and applied for the one-step approach to DFF from fructose. As a solid acid, graphitic carbon nitride showed good performance in the dehydration of fructose to HMF, and vanadium-doped graphitic carbon nitride can act as the oxidative catalyst for the aerobic oxidation of HMF to DFF. Thus the combination of protonated graphitic carbon nitride and vanadium-doped graphitic carbon nitride can be applied in a one-pot and two-step synthesis method for the transformation of fructose to DFF. Since the acid sites and oxidative sites were supported on the same carrier, Jinzhu Chen et al. prepared a bifunctional catalyst made of protonated vanadium-doped graphitic carbon nitride and applied in the one-step approach to DFF rom fructose, leading to a DFF yield of 45\%[149]. Most recently, keggin-type heteropolyacids were found to be active in acid and oxidation catalysis for the transformation of hydrocarbons, hydrogenation of cellulose and hydrodexoygenation of lignin-derived monomers[150-152]. $\mathrm{H}_{3} \mathrm{PW}_{12} \mathrm{O}_{40}$ and $\mathrm{FePW}_{12} \mathrm{O}_{40}$ have been applied in the dehydration of fructose and degradation of cellulose[111]. They are well known for the well-defined structure, strong Bronsted acidity, fast multi-electron transfer, high proton mobility and resistane to hydrolytic and oxidative degradations in solution. Hu et al. synthesized several Keggin-type heteropoly acids such as $\mathrm{H}_{4} \mathrm{PMo}_{11} \mathrm{~V}_{1} \mathrm{O}_{40}, \quad \mathrm{H}_{5} \mathrm{PMo}_{10} \mathrm{~V}_{2} \mathrm{O}_{40}, \quad \mathrm{H}_{4} \mathrm{PW}_{11} \mathrm{~V}_{1} \mathrm{O}_{40}, \quad \mathrm{H}_{3} \mathrm{PMo}_{12} \mathrm{O}_{40} \quad$ and 
$\mathrm{H}_{5} \mathrm{SiMo}_{11} \mathrm{~V}_{1} \mathrm{O}_{40}$ and studied their catalytic performance for the conversion of fructose to DFF[153]. Effects of the amount of catalyst, the redox potential and acidity were investigated and results showed that higher Bronsted acidity of heteropoly acids lead to higher yield of HMF from fructose and higher redox potential favors the oxidation of HMF to DFF. Under optimized conditions, DFF yield of $60.3 \%$ can be obtained at $160^{\circ} \mathrm{C}$ in $2 \mathrm{~h}$ in the presence of $\mathrm{PMo}_{12} \mathrm{HPA}$.. Considering the reusability of catalysts, homogeneous PMo12 HPA was converted to heterogonous catalyst by partial substitution of $\mathrm{H}^{+}$for $\mathrm{Cs}^{+}$. A series of $\mathrm{CsxH}_{3}-\mathrm{xPMo}_{12}$ catalysts were prepared and used in the one-step transformation of fructose to DFF. An impressive DFF yield of $69.3 \%$ was attained over $\mathrm{Cs}_{0.5} \mathrm{H}_{2.5} \mathrm{PMo}_{12}$ under optimized reaction conditions. Analogously, a series of acidic cesium salts of molybdovanadophosphoric heteropolyacids were developed by Chen et al[154]. as bifunctional catalysts for one-step approach to DFF from fructose with molecular oxygen as the oxidant under mild and environmental friendly conditions. The acid density and vanadium content are the two key factors influencing the catalytic performance. A maximum yield of $60 \%$ from fructose was achieved with $\mathrm{Cs}_{3} \mathrm{HPMo}_{11} \mathrm{VO}_{40}$ as a catalyst under atmospheric pressure of oxygen. Moreover, the heterogeneous $\mathrm{Cs}_{3} \mathrm{HPMo}_{11} \mathrm{VO}_{40}$ showed very good reusability during the recycling test. K-10 clay-Mo, a heterogeneous catalyst was successfully prepared by immobilization of bis(acetylacetonato) dioxo-molybdenum on montmorillonite K-10 clay[155]. In addition, a magnetic bifunctional $\mathrm{WO}_{3} \mathrm{HO}-\mathrm{VO}-\mathrm{SIO}_{2} @ \mathrm{Fe}_{3} \mathrm{O}_{4}$ nanocatalyst was prepared by using chlorosilylated $\mathrm{SiO}_{2} @ \mathrm{Fe}_{3} \mathrm{O}_{4}$ nanoparticles as the platform for the acidic and oxidative function groups[156]. The catalyst showed good performance in the fructose dehydration reaction with $82 \%$ HMF yield in $1 \mathrm{~h}$, and upon co-oxidant $\mathrm{H}_{2} \mathrm{O}_{2}$ DFF yield of $71 \%$ was achieved addition after $15 \mathrm{~h}$ under ambient air. It can be inferred that metal oxides represented by vanadium oxide and molybdenum oxide usually act as the oxidative sites to work along with the 
acid sites in order to make the catalysts active both for the dehydration of fructose and the oxidation of HMF to DFF. Most recently, graphene oxide served as a bifunctional and metal-free catalyst was applied in the direct syntheisis of DFF from fructose[157]. Under optimized conditions, 93.3\% yield of HMF from fructose dehydration was obtained and 90\% yield of DFF was achieved rom aerobic oxidation of HMF. Besides, the GO can catalyze the one-pot and onestep transformation of fructose to DFF in a yield of 53\%, and this value can increase to $72.5 \%$ when the reaction was conducted in two-steps, that is, to use nitrogen as the protection gas during the dehydration of fructose to HMF to prevent the oxidative degradation of fructose, and oxygen was fed in after the fructose has been converted to HMF.

\subsection{Synthesis and applications of carbon-based acid catalysts}

With efficient activity, high selectivity, long catalytic life and easy in separation and reuse, heterogeneous acid catalysts have attracted much attention for its potential in efficiently transforming biomass materials to value added chemicals[158]. There are five groups of solid acid catalysts which has long been used in the process of utilization of biomass materials. They are H-form zeolites, transition-metal oxides, cation-exchange resins, supported solid acids and heteropoly compounds[159].

Being non-toxic and no-corrosive, various zeolites are used in the transformation of biomass as good reusable catalysts[160, 161]. The acid sites are usually the active sites for the catalytic process, thus the acid density is a key factor for the performance of the catalysts[162]. The ratio of AllSi influences the Bronsted acid sites significantly for H-form zeolites[163]. Modified H-zeolites showed good stability, which can keep the framework at temperatures up to $127^{\circ} \mathrm{C}[161]$. Moreover, the physical properties such as hydrophilic or hydrophobic surfaces of a zeolite can be adjusted without influence of the functionalized acid sites. 
Metal oxide can have both Lewis acid sites and anions with Bronsted base sites on its surface[164]. Transition-metal oxides such as $\mathrm{Nb}_{2} \mathrm{O}_{5}, \mathrm{WO}_{3}, \mathrm{Zr}$-TMS, $\mathrm{TiO}_{2}$ and $\mathrm{Ta}_{2} \mathrm{O}_{5}-\mathrm{WO}_{3}$ are usually used as solid acid catalysts for biomass hydrolysis[165-168]. They are easy to reuse and have good stability during the reactions and can be classified into two types: single metal oxides and mix metal oxides. High surface area, adjustable pore size and good thermal stability are usually the special structure of mesoporous metal oxides which showed high catalytic efficiency[169-173].

Cation-exchange resins are widely used in esterification, alkylation, hydration, dehydration and condensation reactions as solid acids[174-178]. Acidic resins were used in the hydrolysis of cellulose since 1960[179]. Since then, cation-exchange resins such as NKC-9, Amberlyst-15 and Nafion NR50 are developed and applied in cellulose hydrolysis to glucose and showed considerable activity[180, 181]. Cation-exchange resins have many favorable properties and of these properties, most notably is the unique merit that inhibitors in the hydrolyzates can be removed by the resins themselves[182].

Having strong Bronsted acidity, stability and high proton mobility, heteropolyacids are used as environmentally benign and economical solid catalysts in homogeneous, biphasic and heterogeneous systems. Keggin heteropoly anion is the first characterized heteropoly acid. Heteropolyacids are soluble in water and their acidic strength is comparable with sulfuric acid. Moreover, they can be heterogenized through ion-exchange of $\mathrm{H}^{+}$with $\mathrm{Cs}^{+}$to form insoluble acidic cesium salts, $\mathrm{CsxH}_{3-\mathrm{x}} \mathrm{PW}_{12} \mathrm{O}_{40}[183,184]$. Other ions shuch as $\mathrm{V}^{5+}, \mathrm{Co}^{2+}$ and $\mathrm{Zn}^{2+}$ can substitute the metal ions of the heteropoly acids during the synthesis process[185]. In addition, heteropoly acids can be loaded onto functional carriers like amorphosilica, molecular sieve MCM-41 and activatedcarbon. The supported heteropoly acids can keep the activity and have better stability in liquid-phase reaction systems[186-193]. 
Compared with zeolites and transition-metal oxides, supported solid acid catalysts are promising for the utilization of biomass materials due to the substantial surface acidic species[194-196]. Supported metal oxide solid acid and supported carbonaceous solid acid are the two main types of supported solid acids. Typically, supported metal oxide solid acid catalysts are prepared by impregnating the hydroxide from ammonia precipitation of corresponding metal salt solution with sulfuric acid solution followed by calcination[197, 198]. The advantages of this kind of catalysts are their excellent thermal and mechanical stability, high surface area and large pore size. The disadvantage is that leaching of the acidic sites exist under hydrolytic conditions[199].

Among the various types of solid acid catalysts, carbon based catalysts attracted the most attention, because researchers are trying to prepare catalysts from renewable sources such as biomass material in order to make the chemical production process fully environmentally friendly and green. Compared with other materials, carbon material is considered as a very promising material due to its excellent properties such as low material cost, high surface area and good thermal stability[200]. They are good support materials and can be functionalized with acids or bases on the carbon surface. generally, there are two methods to prepare carbon-based acid catalysts, one is direct sulfonation of carbon material, the other is sulfonation of carbon material via reductive alkylation/acylation[180, 201, 202]. Carbon materials such as sugars, polycyclic aromatic compounds, polystyrene resins, activated carbon, biochar and lignin can act as the carbon sources during the sulfonation process[203]. Sulfonation agent, sulfonation time and carbon precursors are the main factors which influence the physical and chemical properties of the carbon-based acid catalysts. Various low polycyclic aromatic hydrocarbons such as naphthaline, anthracene were used as raw materials and directly applied in the incomplete sulfuric acid carbonization to prepare carbon-based solid acid, which showed high activities in esterification, 
hydrolysis reactions[204, 205]. But the sulfuric groups on the carbon supports is not stable, resulting in leaching of functional groups during the reaction at somewhat high temperature. It is of great significance to prepare carbon-based solid acid with biomass materials as staring materials to reduce the cost. Pyrolysis, gasification, flash carbonization and hydrothermal carbonization are the most commonly used methods for the carbonization of biomass or biomass derived materials[204].

In a typical direct sulfonation process, carbohydrates such as glucose, fructose, sucrose, and cellulose are heated under nitrogen to produce a browblack solid. Then the solid was sulfonated by concentrated sulfuric acid or fuming sulfuric acid at $150^{\circ} \mathrm{C}$. The carbon-based solid acid is collected after washing repeatedly with hot distilled water until no impurities such as sulfate ions were detected in the wash water[201]. Lower pyrolysis temperatures with longer time were found to be more suitable for the thermal carbonization of biomass materials to produce carbon materials which were more prone to be sulfonated to a high degree. For the hydrothermal carbonization method, the needed reaction temperature is much lower than pyrolysis, and the produced biochar has more surface oxygen containing groups.

Concentrated and fuming sulfuric acid are usually used as the sulfonation agents, and the latter is favorable for the synthesis of carbon-based solid acid with higher acid density and higher catalytic activity. For example, sulfonated-acitve carbon catalysts were tested in the esterification of higher fatty acids in ethanololeic acid mixture and ethanol-stearic acid mixture and the results showed that sulfonated-active carbon prepared with fuming sulfuric acid showed higher acid density and better catalytic performance. However, considering the safety and operation problems brought by fuming sulfuric acid, the application of concentrated sulfuric acid is more advocated. But when carbon nanotubes, graphene, and ordered mesoporous carbon are used as the carbon supports, it is 
difficult to sulfonate these materials by concentrated or fuming sulfuric acid. Special sulfonating agents such as 4-benzenediazoniumsulfonate, pstyrenesulfonic acid and Chlorosulfuric acid are used for the sulfonation of these "rigid" carbon materials[206-212]. The advantage of using these sulfonating agents is that the sulfonation temperature is very low, which can help maintain the original structure of the carbon materials. Recently, p-toluenesulfonic acid and hydroxyethylfufonic acid were also used in the sulfonation process. However, they can only be used with some special carbon materials which are mainly hydroxyl compounds and aldehydes so that they can polymerized together to form carbon-based acid in hydrothermal conditions[213-215]. For instance, glucose/resorcinol/TsOH mixtures are used as raw materials and polymerized to monolithic carbons with sulfonic groups. Moreover, glucose/ceric acid/hydroxyethylsulfonic acid mixtures can be used to prepare carbon-based solid acid through hydrothermal method. The effect of carbon precursor on the activity of such catalysts was studied by using different carbohydrates such as glucose, fructose, cellulose, sucrose and starch, and results showed that starch was the best starting material for the hydrothermal preparation of carbon-based solid acid due to high acid density, large surface area, pore volume and pore size[216, 217].

Carbon-based solid acid catalysts with special polymeric structure can be prepared by using resin as the carbon source[218-220]. Phenolic resin was used as raw material to synthesize carbon-based solid acid through two steps: carbonization and sulfonation[221]. The final products owed higher recyclability and higher $-\mathrm{SO}_{3} \mathrm{H}$ than the carbon-based solid acid prepared from traditional nafion resin. Similarly, mesoporous carbon-based solid acid can be prepared from resorcinol-formaldehyde resin and showed excellent catalytic performance for the selective dimerization of a-methylstyrene. In addition, polyvinyl chloride 
was as used to prepare carbon-based solid acid which performed better on the diffusion of reactants[222].

Carbon-based solid acid catalyst bearing $-\mathrm{SO}_{3} \mathrm{H}$, $-\mathrm{COOH}$, and $-\mathrm{OH}$ function groups, were efficient catalysts for the hydrolysis of cellulose[222-228]. The catalytic effect depends greatly on the ability of adsorbing $\beta-1,4$ glucan, the large effective area in water and acid groups tolerable to hydration[222]. A remarkable glucose yield of $40 \%$ with a high selectivity of $90 \%$ was obtained for the cellulose hydrolysis reaction in the presence of carbon-based solid acid[225]. Moreover, the carbon-based solid acid catalysts were found to be active for hydrolysis of hemicellulose to xylose and glucose, the dehydration from xylose to furfural and dehydration of fructose to furfural and HMF, which are important reactions in the utilization of biomass resources[229-232]. Carbon-based solid acid catalysts can be used in the production of biodiesel. It was found that the carbon-based solid acid with higher surface area and higher acid density usually showed better reaction performance[233]. Glycerol etherification with isobutylene can also be catalyzed by carbon-based solid acid catalysts with high selectivity[234]. Vegetable oil asphalt and s-MWCNTs were used as carbon precursors to prepare carbon-based solid acid catalysts. Though the asphalt-based catalyst had a lower surface of $7.48 \mathrm{~m}^{2} / \mathrm{g}$ compared to the higher surface area of $43.9 \mathrm{~m}^{2} / \mathrm{g}$ of s-MWCNTs-based solid acid, it showed higher activity for the production of biodiesel because of its high acid site density and large pores presented in the structure which allowed the diffusion of reactant easily into the internal acid groups[235, 236]. This result is consistent with the work presented by Lou et al. in which the catalytic activity of as-synthesized catalysts was primarily affected by acid sites densities but not the surface area[237]. De-oiled canola meal was used as carbon precursor by Similarly Rao et al. to prepare carbon-based solid acids in four different ways: direct sulfuric acid sulfonation, partial carbonization of de-oiled canola metal at $400^{\circ} \mathrm{C}$ followed by sulfuric acid 
treatment, partial carbonization of de-oiled canola metal at $400^{\circ} \mathrm{C}$ followed by steam activation and sulfuric acid sulfonation and partial carbonation of deo-iled canola metal at $300^{\circ} \mathrm{C}$ followed by sulfuric acid treatment. Results showed that the acid densities were greatly affected by the carbonation temperature. The acid sites density of the carbon-based solid catalysts prepared by direct sulfonation of de-oiled canola metal was the lowest compared with the other catalysts[238]. This is probably because of that the direct sulfonation destroyed the main components such as carbohydrates and proteins of de-oiled canola metal, while the formation of functional groups such as hydroxyl and aromatic structures during the carbonation process can react with sulfuric acid to form $-\mathrm{SO}^{3} \mathrm{H}$ groups The catalyst prepared by sulfonating de-oiled canola metal carbonized at $300^{\circ} \mathrm{C}$ showed the best activity for the production of biodiesel due to the highest acid sites density and largest pores. The partial carbonization of de-oiled canola metal at $300 \mathrm{oC}$ retained more functional groups which can react with sulfuric acid to produce more acidic functionalities on the surface. Under optimization conditions, the maximum conversion up to $93.8 \%$ of waste oil was obtained in the presence of such catalysts.

Carbon-based solid acid catalysts were also used in Michael-type FriedelCrafts reactions of indoles with a,b-unsaturated carbonyl compounds in water[239]. 85-96\% yield of isolated product without by-products was achieved by using of 5\% carbon-based solid acid in the reaction of indole and its derivatives with methyl vinyl ketone at ambient temperature. In the presence of carbon nanotube-based solid acid catalyst, 2-tert-butylhyroquinone, a widely used antioxidant, can be prepared by alkylation of hydroquinone with tert-butanol in a yield of $53.7 \%$ and conversion of $73.3 \%$ [240].

Carbon-based solid acid shows its advantages such as shorter reaction times, higher yield and simple workup over the aldol condensation reaction which is an important carbon-carbon bond-forming process in organic synthesis. Moreover, 
carbon-based solid acid catalysts showed higher catalytic activity than the traditional solid acid catalysts such as zeolite and Amberlyst-15 in the oxathioketalization reaction[215, 241, 242]. Carbon-based solid acid catalysts prepared from $\mathrm{C} / \mathrm{Si}$ composite showed high catalytic ability in the dimerization of methylstyrene[233, 243].

Carbon-based solid acid can also be applied in some applications such as the separation of special compounds, oxidation and electrochemical reactions. Sulfonated carbon-nanotube was used as an effective electrochemical catalyst for the oxidation of ethanol and ethylene glycol in alkaline medium[244, 245]. Carbon-based solid acid catalysts showed remarkable catalytic performance for the tetrahydropyranylation of alcohols and phenols in dichloromethane[246]. Moreover, the production of corresponding alcohols from 3,4-Dihydro-2H-pyran ethers can also be catalyzed by carbon-based solid acid in the presence of methanol.

\subsection{Synthesis and applications of carbon-supported metal catalysts}

Carbon materials such as active carbon, graphite, carbon black, carbon nanofibers/nanotubes and so on are widely used as supports to load the active sites i.e. metal, metal oxide and metal sulfide to stabilize the active phase and prepare carbon-supported metal catalyst. Because of the low cost and mass availability, active carbon and carbon black with high surface area are the most common carbon materials to be used for the preparation of carbon-supported catalysts. Prepared by pyrolysis of carbon precursor such as organic polymers and hydrocarbon at high temperature, carbon black is an amorphous carbon which is composed with roughly planar layers of sp2 hybridized carbons[247]. Active carbon is also amorphous carbon with large surface area and pore volume, preparing by high temperature pyrolysis of carbon materials followed by physical or chemical activation. Along with the development and progress of carbon science and technology, people has synthesized various carbon materials such as 
carbon nanoparticles, carbon nanotubes, graphene and mesoporous carbons. Carbon nanoparticles are usually prepared by the hydrothermal carbonization of biomass[248]. Carbon nanotubes and multiwall carbon nanotubes are the typical one-dimensional carbon materials, serving as ideal and unique templates for immobilization of metallic nanoparticles[249]. As a two-dimensional carbon material, graphene has remarkable surface area and unique physicochemical properties. It can be functionalized with metallic nanoparticles, polymers, doped with nitrogen and conjugated with various kinds of functional groups[250-256]. Three-dimensional carbon porous materials are usually prepared through template methods or sol-gel polycondensation of organic monomers[257-259].

Carbon materials are popular choice as support materials due to their remarkable physical and chemical properties such as mechanical strength, pore distribution, chemical and thermal stability. Wet-impregnation, incipientwetness impregnation and ion adsorption are the most common methods to prepare carbon-supported metal catalysts. carbon support materials are put into large amount of the metal precursors solution, then the solvent was removed by evaporation, the final carbon supported metal catalysts are collected after optional calcination and reduction. During the evaporation process, the metal precursor in the solution out of the pores of the carbon support will deposit on the outer surface of the support, forming large particles. To overcome this disadvantages, incipient-wetness impregnation method was developed by using just enough amount of metal precursor solution to fill the pores of the carbon support to prevent the depositing of the metal precursor on the outer surface of the support. If the metal precursor ions can be adsorbed on the surface of the carbon support, the support material can be filtered out and dried, calcined and reduced to produce carbon-supported metal catalyst. This method can be called ion adsorption. The coulombic interaction between the support and the metal precursors is the factor which influences the distribution of the precursor over the 
support. Generally, the negatively charged surface absorbs positively charged precursors and the positively charged surface attracts negatively charged precursors. Thus the surface properties of the carbon support play an important role in the formation of the carbon-supported metal catalysts[260]. During the preparation of carbon-supported Pt catalyst reported by Prado-Burguete, when heat-treated carbon black was used as the support, the Pt particle size was $5.1 \mathrm{~nm}$, this size can decrease to $2.4 \mathrm{~nm}$ when the carbon black was treated with $\mathrm{H}_{2} \mathrm{O}_{2}$ to introduce oxygen groups and then used as the support[261].

The same phenomenon occurred when active carbon and carbon black were used to prepare carbon-supported Pd catalyst[262]. Results showed that the metal dispersion decrease with increasing content of surface oxygen groups. Zhu et al also reported the similar findings. $5.7 \mathrm{~nm} \mathrm{Ru}$ particles were formed from $\mathrm{RuCl}_{3}$ using untreated active carbon, while $51 \mathrm{~nm}$ particles could be obtained under the same synthesis conditions by using heat-treated active carbon as the support[263, 264]. What's more, reoxidation of the heat-treated carbon material resulted under the same synthesis conditions in $3.8 \mathrm{~nm}$ particles. Furthermore, when the heatedtreated carbon material was subsequently treated in $\mathrm{CO}_{2}$ followed by nitric acid and then used as support, the smallest particles with size of $2.2 \mathrm{~nm}$ were obtained. The size difference of the particles can be explained by the changes of the surface groups that the $\mathrm{CO}_{2}$ treatment improved the mesoporous structure and the nitric acid treatment reduced the ash content which is beneficial for dispersion. Generally, the exist of oxygen groups on the surface of the carbon supports is conducive to the formation of small particles. But sometimes the results seem do not follow this principle completely, one of the reasons is that the amount of the oxygen groups such as carboxylic acid groups, might change during the impregnation procedure[265]. A typical example is that under a certain $\mathrm{pH}$ value, the carboxylic anhydride groups which can't absorb metal ions can hydrolyze to carboxylic groups which is available for adsorption. Gurrah et al., Van Dam and 
Van Bekkum and Sepulevda-Escribano et al. further studied the adsorption of the metal precursors and the oxygen containing groups on the carbon supports and found that high dispersion was formed during the decomposition step but agglomeration occurred due to the decomposition of oxygen groups under hightemperature treatment[266-268]. It was reported by Fraga et al. that increasing amount of oxygen groups on the support could lead to higher metal loading[269]. This is because that water is the most commonly used solvent, the oxygen funcitionalities can enhance the wettability of the carbon support. In addition, the exist of other ions in the solution also affect the loading amount of the metal precursors[270, 271].

Another factor which influences the dispersion of the active phase is the pore structure of the carbon support. Two activated carbons with similar oxygen amount and $\mathrm{pKa}$ but different pore volumes are used as carbon supports to prepare carbon support Pt catalysts, resulting in $1 \mathrm{wt} \%$ loading amount with a dispersion of $35 \%$ on the high-pore-volume support and $0.7 \mathrm{wt} \%$ metal loading and a dispersion of 20 irrespective of the precursor[272]. Samant et al. claimed that Pt particles of 1-2 $\mathrm{nm}$ were successfully loaded on a highly mesoporous carbon with a surface area of $534 \mathrm{~m}^{2} / \mathrm{g}$ [273]. similarly, Ru particles of $2.2 \mathrm{~nm}$ were obtained using high-temperature $\mathrm{CO}_{2}$ treated and $\mathrm{HNO}_{3}$ oxidized mesoporous carbon as the support[273]. These work demonstrated that the high mesoporous area was a key factor to prepare highly dispersed and highly loaded carbon-supported metal catalyst.

Despite drying and adsorption, the deposition of metal precursors from the solution onto supports can also be done by chemical reactions, which is called deposition precipitation. Through increase of $\mathrm{pH}$, change of valence of the metal ion and removal of a stabilizing ligand of the metal ion, the metal precursors can be deposited on the carbon support. Deposition precipitation method has been widely used to prepare carbon-supported metal catalyst due to its advantages such 
as reproducibility, high metal dispersions at high metal loadings. The strong interaction between the metal hydroxide and the support is essential for the metal precursors to decompose on the surface of the support rather than in the bulk liquid phase[274, 275].

For most solution of metal precursors, the deposition starts when the $\mathrm{pH}$ of the solution increase to a certain value. Urea is widely used for the decomposition of metal hydroxide on the carbon support, in which the reactant was added at low temperature and the hydrolysis of urea takes place uniformly. It was claimed by Van Der Lee et al. that the carboxylic acid groups on the carbon support surface are the important nucleation sites for the deposition of nickel hydroxide[276]. Moreover, the change of metal valence can also promote the precipitation. The meal decomposition process can be controlled by the concentrations, temperature, and the support.[277-280] What is interesting is that bimetallic catalysts can be prepared using this reduction deposition precipitation method through the catalyzed redox reaction of the first metal to the second metal[281]. In some cases, carbon support can act as a reducing agent to reduce the metal precursors in the salt solution and facilitate the decomposition without external reductant[282, 283]. Highly oriented pyrolytic graphite can be used to reduce Pt and result in carbon-supported Pt catalyst. In addition, removal of the ligands such as ammonia and EDTA which are widely used to stabilized transition metal ions in water via evaporation or oxidation can trigger the deposition of metal precursors onto the carbon support[284, 285]. Bezemer et al. prepared highly loaded and highly dispersed $\mathrm{Co} / \mathrm{CNF}$ through deposition of cobalt onto alumina starting from a cobalt amine complex by removing the ammonia slowly from the solution[286].

In order to further increase the loading amount of metal, researchers have developed some new methods. Different reductants were applied in the synthesis process and showed different effect on the control of the dispersions. For example, when formaldehyde was used to reduce Pt for the preparation of Pt/CNT, the 
produced Pt particles have a size of $3.4 \mathrm{~nm}$ with $10 \%$ loading amount, while when Pt was reduced using ethylene glycol, the resulted Pt particles had a size of $2.6 \mathrm{~nm}$ with the same loading amount[287]. It was also found that the particle size increased with the increasing of the water amount in the solution. Chen et al. synthesized $20 \mathrm{wt} \%$ of spherical Pt particles by performing the drying step by microwave heating[288]. Yang et al. claimed that ultrasonic treatment can help in preparation of $\mathrm{Pt}-\mathrm{Ru}$ particles with high level of metal dispersion on carbon black[289].

Carbon-supported catalysts are widely used in the catalytic transformation of biomass materials to value added chemicals. Carbon-supported Ru catalyst was applied in the hydrolysis of cellulose to glucose and hydrogenates glucose to sugar alcohols[290]. Tungsten carbide nanopowder supported on a treedimensional mesoporous carbon enhances the ethylene glycol yield to $72.9 \%$ through direct conversion of cellulose[291]. Similarly, 80\% yield of hexitol was obtained in the presence of a sea-urchin-like three-dimensional mesoporous carbon for the hydrolytic hydrogenation of cellulose[291]. Furfural yield of 67$69 \%$ and xylose conversion of $95-97 \%$ were obtained by graphene oxide or carbon black supported titanium dioxide nanoparticles catalyzing aqueous phase dehydration of xylose into furfural at $170^{\circ} \mathrm{C}[292]$. Pt/RGO prepared by microwave-assisted reduction method was employed on the conversion of cellulose to sorbitol, resulting in sorbitol yield up to $58.9 \%[293]$. Pt/C, Ru/C and Raney Ni catalysts were used in the hydrogenationo of xylose to xylitol, among which Pt nanoparticles supported on MWCNTs showed higher activity than the other commercial catalysts with $100 \%$ xylose conversion and $99.3 \%$ selectivity to xylitol[294, 295]. Ru/C are used for the conversion of glucose to sugar alcohols by a two-step hydrolysis and hydrogenation reaction[290]. Carbohydrates can be converted to unsaturated compounds though hydrodexygenation in the presence of carbon-supported metal catalysts. $\mathrm{Mo}_{2} \mathrm{C}$ is 
an active catalyst for the hydrodeoxygenation of vegetable oil[296-299]. Tungsten carbide can catalyze the hydrodeoxygenation of propanal and propanol to propene[300]. Results showed that smaller particle size of the tungsten carbide favors the improvement of the reaction activity. Hydrodeoxygenation reactions catalyzed by carbon-supported metal are also needed in the upgradation of biooils which are derived from the pyrolysis of woody biomass. As a by-product of biodiesel production, glycerol can be transformed to value-added chemicals in the presences of carbon-supported catalysts such as $\mathrm{Ru}$ and $\mathrm{Pt}$ particles on activated carbon. $\mathrm{Ru} / \mathrm{C}$ is more active than $\mathrm{Pt} / \mathrm{C}$ at neutral $\mathrm{pH}$ for the conversion of glycerol to glycols, while $\mathrm{Pt} / \mathrm{C}$ is more suitable for the formation of propylene glycol. Carbon-supported metal catalysts can also be used in the production of lactic acid which is usually produced through the biosynthesis by bacteria with glucose and sucrose as the substrates. Cu-graphene catalysts are applied in the conversion of glycerol, xylitol and sorbitol to lactic acid and showed good catalytic activity. $\mathrm{Ru} / \mathrm{C}$ was found to be the most active catalyst for the hydrogenolysis of biomass-reived xylitol to ethylene glycol and propylene glycol[301]. Pt/C and Ni/C are also used in the aqueous phase reformation of xylitol[302, 303]. Carbon-supported noble metal catalysts are widely used in the selective oxidation of HMF which is an important intermediate in the utilization of carbohydrates[304-306].

\subsection{Synthesis and applications of carbon sphere catalysts}

Carbon materials have been the active research fields for a long time. Many different shape carbon materials have been synthesized and attracted a great deal of attention because of their excellent physical and chemical properties. The shaped carbon materials include tubes, fibers, spheres, horns, calabashes and flasks etc.[307-315]. In this chapter we mainly focus on the carbon materials with spherical shape. Carbon balls, carbon spheres, carbon onions and mesoporous microbeads are the names given to this type of spherically shaped carbon. 
Carbon spheres are known to have a wide range of diameters from several nanometers to many micrometers. This will hold for the surface areas of the carbon spheres. The surface areas of carbon spheres vary from less than $1 \mathrm{~m}^{2} / \mathrm{g}$ to around $1000 \mathrm{~m}^{2} / \mathrm{g}[316]$. The carbon chains/layers/flakes staggerly weaved together to form the carbon sphere with different chemical and physical properties. Moreover, hollow carbon spheres can be synthesized by various strategies. The most common method is the templating process which coat the carbon materials on spherical template material such as metal complex, silica sphere followed by the removal of the core[317, 318].

According to the reaction temperature, the synthesis methods of carbon spheres can be divided into two types. The first one is based on the decomposition of carbon containing materials under high temperature in inert atmosphere. The second method usually consist of low temperature pyrolysis and catalytic decomposition of organic compounds by heat treatment of polymers or other materials in autoclaves. The synthesis conditions such as carbon sources, oxygen, catalysts, temperature and pressure influence the properties of the prepared carbon spheres significantly.

Arc-discharge process which was used to prepare carbon nanotube can also produce carbon spheres. He et al claimed the synthesis of carbon spheres though DC arc-discharge method by using acetylene and coke powder as carbon source[319]. Polyethylene terephthalate resin was used for the synthesis of carbon spheres at $1100^{\circ} \mathrm{C}$ through non-classical arc-discharge method[320]. Thermal plasma treatment is another method which can be used for the synthesis of both carbon tubes and carbon spheres. During the process, the carbon precursor was vaporized in the high temperature reactor and then carbon particles formed after the condense of the vaporized carbon precursors[321]. Hollow carbon spheres were synthesized by Yang et al. using methane/hydrogen as the carbon source and silica supported $\mathrm{MgO}$ and $\mathrm{Ni}$ nanoparticles as the catalyst 
through radio frequency plasma enhanced CVD[322]. Carbon spheres were also reported to be produced by use of a high pressure shock compression technique. Chemical vapor deposition which is an important method for the synthesis of shaped carbon materials, is a process in which carbon materials are developed from volatile carbon precursors. Toluene was reported by Qian et al. can be used to prepare carbon spheres. During the process, toluene was first vaporized at $200^{\circ} \mathrm{C}$ in the first furnace, then the vapors decomposed over the template at $1100^{\circ} \mathrm{C}[323]$. Similarly, acetylene/toluene was used as the carbon precursors for the preparation of carbon spheres at $1000^{\circ} \mathrm{C}[324]$. Other carbon substrate such as styrene, benzene, hexane, cyclohexane and ethane are also used in the large scale production of carbon spheres by a double furnace[325]. The feedstock was vaporized in the low temperature furnace at $250^{\circ} \mathrm{C}$ and then introduced to the high temperature reactor at range of $900-1200^{\circ} \mathrm{C}$. The size of the synthesized carbon spheres is affected by the feed rate, temperature and reaction time. Not only carbon source contains only carbon and hydrogen can be used for the synthesis of carbon spheres, corn starch, tetrahydrofuran etc. which contain other elements such as oxygen can also be used to produce good quality of carbon spheres[326-328]. The above described procedures are non-catalytic CVD synthesis methods. In fact, metal catalysts are widely used in the production of shaped carbon materials such as carbon nanotube and graphene. These catalysts are also applicable for the synthesis of carbon spheres, acting as a template providing the nucleation sites for the formation of hollow sphere. By using toluene as the carbon source and $\mathrm{ZnSe}$ nanoparticles as the template, hollow carbon spheres were produced with diameters of $40-120 \mathrm{~nm}$ at $1200^{\circ} \mathrm{C}$ after $60 \mathrm{~min}[329]$. Similar procedures were applied for the synthesis of nitrogen doped carbon spheres using fumed silica as hard template and melamine-formaldehyde resin as carbon precursor[330]. Moreover, using silica spheres as template, smooth single sell, deformed single shell and double shell carbon spheres can be 
produced[331]. Large scale synthesis of carbon spheres was reported by Miao et al. using Kaolin supported transition metal salts as catalysts[332, 333]. Monodisperse $150 \mathrm{~nm}$ carbon spheres with a foam-like macroscopic structure were synthesized by CVD methods in which acetylene was used as the carbon sphere and nickel metal clusters acted as catalyst. Hollow carbon nanospheres were synthesized from $\mathrm{CH}_{4}$ using a sol-gel procedure in the presence of $\mathrm{Ni} / \mathrm{Al}_{2} \mathrm{O}_{3}$ in which the $\mathrm{Ni}$ is supposed to act as catalyst/template for the growth of the carbon spheres[334]. For the catalytic chemical vapor deposition method, the separation of the encapsulated catalyst and the carbon spheres is a problem need to consider[335]. Thus mixed valence oxide catalysts were used for the synthesis of carbon sphere in the hope that this would make the separation process easier. Wang and Kang prepared various carbon microspheres using $\mathrm{CH}_{4}$ as carbon source and transition or rare earth metal oxides with mixed valences as catalysts at $1100^{\circ} \mathrm{C}[336]$.

The carbon sphere synthesis method described above are all carried out at very high temperature which is energy intensive and procedures insecure. In order to overcome these disadvantages, autoclave methodologies were developed to synthesize carbon spheres under mild temperature condition. Pressure build up in the enclosed environment during the reaction process and the final reaction products will be affected by this pressure. A range of common organic reagents were used for the synthesis of carbon spheres in autoclaves[337, 338]. A perfect spherical morphology of non-graphitic carbon was produced by using mesitylene as carbon source[337]. The polymer polyethylene terephthalate has also been used as carbon source to prepare carbon spheres in a stainless steel reactor at $700^{\circ} \mathrm{C}[339]$. Further studies on the synthesis of carbon spheres in water using carbohydrates as carbon source greatly reduced the reaction temperature to around $160-200^{\circ} \mathrm{C}[340]$. Hollow carbon spheres can be generated by the combination of hydrothermal carbonization of glucose, polystyrene and P123 and 
microwave treatment of the carbonization products[341]. Silica particles can be used as template during the hydrothermal carbonization of sucrose followed by calcination and HF etching to produce hollow carbon spheres. High quality and uniform size carbon spheres were reported to be synthesized at $65^{\circ} \mathrm{C}$ in the presence of $\mathrm{CaC}_{2}$ and oxalic acid[342]. In fact, catalysts and reducing agents are also widely used for the preparation of carbon spheres in an autoclave. $30-580 \mathrm{~nm}$ both filled and hollow carbon spheres were synthesized by the catalytic pyrolysis of $\mathrm{C}_{2} \mathrm{Cl}_{4}$ in the presence of ferrocene[343]. Similar procedures were carried out by Jia et al. to prepare hollow carbon spheres using ferrocene and ammonium chloride as starting material in argon atmosphere at $700^{\circ} \mathrm{C}[344]$. Neckless-like hollow carbon spheres with size of 50-100nm were synthesized from hexachlorocyclopentadiene and ferrocene at $500^{\circ} \mathrm{C}$ in a stainless steel autoclave under an argon atmosphere[345]. Adding $\mathrm{NaCl}$ into the reaction during the carbonization of hexachlorobenezene can facilitate the formation of hollow carbon spheres because $\mathrm{NaCl}$ can act as a template for the synthesisi of core-shell structure[346]. 400-600nm hollow carbon spheres were synthesized from $\mathrm{CCl}_{4}$ in the presence of $\mathrm{NaNH}_{2}$ with $\mathrm{Co} / \mathrm{Ni}$ alloy as the catalyst[347]. Mg powder and $\mathrm{Na}_{2} \mathrm{CO}_{3}$ can also be used as catalysts for the carbonization of $\mathrm{CCl}_{4}$ to make hollow carbon spheres[348]. Acetone was used to synthesize uniform carbon microspheres in a titanium alloy autoclave by a substrate assisted route in the presence of $\mathrm{Ni}$ foil[349, 350]. Ni catalyst was also applied in the synthesis of coral-like accreted hollow carbon microspheres from cyclohexane[351]. Recently, various mixtures of ferrocene and anthracene were used as starting materials for the synthesis of carbon spheres in evacuated quartz tubes placed in a furnace[352].

Carbon spheres can be used as catalyst supports as most carbon materials. Carbon sphere-supported metal nanoparticles has been widely used in a number of different catalytic reactions. Carbon spheres embedded with iron oxide 
particles showed good activity in the Fischer-Tropsch synthesis[353]. Magnetic $\mathrm{Fe}_{3} \mathrm{O}_{4} /$ carbon sphere/cobalt composites were synthesized an applied for the catalytic oxidation of phenol solutions with sulfate radicals[354]. Carbon spheres supported visible-light-driven $\mathrm{CuO}-\mathrm{BiVO}_{4}$ heterojunction was prepared by hydrothermal process and impregnation technique[355]. The catalyst is an excellent photocatalyst for the degradation of organic pollutants. Liu et al. claimed that carbon spheres supported Pd catalyst can be used for the removal of trace amount of ethylene in agribusiness environments[356].

Carbon spheres are also widely used in electrochemical cells and batteries. Small Pt particles supported on carbon spheres were used for the methanol oxidation in direct methanol fuel cells[357]. High surface area hollow carbon spheres loaded with $\mathrm{Pd}$ particles were used for ethanol oxidation in an electrochemical cell[341]. PtRu supported on hollow carbon spheres were made into an electrode in a proton exchange membrane fuel cell[358]. Recently, carbon spheres supported Ag particles was mixed with Nafion and supported on a glassy carbon electrode for the oxidation of hydrazine[359]. For these applications, the well separated and stable metal particles which provide easy access for the reactant to contact with the active sites is the foundation of the successful electrochemical activity. 


\section{Chapter 3 Techniques of Characterization, Synthesis, and Catalytic Reactions}

Many characterization technologies have been developing for the study of the chemical and physical properties of carbon materials. X-ray diffraction is used to study the composition and the molecular structure of a crystal. Raman spectroscopy is usually used to examine the extent of graphitization of the carbon materials. Fourier transform infrared spectroscopy can provide information on the surface groups of the carbon materials. Element analysis can help to study the elemental composition of the catalysts. X-ray absorption near edge structure is an important technology for the study of the phase, geometrical structures and electronical properties of the catalysts especially for the metal complexes. The structure of a certain compounds can be determined though the peaks of nuclear magnetic resonance spectra. Scanning electron microscope is widely used for the study of the morphologies of catalysts. Thermo gravimetric analyzer is used to study the weight change of the catalyst as temperature changes, it can also be used to determine the loading amount of metal on carbon supports. BrunauerEmmett-Teller method is commonly used to study the surface area, pore size and pore volume of the catalysts. Through these characterization information, we can now better on the nature and arrangement of the building blocks of the catalysts, the morphologies and porosity, which is helpful for the further investigation on the catalytic mechanism and can provide necessary theory support for the catalysts design.

\subsection{Characterization techniques}

\subsubsection{Characterization of catalyst morphologies}

Field emission scanning electron microscopy (FESEM) is widely used for morphological studies which can also give the diameters, shape and chemical compositions of carbon spheres by providing topographical and elemental information at magnifications of 10x to 300,000x, with virtually unlimited depth 
of field. Here, the morphology and structure of the catalysts were investigated using a field emission scanning electron microscope (FESEM, JOEL JSM 6701F). Before measurements, the samples were loaded on a sample holder with adhesive carbon double-sided tape and subsequently deposited with atomic Pt via sputtering.

\subsubsection{Characterization of catalyst structures}

X-ray diffraction (XRD) is a powerful tool for the determination of the crystallinity, inter-layer spacing, structural strain and impurities or supportedmetal particles in carbon spheres[360]. It is a very effective and destructive technology which need little amount of sample for the measurement. For the amorphous carbon, there are usually two broad peaks at around $25^{\circ}$ and $45^{\circ}$ shown on the patterns, in which the width of the peaks gives information on carbon sphere crystallinity[361, 362]. XRD can also be used to study the supported metal nanoparticles. The average size of crystalline particles can be estimated based on the width at half maximum of specific peaks according to the Scherrer's equation[363]. Here, powder X-ray diffraction patterns were recorded on a Bruker Advance $8 \mathrm{X}$-ray diffractometer using a $\mathrm{Ni}$ filtered $\mathrm{Cu} \mathrm{K} \alpha$ radiation $(\lambda=0.154 \mathrm{~nm})$, operated at $40 \mathrm{kV}$ and $40 \mathrm{~mA}$. XRD data were collected between 10 and $90^{\circ}(2 \theta)$ with a resolution of $0.02^{\circ}$.

Raman spectroscopy is an old but useful technology for the characterization of various carbon materials including carbon black, carbon nanotube, graphite, glassy carbon, coal and sputtered carbon. The Raman spectroscopy can reflect the size, strength and arrangement of chemical bonds. The most famous peaks for carbon materials are the so called D band which is around $1350 \mathrm{~cm}-1$ and attributed to residual disorganized graphite, and G band which is around 1580$1600 \mathrm{~cm}-1$ and assigned to graphite carbon[364-366]. The intensity ratio of D band and $\mathrm{G}$ band is a key factor to evaluate the proportion of graphitic and disordered carbon[367]. Here, Raman tests were carried out on a Renishaw 1000 
Raman spectrometer equipped with a 514/633 nm excitation from HeNe laser, of which the detection depth is about $150 \mathrm{~nm}$.

Physical adsorption of nitrogen is a typical technique to measure the surface area, pore size, pore volume and pore sized distribution of carbon materials from the adsorption and desorption isotherms[368, 369]. Here, the BET surface areas were measured on Autosorb-6B (Quantachrome instruments) using the liquid nitrogen adsorption method. Samples were degassed at $150^{\circ} \mathrm{C}$ over night before the measurement.

\subsubsection{Characterization of catalyst active sites}

FTIR spectroscopy has been used to study the chemical composition of carbon materials, especially for the determination of surface functional groups on the carbon supports. Usually, bands at $1630 \mathrm{~cm}-1$ and $2850-2920 \mathrm{~cm}-1$ are the two major sets of bands for carbon spheres, the former is attributed to $\mathrm{C}=\mathrm{C}$ groups and the latter is assigned to $\mathrm{C}-\mathrm{H}$ groups present on the surface of carbon spheres[370]. Other functional groups such as acidic groups, hydroxylic groups, carboxylic groups can also be detected by FTIR. Thus, FTIR can be used to study the changes of the groups on the surface of carbon spheres after the reactions. Also it can provide information on whether a certain function group is successfully grafted on the carbon sphere. In this thesis, one of the work is the dehydration of fructose to HMF by carbon-based solid acid. FTIR can provide evidence for the successful introduction of sulfuric acid groups[371]. Here, FTIR spectra were obtained on Digilab FTS 3100 FTIR with a $4 \mathrm{~cm}-1$ resolution in the range of 400-4000 cm-1 using a standard $\mathrm{KBr}$ disk technique.

The V K-edge X-ray absorption near edge structure (XANES) is a very sensitive and powerful technique to study the metal oxide encapsulated in the carbon spheres[372]. When only a small amount of metal oxide was loaded on the carbon support with homogenous dispersion, it is difficult to see the signal of the metal oxide on the XRD patterns, and X-ray photoelectron spectroscopy is 
not available due to the limitation of its depths of detection. This will be the time for XANES to show its advantages. It can determine the chemical state of elements which are present in bulk in minute quantities. Here, XANES spectra was recorded at XAFCA beamline of Singapore Synchrotron Light Source (SSLS).

\subsubsection{Characterization of chemical properties of catalysts}

As the same suggests Elemental Analysis is a technique for the determination of the mass fraction of carbon, hydrogen, nitrogen and sulfur of the tested samples, which can help to ascertain the structure and purity of a synthesized compound. In this thesis, carbon materials are prepared by different methods and after treated with different conditions. Element analysis was used to study the influence of various reaction conditions on the chemical composition of the synthesized carbon spheres as well as the stability of the functional groups and the carbon supports. Here, the element composition of the catalyst was estimated by Vario EL III CHNS Elemental Analyzer.

Thermo gravimetric analysis is a technique that has been widely used for the characterization of carbon spheres, in which changes in physical and chemical properties of materials are measured as a function of increasing temperature. These data provide basis for the further thermal treatment of the catalyst in order to modify the physicochemical properties of the carbon materials. Beside the study of the thermostability of the catalysts, TGA can also be used to determine the organic or inorganic content in the carbon sphere supported catalysts[373]. In this thesis, carbon sphere encapsulated metal oxide catalysts were synthesized and their TGA tests were run in air under high temperatures to remove the carbon support. Thus the remaining items will be the metal oxide. Here, the TGA test was conducted on an SDT Q600 instrument and alundum pans under air atmosphere. 
Nuclear Magnetic Resonance (NMR) is a widely used and selective technique for the determination of the topology, dynamics and three-dimensional structure of molecules in solid or solution state. Carbon-13 NMR and proton NMR are the most commonly used NMR measurements. NMR can also be used for purity determination[374]. In this thesis, 1H-NMR spectra was measured to analysis the separated product on a Bruker Avance II $300 \mathrm{MHz}$ spectrometer withCDCl3 as the solvent.

Temperature-programmed desorption (TPD) is a widely used method for the observation of desorbed molecules from the surface of the catalysts when the surface temperature is increasing. Small molecules or atoms always absorb onto the surface of the catalysts by forming a bond with the surface to minimized their energy. When the surface temperature increases, the energy transferred to the adsorbed molecules or atoms will cause them to desorb. Then the desorbed species can be identified and quantified by a mass spectrometer to give information on the surface attached functional groups. In addition, $\mathrm{NH}_{3}$ can absorb on the surface of the samples as probe molecules under room temperature and desorb during the temperature programmed increasing. Generally, the adsorption force between $\mathrm{NH}_{3}$ and acidity of the surface of the catalysts has a positive linear relation. High temperature is essential when the force of attraction is so strong that the adsorption cannot be reversed easily. Thus $\mathrm{NH}_{3}-\mathrm{TPD}$ can provide information on the acidity distribution of the catalysts[375]. Here, TPD was performed on a Micromeritics AutoChem II 2920 instrument. Typically, the pretreatment of the sample was conducted in a quartz reactor by high-purity Ar at $200{ }^{\circ} \mathrm{C}$ for $2 \mathrm{~h}$. Then after the temperature cooled down to $100^{\circ} \mathrm{C}$, it was set to rise to $1020^{\circ} \mathrm{C}$ at a rate of $5{ }^{\circ} \mathrm{C} / \mathrm{min}$. A mass spectrometer (ThermoStar GSD 301 T2) was used to detect the desorbed $\mathrm{CO}$ and $\mathrm{CO}_{2}$. The ammonia temperatureprogrammed desorption (NH3-TPD) was carried out on a Micrometrics Auto 
Chem 2920 chemisorption analyzer equipped with a quadrupole mass spectrometer to study the acidity of the catalyst.

\subsection{Synthesis methodologies}

\subsubsection{Materials}

Concentrated nitric acid (69\%, Sigma-Aldrich), alpha-D (+)-glucose (Sigma, 99.5\%, anhydrous), chloric acid $\mathrm{HCl}$ (36-38 wt.\%), potassium hydroxide $\mathrm{KOH}$ (90\%, Sigma-Aldrich), sodium hydroxide $\mathrm{NaOH}$ (>98\%, Sigma-Aldrich), toluene anhydrous (>99.8\%, Sigma-Aldrich), ethanol (>99.5\%, Sigma-Aldrich), oxalic acid (OXA, 98\%), ethyl acetate (EA, >99.8\%, Sigma-Aldrich), Fructose ( $\geq 99 \%$, Sigma-Aldrich) , HMF (99\%, Sigma-Aldrich) , DFF(99, SigmaAldrich), HMFCA (99\%, Sigma-Aldrich), FDA (99\%, Sigma-Aldrich), sulfuric acid (98\% purity), DMSO (99\%, Sigma-Aldrich), phosphomolybdic acid (99\%, Sigma-Aldrich), $\mathrm{NH}_{4} \mathrm{VO}_{3}(99 \%$, Sigma-Aldrich). These commercial chemicals were used as received without further purification.

\subsubsection{Preparation of sulfonated carbon sphere solid acid catalysts}

The CS was prepared by hydrothermal carbonization of glucose. In the typical synthesis, $5 \mathrm{~g}$ of glucose was dissolved in $30 \mathrm{ml}$ of deionized water to form a clear solution under stirring. The solution was then transferred into a $40 \mathrm{ml}$ capacity teflon-lined autoclave and maintained at $180^{\circ} \mathrm{C}$ for $10 \mathrm{~h}$. The resulting solid products were filtered and washed with deionized water and ethyl alcohol, followed by vacuum drying for $4 \mathrm{~h}$ at $60^{\circ} \mathrm{C}$ to afford CS.

The as-synthesized CS was dispersed in a sulfuric acid solution under stirring. The suspension was placed in a $40 \mathrm{ml}$ teflon-sealed autoclave and maintained at $180^{\circ} \mathrm{C}$ for $4 \mathrm{~h}$. The black products were filtered, washed and then dried following the same procedures in CS preparation. Sulfuric acid solutions with different concentrations were employed in the sulfonation processes. The sulfonated CS solid acid catalysts were labeled as CS-1, CS-2, CS-3 and CS-4 according to the sulfuric acid and water volumetric ratios of 1:5, 1:2, 1:1 and 2:1, respectively. 
For comparison purposes, sulfonated CS was also prepared in one-step following the procedures as reported by Liu et al[376]. Equal amounts of glucose dissolved in sulfuric acid solutions of different sulfuric acid and water ratios (1:5, 1:2, 1:1, 2:1); the solutions were placed in teflon-sealed autoclaves and heated at $180{ }^{\circ} \mathrm{C}$ for $4 \mathrm{~h}$. The products were labeled as C-1, C-2, C-3 and C-4.

\subsubsection{Preparation of V-embedded mesoporous carbon microspheres}

The V-embedded mesoporous carbon microspheres were prepared by one step hydrothermal method of glucose and $\mathrm{NH}_{4} \mathrm{VO}_{3}$ solution followed by calcination. Typically, $\mathrm{NH}_{4} \mathrm{VO}_{3}$ and oxalic acid (molar ratio 1:2) were dissolved in $30 \mathrm{~mL}$ DI water and kept stirring to form dark green solution. Then $4 \mathrm{~g}$ glucose was added into the solution and stirred for $3 \mathrm{~h}$. Afterward, the solution was transferred to a Teflon-lined autoclave ( $40 \mathrm{~mL}$ in volume) and hydrothermally treated in an electric oven at $180^{\circ} \mathrm{C}$ for $4 \mathrm{~h}$, and naturally cooled to room temperature, the dark product precipitate was collected by filtering and washing thoroughly with water and ethanol and then dried at $60^{\circ} \mathrm{C}$ overnight. This assynthesized sample was denoted as V-CS. The V-CS sample was then transferred into a crucible with a lid and calcined at different temperatures $(600,700,800$, $900{ }^{\circ} \mathrm{C}$ ) for $1 \mathrm{~h}$ in a muffle furnace connected to the outside atmosphere or in a tube furnace under $\operatorname{Ar}$ at $900^{\circ} \mathrm{C}$. These obtained black powders were marked as V-CS-600, V-CS-700, V-CS-800, V-CS-900 and V-CS-900-Ar.

\subsubsection{Preparation of molybdenum trioxide supported on carbon spheres}

The Mo-carbon microspheres were prepared by a classical hydrothermal carbonization procedure, in which glucose and phosphomolybdic acid were used and the carbon and Mo sources respectively. Typically, glucose and phosphomolybdic acid were dissolved in $30 \mathrm{~mL}$ DI water with molar ratio of carbohydrate to metal was $8: 1$. and kept stirring to 6 h until the color of the solution turn from yellow to green. Afterward, the solution was transferred to a Teflon-lined autoclave (40 mL in volume) and put into a constant temperature 
oven of $180^{\circ} \mathrm{C}$ for $12 \mathrm{~h}$. Then brown or dark product was collected by filtering and washing thoroughly with water and ethanol after the autoclave naturally cooled to room temperature and then dried in vacuum overnight. This assynthesized sample was denoted as Mo-CS. The Mo-CS sample was then transferred into a muffle furnace which was connected to the outside atmosphere and calcined at $275^{\circ} \mathrm{C}$ for $3 \mathrm{~h}$, the resulted sample was marked as Mo-CS-275. The Mo-CS sample was also annealing under nitrogen in a tube furnace at $750^{\circ} \mathrm{C}$ for $3 \mathrm{~h}$, the resultant sample was donated as Mo-CS-N2.

\subsection{Catalytic reactions}

\subsubsection{Dehydration of fructose to $\mathrm{HMF}$}

The catalytic reaction was performed in a $25 \mathrm{ml}$ capacity flask equipped with a condenser. In a typical catalytic run, a DMSO solution of fructose $(0.5 \mathrm{~g}$ of fructose in $10 \mathrm{ml}$ of DMSO) was added into the flask along with $100 \mathrm{mg}$ of CS solid acid catalysts. The reaction mixture was heated to the reaction temperature in oil bath with a thermostat and an electronically controlled magnetic stirrer. The reaction samples were removed periodically and analyzed by liquid chromatography (HPLC, Agilent 1100 series, Bio-Rad Aminex HPX-87H, $300 \mathrm{~mm} \times 7.8 \mathrm{~mm}$ pre-packed column). Reactant conversion (mol \%), HMF yield (mol \%) and product selectivity (\%) were defined as follows:

$$
\begin{aligned}
& \text { Conversion }(\mathrm{mol} \%)=\frac{\text { moles of fructose reacted }}{\text { moles of initial fructose }} \times 100 \% \\
& \text { Yield }(\text { mol } \%)=\frac{\text { moles of HMF produced }}{\text { moles of initial fructose }} \times 100 \% \\
& \text { Selectivity }(\%)=\frac{\text { moles of HMF produced }}{\text { moles of fructose reacted }} \times 100 \%
\end{aligned}
$$

\subsubsection{Selective oxidation of HMF to DFF}

Typically, HMF (63mg, 0.5mmol) was firstly dissolved into $5 \mathrm{~mL}$ solvent with a magnetic stirring at room temperature. Then the catalyst was added and oxygen was bubbled through the reaction mixture at a flow rate of $20 \mathrm{~mL} / \mathrm{min}$. The reaction was conducted at different temperatures (i.e., $100,120,140,160^{\circ} \mathrm{C}$ ). 
The reactor was equipped with a condenser to prevent the evaporation of the solvent. After reaction, the catalyst was separated from the reaction mixture by filtration and products were analyzed by HPLC.

\subsubsection{One-pot and One-step synthesis of DFF from fructose}

\section{Dehydration of fructose to HMF}

In a typical reaction, the procedure for fructose dehydration into HMF was as follows:

Fructose (200mg), Mo-carbon catalyst (30mg), and DMSO (5mL) were added into a $25 \mathrm{~mL}$ three-necked, round-bottomed flask equipped with a reflux condenser. The reaction was heated in oil bath with magnetic stirring. After the reaction, the mixture was filtered and diluted with water before analyzed by HPLC.

\section{Aerobic oxidation of HMF to DFF}

In a typical run, the procedure for aerobic oxidation of HMF was as follows: HMF (63mg, 0.5mmol), Mo-carbon catalyst (30mg), and DMSO (5mL) were added to a 25 three-necked round-bottomed flask equipped with a reflux condenser and magnetic stirring. The reaction was heated in oil bath to target temperature and bubbled with $\mathrm{O}_{2}$ at a flow rate of $20 \mathrm{~mL} / \mathrm{min}$. After the reaction, the mixture was filtered and diluted with water and then analyzed by HPLC.

\section{Direct conversion of fructose to DFF}

Typically, fructose (200mg) was firstly dissolved into $5 \mathrm{~mL}$ DMSO with a magnetic stirring at room temperature. Then the catalyst was added and oxygen was bubbled through the reaction mixture at a flow rate of $20 \mathrm{~mL} / \mathrm{min}$. The reaction was conducted at different temperatures (i.e., $100,120,140,160^{\circ} \mathrm{C}$ ). The reactor was equipped with a condenser to prevent the evaporation of the solvent. After reaction, the catalyst was separated from the reaction mixture by filtration and products were diluted with water before analyzed by HPLC.

\subsubsection{Analytical Methods}


Analyses of fructose, HMF and DFF were conducted on an Aglient 1260 HPLC system with a Bio-rad aminex $87 \mathrm{H}$ column $(300 \mathrm{~mm} \times 7.8 \mathrm{~mm}$ pre-packed column) for the separation of the samples components[133, 377]. The reaction products were diluted in water and filtered through a syringe filter (VWR, 0.22 um PTFE) prior to analysis. The mobile phase was constituted of $5 \mathrm{mM} \mathrm{H}_{2} \mathrm{SO}_{4}$ solution at $0.6 \mathrm{~mL} / \mathrm{min}$. The column oven temperature was kept at $60^{\circ} \mathrm{C}$. The amount of each compound was quantified by external standard calibration curve method, which were constructed based on the pure compound. 


\section{Chapter 4 Efficient Dehydration of Fructose to 5-hydroxymethylfurfural over Sulfonated Carbon Sphere Solid Acid Catalysts}

In this work, a modified preparation of carbon-based solid acid under mild conditions was developed. The CS was prepared by hydrothermal carbonization of glucose at $180{ }^{\circ} \mathrm{C}$ which was remarkably lower than the temperature in other CS synthetic routes. The resulted CS was sulfonated by sulfuric acid aqueous solutions instead of concentrated $\mathrm{H}_{2} \mathrm{SO}_{4}$ or fuming sulfuric acid. Catalytic results showed that the catalysts afforded high activity for the dehydration of fructose to HMF. The effects of acid site density, reaction time, solvents, catalyst amount, temperature and mole ratio of catalyst to substrate were investigated. Under an optimized condition, fructose was converted into $\mathrm{HMF}$ with $90 \%$ yield at $160{ }^{\circ} \mathrm{C}$ after reaction duration of $1.5 \mathrm{~h}$.

\subsection{Characterization of CS catalysts}

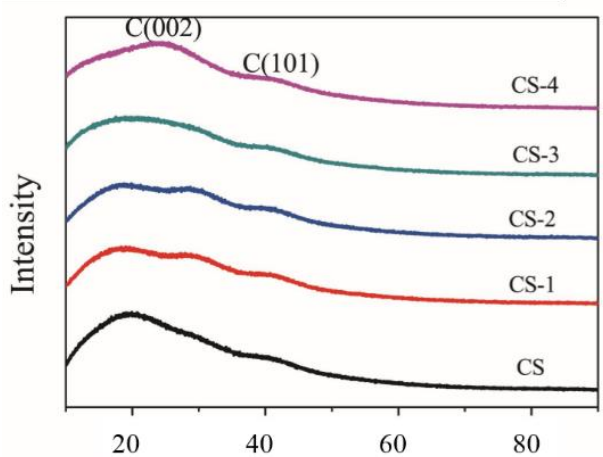

(a) $2 \theta$ (Degree)

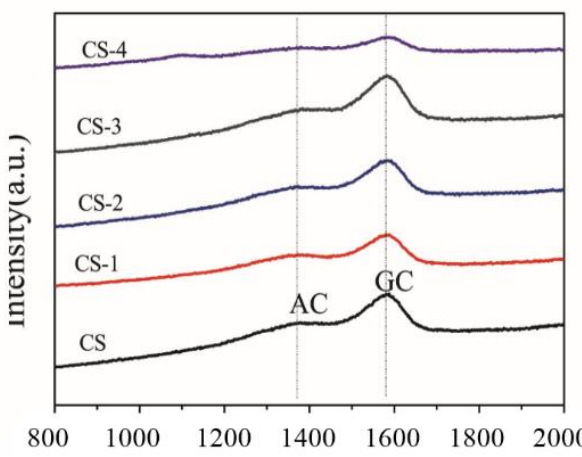

(b)Raman shift $\left(\mathrm{cm}^{-1}\right)$

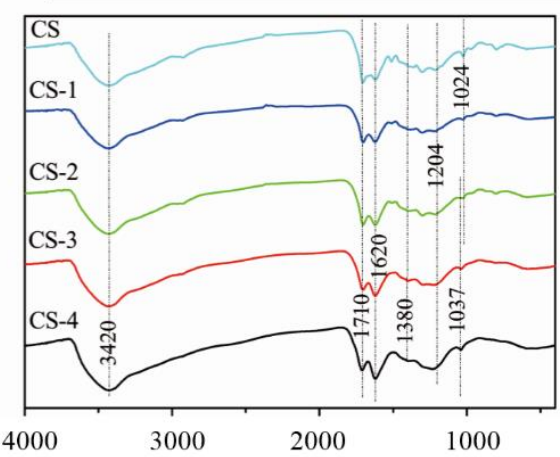

(c)Wavenumbers $\left(\mathrm{cm}^{-1}\right)$

Fig. 4.1 (a) XRD patterns of CS, CS-1, CS-2, CS-3 and CS-4, (b) Raman spectra of CS, CS-1, CS-2, CS-3 and CS-4, (c) FTIR spectra of CS, CS-1, CS-2, CS-3 and CS-4

The XRD patterns of synthesized CS solid acids are shown in Fig. 4.1(a). The weak diffraction peaks at $2 \theta$ angles of $10-30^{\circ}$ and $35-50^{\circ}$ are attributed to the (002) and (101) planes of amorphous carbon, respectively, indicating the carbonization of the glucose precursors [204, 216]. All the samples display two broad signals D-band (1390 cm $\mathrm{cm}^{-1}, \mathrm{~A}_{1 \mathrm{~g}} \mathrm{D}$ breathing mode) and G-band $\left(1590 \mathrm{~cm}^{-1}, \mathrm{E}_{2 \mathrm{~g}} \mathrm{G}\right.$ mode) 
in the Raman spectra as shown in Fig. 4.1(b). The peak intensity ratios of D- to G-band of these CS materials are nearly the same, implying the similar average graphene sizes of these CSs [378]. The FTIR spectra of these CS samples are shown in Fig. 4.1(3). The absorption band at $1037 \mathrm{~cm}^{-1}$ is assigned to $-\mathrm{SO}_{3} \mathrm{H}$ groups, showing that the $-\mathrm{SO}_{3} \mathrm{H}$ groups are successfully linked onto the $\mathrm{CS}$ surfaces. The bands attributed to -OH group at $3420 \mathrm{~cm}^{-1}, \mathrm{C}=\mathrm{O}$ group at $1720 \mathrm{~cm}^{-}$ ${ }^{1}$ and $\mathrm{C}-\mathrm{O}$ group at $1203 \mathrm{~cm}^{-1}$ suggest the presence of $-\mathrm{COOH}$ groups. The peaks at 1620 and $1380 \mathrm{~cm}^{-1}$ are attributed to $\mathrm{C}=\mathrm{C}$ groups.
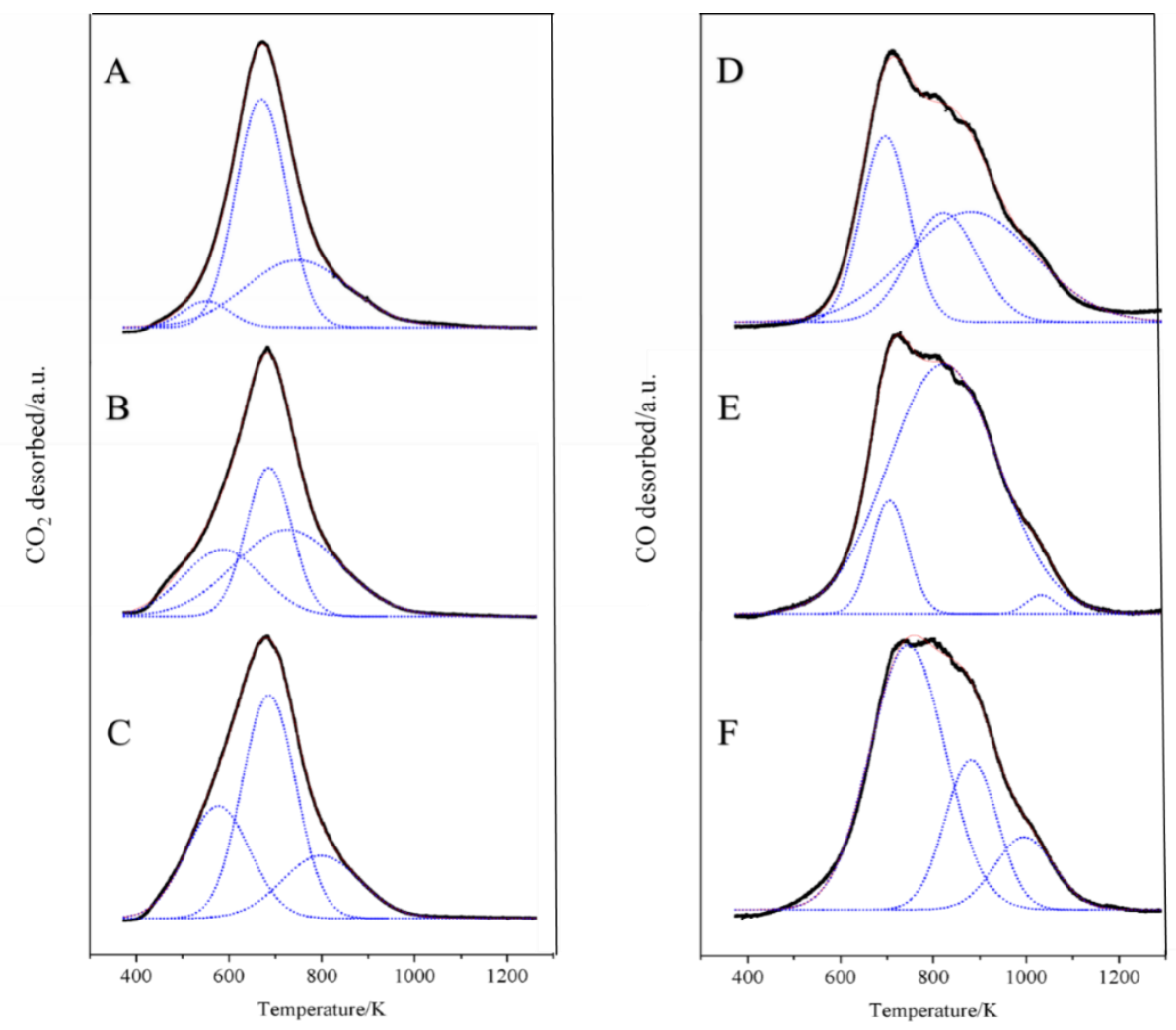

Fig. 4.2 TPD profiles for carbon spheres. A-C CO2 desorption profiles, D-F $\mathrm{CO}$ desorption profiles. $\mathrm{A}$ and $\mathrm{D}$ are carbon spheres before sulfonation, $\mathrm{B}$ and $\mathrm{E}$ are carbon spheres after sulfonation, $\mathrm{C}$ and $\mathrm{F}$ are sulfonated carbon spheres recycled after dehydration reaction.

The oxygen-containing functional groups on the surfaces are further studied by TPD and the relative concentrations of various functional groups including carboxyl, anhydride, ester, phenol and carbonyl/quinone are shown in Fig.4.2, 
and summarized in Table 4.1. The sequence of temperatures for decomposition of the oxygen-containing groups to produce $\mathrm{CO}_{2}$ and $\mathrm{CO}$ upon heating is carboxyl < anhydride < ester and anhydride < phenol < carbonyl/quinone. The relative concentrations of different functional groups are estimated by means of overlapping peak deconvolution.

Table 4.1 Relative concentration of functional groups over the CS catalysts ${ }^{\mathrm{a}}$

\begin{tabular}{llll}
\hline Functional groups & CS & CS acid & CS acid $^{\mathrm{b}}$ \\
\hline Carboxyl & 17 & 73 & \\
Anhydride & 97 & 56 & 100 \\
Ester & 77 & 100 & 100 \\
& CO desorption peak & 53 \\
Anhydride & 45 & 18 & \\
Phenol & 32 & 100 & 100 \\
Carbonyl/quinone & 100 & 3 & 34 \\
\hline
\end{tabular}

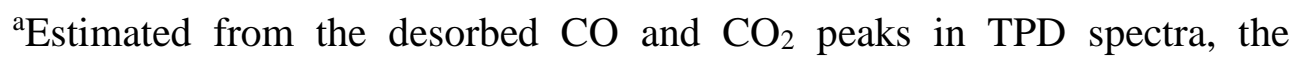
concentration of the strongest peak for each functional groups among the three samples has been normalized to $100 .{ }^{b}$ The catalyst sample was collected after reaction.

As seen in Table 4.1, for the CS after sulfonation, the relative concentrations of carboxyl, ester and phenol groups increase while those of anhydride and carbonyl/quinone decrease. After reaction, the concentrations of carbonyl/quinone and carboxyl groups increase due to the hydrolysis of ester groups and the loss of phenol groups. However, the catalytic reaction results and recycle experiments suggest that the catalytic activities remain the same although the type and quantity of these functional groups change. 

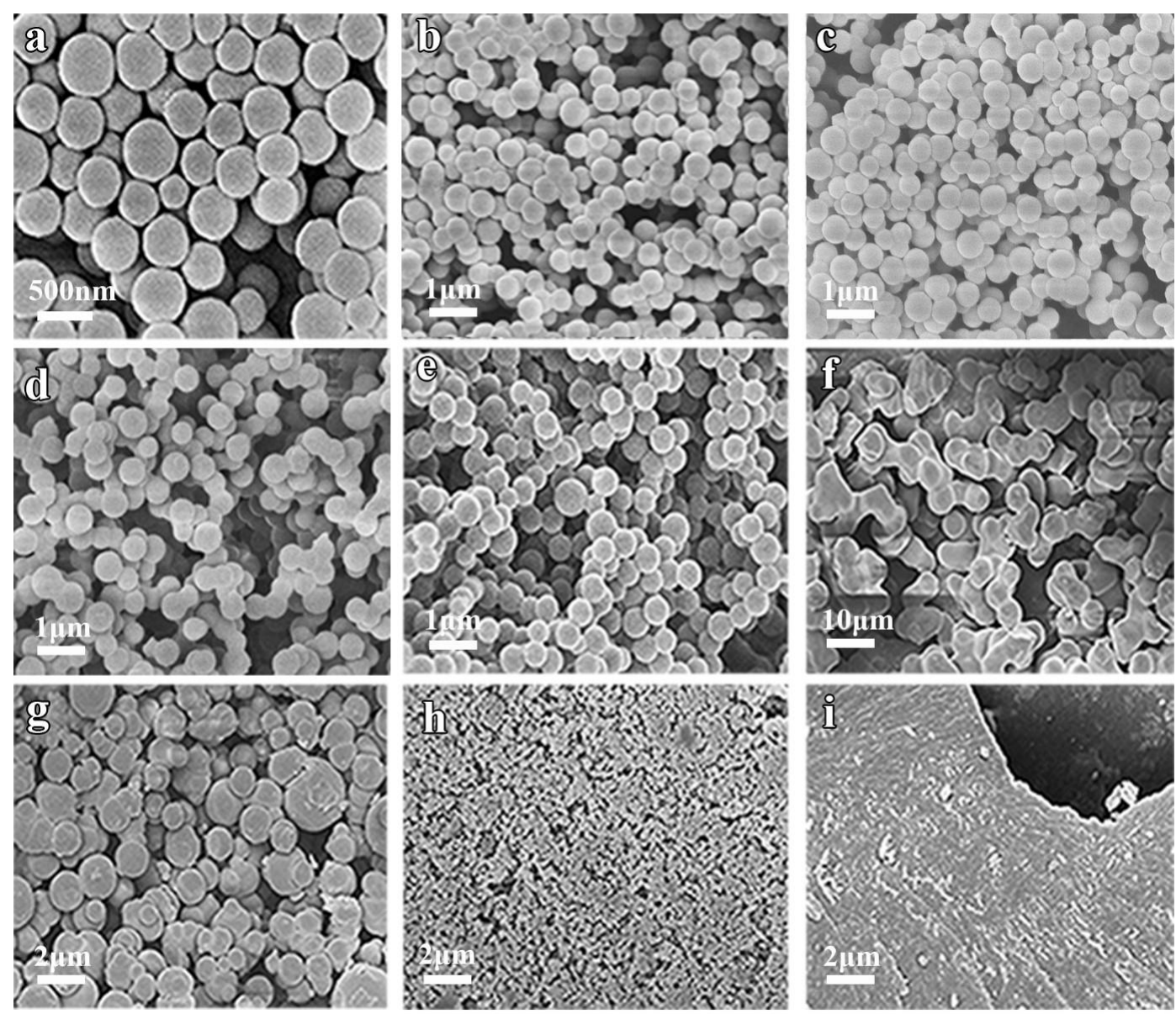

Fig.4.3 FESEM images of: (a) CS, (b) CS-1, (c) CS-2, (d) CS-3, (e) CS-4, (f) C-1, (g) C-2, (h) C-3, (i) C-4.

SEM images shown in Fig. 4.3 reveal that the CSs prepared by the two-step method possess narrow distributed granular diameters of approximately 400-500 $\mathrm{nm}$ regardless the concentration of sulfuric acid solutions. On the other hand, those CSs prepared by the one-step method show irregular shapes with wide size distributions. Furthermore, particle agglomeration occurs significantly on the CSs prepared by the one-step method. The products C-1, C-2, C-3 and C-4 prepared by the one-step hydrothermal method show different morphological features depending on the concentration of the sulfonating solution. The diameter of $\mathrm{C}-1$ sphere is in the range of $5-8 \mu \mathrm{m}$ while it decreases to $1-4 \mu \mathrm{m}$ as the concentration of the sulfonating solution is doubled (C-2). C-3 and C-4 are 
primarily formed by the aggregation of fine particles and the particle sizes of $\mathrm{C}$ 4 are remarkably smaller than those of C-3.

Table 4.2 The physicochemical properties of the catalysts

\begin{tabular}{cccc}
\hline & $\begin{array}{c}\text { Surface } \\
\text { area }\left(\mathrm{m}^{2} / \mathrm{g}\right)\end{array}$ & $\begin{array}{c}\mathrm{SO}_{3} \mathrm{H} \\
\text { amount(mmol/g) }\end{array}$ & $\begin{array}{c}\text { Acid } \\
\text { density }(\mathrm{mmol} / \mathrm{g})\end{array}$ \\
\hline $\mathrm{CS}-1$ & 26.70 & 0.09 & 8.53 \\
$\mathrm{CS}-2$ & 40.17 & 0.17 & 7.90 \\
$\mathrm{CS}-3$ & 153.75 & 0.32 & 7.89 \\
$\mathrm{CS}-4$ & 357.43 & 0.71 & 7.79 \\
\hline
\end{tabular}

The strength and density of acid sites of carbon-based solid acid is a vital factor closely related to the catalytic activity [379]. The total number of acid sites on the catalyst surfaces were determined by a well-established acid-base back neutralization titration method [380]. The CS catalyst (30 mg) was suspended in a sodium hydroxide aqueous solution $(0.1 \mathrm{M}, 20 \mathrm{ml})$. The mixture was stirred using a magnetic stirrer for $4 \mathrm{~h}$, followed by ultrasonication for $1 \mathrm{~h}$ at room temperature. The concentration of the $\mathrm{OH}^{-}$ions in the supernatant solution was calibrated by oxalic acid standard solution. The content of $-\mathrm{SO}_{3} \mathrm{H}$ groups on the surfaces of the CSs were determined by neutralization titration with sodium hydroxide. A sodium chloride aqueous solution $(0.2 \mathrm{M}, 20 \mathrm{ml})$ was added to the catalyst $(30 \mathrm{mg})$. The mixture was stirred using a magnetic stirrer for $4 \mathrm{~h}$, followed by ultrasonication for $1 \mathrm{~h}$ at room temperature. The supernatant solution was titrated using a sodium hydroxide $(0.01 \mathrm{M})$ solution. The sulfonated CSs have both strong and weak acid sites on the surfaces. As shown in Table 2, the total acid density on the surface is almost the same for CS-1, CS-2, CS-3 and CS4 regardless the concentration of sulfonating solution, while the amount of $-\mathrm{SO}_{3} \mathrm{H}$ increases from CS-1 to CS-4 with increasing the concentration of the sulfuric acid solutions. 
The BET surface areas of CSs also show correlation with the concentrations of the sulfonating solutions as shown in Table 4.2; the areas increase from 26.7 to $357.4 \mathrm{~m}^{2} / \mathrm{g}$ as the sulfuric acid concentrations increase. The BET surface areas of the CSs synthesized by one-step hydrothermal carbonization are remarkably smaller compared to those of prepared by the two-step method [376].

\subsection{Catalytic performance of catalysts}

\subsubsection{Effect of solvent}

Solvents usually play a significant role in liquid phase catalytic reactions and in this study the solvent effects on the dehydration of fructose to HMF are shown in Fig. 4.4. Among the solvents tested, DMSO is the best for fructose dehydration to HMF, showing superior catalytic performances. Amarasekara et al. [381] suggested that DMSO acted as the catalyst in the process of fructose dehydration. To verify the solvent effect of DMSO, a blank experiment in the absence of catalyst is conducted in DMSO. Fructose conversion of $56.4 \%$ and $\mathrm{HMF}$ selectivity of $22.4 \%$ can be achieved in $0.5 \mathrm{~h}$ at $140{ }^{\circ} \mathrm{C}$. Furthermore, by using DMSO and DMF mixture (volume ratio of 1:4) as solvent, $93.1 \%$ of fructose conversion and $52.2 \%$ of HMF yield are obtained in the presence of CS-2 catalyst, while these two values are only $29.3 \%$ and less than $2 \%$, respectively without catalysts. Reactions in toluene and ethylene glycol show extremely high fructose conversion (99.1\% and $82.7 \%$, respectively). Nonetheless, the HMF selectivity is less than $2 \%$ after $2 \mathrm{~h}$ reaction duration, implying the presence of significant side reactions. Caratzoulas and Vlachos [382] studied the solvent effects using hybrid Quantum Mechanics/Molecular Mechanics Molecular Dynamics simulations and found that the activation energy for the hydride transfer in fructose dehydration was closely related with the polar solvent environment. According to the further study by Vlachos et al. [383], DMSO solvents arranged themselves in the immediate vicinity of hydrogen atoms of the hydroxyl groups in fructose, helped prevent fructose from forming reversion and polymerization 
products. On the other hand, DMSO molecules around $\mathrm{C} 1$ atom of HMF hindered the rehydration of HMF and humins formation.

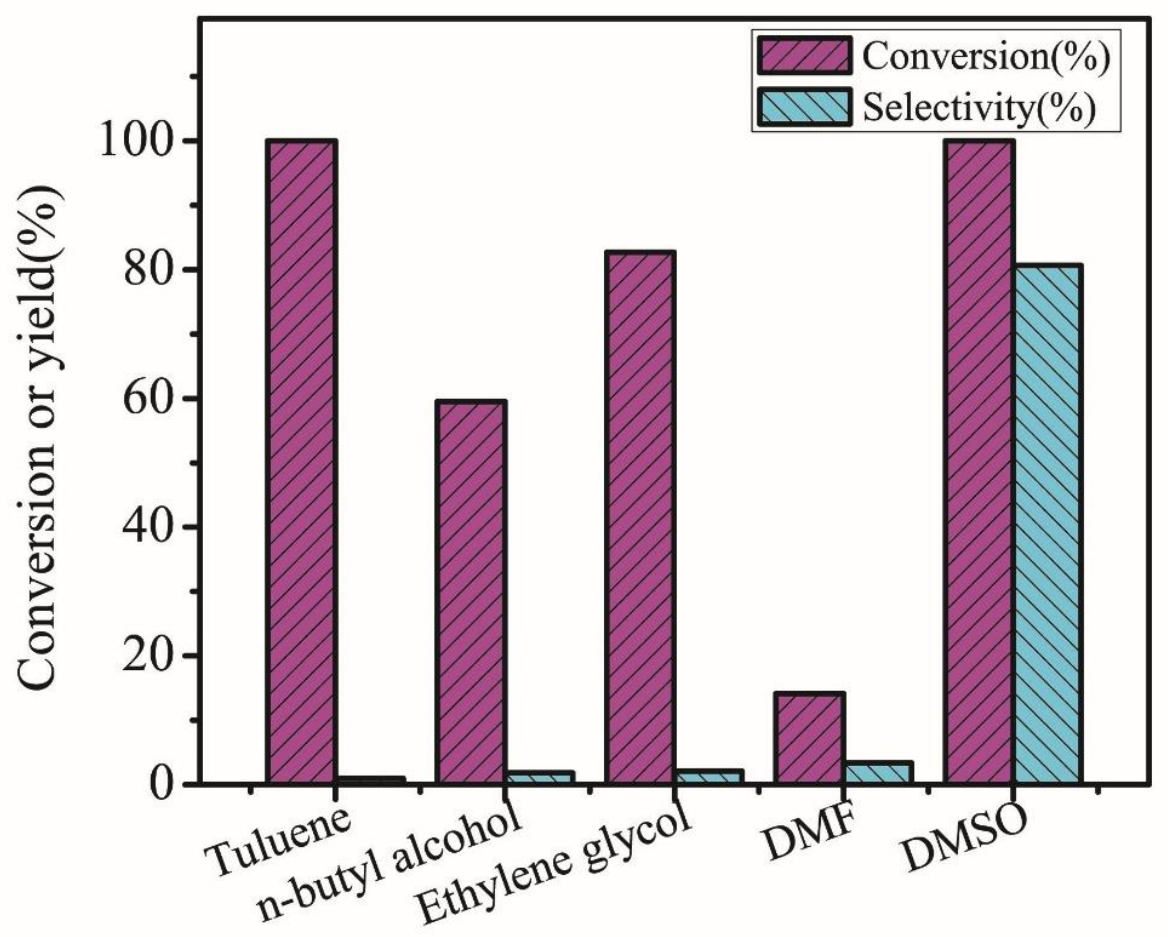

Fig.4.4 Dehydration of fructose in different solvents. Reaction conditions: fructose $(500 \mathrm{mg})$, catalyst $(100 \mathrm{mg})$, solvent $(10 \mathrm{~mL}), 140{ }^{\circ} \mathrm{C}$ for $2 \mathrm{~h}$.

\subsubsection{Effect of the acid site density over the catalyst}

CS catalysts with different amounts of $-\mathrm{SO}_{3} \mathrm{H}$ groups on surfaces are attempted in the dehydration of fructose to HMF, and the results are shown in Fig. 4.5. Amberlyst-15 and $\mathrm{SBA}-\mathrm{SO}_{3} \mathrm{H}$ have been reported as solid acid catalysts to catalyze fructose dehydration to HMF [384], and sulfonic acid groups were suggested as the active sites in this particular reaction. Nonetheless, it can be seen from Fig. 4.5, CS catalyst in the absence of any sulfonic acid groups shows a reasonably good catalytic performance with fructose conversion of $64.3 \%$ and HMF yield of $36.7 \%$ in $0.5 \mathrm{~h}$ reaction duration, implying that other surface acid groups such as $-\mathrm{COOH}$ also show catalytic activity in this reaction [69]. Introducing sulfonic acid groups significantly improves the catalytic activity. The fructose conversion and HMF yield are above $97 \%$ and $75 \%$, respectively over 
CS-2 and CS-3. CS-2 affords the highest HMF selectivity of 77\%. Under the same reaction conditions, a fructose conversion of only $9 \%$ and HMF yield of 3\% are obtained when the reaction is catalyzed by CS-Na which is prepared by ionexchange $\mathrm{H}^{+}$by $\mathrm{Na}^{+}$over $\mathrm{CS}-2$, indicating the important role of protonic acid in the dehydration of fructose to HMF. Generally, the more the sulfonic groups on CS catalyst surfaces, the higher the conversion and yield of catalytic performances, as shown in Fig. 4.5 over catalysts from CS-1 to CS-3. As for CS4, the yield of HMF decreases slightly because HMF proceeds further decomposition due to the remarkably high sulfonic acid site density over CS-4 surfaces, the amounts of sulfonic acid groups of CS-4 is two times higher than that of CS-3 [385]. Furthermore, the conversion of sucrose to HMF is also conducted using the CS solid acid as catalyst and 91.1\% HMF yield can be reached in $6 \mathrm{~h}$ in DMSO at $140^{\circ} \mathrm{C}$, indicating the superior catalytic activity of CS solid acid catalysts.

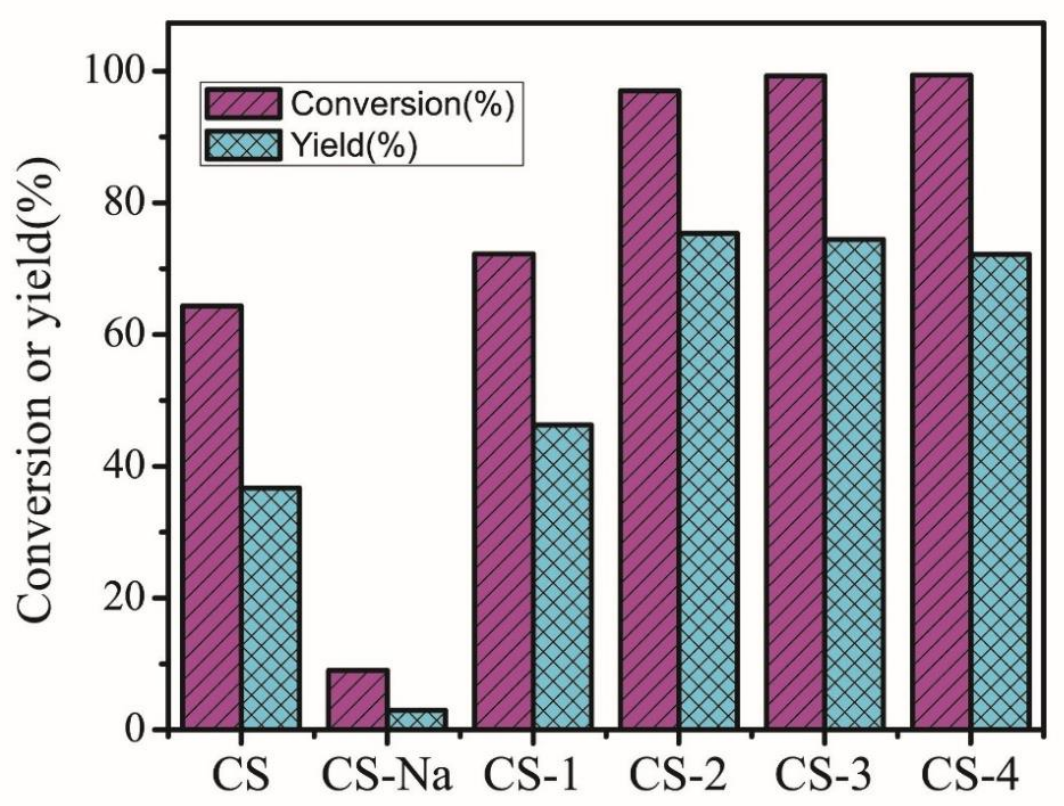

Fig.4.5 Dehydration of fructose by different catalysts. Reaction conditions: fructose $(500 \mathrm{mg})$, catalyst $(100 \mathrm{mg})$, DMSO $(10 \mathrm{~mL}), 140{ }^{\circ} \mathrm{C}$ for $0.5 \mathrm{~h}$.

\subsubsection{Catalytic role of the $S=O$ group}


Table 4.3 The catalytic reaction results for the dehydration of fructose to HMF with different acid catalysts and salts

\begin{tabular}{|c|c|c|c|c|c|c|c|}
\hline Entry & Catalys & Salt & $\mathrm{T}\left({ }^{\circ} \mathrm{C}\right)$ & $\begin{array}{l}\text { Time( } \\
\text { min) }\end{array}$ & $\begin{array}{l}\text { Fructos } \\
\text { e conv. (\%) }\end{array}$ & $\begin{array}{r}\text { HMF } \\
\text { sel. (\%) }\end{array}$ & $\begin{array}{r}\text { HMF } \\
\text { yield }(\%)\end{array}$ \\
\hline 1 & $\mathrm{H}_{2} \mathrm{SO}_{4}$ & - & 125 & 30 & 85.34 & 79.05 & 67.46 \\
\hline 2 & $\mathrm{H}_{2} \mathrm{SO}_{4}$ & $\mathrm{Na}_{2} \mathrm{SO}_{4}$ & 125 & 30 & 76.91 & 68.50 & 52.68 \\
\hline $3^{\mathrm{a}}$ & $\mathrm{H}_{2} \mathrm{SO}_{4}$ & $\mathrm{Na}_{2} \mathrm{SO}_{4}$ & 125 & 30 & 65.05 & 63.98 & 41.62 \\
\hline 4 & CS-2 & - & 125 & 30 & 86.30 & 71.94 & 62.08 \\
\hline 5 & $\mathrm{CS}-2$ & $\mathrm{Na}_{2} \mathrm{SO}_{4}$ & 125 & 30 & 63.63 & 67.36 & 42.86 \\
\hline 6 & CS-2 & $\begin{array}{l}\mathrm{C}_{8} \mathrm{H}_{7} \mathrm{~S} \\
\mathrm{O}_{3} \mathrm{Na}\end{array}$ & 125 & 30 & 93.09 & 70.03 & 65.19 \\
\hline 7 & - & $\mathrm{Na}_{2} \mathrm{SO}_{4}$ & 125 & 30 & 3.04 & - & - \\
\hline 8 & - & $\begin{array}{l}\mathrm{C}_{8} \mathrm{H}_{7} \mathrm{~S} \\
\mathrm{O}_{3} \mathrm{Na}\end{array}$ & 125 & 30 & 34.59 & 35.36 & 12.23 \\
\hline
\end{tabular}

${ }^{a}$ the amount of $\mathrm{Na}_{2} \mathrm{SO}_{4}$ of entry is two times of entry 2.

DMSO acted as both solvent and catalyst in the dehydration of fructose to $\mathrm{HMF}$ and $\mathrm{S}=\mathrm{O}$ group in DMSO was proposed to be the catalytic active site [381]. In this study, different type and quantity of salts in which there are $\mathrm{S}=\mathrm{O}$ structures are added into the reaction system as the control experiments to further verify the role of sulfonic groups on the catalytic reactions (Table 4.3). When adding $\mathrm{H}_{2} \mathrm{SO}_{4}$ as the catalyst, fructose conversion of $85.34 \%$ and HMF yield of $67.46 \%$ are achieved (Table 4.3, Entry 1), while adding $\mathrm{Na}_{2} \mathrm{SO}_{4}$ results in the decrease both in fructose conversion and HMF selectivity (Table 4.3, Entry 2), and the further decrease occurs when more $\mathrm{Na}_{2} \mathrm{SO}_{4}$ is added (Table 4.3, Entry 3). Similar results are observed when using $\mathrm{CS}$ as the catalyst instead of $\mathrm{H}_{2} \mathrm{SO}_{4}$. Fructose conversion decreases from $86.3 \%$ to $63.63 \%$ and the yield of HMF decreases from $62.08 \%$ to $42.86 \%$ due to the addition of $\mathrm{Na}_{2} \mathrm{SO}_{4}$ (Table 4.3, Entry 4, 5). These results suggest that the $\mathrm{S}=\mathrm{O}$ structure in $-\mathrm{SO}_{4}{ }^{2-}$ shows negative effect in 
converting fructose to HMF, resulting in the production of humins and levulinic acid. However, the addition of styrene-4-sulfonic acid sodium salt in the reaction system catalyzed by CS solid acid enhances the catalytic efficiency (Table 4.3, Entry 6). Contrast experiments (Table 4.3, Entry 7, 8) show that styrene-4sulfonic acid sodium has more remarkable catalytic effects than $\mathrm{Na}_{2} \mathrm{SO}_{4}$. Therefore, the $\mathrm{S}=\mathrm{O}$ structure in $-\mathrm{SO}_{3}{ }^{-}$acts as a co-catalyst in the reaction, and the $-\mathrm{SO}_{3}{ }^{-}$group on the surface of the $\mathrm{CS}$ acid promotes the reaction to certain extent.

\subsubsection{Effect of catalyst/substrate ratio}

A series of experiments are conducted at different catalyst/substrate ratios $(5 \%, 10 \%, 20 \%, 30 \%$, and $40 \%)$ at $140{ }^{\circ} \mathrm{C}$ in DMSO using CS-2 as a representative catalyst, and the results are presented in Fig. 4.6. Gradually, the conversion increases from $86.5 \%$ to $100 \%$ as the CS-2/fructose ratio increases from $5 \%$ to $40 \%$, while the HMF yield firstly increases from $60.7 \%$ (catalyst/substrate ratios $5 \%$ ) to $75.4 \%$ (catalyst/substrate ratios $20 \%$ ), followed by slightly decreasing to $70.8 \%$ (catalyst/substrate ratios $40 \%$ ). This decrease in yield is attributed to the increasing amounts of catalyst which causes abundant acid sites in the reaction environment, and the side-reactions such as converting HMF to levulinic acid occurs under such conditions [385]. 


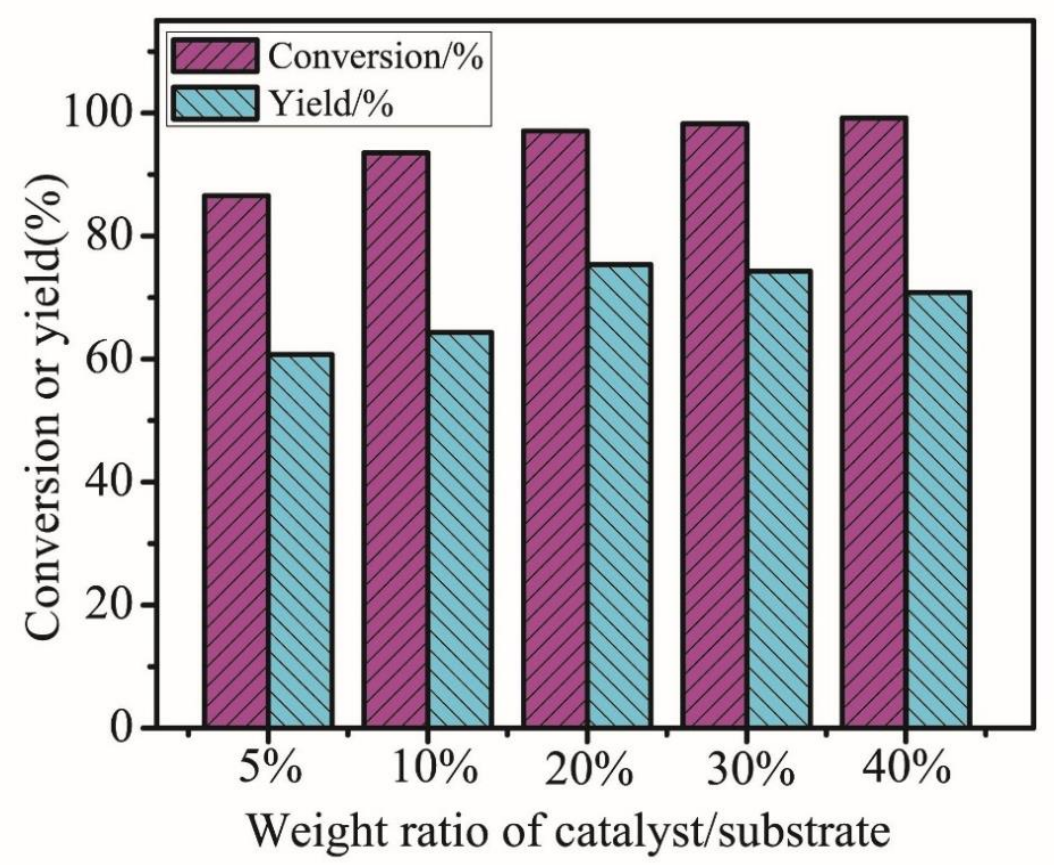

Fig.4.6 The influence of ratio of catalyst/substrate on the conversion and HMF selectivity. Reaction conditions: fructose $(500 \mathrm{mg}$ ), catalyst (following the ratio), DMSO (10 mL), $140{ }^{\circ} \mathrm{C}$ for $0.5 \mathrm{~h}$.

\subsubsection{Effect of temperature}

The reaction is carried out at different temperatures from 100 to $160{ }^{\circ} \mathrm{C}$ and the results are summarized in Fig. 4.7. The conversion of fructose is only $12.8 \%$ at $100{ }^{\circ} \mathrm{C}$ after $0.5 \mathrm{~h}$. The conversion reaches $100 \%$ within the same reaction time duration at $160{ }^{\circ} \mathrm{C}$. The selectivity of $\mathrm{HMF}$ also increases from 40.5 to $76.3 \%$ as the temperature increases from 100 to $160{ }^{\circ} \mathrm{C}$. When the temperature is higher than $140{ }^{\circ} \mathrm{C}$, the reaction finishes $(100 \%$ conversion) in $1.5 \mathrm{~h}$. Longer reaction time leads to the slight decrease in HMF yield because HMF is unstable under high temperatures [386]. 


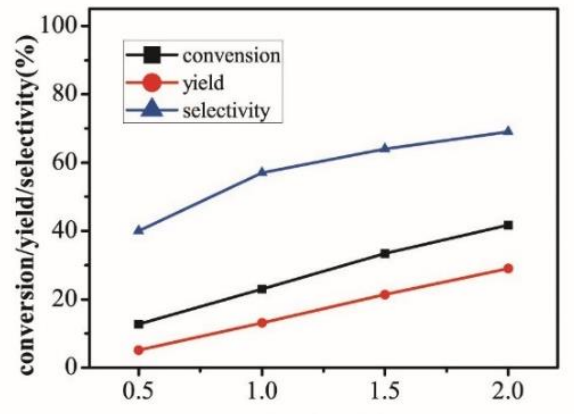

(a)Time (h)

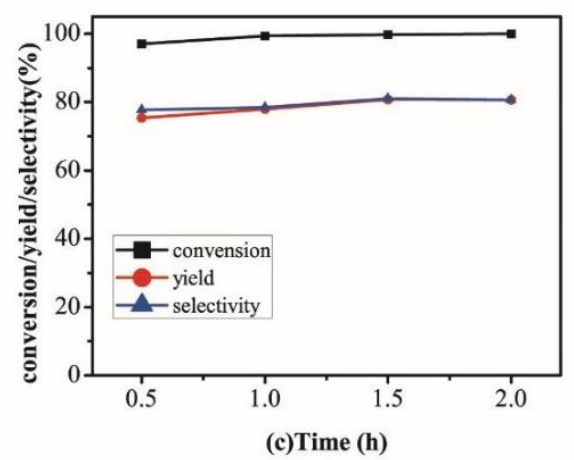

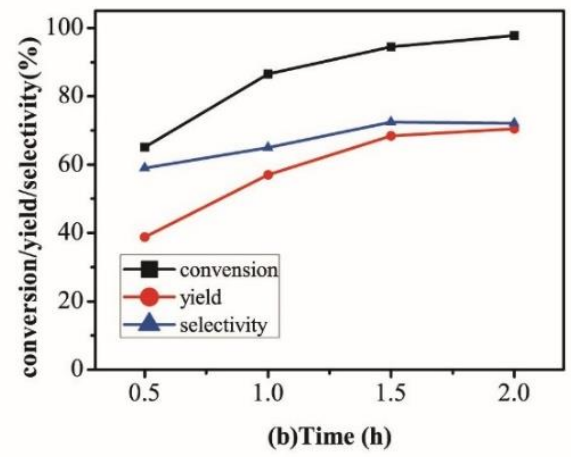

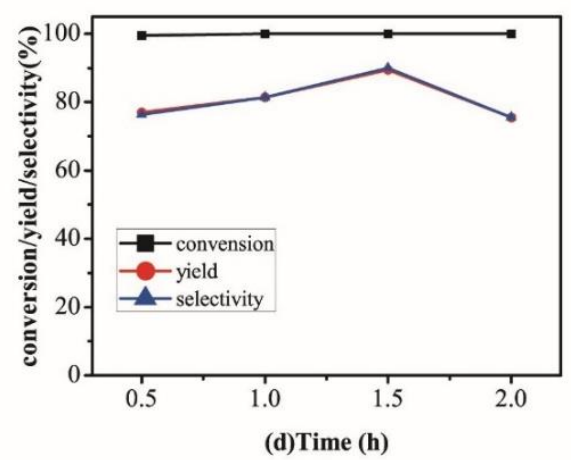

Fig.4.7 Influence of reaction time and temperature on the dehydration of fructose to HMF. Reaction conditions: fructose $(500 \mathrm{mg})$, catalyst(100 mg), $\operatorname{DMSO}(10 \mathrm{~mL}),(\mathrm{a}) 100{ }^{\circ} \mathrm{C}$, (b) $120^{\circ} \mathrm{C}$, (c) $140^{\circ} \mathrm{C}, \quad$ (d) $160{ }^{\circ} \mathrm{C}$.

\subsubsection{Effect of reaction time}

The effect of reaction time duration on the reaction is also investigated and the results are shown in Fig. 4.7. The fructose conversion, HMF selectivity and yield keep increasing along with the reaction time as far as the reaction temperature is below $140{ }^{\circ} \mathrm{C}$ though there is a little decrease in the growth rate. When conducting the reaction at the temperature higher than $140{ }^{\circ} \mathrm{C}$, the conversion of fructose quickly reaches almost $100 \%$, indicating the superior catalytic activity, while the selectivity of HMF firstly increases then decreases, e.g., Fig. 7(d) shows the reaction conducted at $160{ }^{\circ} \mathrm{C}$, the $\mathrm{HMF}$ selectivity increases from $76.3 \%(0.5 \mathrm{~h})$ to $90.1 \%(1.5 \mathrm{~h})$ then decreases to $75.6 \%(2 \mathrm{~h})$. This decrease in selectivity suggests that the long reaction time leads to the further condensation of HMF into byproducts especially at high reaction temperature $[387,388]$.

\subsection{The adsorption properties test}


The adsorption of fructose and HMF by CS is studied in DMSO solution at room temperature. A certain amount of CS is added into 5ml DMSO solution containing $480 \mathrm{mg}$ of fructose or $100 \mathrm{mg}$ of HMF. The suspension is filtered and analyzed by HPLC after stirring for $6 \mathrm{~h}$ under room temperature. As shown in Table 4.4, the catalyst adsorbs fructose in the solution but hardly adsorbs HMF. The adsorption amount of fructose is remarkably higher compared with Amberlyst-15 [229]. The strong adsorption of reactant and quick desorption of product play an important role in affording the high catalytic activity and good selectivity toward desired product.

Table 4.4 The adsorption properties of fructose or HMF by CS

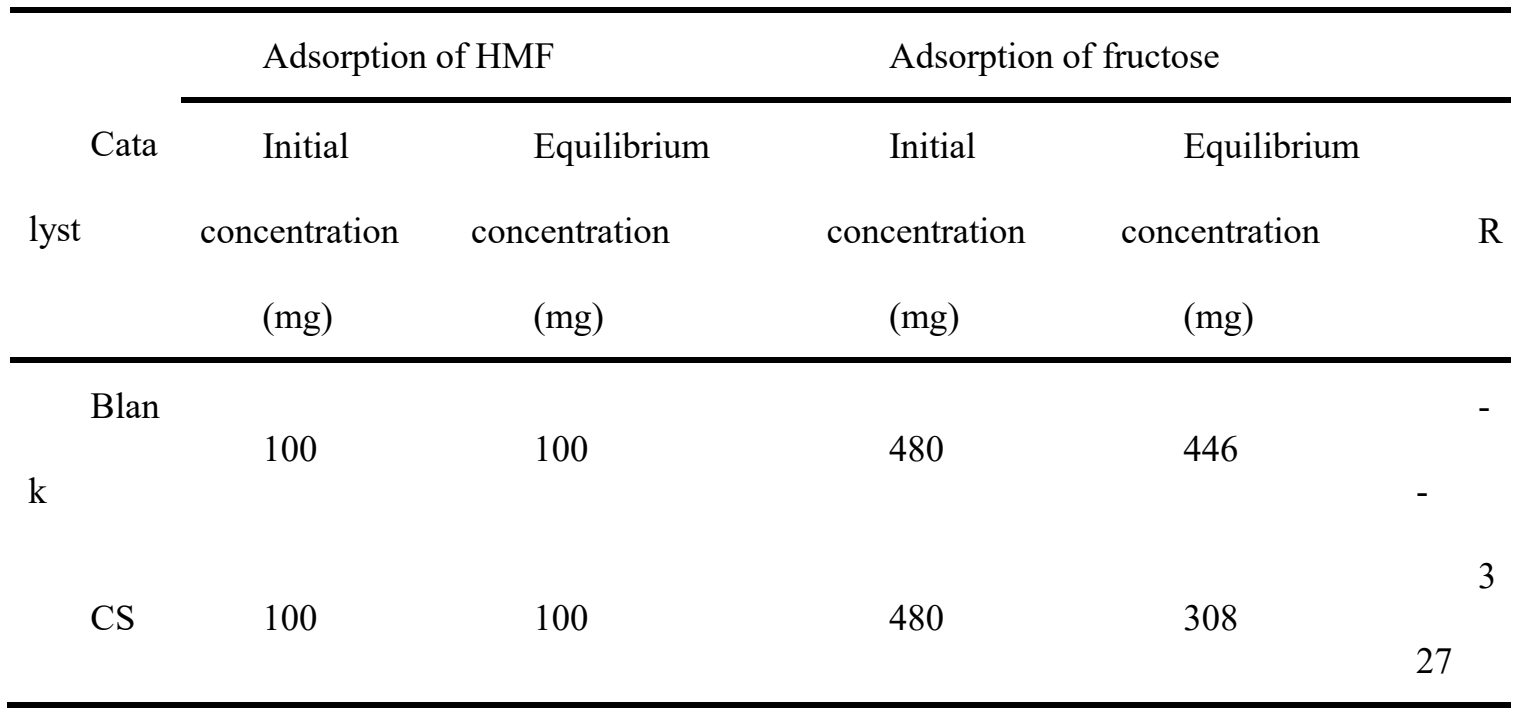

$\mathrm{R}$ represents the adsorption quantity of HMF or fructose per gram of catalyst $(\mathrm{mg} / \mathrm{g})$

\subsection{Reusability of the catalyst}

The results of stability and reusability of the catalyst are show in Fig. 4.8. After each reaction run, the catalyst is separated from the reaction mixture and washed with hot water and ethanol, then dried under vacuum at $80{ }^{\circ} \mathrm{C}$ for $4 \mathrm{~h}$. There is a slight decrease in both fructose conversion and HMF selectivity after the first run. The conversion and selectivity remain constant during the following cycles. Fructose conversion of $96.2 \%$ and HMF selectivity of $73.9 \%$ can be maintained even after the $5^{\text {th }}$ run, indicating a good recyclability of the catalyst. 
To further study the stability of $-\mathrm{SO}_{3} \mathrm{H}$ groups, a leaching test was conducted. $100 \mathrm{mg}$ catalyst was dissolved in $10 \mathrm{~mL}$ DMSO, heated at $140{ }^{\circ} \mathrm{C}$ and stirring for $2 \mathrm{~h}$. Then the solution was filtered and the filtrate was used to conduct the dehydration experiment. Results showed in Fig. S1 that the fructose conversion and HMF yield was similar to that of a blank experiment, indicating the high stability of $-\mathrm{SO}_{3} \mathrm{H}$. It was confirmed by element analysis in Table $\mathrm{S} 1$ that the $\mathrm{S}$ content in the catalyst was almost constant. In addition, Fig. S2 showed that the morphology of the catalyst didn't change after the reaction indicating its fairly stable structure.

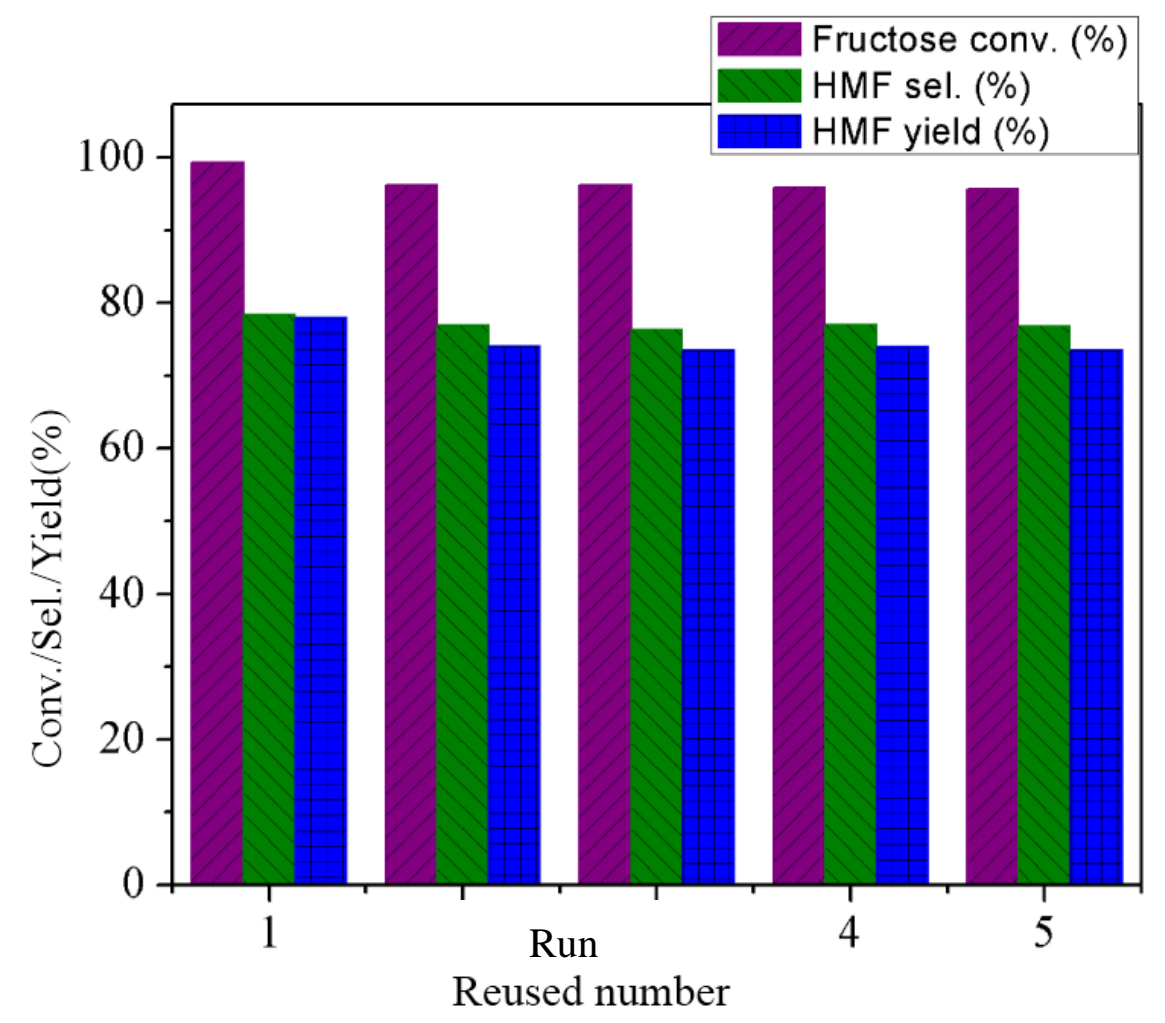

Fig.4.8 Fructose conversions and selectivity towards HMF in the recycling runs. Reaction conditions: fructose (500 mg), catalyst (100 mg), DMSO (10 mL), $140{ }^{\circ} \mathrm{C}, 1 \mathrm{~h}$

\subsection{Discussion}

The dehydration of fructose to HMF is an acid-catalyzed reaction consisting of several steps such as protonation, dehydration and deprotonation. Sulfonated 
CS catalyst, as a strong protonic acid, is suitable for the conversion of fructose to HMF and usually a higher acid density affords better catalytic performances. Carbon-based solid acids are commonly prepared by the sulfonation of different carbon precursors. Konwar et al. [203] studied the influence of different carbon precursors in the preparation of carbon-based solid acid and found that highly carbonized materials were difficult to be sulfonated. In this study, the CSs prepared from the aromatization and carbonization of glucose under hydrothermal conditions were incomplete carbonation materials [389], and they were easy to be sulfonated with high density $-\mathrm{SO}_{3} \mathrm{H}$ groups on surfaces, showing superior catalytic performances. Different from the CSs prepared by one-step method, the size and morphology of CSs prepared by the two-step method did not change with the concentrations of sulfuric acid solutions, indicating its stable structure and possibility of tuning the acid density on surfaces without changing the carbon-based support. Furthermore, the large surface areas due to the low degree of agglomeration afforded surfaces for $-\mathrm{SO}_{3} \mathrm{H}$ groups grafting in the sulfonation step and enhanced the contact between fructose and $-\mathrm{SO}_{3} \mathrm{H}$ groups in the reaction, which contributed to the high catalytic efficiency. Moreover, the strong fructose adsorption and quick HMF desorption capabilities of the CS contributed to the catalytic performances.

In addition to the catalyst, selection of solvent also played an important role in fructose dehydration. Different reaction pathways existed in the dehydration of fructose to HMF in the presence of DMSO solvent. Amarasekara et al. [381] demonstrated that DMSO catalyzed the dehydration of fructose to HMF due to the $\mathrm{S}=\mathrm{O}$ bond in DMSO that acted as the catalyst. Similarly, the $\mathrm{S}=\mathrm{O}$ structure in the $-\mathrm{SO}_{3} \mathrm{H}$ groups on $\mathrm{CS}$ solid acid surfaces can play the similar role. Therefore, in the presence of CS solid acid, both protonic acid-catalysis (Bronsted acid catalysis) and $\mathrm{S}=\mathrm{O}$-catalysis synergistically worked in the reaction system. In addition, the DMSO solvent prevented fructose from side reactions such as the 
rehydration of HMF to levulinic acid, formic acid and humins because the cleavage of $\mathrm{C} 1$ carbon in HMF led to the hydration of HMF to levulinic acid and formic acid. DMSO preferred stay in coordination with the hydroxyl group and $\mathrm{C} 1$ carbon in HMF, which effectively provided a shielding effect to the HMF molecule [390].

\subsection{Summary}

In summary, a sulfonated CS solid acid catalyst bearing $-\mathrm{SO}_{3} \mathrm{H},-\mathrm{OH}$ and $\mathrm{COOH}$ groups was synthesized by a two-step hydrothermal method. Aqueous sulfuric acid solution replaced the concentrated or fumed sulfuric in the sulfonation step. The acid density and BET surface area of these CS solid acid catalysts increased with the concentrations of the sulfonation solution. The catalyst showed good performances in the dehydration of fructose to HMF under mild conditions, e.g., $100 \%$ fructose conversion can be obtained in $0.5 \mathrm{~h}$ and $\mathrm{HMF}$ yield can reach $90 \%$ in $1.5 \mathrm{~h}$ at $160^{\circ} \mathrm{C}$. High acid density and reaction temperature accelerated the reaction and improved the selectivity. Nonetheless, excessive amounts of acid sites and high temperature resulted in slight decrease in HMF selectivity due to the further condensation of HMF into byproducts. Besides, high purity HMF can be obtained through extraction by tetrahydrofuran[391](Fig. S3) and there was no significant loss in activity after five catalytic cycles, indicating the high stability and reusability of the catalyst.

This work has been published on Chemical Society Reviews (Catalysis Today, Volume 264, 15 April 2016, Pages 123-130), adopted in this chapter with the permission of Royal Society of Chemistry. 
Appendix: Supporting Information

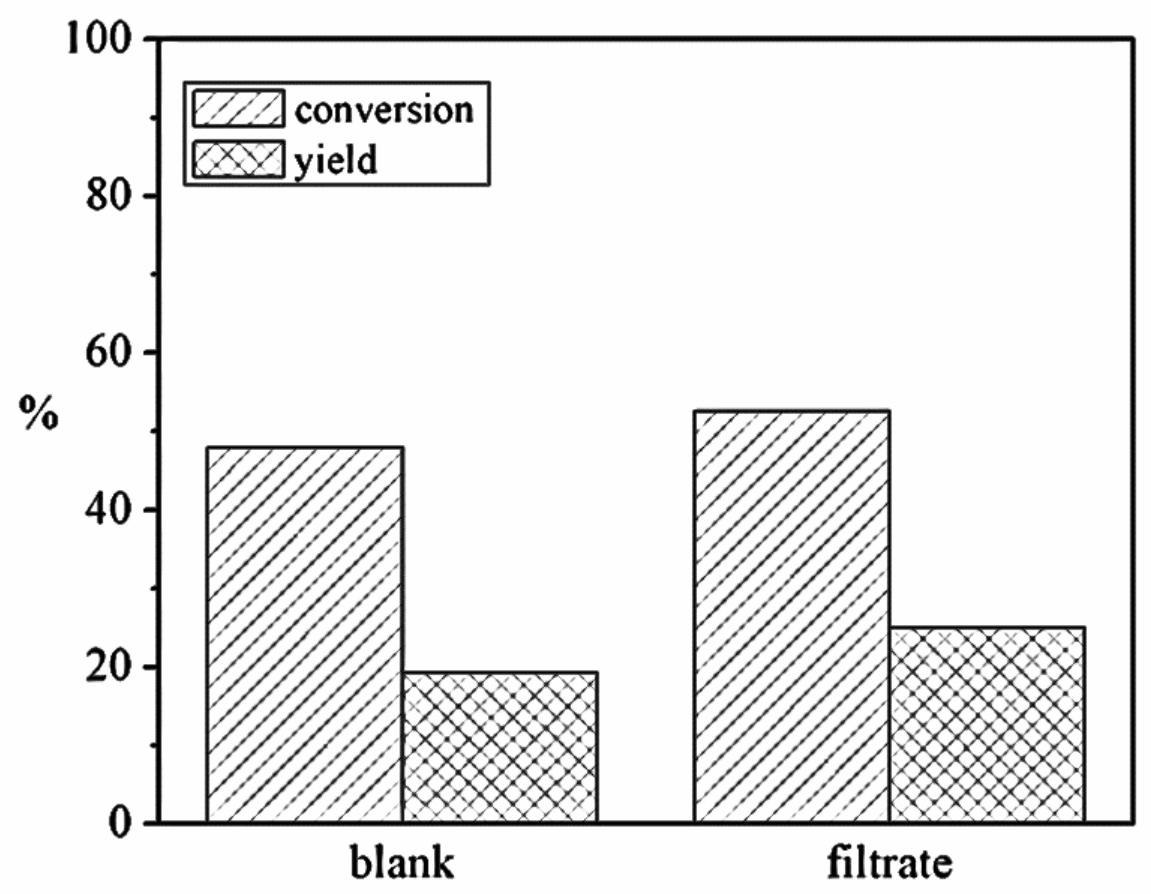

Fig. S1. Leaching test of sulfonic acid groups on CS acid.

Table S1 Element analysis of CS catalyst before and after reaction.

\begin{tabular}{lllll}
\hline Samples & $\mathrm{C} \%$ & $\mathrm{H} \%$ & $\mathrm{O} \%$ & $\mathrm{~S} \%$ \\
\hline $\mathrm{CS}-2$ & 63.00 & 3.87 & 32.52 & 0.55 \\
$\mathrm{CS}^{2} 2^{\mathrm{a}}$ & 61.75 & 4.09 & 33.63 & 0.53 \\
\hline
\end{tabular}

${ }^{a}$ the catalyst was recycled after reaction.

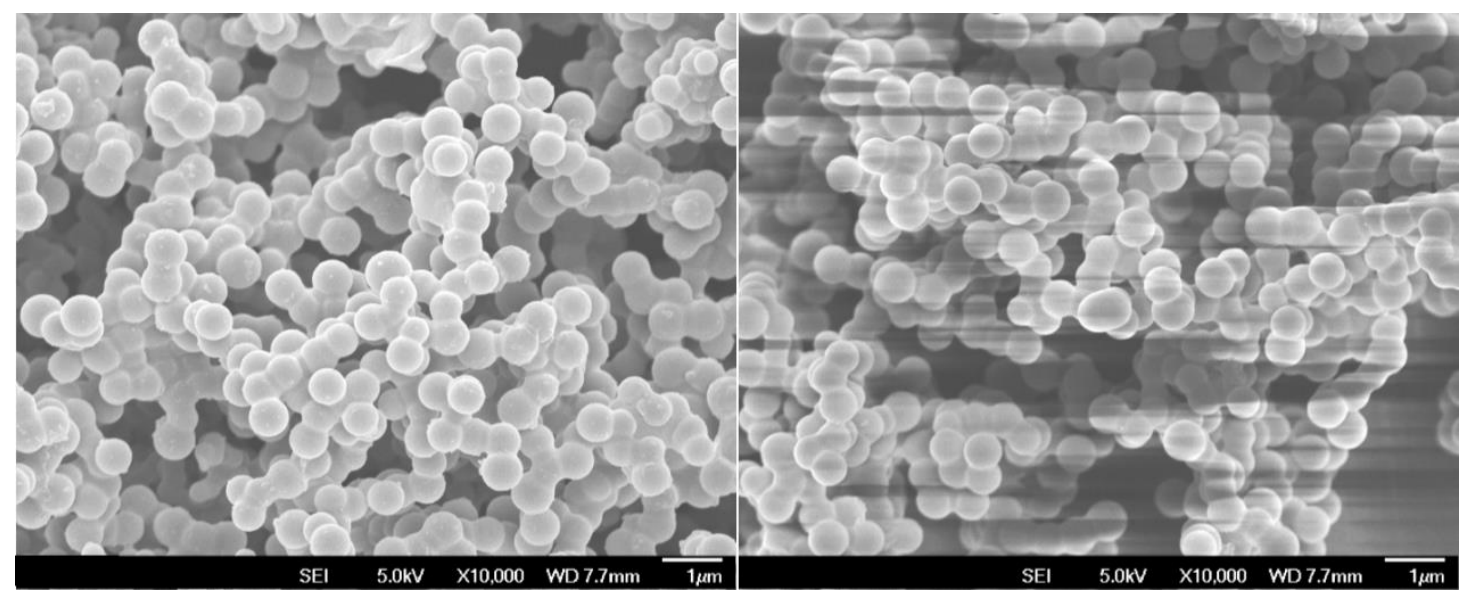

Fig. S2. FESEM pictures of CS-2 before and after reaction. 


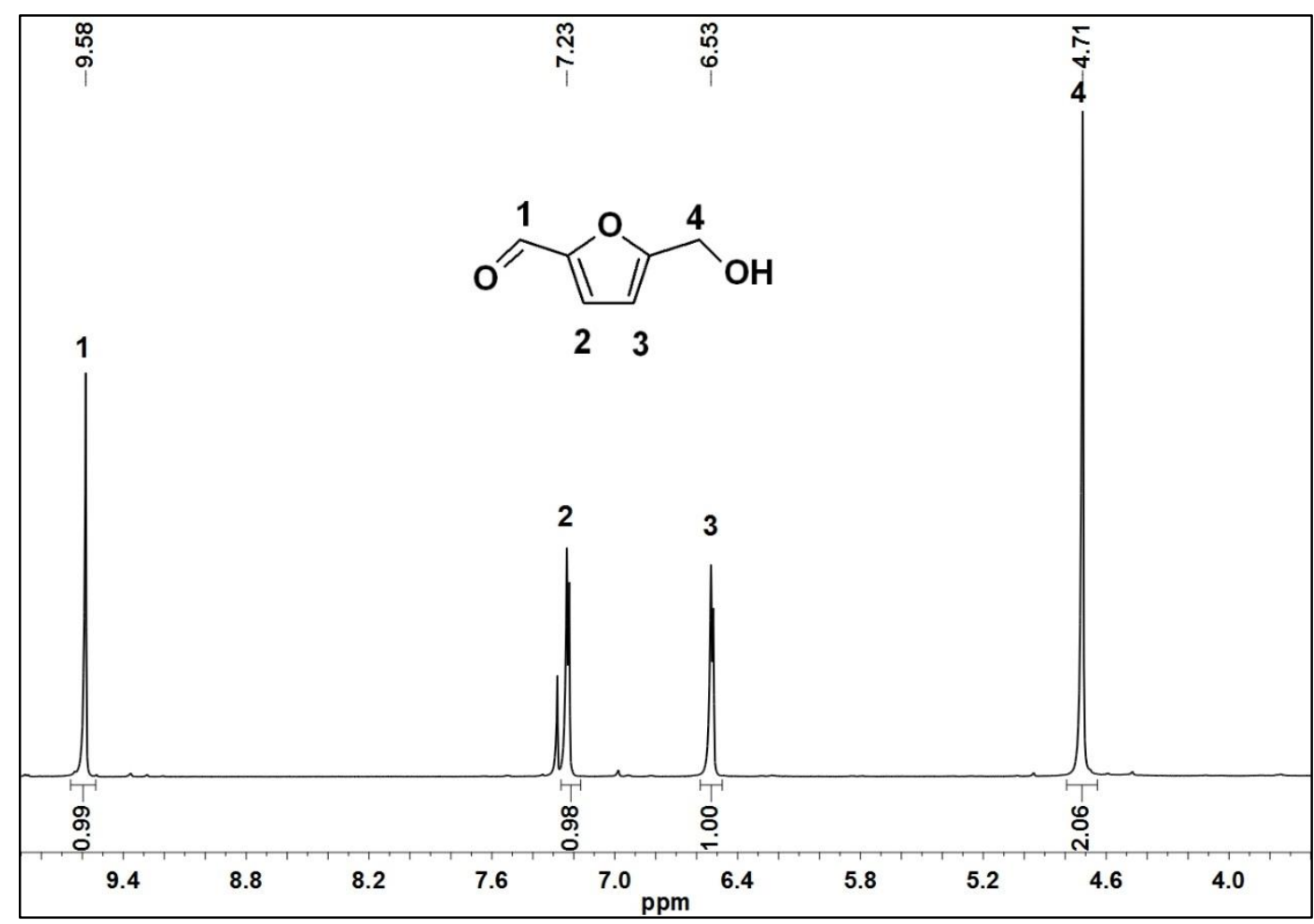

Fig. S3. ${ }^{1} \mathrm{H}-\mathrm{NMR}$ spectra of HMF isolated from the reaction mixture.

${ }^{1} \mathrm{H}-\mathrm{NMR}$ for $\mathrm{HMF}\left(300 \mathrm{MHz}, \mathrm{CDCl}_{3}\right): \delta 9.58(\mathrm{~s}, 1 \mathrm{H}, \mathrm{CHO}), \delta 7.23(\mathrm{~d}, 1 \mathrm{H}$, furan ring $\mathrm{CH}), \delta 6.53(\mathrm{~d}, 1 \mathrm{H}$, furan $\operatorname{ring} \mathrm{CH}), \delta 4.71\left(\mathrm{~s}, 2 \mathrm{H}, \mathrm{CH}_{2}\right)$. 
Chapter 5 Selective Aerobic Oxidation of 5-hydroxymethyl-2-furfural into 2, 5-diformylfuran over V-embedded Mesoporous Carbon Microspheres

In this chapter, V-embedded mesoporous carbon microspheres were synthesized an applied in the aerobic oxidation of HMF to DFF. The catalytic performance of the catalysts prepared under different conditions were studied and the changes in the morphology of the catalysts were studied with characterization methods such as XRD, SEM, BET, FTIR, Raman spectroscopy and elemental analysis. The catalysts showed high activity in the oxidation of HMF to DFF. Under optimized reaction conditions, HMF conversion of $100 \%$ and DFF yield of more than $99 \%$ were obtained in DMSO at $120^{\circ} \mathrm{C}$ with oxygen bubbling at a flow rate of $20 \mathrm{~mL} / \mathrm{min}$ after $9 \mathrm{~h}$.

5.1 Catalyst characterization of V-embedded mesoporous carbon microspheres 


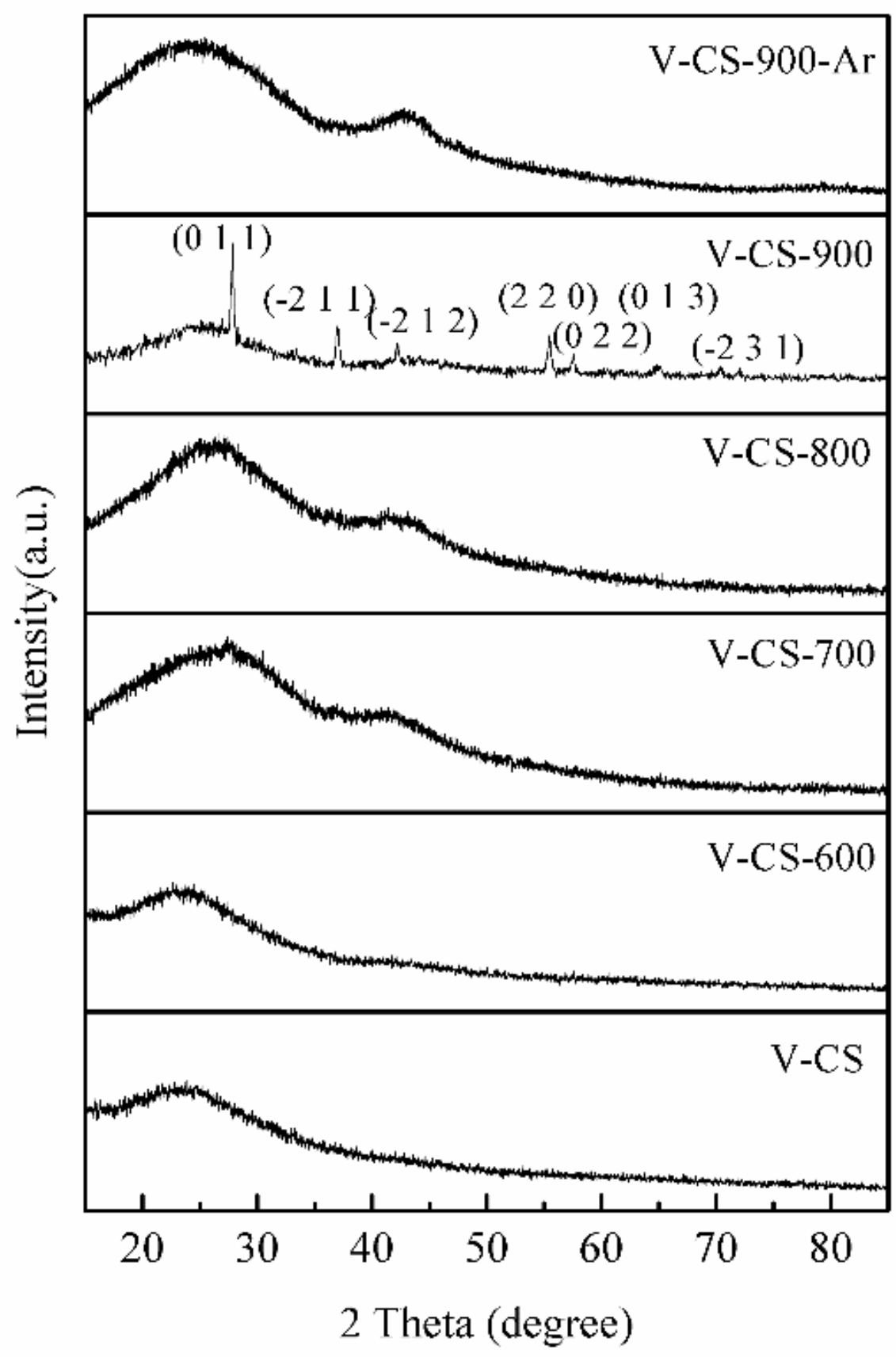

Fig. 5.1 XRD analysis of V-CS before and after thermal treatment

In Fig.5.1, the XRD patterns of the as prepared catalysts exhibit a broad peak in the 2 theta range $10-30^{\circ}$ which was attributed to $\mathrm{C}\left(\begin{array}{lll}0 & 0 & 2\end{array}\right)$ interlayer reflection among irregularly oriented polycyclic aromatic carbon sheets[392]. This peak shifts toward higher degrees and become relatively sharper after calcination, indicating a decrease in the interlayer space[393]. Another broad peak in the 2 theta range $35-50^{\circ}$ corresponding to the $\mathrm{C}\left(\begin{array}{lll}1 & 0 & 0\end{array}\right)$ diffraction peak becomes more 
evident with increasing calcination temperature, which is a sign of the increasing degree of ordered structure in the catalysts[394]. These two broad peaks suggest that the carbon material of the catalysts are amorphous. No other peaks exist for the catalysts except V-CS-900, indicating the homogeneous distribution of vanadium in the carbon spheres. The typical diffraction peaks of V-CS-900 can be perfectly indexed to the VO2 (M)[395], demonstrating that the aggregation of the highly dispersed VO2 (M) in the carbon spheres occurred during the calcination process because of the high temperature and the decomposition of the unstable carbon layer.

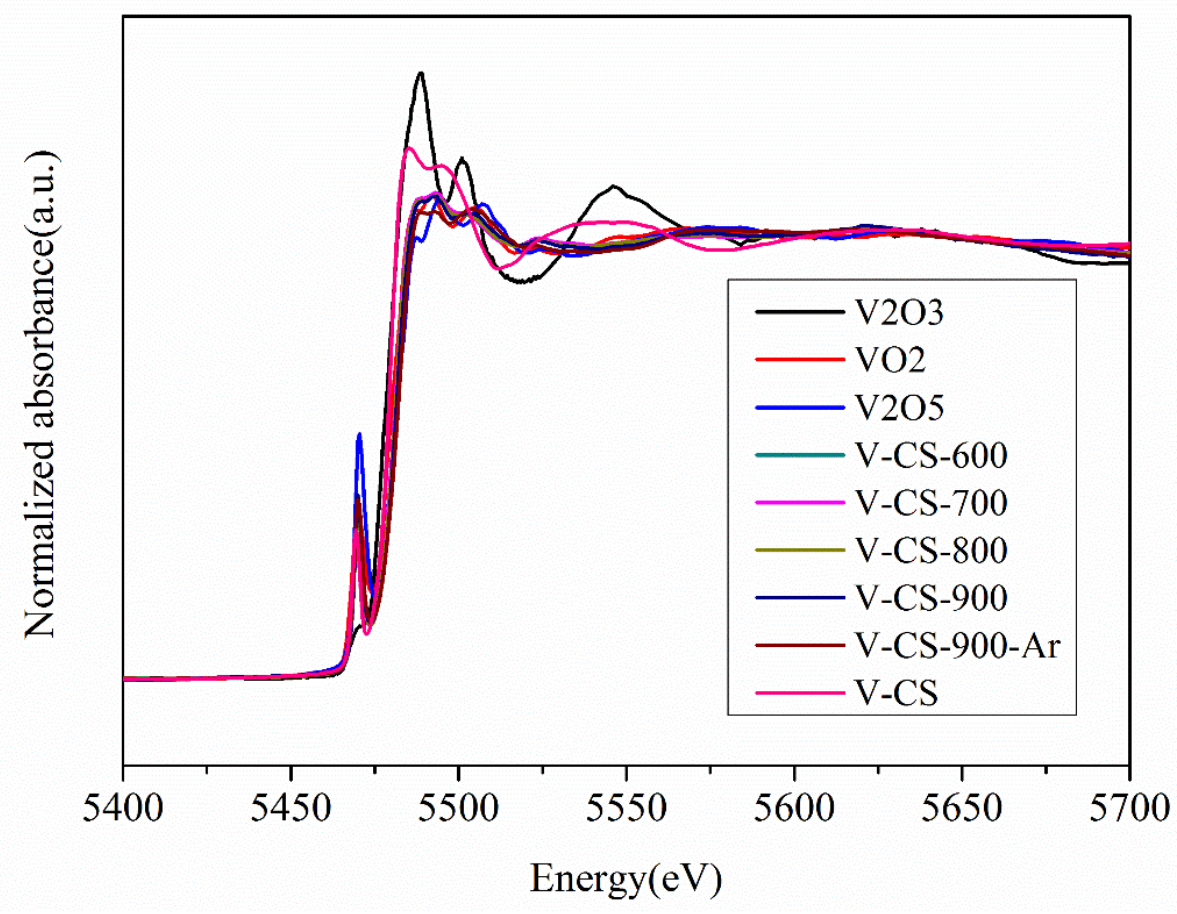

Fig. 5.2 XANES spectra of V-CS before and after thermal treatment

Since XRD was not able to provide enough information on $\mathrm{V}$ because of its low loading and low aggregation state, XANES measurements of these catalysts were performed to characterize the coordination and oxidation state of the vanadium species. Also reference substances with different vanadium coordination and oxidation states were measured. It can be seen that there is an obvious change in the XANES spectra for the catalysts before and after calcination in Fig.5.2. The spectra of V-CS calcined at $600^{\circ} \mathrm{C}, 700^{\circ} \mathrm{C}, 800^{\circ} \mathrm{C}$ and 
$900^{\circ} \mathrm{C}$ are comparable, indicating a similar local environment of V. Due to the high sensibility of the XANES region to the geometric structure and the specific bonding situation around the absorber atom it is concluded that the $\mathrm{VO}_{\mathrm{x}}$ embedded in the carbon sphere after calcination is similar to $\mathrm{VO}_{2}$, suggesting that the calcination can promote the $\mathrm{VO}_{\mathrm{x}}$ species evolve to form $\mathrm{VO}_{2}$ [396]. It is worth noting that the oscillation magnitudes of the samples are weaker than $\mathrm{VO}_{2}$ because the samples are amorphous state.

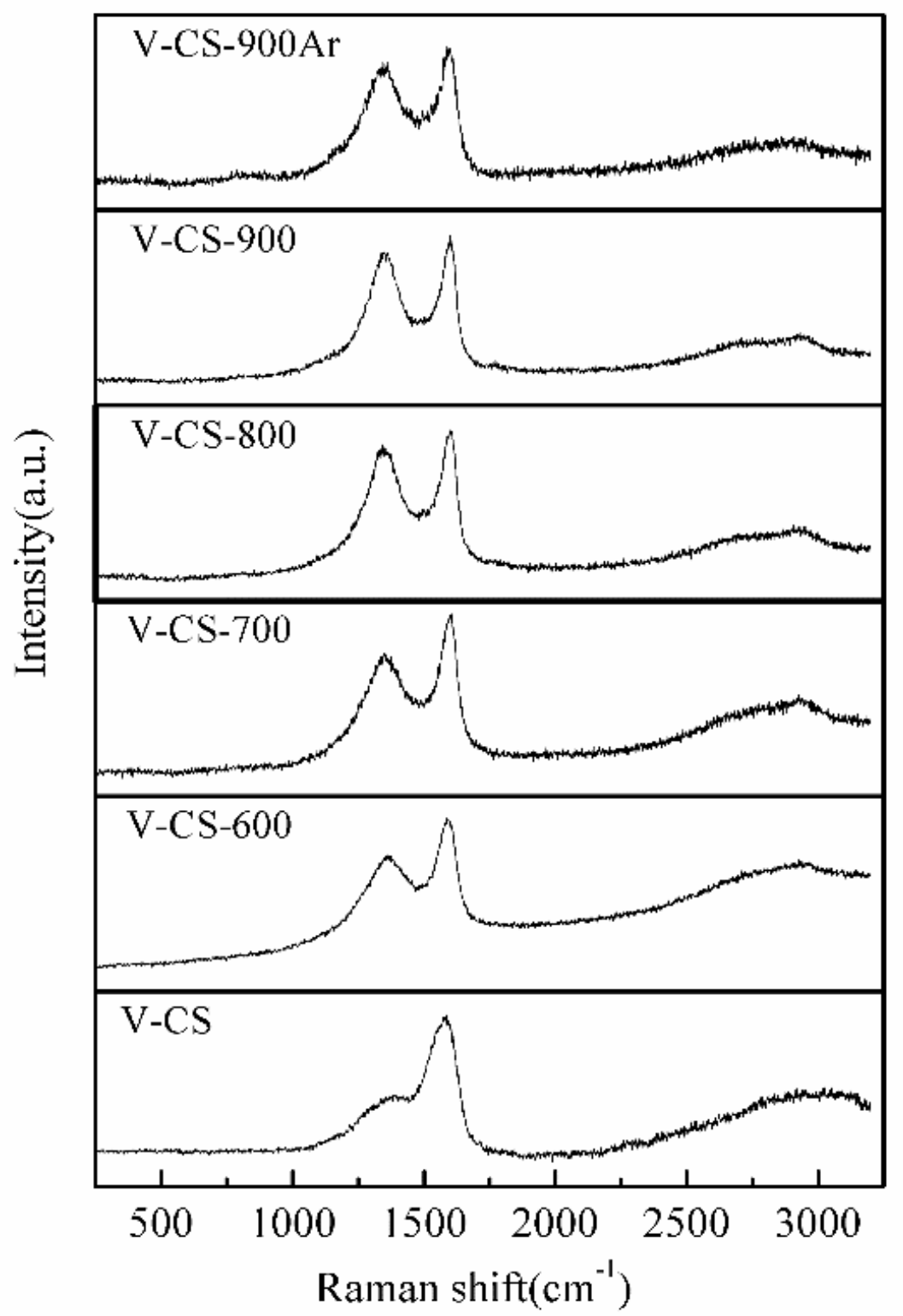

Fig.5.3 Raman spectra of V-CS before and after thermal treatment 
Fig.5.3 shows the Raman spectra of the as-prepared catalysts. Tow typical peaks attributed to the $\mathrm{D}$ and $\mathrm{G}$ bands can be seen for each catalyst. The peaks at around $1340 \mathrm{~cm}-1$ is associated to the disordered structure of the carbon microspheres while the peaks at $-1600 \mathrm{~cm}-1$ corresponds to the presence of crystalline graphitic carbon. The $\mathrm{I}_{\mathrm{D}} / \mathrm{I}_{\mathrm{G}}$ values of the catalysts are all larger than 0.5 , indicating that the amorphous carbon structure has a high content of defects in the graphite sheets[393]. The value of $I_{D} / I_{G}$ for $V-C S$ is 0.58 and this value increase dramatically to 1.41 after calcination at $600^{\circ} \mathrm{C}$. And, the $\mathrm{I}_{\mathrm{D}} / \mathrm{I}_{\mathrm{G}}$ ratios increase from 1.41 to 1.86 as the calcination temperature increase from $600^{\circ} \mathrm{C}$ to $900^{\circ} \mathrm{C}$, which can be attributed to the change of the microstructure of carbon sheets in carbon spheres. Though calcined at same temperature, the $\mathrm{I}_{\mathrm{D}} / \mathrm{I}_{\mathrm{G}}$ of $\mathrm{V}$ CS-900-Ar is lower than V-CS-900 because of the different atmosphere during the calcination.

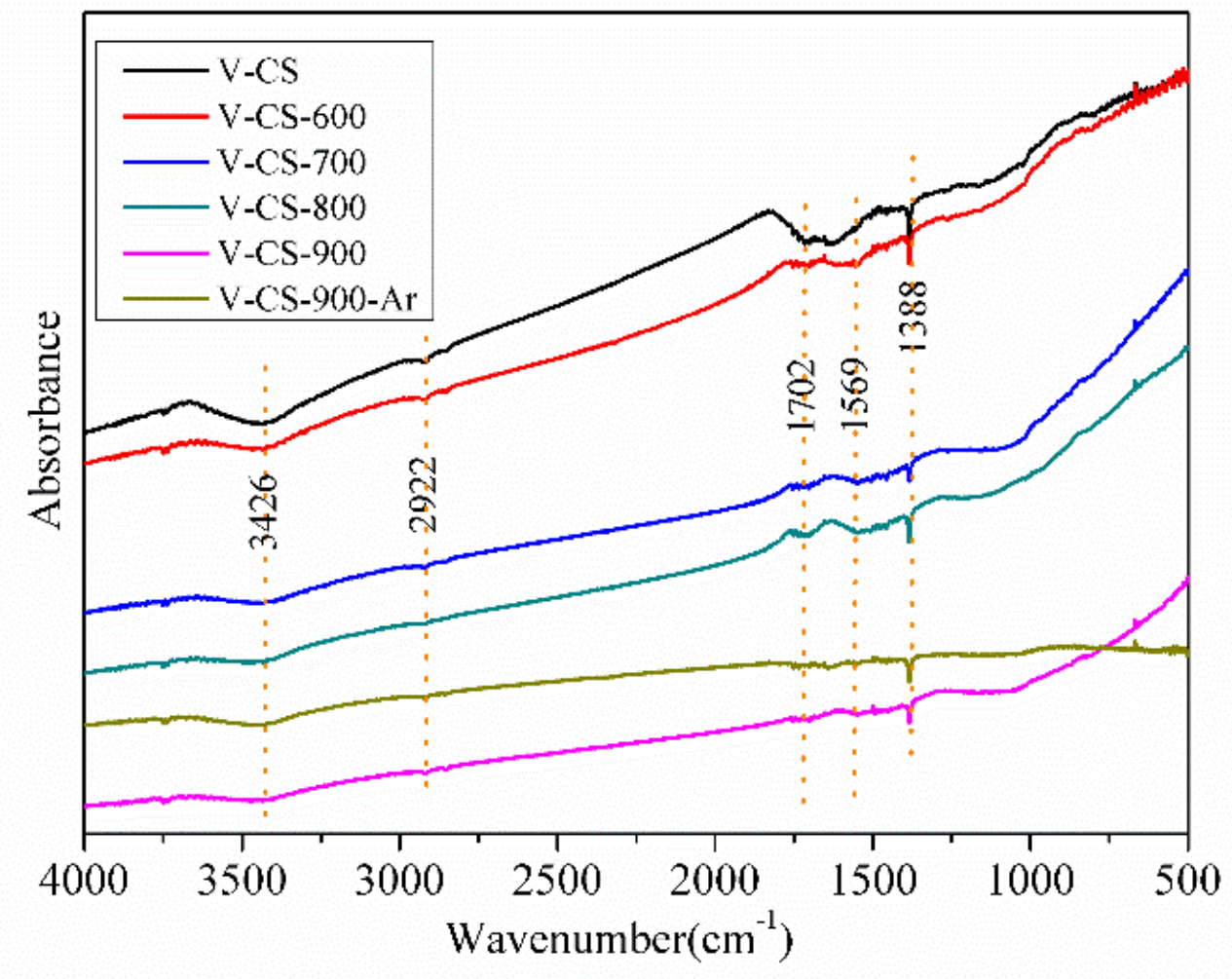

Fig. 5.4 FTIR spectra of V-CS before and after thermal treatment 
FTIR spectroscopy was utilized to study V-embedded mesoporous carbon microspheres. In Fig. 5.4, the broad band around $3426 \mathrm{~cm}^{-1}$ is attributed $\mathrm{O}-\mathrm{H}$ stretching vibration. The peaks at 2922 and $1388 \mathrm{~cm}^{-1}$ can be assigned to stretching and bending mode of $\mathrm{C}-\mathrm{H}$ bond. The $\mathrm{C}=\mathrm{O}$ vibration of aldehydes, ketones, carboxylic acids or esters and the $\mathrm{C}=\mathrm{C}$ bond of alkenes or aromatic rings appears at $1702 \mathrm{~cm}^{-1}$ and around $1569 \mathrm{~cm}^{-1}$ respectively[395].

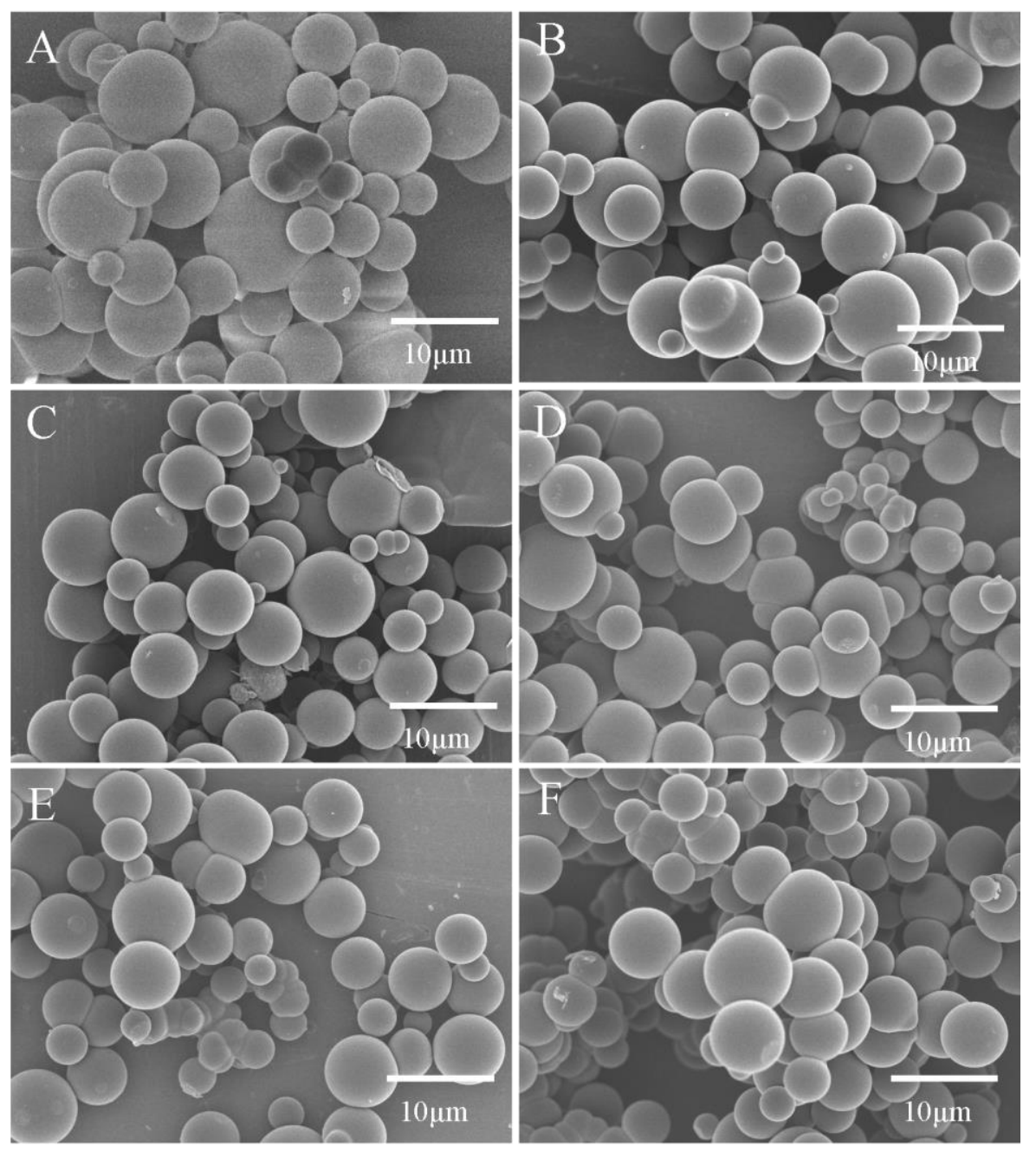

Fig.5.5 FESEM images of (A) V-CS, (B) V-CS-600, (C) V-CS-700, (D) VCS-800, (E) V-CS-900, (F) V-CS-900-Ar

Fig.5.5 shows general morphology of the as-synthesized catalysts. As can be seen, these carbon spheres are almost monodispersed and the particle size ranges from 3 to 10 um (Fig. 5.5 A). After the calcination, the spherical morphology remains unchanged. The size of the vanadium-embeded carbon microspheres 
does not reduce significantly, indicating the high stability of the carbon structure even at temperature up to $900^{\circ} \mathrm{C}$.

Table 5.1. Elemental composition of V-CS before and after thermal treatment

\begin{tabular}{llllll}
\hline Catalyst & Carbon $^{\mathrm{a}}$ & Hydrogen $^{\mathrm{a}}$ & Oxygen $^{\mathrm{a}}$ & Nitrogen $^{\mathrm{a}}$ & Vanadium $^{\mathrm{b}}$ \\
\hline V-CS & 56 & 4.09 & 38.17 & 0.79 & 0.95 \\
V-CS-600 & 67.1 & 2.83 & 27.39 & 1.15 & 1.53 \\
V-CS-700 & 67.55 & 2.57 & 25.74 & 1.1 & 3.04 \\
V-CS-800 & 56.66 & 2.47 & 36.41 & 0.98 & 3.48 \\
V-CS-900 & 61.28 & 2.44 & 30.66 & 0.5 & 5.12 \\
V-CS-900-Ar & 79.94 & 1.65 & 16.17 & 0.41 & 1.83 \\
\hline
\end{tabular}

${ }^{\text {a }}$ Determined by elemental analysis

${ }^{\mathrm{b}}$ Determined by TGA[397]

Table 5.1 lists the chemical composition of the as-synthesized catalysts. The carbon content of V-CS is $56 \%$, this value increases significantly after the calcination due to the decomposition of the carbon sphere. Comparing with V$\mathrm{CS}$, the hydrogen and oxygen content decreases dramatically as a result of the falling of groups such as hydroxyl, aldehyde and carboxyl group at high calcination temperature. The vanadium content is determined by thermogravimetric analysis under air atmosphere. It can be seen that the vanadium content increases along with the rising of the calcination temperature. The vanadium content increases from $0.95 \%$ ( V-CS) to $5.12 \%$ ( V-CS-900), which is caused by the loss of carbon during the calcination by the reaction 
between the carbon atoms or fragments and oxygen in limited air atmosphere. This explanation can also be confirmed by the comparison of vanadium content between V-CS-900 and V-CS-900-Ar, the vanadium content of V-CS-900-Ar is much lower than V-CS-900 since there is no oxygen to react with carbon atoms to reduce the carbon amount under argon atmosphere.

Table 5.2. Textual properties of V-CS before and after thermal treatment

\begin{tabular}{llllll}
\hline Catalyst & $\begin{array}{l}\text { S } \\
\left(\mathrm{m}^{2} / \mathrm{g}\right)\end{array}$ & $\begin{array}{l}\mathrm{V}_{\text {micro }} \\
\left(\mathrm{m}^{3} / \mathrm{g}\right)\end{array}$ & $\begin{array}{l}\mathrm{V}_{\text {macro\&meso }} \\
\left(\mathrm{m}^{3} / \mathrm{g}\right)\end{array}$ & $\begin{array}{c}\mathrm{V}_{\text {total }} \\
\left(\mathrm{m}^{3} / \mathrm{g}\right)\end{array}$ & $\begin{array}{c}\mathrm{D} \\
(\AA)\end{array}$ \\
\hline V-CS & 60 & - & - & - & - \\
V-CS-600 & 559 & 0.189 & 0.081 & 0.27 & 19.3 \\
V-CS-700 & 613 & 0.208 & 0.089 & 0.297 & 19.4 \\
V-CS-800 & 765 & 0.23 & 0.16 & 0.39 & 20.4 \\
V-CS-900 & 934 & 0.252 & 0.276 & 0.528 & 22.6 \\
V-CS-900-Ar & 515 & 0.197 & 0.047 & 0.244 & 18.9 \\
\hline
\end{tabular}

In order to investigate the physical properties of the as-synthesized catalysts, the samples were further characterized by BET. Shown in Table 5.2, the BET surface area for V-CS is very low, and no significant porosity is detected. However, the BET surface area increases from $60 \mathrm{~m}^{2} / \mathrm{g}$ to $550 \mathrm{~m}^{2} / \mathrm{g}$ after calcination at $600^{\circ} \mathrm{C}$ in air and up to $936 \mathrm{~m}^{2} / \mathrm{g}$ at $900^{\circ} \mathrm{C}$. In addition, the pore volume and pore size increase from $0.27 \mathrm{~m}^{3} / \mathrm{g}$ and $19.3 \AA$ to $0.528 \mathrm{~m}^{3} / \mathrm{g}$ and 22.6 $\AA$ respectively as the calcination temperature raise from $600^{\circ} \mathrm{C}$ to $900^{\circ} \mathrm{C}$. It deserved to note that the pore volume and BET surface area of V-CS-900-Ar are even smaller than V-CS-600 though the latter is calcined at a $300^{\circ} \mathrm{C}$ lower temperature. Therefore, the oxygen plays an important role in making pores during the calcination process, which is consistent with the results of element analysis.

\subsection{Catalytic performance of catalysts}

\subsubsection{Catalytic oxidation of HMF over V-embedded carbon spheres}


The catalytic activity of the as-prepared catalysts was evaluated by the oxidation of HMF in DMSO by bubbling oxygen under atmospheric pressure at $120^{\circ} \mathrm{C}$. Since $\mathrm{VO}_{2}$ was supposed to be the active site and its weight percent was different for every catalyst, the dosage of each catalyst was different in order to ensure the amount of VO2 is equal in the reaction system. Fig.5.6 shows the results of $\mathrm{HMF}$ oxidation using various catalysts. Low HMF conversion of $0.77 \%$ was obtained when using V-CS as the catalyst. After calcination at $600-900{ }^{\circ} \mathrm{C}$, the activity of the catalyst increase significantly. It can be seen that the HMF conversion of $34.13 \%$ and DFF yield of $30.93 \%$ can be observed in the presence of V-CS-600. In addition, the HMF conversion and DFF yield increased with the enhancement of the calcination temperature. These results demonstrate that the $\mathrm{VO}_{2}$ was encapsulated by the carbon sphere, leading to few contact chance between the reactants and active sites when V-CS was used as catalyst. For the catalysts such as V-CS-600, V-CS-700 and V-CS-800, the porous structure and high interface improved the contact efficiency between $\mathrm{HMF}$ and $\mathrm{VO}_{2}$. Thus the HMF conversion and DFF yield increase from $34.13 \%$ and $30.93 \%$ to $63.00 \%$ and $62.95 \%$ respectively as the calcination temperature raise from $600^{\circ} \mathrm{C}$ to $800^{\circ} \mathrm{C}$ resulting in increasing surface area and pore volume. However, though the surface area and pore volume of V-CS-900 are much larger than V-CS-800, the HMF conversion and DFF yield of V-CS-900 are lower than those of V-CS-800. It is because the aggregation of $\mathrm{VO}_{2}$ in V-CS-900 which is confirmed by the XRD results affects the contact efficiency between $\mathrm{HMF}$ and $\mathrm{VO}_{2}$. In addition, due to the lack of oxygen in the calcination process, the surface area and pore volume of V-CS-900-Ar are much smaller than the catalysts calcined in air, so its activity is much lower than the other catalysts. Therefore, V-CS-800 was the most efficient one for the catalytic oxidation of HMF to DFF. 


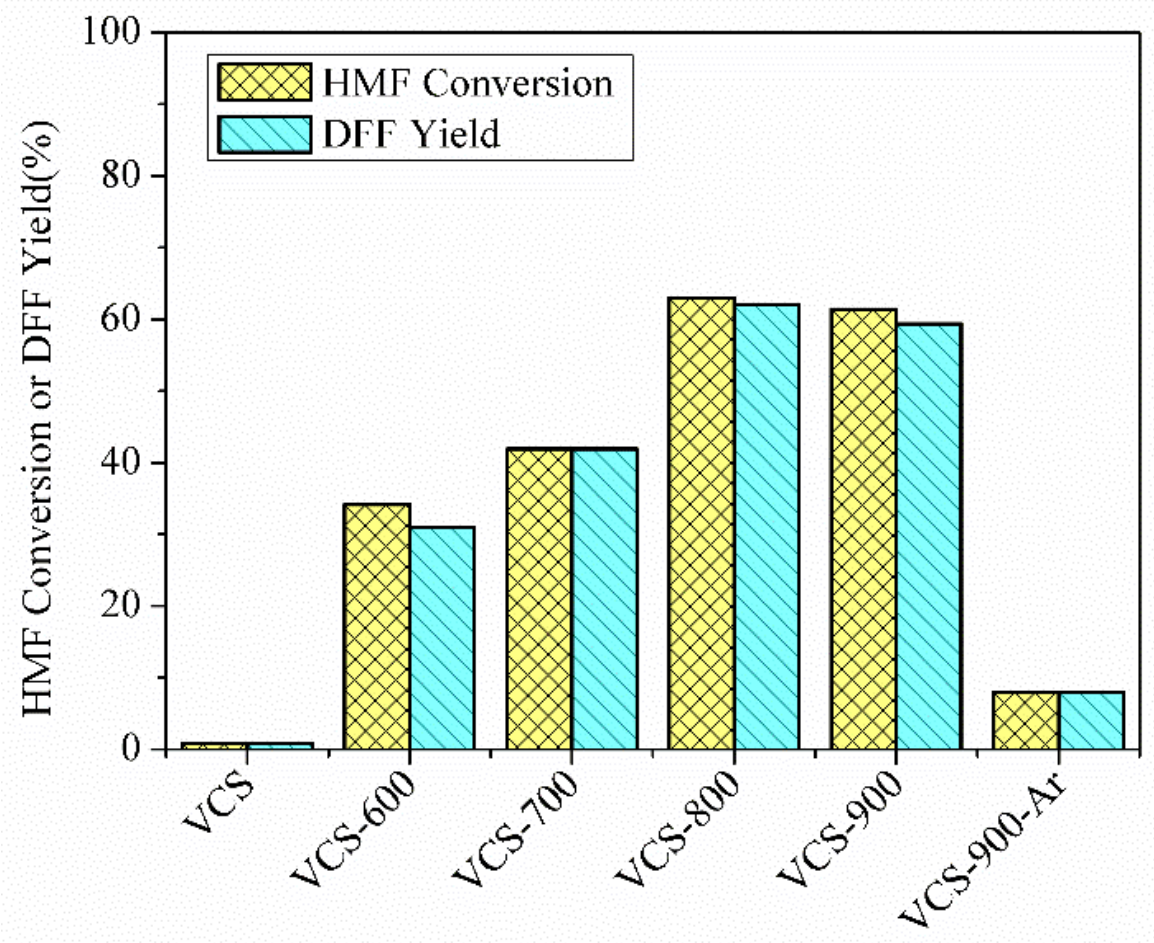

Fig. 5.6 Comparison of HMF oxidation over V-CS thermal treated at different temperatures. Reaction conditions: $\mathrm{DMSO}(5 \mathrm{~mL}), \mathrm{HMF}(0.05 \mathrm{~mol})$, catalyst (V $1.2 \mathrm{mg}), 120^{\circ} \mathrm{C}, \mathrm{O}_{2}(20 \mathrm{~mL} / \mathrm{min}), 4 \mathrm{~h}$.

\subsubsection{Effect of solvents on the catalytic oxidation of HMF to DFF}

To optimize the product selectivity, the oxidation of HMF was conducted in various solvents. It can be seen in Table 5.3 that the HMF conversion and DFF yield were greatly affected by the reaction solvents. With strong polarity and high boiling point, DMSO and DMF were found to be effective for the HMF conversion. This can be attributed to the high oxygen solubility in the polar solvents compared to that in nonpolar solvents. However, DFF yield of $62.95 \%$ obtained from DMSO was much higher than that of $30.34 \%$ observed in DMF under the same reaction conditions. High HMF conversions were also achieved in aromatic solvents such as toluene and dimethylbenzene, but there was a large range between the selectivities of DFF. HMF conversions were lower in other solvents such as Isoamyl alcohol and N-Methyl-2-pyrrolidone, which might be the result of strong interactions between the solvent and the catalytic active sites 
since the heteroatoms $\mathrm{N}, \mathrm{O}$ in the solvent molecules can work as the electron donor. Notably, toluene (nonpolar solvent) afforded HMF conversion of 99\% which is higher than that for DMSO and DMF under the investigated conditions. This result could be possibly attributed to the following reason. The active sites $\left(\mathrm{VO}_{2}\right)$ were located in the organic carbon layer, which make it easier for the aromatic solvents with low polarity to bring the HMF to the activity sites, leading to high reaction efficiency. Therefore, according to the above results, it is difficult to get a direct correlation between the polarity of the solvent and the performance of the catalysts, which is consistent with the conclusion reported by Riisager et al[398] and Chen et al[393]. However, considering the HMF conversion and DFF yield together, DMSO was the optimal solvent for the aerobic oxidation of HMF to DFF.

Table 5.3 Effects of solvents on the aerobic oxidation of HMF to DFF

\begin{tabular}{|c|c|c|c|c|}
\hline Entr & solvent & $\begin{array}{l}\text { HMF } \\
\text { Conversion } \\
(\%)\end{array}$ & $\begin{array}{c}\text { DFF } \\
\text { Yield }(\%)\end{array}$ & $\begin{array}{c}\text { DFF } \\
\text { Selectivity }(\%)\end{array}$ \\
\hline 1 & DMSO & 63.00 & 62.95 & 99.91 \\
\hline 2 & $\mathrm{DMF}$ & 88.19 & 30.34 & 34.40 \\
\hline 3 & Toluene & 99.00 & 43.89 & 44.33 \\
\hline 4 & Isoamyl alcohol & 10.12 & 5.11 & 50.51 \\
\hline 5 & $\begin{array}{l}\text { N-Methyl-2- } \\
\text { pyrrolidone }\end{array}$ & 16.27 & 3.09 & 19.02 \\
\hline 6 & Dimethylbenzene & 55.83 & 1.51 & 2.69 \\
\hline
\end{tabular}

Reaction conditions: Solvent $(5 \mathrm{~mL}), \operatorname{HMF}(0.05 \mathrm{~mol}), \mathrm{V}-\mathrm{CS}-800(35 \mathrm{mg}), 120^{\circ} \mathrm{C}$, $\mathrm{O}_{2}(20 \mathrm{~mL} / \mathrm{min}), 4 \mathrm{~h}$.

\subsubsection{Effect of temperature and time on the catalytic oxidation of HMF to DFF}

Fig.5.7 shows the influence of the reaction temperature and time on the HMF oxidation to DFF over V-CS-800 in DMSO. The reactions were conducted at 
$100^{\circ} \mathrm{C}, 120^{\circ} \mathrm{C}, 140^{\circ} \mathrm{C}$ and $160^{\circ} \mathrm{C}$ spanning a 30 -hour time period. The HMF conversion and DFF yield were obtained in $12.85 \%$ and $11.39 \%$ respectively when the reaction was carried out at the reaction temperature of $100^{\circ} \mathrm{C}$ after $2 \mathrm{~h}$. Further increasing the reaction temperature to $120^{\circ} \mathrm{C}, \mathrm{HMF}$ conversion increase to $42.78 \%$ at the same reaction time. Moreover, the DFF yield sharply increased from $11.39 \%$ at $100^{\circ} \mathrm{C}$ to $74.82 \%$ at $160^{\circ} \mathrm{C}$ in $2 \mathrm{~h}$. As expected, the increase of the reaction temperature dramatically promoted the aerobic oxidation of HMF to DFF, because the increase of the reaction temperature accelerated the movement and contact of the reactant molecules.

In addition to the effects of the reaction temperature on HMF conversion to DFF, the influence of reaction time on the aerobic oxidation of HMF was also investigated under different reaction temperature. It can be seen in Fig.5.7 that the time-changing trend of HMF conversion and DFF yield is greatly affected by the reaction temperature. When the reaction was carried out at $100^{\circ} \mathrm{C}$, the $\mathrm{HMF}$ conversion and DFF yield increase over time - specifically, HMF conversion and DFF yield were $12.5 \%$ and $11.39 \%$ respectively after $2 \mathrm{~h}$ while these values increased to $81.74 \%$ and $79.85 \%$ after $30 \mathrm{~h}$. Similar phenomenon occurred when the reaction temperature rise to $120^{\circ} \mathrm{C}$. HMF conversion and DFF yield reached $86.34 \%$ and $85.79 \%$ respectively in $8 \mathrm{~h}$, then the growth trend slowed dramatically. However, when the reaction temperature was higher than $140^{\circ} \mathrm{C}$, there would be a turning point in the curve of DFF yield versus time though the HMF conversion kept growing as the time went by. A DFF yield of $76.80 \%$ was achieved in $8 \mathrm{~h}$ at $140^{\circ} \mathrm{C}$, then the DFF yield started to decrease. Further raising the reaction temperature to $160^{\circ} \mathrm{C}$, the inflection point for the growth of DFF yield appeared earlier and subsequently declined by up to 17 percent after $30 \mathrm{~h}$. The above results about the influences of reaction temperature and time therefore demonstrated that the optimal temperature for selective oxidation of HMF to DFF 
is $120^{\circ} \mathrm{C}$, as higher reaction temperature could cause the over oxidation of HMF to by-products such as FFCA and FDA after a long time reaction[399].
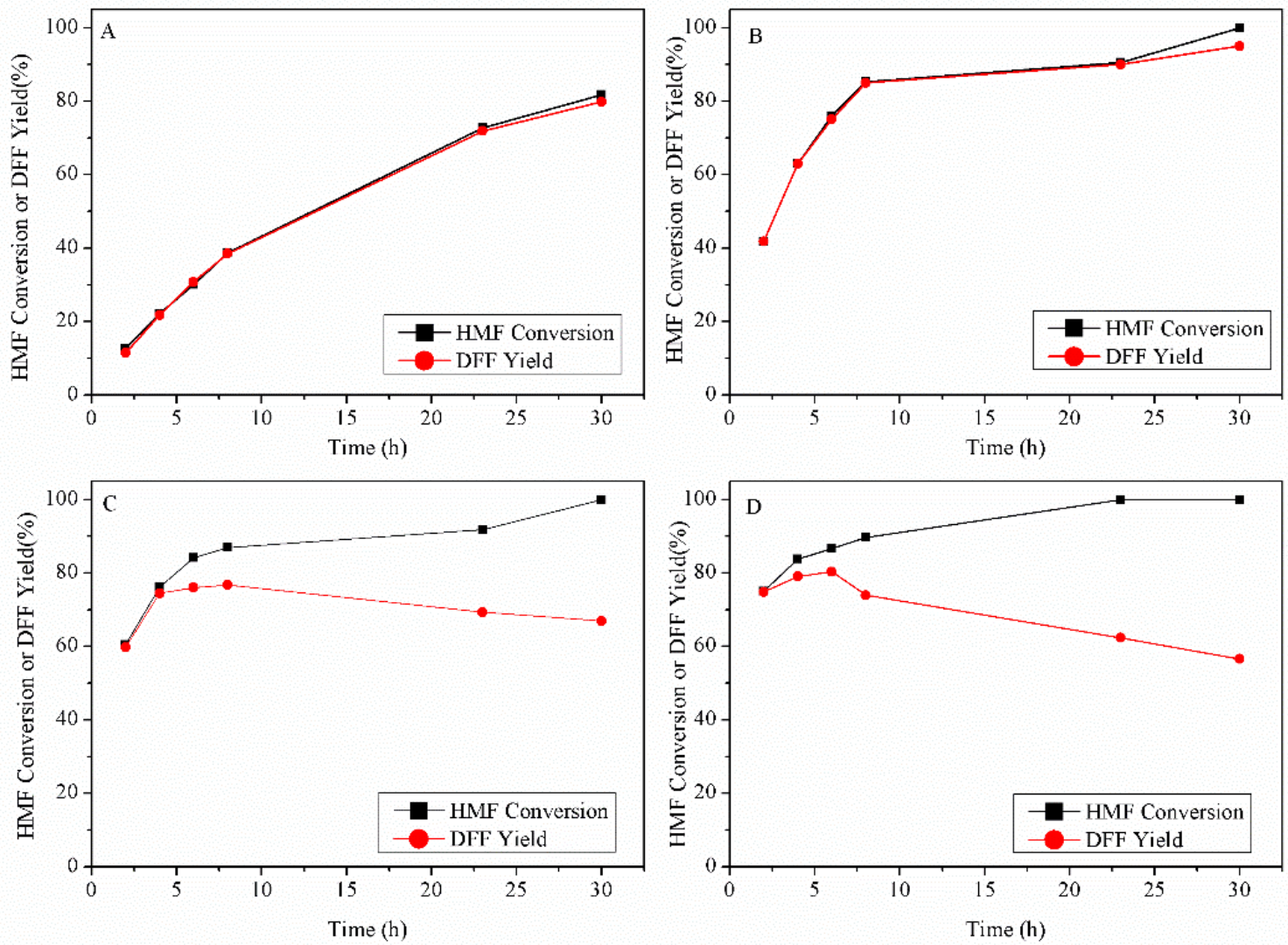

Fig.5.7 Effect of temperature and time on the catalytic oxidation of HMF to DFF. Reaction conditions: DMSO(5mL), HMF(0.05mol), V-CS-800(35mg), $\mathrm{O}_{2}(20 \mathrm{~mL} / \mathrm{min})$, reaction temperature (A) $100{ }^{\circ} \mathrm{C}$, (B) $120{ }^{\circ} \mathrm{C}$, (C) $140{ }^{\circ} \mathrm{C}$,(D) $160^{\circ} \mathrm{C}$.

\subsubsection{Effect of catalyst to substrate ratio on the catalytic oxidation of HMF to}

\section{DFF}

The dosage of catalyst was also a crucial factor for optimization of the reaction. Fig.5.8 shows the relations among HMF conversion, DFF yield and ratio of metal to substrate. It can be seen that the HMF conversion and DFF yield increased significantly with increasing the catalyst concentrate. Under the optimal reaction conditions, the HMF conversion and DFF yield reached 100\% and $99 \%$ respectively with the increase of ratio of vanadium to HMF as only 2 wt.\%, indicating the high catalytic efficiency of the catalyst. 


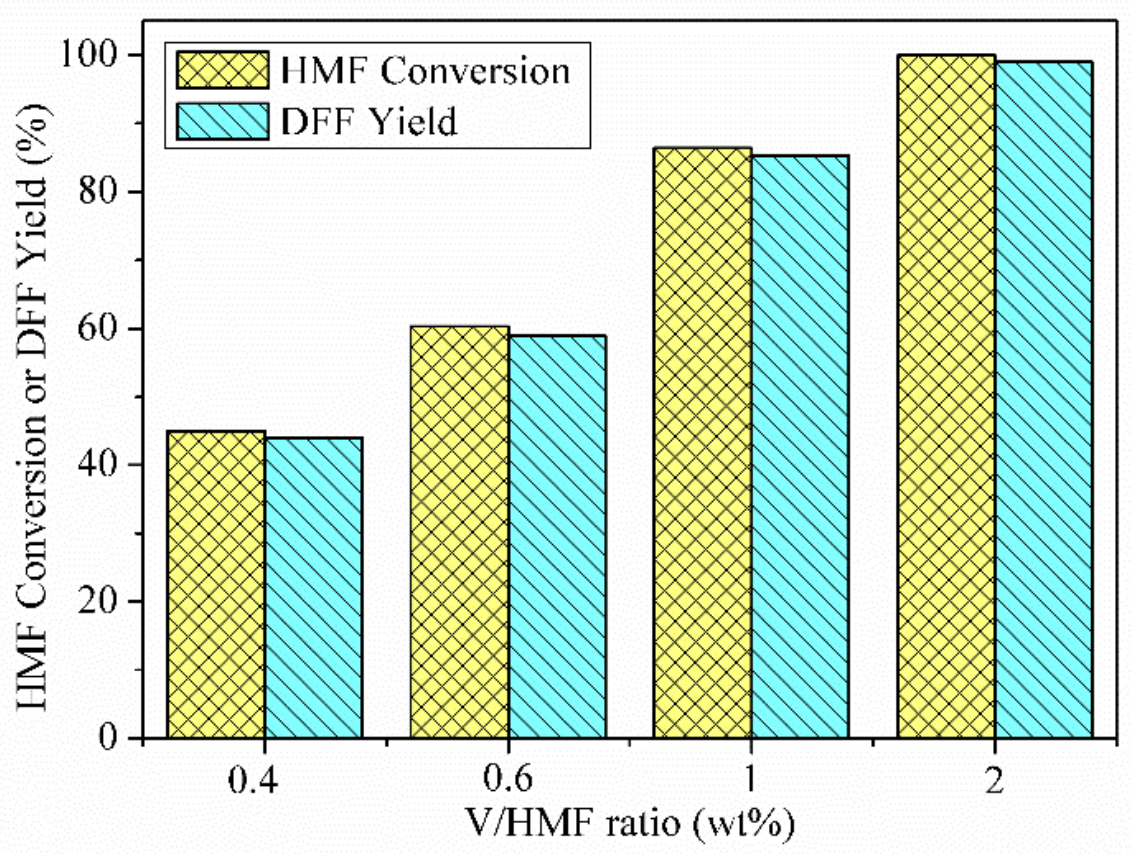

Fig.5.8 Effect of catalyst to substrate ratio on the catalytic oxidation of HMF to DFF. Reaction conditions: $\mathrm{DMSO}(5 \mathrm{~mL}), \mathrm{HMF}(0.05 \mathrm{~mol}), 120^{\circ} \mathrm{C}, \mathrm{O}_{2}(20 \mathrm{~mL} / \mathrm{min}), 9 \mathrm{~h}$.

\subsection{Catalyst recycling experiments}

The reusability of the V-CS- 800 catalyst was investigated by conducting five consecutive runs in DMSO. Before each reuse, the catalyst was washed thoroughly with the corresponding solvent and ethanol and dried overnight at $80^{\circ} \mathrm{C}$. It can be seen from the results showed in Fig. 5.9 that the yield of DFF almost remained unchanged even after being used 5 times. Recently, PVP-1/VO (Vanadyl-pyridine complexes immobilized on a polymeric mesoporous support) was developed by Navarro et al. for HMF oxidation[393]. The performance of the catalyst decreased significantly due to the vanadium leaching. Vanadium phosphate oxide, synthesized by Dumeignil et al. , also showed a decrease in catalyst activity for oxidation of HMF to DFF during the recycling test owing to the change in catalyst structure which reduced to number of accessible reactive vanadium centers[400]. Moreover, Riisager et al. reported the loss of activity of the zeolite-supported vanadium catalyst in the stability test as a result of the vanadium leaching and site blocking phenomena[398]. However, TGA results 
showed that the vanadium amount of V-CS-800 remained about the same after the reusability test, suggesting the strong interaction between the vanadium species and the carbon support.

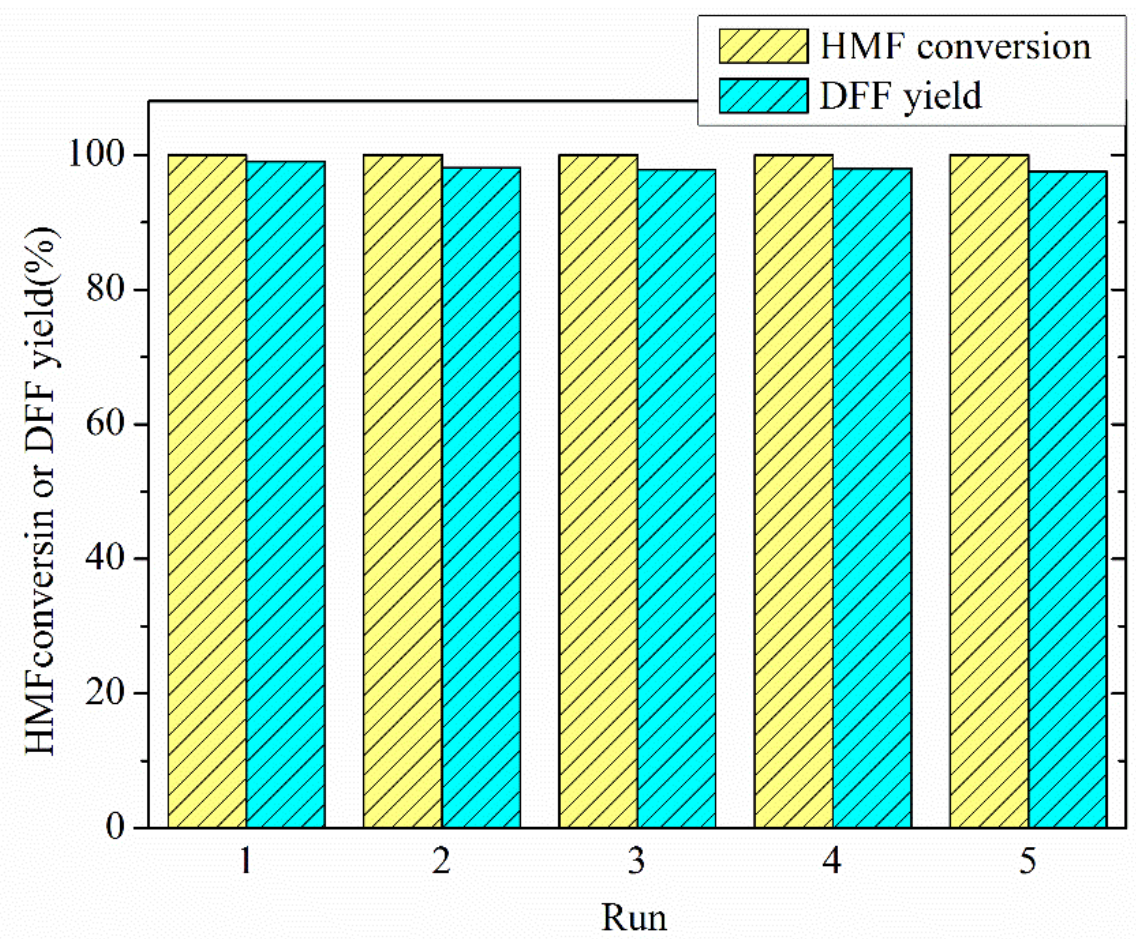

Fig. 5.9 Catalyst V-CS-800 recycling. Reaction conditions: Solvent (5mL), HMF (0.05mmol), V-CS-800(65mg), $120^{\circ} \mathrm{C}, \mathrm{O}_{2}(20 \mathrm{~mL} / \mathrm{min}), 9 \mathrm{~h}$.

\subsection{Summary}

In summary, vanadium-embedded mesoporous carbon microspheres were synthesized by a facile hydrothermal method. The nonporous V-CS catalyst was activated by thermal treatment to introduce micro- and mesoporous structure, leading to the increase in specific surface from 60 to $934 \mathrm{~m}^{2} / \mathrm{g}$. Under appropriate conditions, the vanadium catalyst (V-CS-800) showed homogeneously dispersed $\mathrm{VO}_{2}$ nanoparticles with in the mesoporous carbon matrixes. The as-prepared vanadium catalyst was highly selective for catalytic aerobic oxidation of HMF to DFF using molecular oxygen as oxidant. The high activity was attributed to the mesoporous structure of the catalyst which allowed the free movements of reactants and products and enhanced the contact chances between reactants and active sites. Different reaction conditions were investigated to increase the yield 
of DFF. The effects of solvents, reaction time, temperature and ratio of catalyst to substrate were studied. The choice of solvent also had a very important effect on the reusability and stability of the catalyst though the reason behind is remaining not clear. Under optimized reaction conditions, HMF conversion of $100 \%$ and DFF yield of more than $99 \%$ were achieved when the $2 \mathrm{wt} . \%$ (V/HMF) V-CS-800 was applied as the catalyst in DMSO at $120^{\circ} \mathrm{C}$ and oxygen bubbling at a flow rate of $20 \mathrm{~mL} / \mathrm{min}$ after $9 \mathrm{~h}$. In addition, there was little decline in the catalytic performance in the recycling experiments, indicating the excellent stability and reusability of V-CS-800. In short, this novel step-economic synthetic strategy can facilely produce efficient catalyst for biomass-based conversion. 


\section{Chapter 6 Molybdenum Trioxide Supported on Carbon Sphere for One-}

\section{Step Approach to 2,5-Diformylfuran from Fructose}

In this chapter, a series of molybdenum oxide species supported on carbon spheres (Mo-CS) were developed and used catalysts in the direct synthesis of 2,5diformylfuran(DFF) from fructose with molecular oxygen as the oxidant and dimethylsulfoxide as solvent. Different aftertreatment methods were applied to the solid Mo-CS precursor to strengthen the sphere structure, generate micro-and mesopores within the catalyst and change the oxidation states of encapsulated molybdenum in order to enhance the performance in the one-step conversion of fructose to DFF. The structure and properties of the resultant catalysts was characterized by X-ray diffraction(XRD), element analysis, Raman spectroscopy, Field emission scanning electron microscopy (FESEM), Fourier transform infrared (FTIR) spectra, Brunauer-Emmett-Teller (BET) Surface Area Analysis, and ammonia temperature-programmed desorption (NH3-TPD). The assynthesized catalysts showed high activity and selectivity towards direct transformation from fructose to DFF. A $100 \%$ conversion of fructose can be obtained within $2 \mathrm{~h}$ at $160^{\circ} \mathrm{C}$ in DMSO and the corresponding DFF yield can reach as high as $77 \%$, which is a very satisfying result. Moreover, the heterogenized catalysts could be recycled and reused for at least 5 runs without significant loss of activity.

\subsection{Catalyst characterization}

Uniform spherical carbon sphere could be prepared by hydrothermal method in the temperature range of $160-200^{\circ} \mathrm{C}$ with carbohydrates as the carbon precursor, while the preparation of this type of metallic-carbon hybrids with homogeneously distributed metal species is still quite challenging[389]. Fig.6.1 shows the morphology of as synthesized carbon sphere supported molybdenum oxide catalysts prepared at $180 \mathrm{oC}$ for $12 \mathrm{~h}$ under hydrothermal conditions and followed by different after-treatments. As can been seen, the spherical catalysts 
have a uniform size distribution of around $1 \mu \mathrm{m}$ (Fig. 6.1a). after carbonization in nitrogen at $750^{\circ} \mathrm{C}$ or calcination in air at $275^{\circ} \mathrm{C}$ for $3 \mathrm{~h}$, the spherical morphology remains unchanged, but the size decrease to less than $1 \mu \mathrm{m}$, which is probably due to the gasification of carbon at high temperature especially when oxygen exists in the environment[394]. It is worth noting that the surface of Mo$\mathrm{CS}$ was smooth and it remains smooth even after annealing in nitrogen at $750^{\circ} \mathrm{C}$ for 3h. However, Mo-CS-275, which was obtained from calcination of Mo-CS in air, has obviously rough and grainy surface. This is because of the remove of carbon component by the reaction between the carbon atoms or fragments and oxygen in air atmosphere leads to the explosion of molybdenum oxide species.
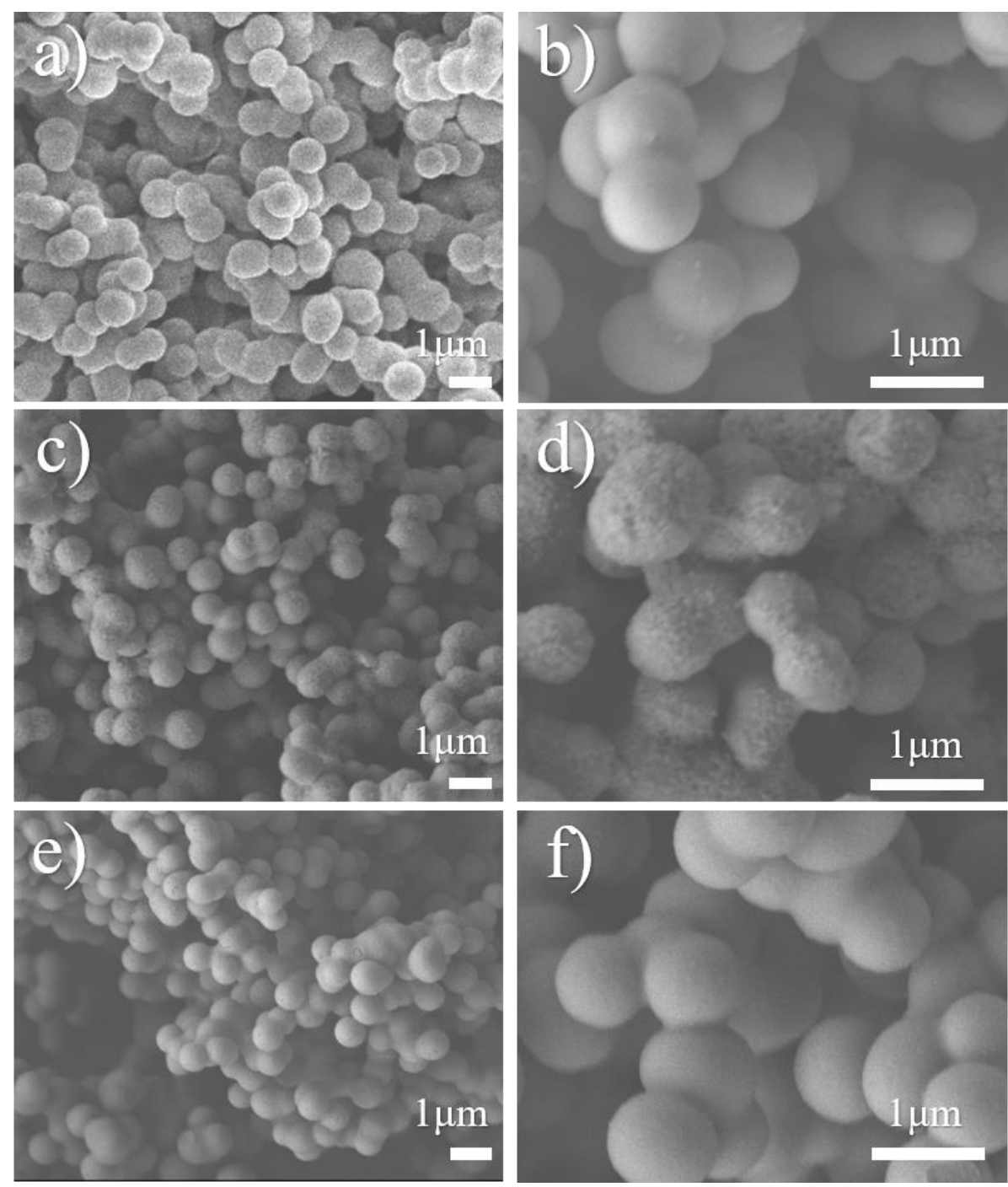

Fig.6.1 FESEM images of Mo-CS (a,b), Mo-CS-275 (c,d), Mo-CS-N2 (e,f). 
X-ray powder diffraction was carried out to study the crystallographic structure of the as-synthesized catalysts. According to the study by Alexander Briceno, molybdenum was reduced by glucose from the oxidation state ${ }^{+} 6$ in its precursor phosphomolybdic acid to the ${ }^{+} 4$ in $\mathrm{MoO}_{2}$ [401]. Due to the homogeneous distribution of molybdenum oxide species in carbon spheres, no obvious peaks of $\mathrm{MoO}_{2}$ are observed in Fig. 6. 2 for the sample Mo-CS. For the same reason, similar behaviors are also found for the sample Mo-CS-N2, in which the molybdenum oxide species may be further reduced and carbonized to hexagonal molybdenum carbide after calcination at $750^{\circ} \mathrm{C}$ in nitrogen[402]. However, after calcination in air, the crystallinity of molybdenum oxide species was significantly improved. A considerable amount of carbon atoms or fragments were removed and the molybdenum oxide species were oxidized to orthorhombic molybdenum trioxide with major diffraction peaks as shown in Fig. 6. 2[403]. There are no graphitic carbon peaks observed, since the carbonaceous phase in these carbon spheres are amorphous which is confirmed by the broad peak between $20-30^{\circ}$. This peak attributed to 002 interlayer reflection in the carbon packing and might reflect the average separation distance between polycyclic aromatic carbon segments[404].

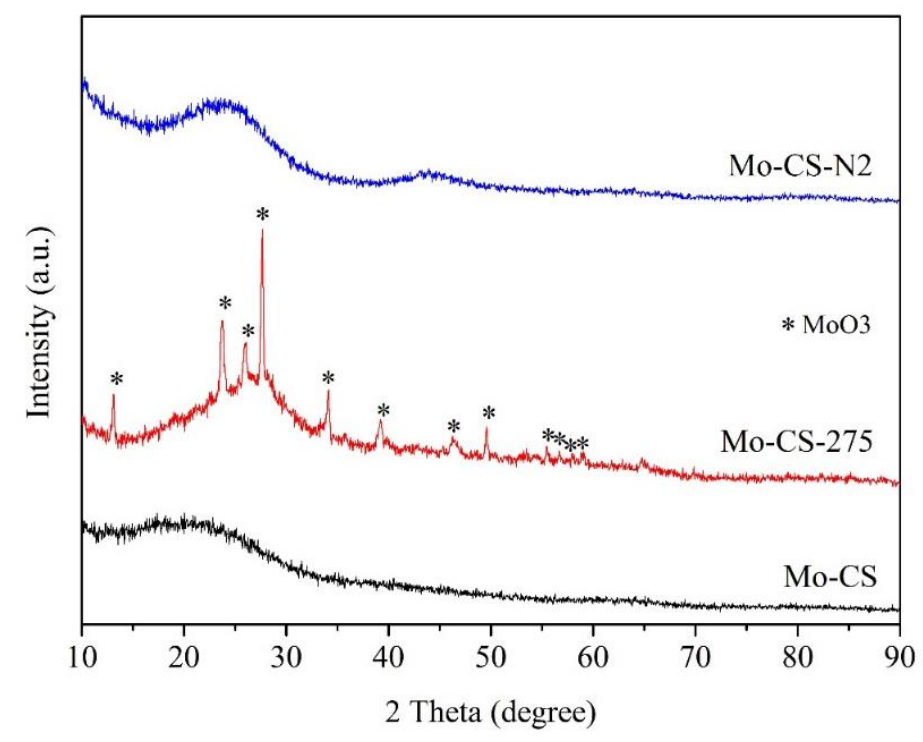

Fig. 6. 2 XRD analysis of Mo-CS, Mo-CS-275 and Mo-CS-N2. 


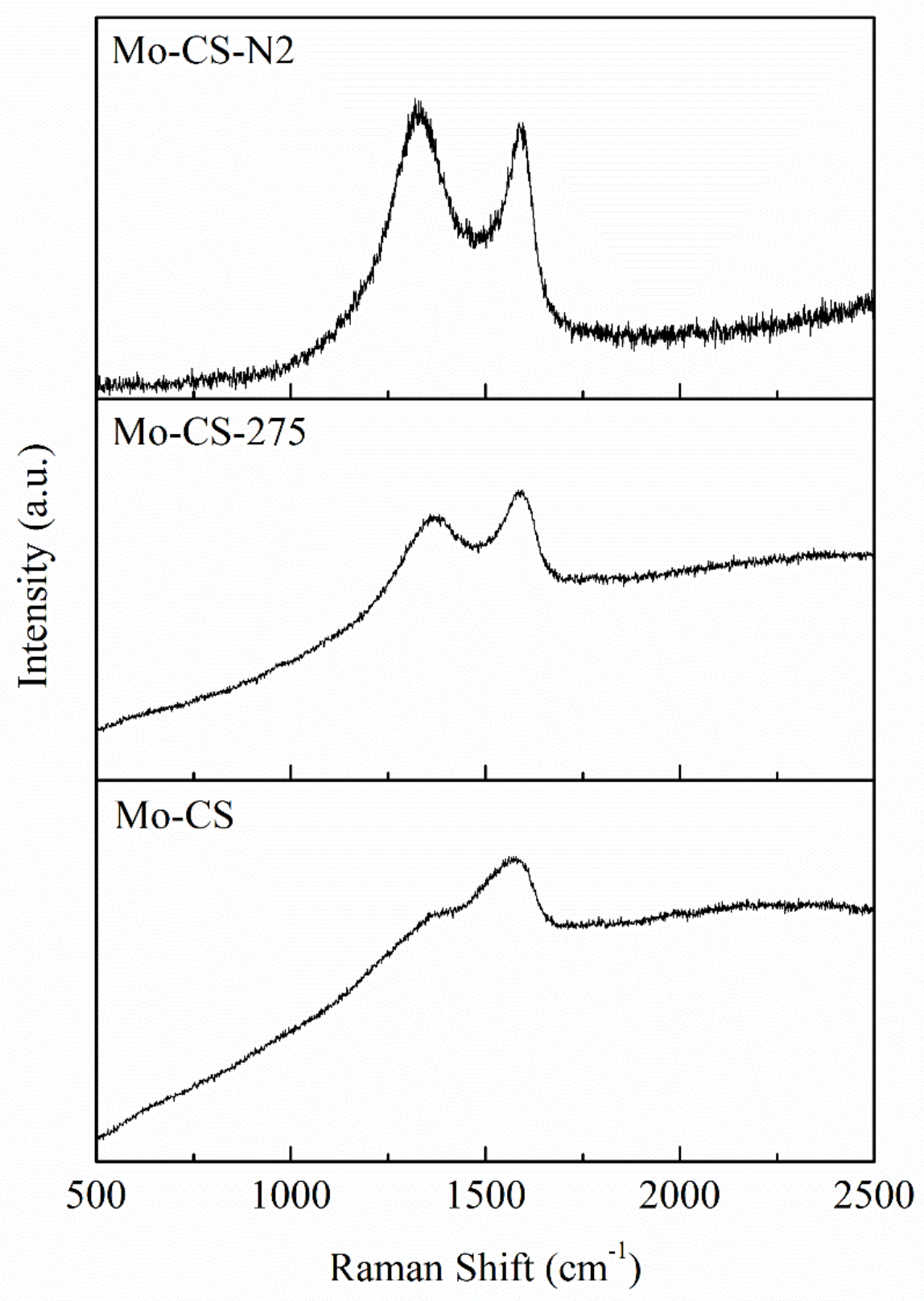

Fig. 6.3 Raman spectrum of Mo-CS, Mo-CS-275, Mo-CS-N2.

The Raman spectrums of carbon sphere supported molybdenum oxide samples are shown in Fig. 6.3. Two typical peaks at $\sim 1300 \mathrm{~cm}-1$ and $\sim 1600 \mathrm{~cm}-$ 1 are observed for all the as-synthesized samples. The peak at $\sim 1300 \mathrm{~cm}-1$ also known as D-band is attributed to the vibration of carbon atoms with dangling bonds, while the G-band ( 1600 cm-1) corresponds to the in-plane E2g zonecenter mode[392]. The intensity ratios of the D- and G- bands are larger than 0.5, 
reflecting the low degree of graphitization of the carbon sphere and the presence of high contents of lattice edges and defects in the disordered carbon[405, 406], which is consistent with the XRD results. Moreover, this ratio ID/IG increased after calcination, indicating that high temperature carbonization enhanced the degree of disorder.

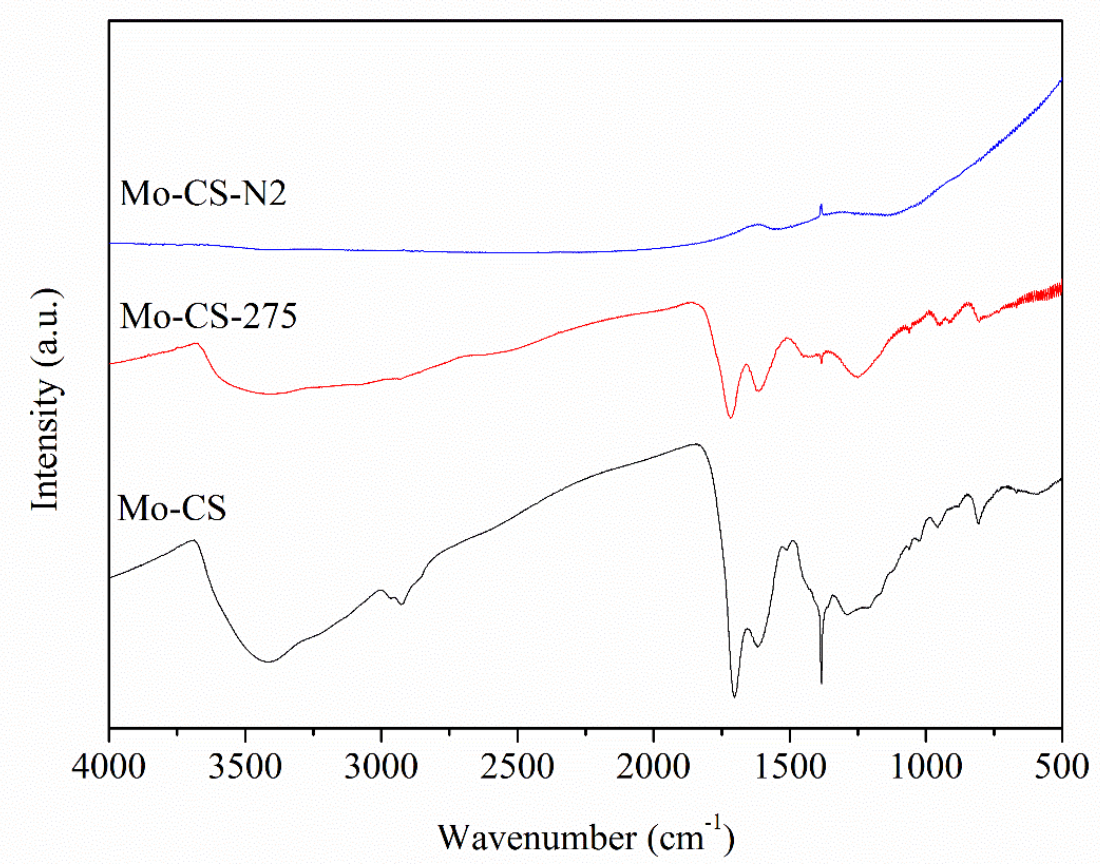

Fig. 6.4 FTIR spectra of Mo-CS, Mo-CS-275 and Mo-CS-N2.

FTIR spectroscopy was utilized to study the assignment of surface functional groups of the as-synthesized catalysts (Fig. 6.4). Generally, all the peaks become less obvious after calcination at $750^{\circ} \mathrm{C}$ in nitrogen indicating the shedding of the functional groups due to high temperature. The broad band centered around $3400 \mathrm{~cm}-1$ is attributed to the O-H groups. The peaks at 2920 and $1380 \mathrm{~cm}-1$ correspond to the stretching and bending modes of $\mathrm{C}-\mathrm{H}$ groups. Compared with Mo-CS, the intensity of these peaks of Mo-CS-275 and Mo-CS-N2 decreased after the calcination. In contrast, the intensity of the peak corresponding to C-O groups (1000-1300cm-1) increased after calcination in air. The peaks at $1702 \mathrm{~cm}-$ 1 and $1619 \mathrm{~cm}-1$ can be assigned to the $\mathrm{C}=\mathrm{O}$ vibration of ketones, esters, 
aldehydes or carboxylic acid and $\mathrm{C}=\mathrm{C}$ bond of alkenes or aromatic rings respectively[407]. Element analysis were carried out to study the chemical components (i.e. carbon, hydrogen, oxygen) of the catalysts and the results were consistent with the FTIR analysis. It can be seen that there are great differences in chemical composition between different samples in Table 6.1. Mo-CS-N2 has a much higher percentage of carbon and less level of hydrogen and oxygen than the other two types of catalysts because of the loss of hydrocarbon group and carbon-oxygen groups under high temperature during the calcination in nitrogen. Meanwhile, when Mo-CS was annealing in air, the oxidation reaction between carbon atoms and oxygen lead to a higher oxygen-to-carbon ratio in Mo-CS-275 than Mo-CS and Mo-CS-N2. Moreover, the calcination also caused the increase of molybdenum content because of the consumption of carbon atoms in the process.

Table 6.1 Elemental composition and surface areas of Mo-CS, Mo-CS-275 and Mo-CS-N2.

\begin{tabular}{|c|c|c|c|c|c|}
\hline Catal & Carbon $^{\mathrm{a}}$ & Hydroge & Oxygen & $\mathrm{Mo}^{\mathrm{b}} /$ & $\mathrm{BET} / \mathrm{m}$ \\
\hline yst & $1 \%$ & $\mathrm{n}^{\mathrm{a}} \%$ & $\mathrm{a} \%$ & $\%$ & $2 / g$ \\
\hline $\begin{array}{l}\text { Mo- } \\
\mathrm{CS}\end{array}$ & 63.36 & 4.95 & 29.02 & 2.67 & $<1$ \\
\hline $\begin{array}{r}\text { Mo- } \\
\text { CS-275 }\end{array}$ & 44.74 & 2.21 & 46.27 & 6.78 & 28 \\
\hline $\begin{array}{r}\text { Mo- } \\
\text { CS-N2 }\end{array}$ & 81.11 & 1.50 & 13.11 & 4.28 & 427 \\
\hline
\end{tabular}

${ }^{\text {a }}$ Determined by elemental analysis

${ }^{\mathrm{b}}$ Determined by TGA 


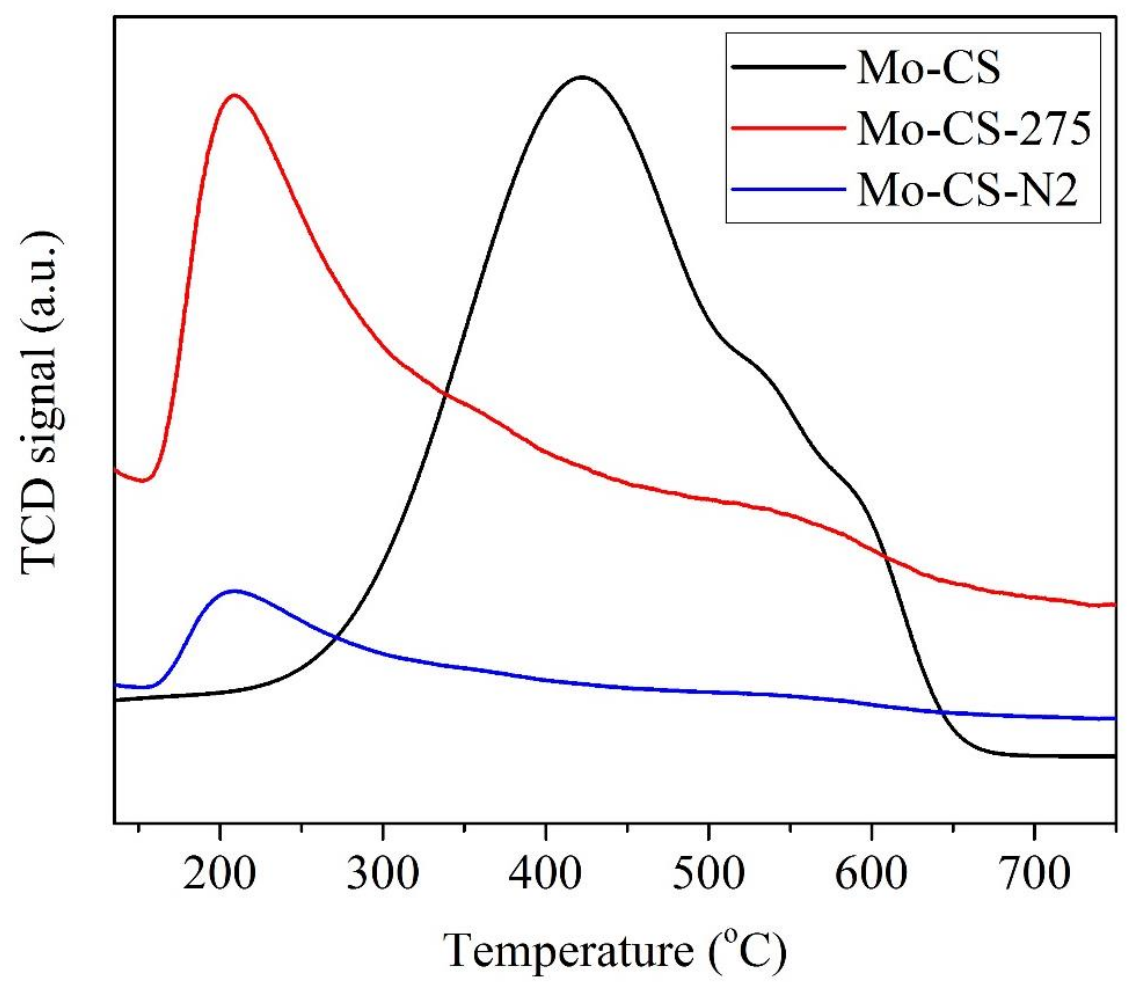

Fig. 6.5 NH3-TPD curves of Mo-CS, Mo-CS-275 and Mo-CS-N2

Since the dehydration of fructose to HMF is an acid catalyzed reaction, the acidity of the catalysts should be one of the key factors which influence the catalytic activity. Thus, NH3-TPD examination was carried out to study the acidity of the catalysts(Fig.6.5). It can be seen that there is a big peak ranging from $250^{\circ} \mathrm{C}$ to $650^{\circ} \mathrm{C}$ for Mo-CS, indicating the rich Bronsted and Lewis acid sites on the surface, which are attributed to the various surface functional groups such as hydroxyl, carboxyl groups and the molybdenum oxide species[408]. This peak disappeared for the samples after calcination, while a new peak around $220^{\circ} \mathrm{C}$ was observed for Mo-CS-275 and Mo-CS-N2. It should be noted that the peak intensity of Mo-CS-275 is much higher than that of Mo-CS-N2. Generally, the high-temperature peak is related to medium acid sites and the lowtemperature is associated with weak temperature. Therefore, the heat treatment of the Mo-CS leads to the loss of medium acid sites and the formation of weak 
acid sites due to the change of the surface groups and the oxidation state of molybdenum, which is line with the analysis of FTIR results.

\subsection{Catalytic performance of catalysts}

\subsubsection{Dehydration of fructose of $\mathrm{HMF}$}

A series of experiments were conducted to initially investigate the catalytic performance of the carbon sphere supported molybdenum oxide catalysts towards fructose dehydration. In Fig. 6.6, all the tested catalysts showed good activity and both the fructose conversion and HMF yield increased with reaction time. However, Mo-CS-275 obviously showed better performance than the other two types of catalysts especially at the early stage of the dehydration reaction. It can be seen that the yield of HMF can reach more than $70 \%$ in only $2 \mathrm{~h}$ in the presence of Mo-CS-275, whereas this value was just $52.69 \%$ and $62.03 \%$ by using Mo-CS-N2 and Mo-CS respectively. The same goes for the fructose conversion rate, when the reaction was conducted in the presence of Mo-CS-275, the fructose conversion increased to $97.02 \%$ after $2 \mathrm{~h}$, while it took 4-6h for Mo$\mathrm{CS}$ and Mo-CS-N2 to catalyze the reaction and get the comparable fructose conversion under the same condition. In general, Mo-CS-275 showed the best catalytic activity for the dehydration of fructose to HMF duo to its relatively high acid concentration and large BET surface area comparing with Mo-CS-N2 whose acid amount is too little and Mo-CS who has a very small BET surface area which cannot provide enough contact chance for the active site and reactant. 


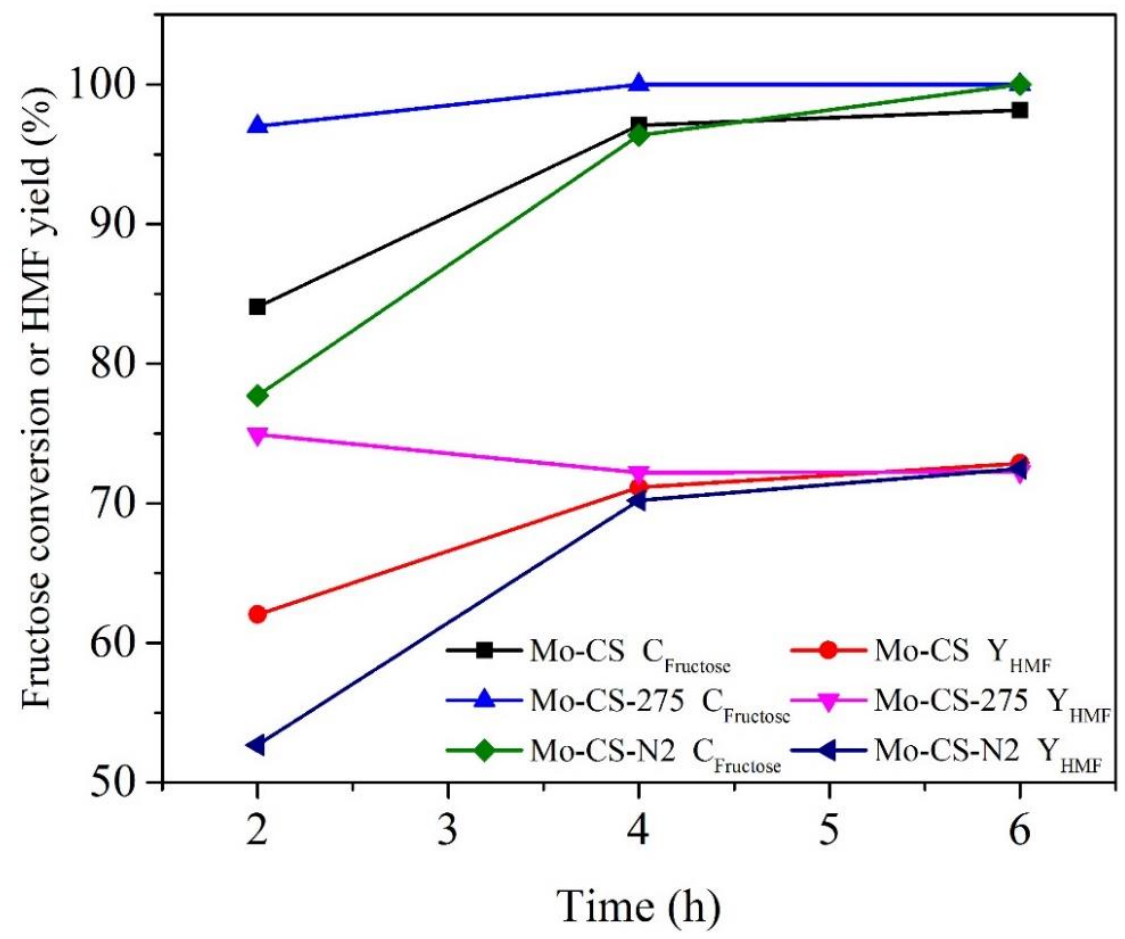

Fig. 6.6 Dehydration of fructose to HMF over Mo-CS, Mo-CS-275 and MoCS-N2. Reaction conditions: fructose (200mg), catalyst (30mg), DMSO (5mL), $110^{\circ} \mathrm{C}$.

\subsubsection{Aerobic oxidation of HMF to DFF}

After the investigation of the catalysis performance for fructose dehydration to $\mathrm{HMF}$, aerobic oxidation of HMF to DFF was further explored in order to select the best catalyst for one-step approach transformation form fructose to DFF. It was found that the catalytic performances of the as-synthesized catalysts varied greatly with different after-treatments for the metallic sites and the carbon support as shown in Fig. 6.7. HMF conversion of $31.96 \%$ and DFF yield of $30.83 \%$ were obtained after $2 \mathrm{~h}$ in DMSO at $140^{\circ} \mathrm{Cwith}$ oxygen bubbling rate as $20 \mathrm{~mL} / \mathrm{min}$, whereas these values were less than $10 \%$ when Mo-CS or Mo-CSN2 was used as the catalyst. Moreover, when the reaction time was further prolonged to $8 \mathrm{~h}$, the results gap between different catalysts became much more evident. The conversion of HMF and yield of DFF increased to $89.36 \%$ and $86.74 \%$ respectively after $8 \mathrm{~h}$ in the presence of Mo-CS-275. In sharp contrast, HMF 
conversion and DFF yield of $45.60 \%$ and $43.38 \%$ respectively were observed from Mo-CS-N2, and these values can be much less for Mo-CS. Above results indicate that the catalytic performance was influence dramatically by the molybdenum oxide species state and BET area. The catalytic effect of Mo-CS was limited by the low surface area, while the low valence state of Mo in MoCS-N2 is related to the low reaction activity towards the aerobic oxidation of HMF to DFF.

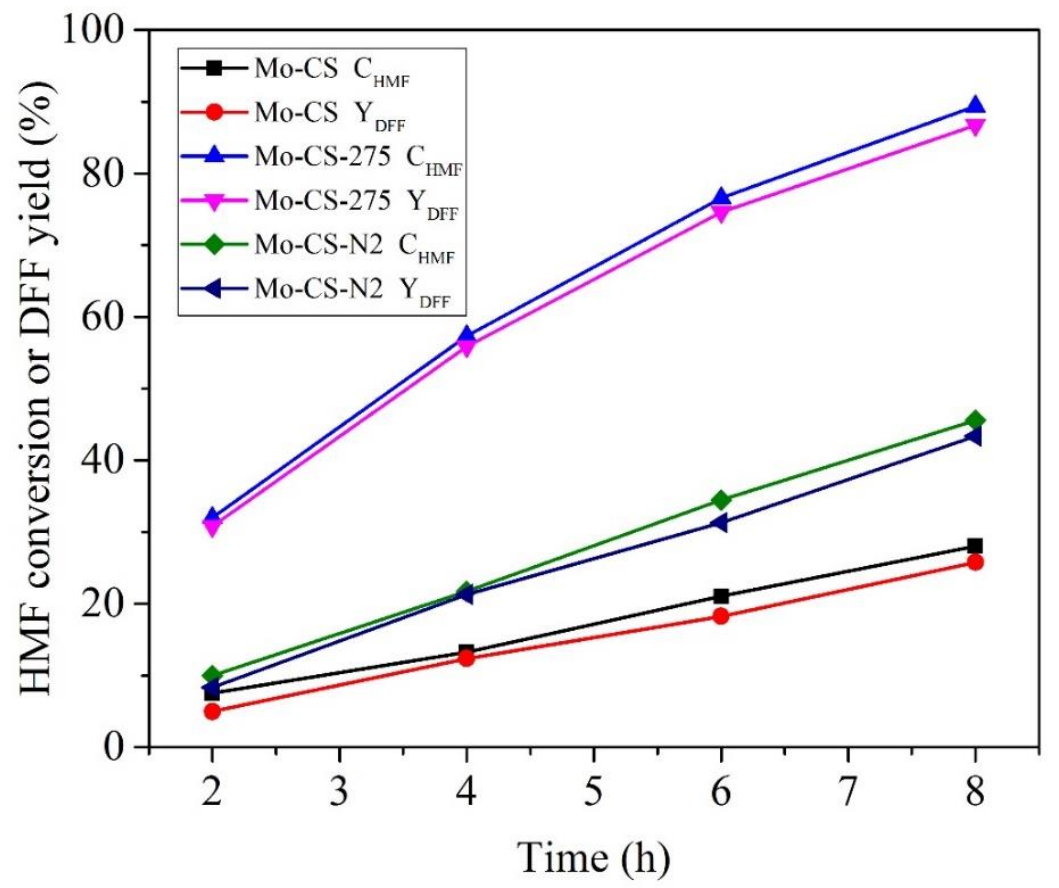

Fig. 6.7 Aerobic oxidation of HMF to DFF over Mo-CS, Mo-CS-275 and Mo-CS-N2. Reaction conditions: HMF (63mg, 0.5mmol), catalyst (30mg), $\mathrm{O}_{2}(20 \mathrm{~mL} / \mathrm{min}), \mathrm{DMSO}(5 \mathrm{~mL}) 140^{\circ} \mathrm{C}$.

\subsubsection{One-pot conversion of fructose to DFF}

Based on two separate studies on the catalytic performance of Mo-CS, MoCS-275 and Mo-CS-N2 in fructose dehydration to HMF and HMF oxidation to DFF, Mo-CS-275 was supposed to be the best choice for direct conversion of fructose to DFF. Thus one-pot synthesis of DFF from fructose was carried out by using the as-synthesized catalysts to further confirm the preliminary prediction. Unlike the one-pot but two-step reaction, in which the dehydration of fructose to 
HMF was performed under inert gas and the aerobic oxidation of HMF to DFF was performed under oxygen, no inert gas was applied as the protection gas and the reaction system was bubbled with oxygen from beginning to end. Meanwhile, in order to eliminate the effect of molybdenum oxide species loading level, calculated amount of catalysts with equivalent molybdenum content were fed into the reaction. It can be seen in Fig. 6.8 that all the tested catalysts showed good catalytic activity as expected with fructose being $100 \%$ converted and HMF and DFF were detected as the main products. The exist of HMF affirmed the intermediate role of HMF during the transformation of fructose to DFF. In comparison, the yield of target product DFF presented by Mo-CS-275 was higher than that by Mo-CS and Mo-CS-N2 under the investigated conditions, suggesting that Mo-CS-275 was the best catalyst for one-step approach towards DFF from fructose because of its relatively high acid density and strong oxidative ability.

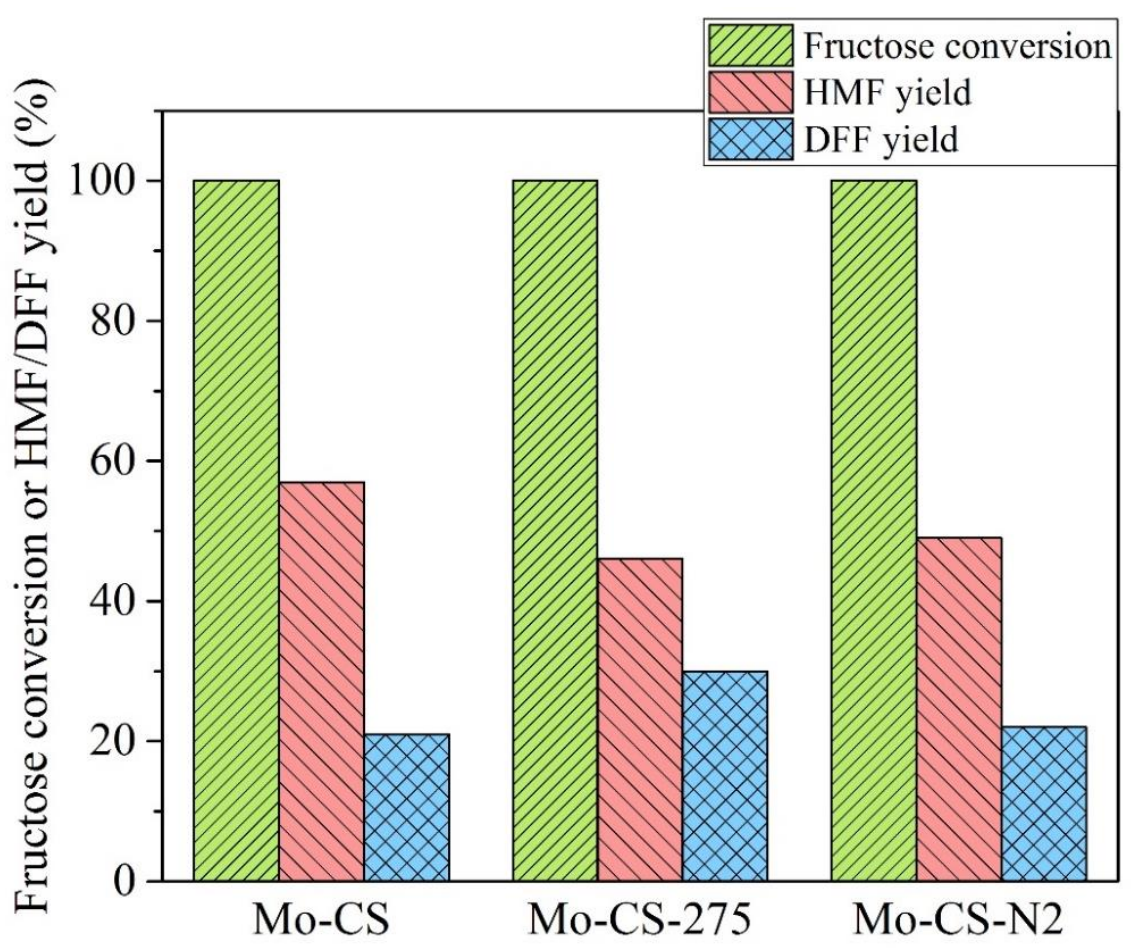

Fig. 6.8 Direct transformation of fructose to DFF catalyzed by Mo-CS, MoCS-275 and Mo-CS-N2. Reaction conditions: fructose (200mg), catalyst (Mo 2mg), DMSO (5mL), $\mathrm{O}_{2}(20 \mathrm{~mL} / \mathrm{min}), 130^{\circ} \mathrm{C}, 8 \mathrm{~h}$ 
Specimens sampled at specified time intervals from the reaction mixture were analyzed to study the time course of the one-pot conversion of fructose to DFF. As shown in Fig. 6.9, fructose dehydrated to HMF quickly that HMF yield could reach nearly $80 \%$ after $1 \mathrm{~h}$ with fructose conversion approaching $100 \%$. As the reaction proceeding, the yield of HMF exhibited a declining tendency and became trace after 30h. Accordingly, the yield of DFF increased from $7.69 \%$ at $2 \mathrm{~h}$ to 77.75 at $30 \mathrm{~h}$. Obviously, the reaction can be divided into two stages, namely the dehydration of fructose to HMF and the accumulation of DFF with destruction of HMF. During the process, the quick dehydration of fructose to HMF considerably reduced the contact time of fructose with oxygen and oxidative active centers which can avoid the large generation of by-products and force the reaction to move into the second stage. As to the second stage, the excellent activity of the catalyst and high selectivity towards DFF in aerobic oxidation of HMF has been proven. They are the reasons why Mo-CS-275 showed superior performance in the one-pot and one-step conversion of fructose to DFF.

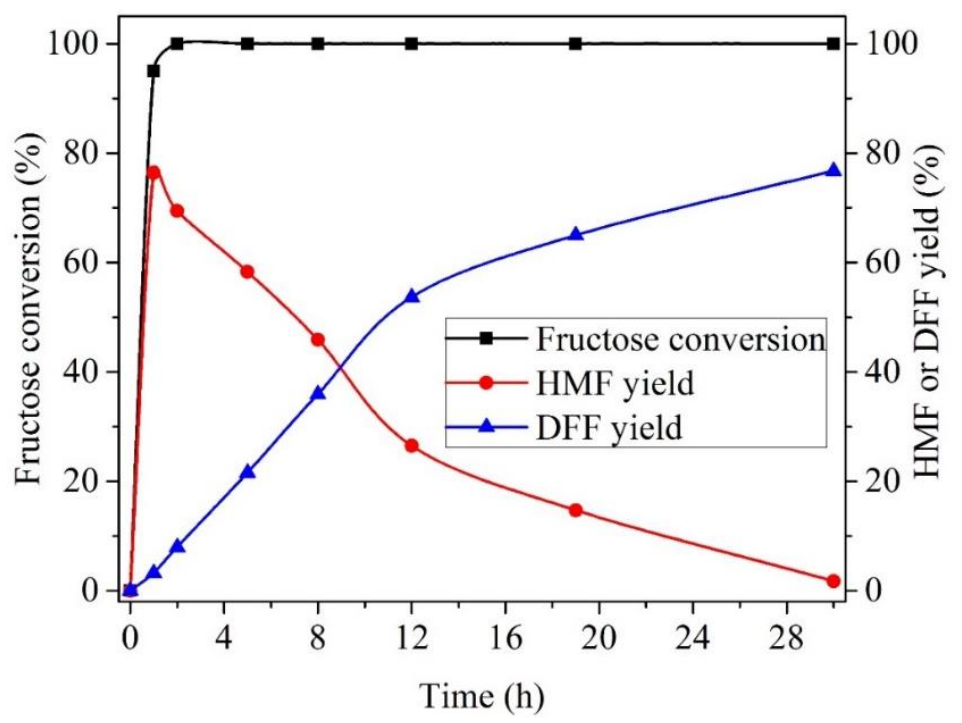

Fig. 6.9 Conversion of fructose to HMF and DFF as a function of time. Reaction conditions: fructose (200mg), CS-Mo-275 (30mg), DMSO (5mL), $\mathrm{O}_{2}$ (20mL/min), $130^{\circ} \mathrm{C}$. 


\subsection{Optimizations of the reaction conditions over Mo-CS-275}

In order to achieve a maximum possible DFF yield, reaction conditions such as temperature, time and ratio of reactant to catalyst were investigated. The effect of weight ratio of Mo to fructose was studied at the ratio of $0.4,1 \%, 2 \%$ and $4 \%$ under similar conditions. Overall, the results showed the catalyst work steadily with a remarkable performance (Fig. 6.9). 57.78\% DFF yield and 20.16\% HMF yield were obtained after $18 \mathrm{~h}$ under $130^{\circ} \mathrm{C}$ in DMSO even though the ratio of Mo to fructose was only $1 \%$.

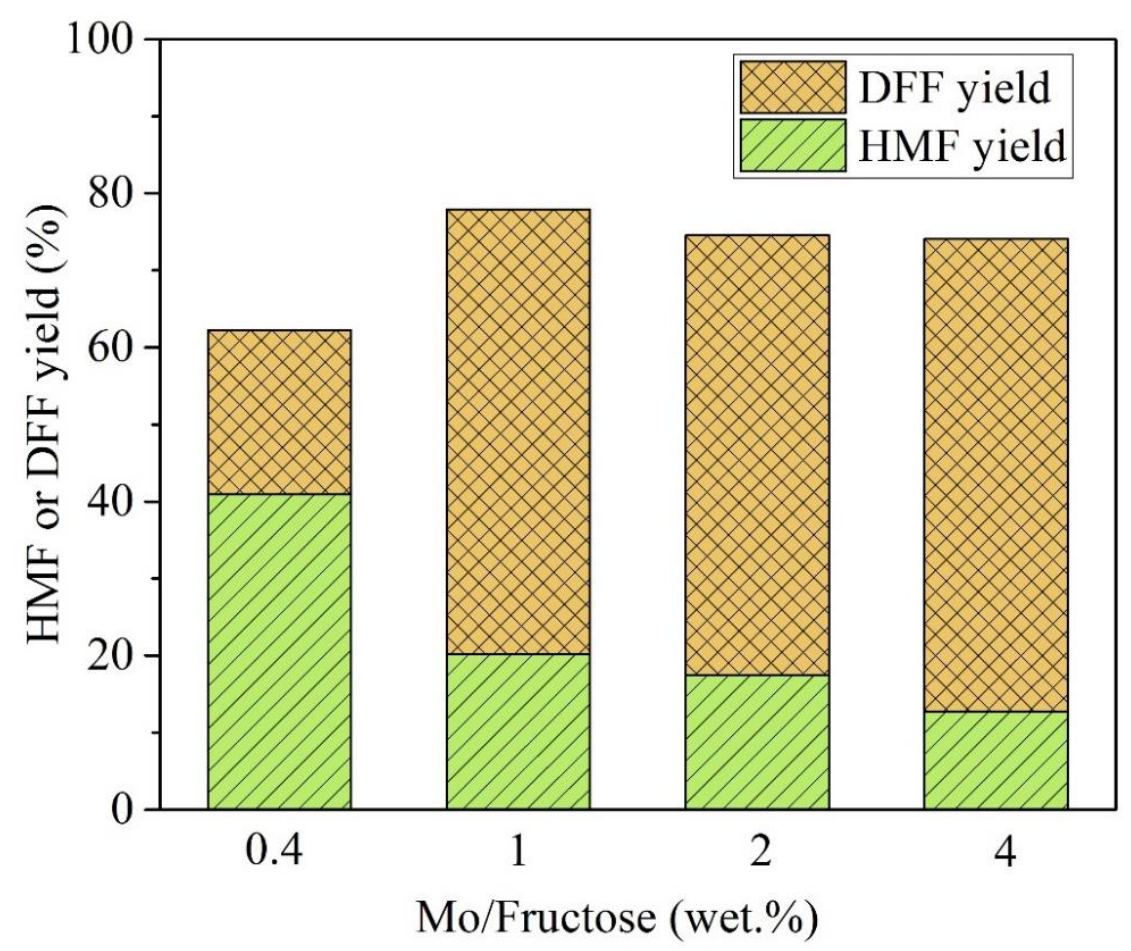

Fig. 6.10 Influence of ratio of Mo/fructose on the synthesis of HMF and DFF from fructose. Reaction conditions: CS-Mo-275 (30mg), DMSO (5mL), O $(20 \mathrm{~mL} / \mathrm{min}), 130^{\circ} \mathrm{C}, 18 \mathrm{~h}$

In terms of the final results, reaction temperature has a significate influence to the yield of the target product (Table 6.2). It has been proven that the dehydration of fructose to HMF ran very fast under the investigated temperature range and reached $100 \%$ conversion of fructose, turning the reaction into an 
aerobic oxidation reaction of HMF. Thus the temperature affects the final yield of DFF by affecting the HMF oxidation rate. For instance, 95.98 fructose conversion and 69.60 HMF yield were attained in $2 \mathrm{~h}$ at $110 \mathrm{C}$, while the DFF yield was only $1.44 \%$. This is because the rate of HMF oxidation is far slower than fructose dehydration at rate $110^{\circ} \mathrm{C}$, resulting in the accumulation of large amount of HMF in the reaction system. Nevertheless, when the reaction temperature raised to $150^{\circ} \mathrm{C}$, HMF yield of 34.34 and DFF yield of 38.02 were achieved with fructose conversion of $100 \%$ after the same reaction time period. What deserves to be noticed is that when the reaction temperature further increased to $165^{\circ} \mathrm{C}$, DFF yield could be as high as $77.85 \%$ in $2 \mathrm{~h}$ with thimbleful HMF residues.

Table 6.2 Influence of temperature and time on the synthesis of HMF and DFF from fructose

\begin{tabular}{|c|c|c|c|c|c|c|}
\hline Entry & $\begin{array}{l}\text { Mo/fructose } \\
\text { (wet.\%) }\end{array}$ & $\begin{array}{l}\text { Tim } \\
\mathrm{e}(\mathrm{h})\end{array}$ & $\begin{array}{l}\text { Temperat } \\
\text { ure }\left({ }^{\circ} \mathrm{C}\right)\end{array}$ & $\begin{array}{l}\text { Fructose } \\
\text { conv. }(\%)\end{array}$ & $\begin{array}{r}\text { HMF } \\
\text { yield }(\%)\end{array}$ & $\begin{array}{r}\text { DFF } \\
\text { yield }(\%)\end{array}$ \\
\hline 1 & 1 & 2 & 110 & 95.98 & 69.60 & 1.44 \\
\hline 2 & 1 & 72 & 110 & 100 & 10.31 & 50.81 \\
\hline 3 & 1 & 18 & 130 & 100 & 20.16 & 57.78 \\
\hline 4 & 1 & 2 & 150 & 100 & 34.34 & 2 \\
\hline 5 & 1 & 5 & 150 & 100 & 4.33 & 66.83 \\
\hline 6 & 1 & 2 & 165 & 100 & 0.8 & 77.85 \\
\hline
\end{tabular}

Reaction conditions: fructose (200mg), CS-Mo-275 (30mg), DMSO (5mL), $\mathrm{O}_{2}(20 \mathrm{~mL} / \mathrm{min})$

\subsection{Reusability of the catalyst}

Five-cycle experiments were conducted to study the reusability of Mo-CS275. The used catalyst was separated from the reaction mixture by filtration, washed with ethanol and DI water, followed by drying under vacuum at $45^{\circ} \mathrm{C}$ and 
calcining at $275^{\circ} \mathrm{C}$ for $3 \mathrm{~h}$ before the next run. Generally, the catalyst can run for long time and be reused without significant decreased of catalytic performance as shown in Fig. 6.10. In details, DFF yield decreased from $61.67 \%$ to $53.85 \%$ after the first run, then the descending of DFF yield developed slowly and the content of HMF increased slightly, while the fructose conversion kept at $100 \%$. $\mathrm{XRD}$ analysis shows that the XRD peaks of molybdenum trioxide species in the catalysis become very weak or disappear. According to the FESEM pictures in Fig. 6.11, the spherical morphology of the catalyst basically remained with slight scuffing and chipping. In addition, the TGA result showed that the molybdenum content of the catalyst dropped by $27 \%$ after the recycling test, suggesting the leaching of the active sites, which is the main reason for the changes of XRD pattern and catalytic activity.

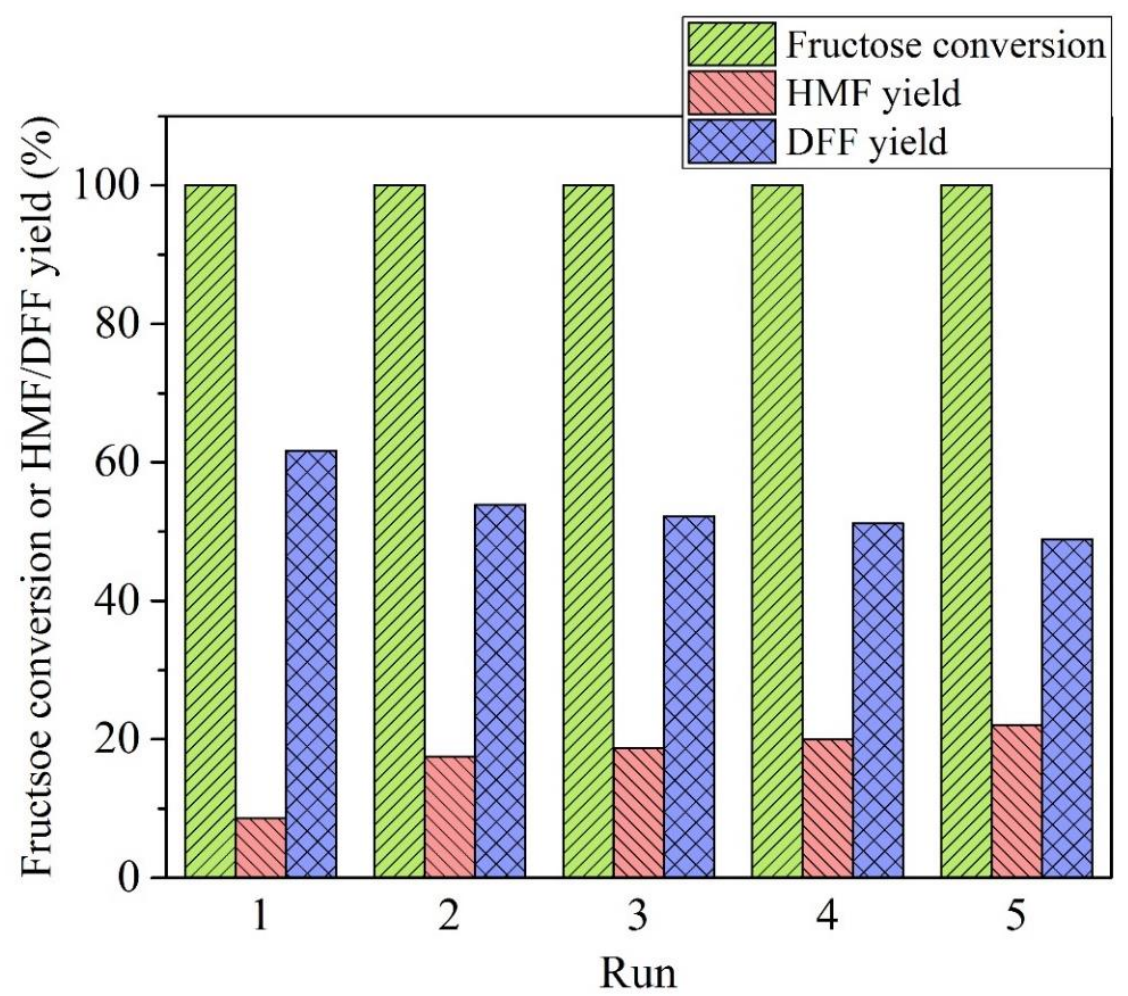

Fig. 6.11 Reusability test of CS-Mo-275. Reaction conditions: fructose (200mg), CS-Mo-275 (30mg), DMSO (5mL), O 2 (20mL/min), $155^{\circ} \mathrm{C}, 2 \mathrm{~h}$. 

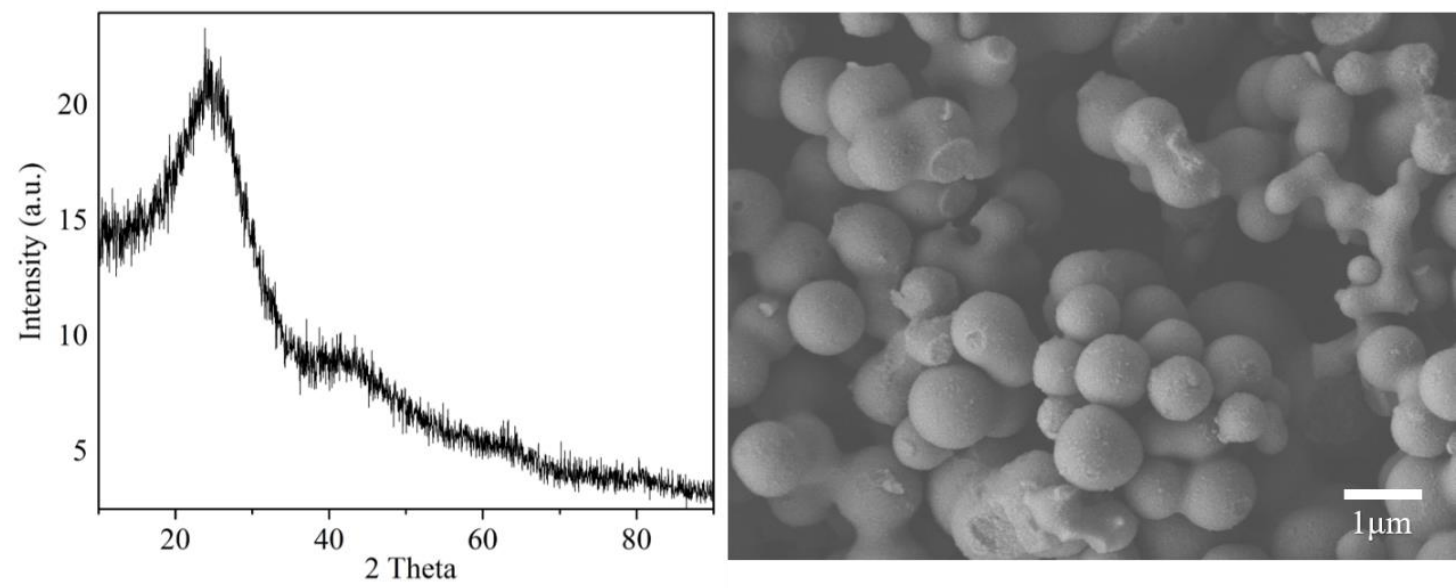

Fig. 6.12 XRD and FESEM picture of recovered Mo-CS-275

\subsection{Summary}

For a long time, people have got the idea of preparing catalysts with both acid sites and oxidative sites to apply in the one-pot conversion of fructose to DFF. In fact, it is quite challenging to find such kinds of catalysts which can catalyze the reaction and achieve a satisfactory result-not because the catalysts are too difficult to design and prepare, but because the conflicts between the function of the two kinds of active sites are difficult to deal with. Generally, organic acids such as formic acid, levulinic acid and humic acid will appear in the reaction system as by-products of the dehydration of fructose to HMF. These acid products will always be the poisoning reason of the catalyst which are usually used in the aerobic oxidation of HMF to DFF[409]. This issue has led to the limitation on use of catalysts such as $\mathrm{Pt} / \mathrm{C}, \mathrm{Pd} / \mathrm{C}, \mathrm{Pt} / \mathrm{TiO}_{2} / \mathrm{ZrO}_{2}$ and so on[386]. Later, a series of vanadium oxide catalysts were found to be efficient not only for the oxidation of pure HMF but also efficient for the in-situ oxidation of HMF generated from fructose. But new issue emerged during the application of vanadium oxide catalysts in transformation of fructose to DFF. Fructose was over oxidized and degraded to unwanted organic acid and insoluble polymeric furanic compounds due to the strong oxidative ability of the catalyst[410]. Therefore, the ideal catalyst should meet the following requirements: tolerance to all the organic by-products and water, mildly oxidize ability which can catalyze the aerobic 
oxidation of HMF instead of leading to rapid decomposition of fructose. Fortunately, the as-synthesized catalyst is such a catalyst which meets all the above requirement, therefore it can be successfully applied in the one-pot and one-step synthesis of DFF from fructose. The excellent catalytic performance of the catalyst is exactly attributed to the relative high acidity and moderate oxidative ability. The former catalyzes the dehydration of fructose to HMF, while the later acts on the aerobic oxidation of HMF, thus when these two factors affect together DFF can be obtained as a product directly from fructose. Under optimized conditions, $77.85 \%$ yield of DFF was obtained from fructose with MoCS-275 as the catalyst and bubbling oxygen as the oxidant in only $2 \mathrm{~h}$ at $165^{\circ} \mathrm{C}$. In addition, the catalyst could be separated and reused at least five cycles without significant loss in activity. 


\section{Chapter 7 Conclusions and Future Work}

\subsection{Conclusions}

In this study, series of carbon-based catalysts were synthesized and employed for the conversion of fructose to HMF and its derived chemicals. The investigated reactions have huge implication for the utilization of biomass materials. The catalysts were well characterized by advanced technologies in order to emerge the physical and chemical properties and their relationship with the catalytic performance. Reaction conditions were also optimized to achieve a highest possible yield of the target products.

Carbon materials have long been used as support materials because they can be chemically functionalized with various active groups and decorated with metallic nanoparticles. In this thesis, carbon spheres were chosen as the support materials and modified according to the requirements of the reactions. Compared with other carbon materials such as carbon nanotubes, graphene and active carbon, carbon spheres were prepared by a facile hydrothermal method at temperature lower than $200^{\circ} \mathrm{C}$, which is a more economic and greener synthesis method. Carbon spheres bearing $-\mathrm{SO}_{3} \mathrm{H}$ groups can be prepared by functionalizing the carbon materials with sulfuric acid. The as-synthesized catalyst is an insoluble Bronsted acid and showed good performance in the dehydration of fructose to HMF under mild conditions. $90 \%$ yield of HMF can be achieved in only $1.5 \mathrm{~h}$ at $160^{\circ} \mathrm{C}$. It worth to note that solvent play an important role in the fructose dehydration. On one hand, the $\mathrm{S}=\mathrm{O}$ structure in DMSO can catalyze the dehydration of fructose to HMF as a catalyst and on the other hand, DMSO can stay in coordination with the hydroxyl group and C1 carbon of HMF which can prevent the side reactions such as the rehydration of HMF to formic acid and levulinc acid.

Carbon supported metal oxide catalysts have been widely used in the transformation of biomass to fuels and chemicals. In Chapter 5, we described the 
synthesis of vanadium-embedded mesoporous carbon spheres and their application in the aerobic oxidation of HMF to DFF. As known to all, the surface area of hydrothermal synthesized carbon spheres is usually very small, which is not favorable for the fully contact between the reactant and active sites. To overcome this problem, the vanadium-embedded mesoporous carbon spheres were calcined under high temperature with limited oxygen to introduce porous structures in to the solid carbon spheres. As a result, the surface area of the carbon spheres increased dramatically as well as the catalytic activity. Under optimized reaction conditions, HMF conversion of $100 \%$ and DFF yield of more than $99 \%$ were achieved when only 2 wt.\% (V/HMF) catalyst was applied in the aerobic oxidation of HMF to DFF with DMSO as solvent at $120^{\circ} \mathrm{C}$ and oxygen bubbling at a flow rate of $20 \mathrm{~mL} / \mathrm{min}$ after $9 \mathrm{~h}$. The only weak point here is the little decline in the catalytic activity due to the leaching of the active sites during the recycling tests, which is a common problem for the vanadium oxide catalysts.

Since dehydration of fructose to HMF is an acid catalyzed reaction and the conversion of HMF to DFF is an aerobic oxidation reaction, people will naturally think it is very hopeful to synthesis DFF directly from fructose catalyzing by catalysts bearing both acid sites and oxidative sites. Actually, it is quite challenging to prepare such kinds of catalysts and exhibit satisfactory catalytic effect. One reason is that the by-products of the dehydration of fructose to HMF such as formic acid, levulinic acid and humic acid always invalid the oxidative active sites, terminating the reaction at the first step. The other reason is that most of the oxidative catalysts which is efficient in the aerobic oxidation of HMF to DFF can also oxidize fructose, leading to rapid decomposition of fructose and poor yield of DFF. Fortunately, molybdenum trioxide supported on carbon sphere as described in chapter 6 is an excellent catalyst with high acidity and moderate oxidative ability. Thus the catalysts can facilitate the dehydration of fructose to HMF on one hand, and will not degrade fructose to unwanted products. 
Under optimized conditions, $77.85 \%$ yield of DFF was obtained from fructose with Mo-CS-275 as the catalyst and bubbling oxygen as the oxidant in only $2 \mathrm{~h}$ at $165^{\circ} \mathrm{C}$. In addition, the catalyst could be separated and reused at least five cycles without significant loss in activity.

In conclusion, carbon materials based/supported catalysts are the one of the most achievable and economical catalysts for the conversion of biomass materials to value-added chemicals.

\subsection{Future Work}

Based upon the discoveries in the previous $\mathrm{PhD}$ work, we proposed some research topics which may be interesting and worthy of further work. In chapter 4, carbon sphere solid showed excellent catalytic performance in the dehydration of fructose to HMF. We noticed that the surface area of the carbon spheres is not very high and this may influence the fully utilization of the carbon materials. Therefore, mesoporous carbon sphere will be a better choice for the support of acid sites. While it is important to note, completely carbonized carbon materials are difficult to be sulfonated and grafted with high-density acid sites. Thus the processing temperature during the synthesis of mesoporous carbon spheres could not be too high. What is more, nitrogen doping is a common approach to change the physicochemical properties of carbon materials. Thus it deserves to be further investigated on the catalytic effect of nitrogen-doped mesoporous carbon spheres based solid acid for the dehydration of HMF.

In chapter 5, vanadium oxide was the active site for the aerobic oxidation of HMF to DFF. But its strong oxidizability can lead to the aerobic degradation of fructose to various by-products, which limited the use of it for the one-pot and one-step synthesis of DFF from fructose. However, bimetallic oxide consisting of vanadium and another metal with lower oxidizability maybe will help overcome this disadvantage. If the bimetallic oxide contains Lewis acid sites on its surface, then it could act as a bifuncitonal catalyst and will probably be active 
in both the dehydration of fructose to HMF and oxidation of HMF to DFF. In this way, it can be used in the direct transformation of fructose to DFF.

In chapter 6, carbon spheres supported molybdenum trioxide has proved to be an efficient catalyst for the one-step approach conversion of fructose to DFF. Actually, phosphomolybdic acid is an ideal catalyst with strong Bronsted acid sites and appropriate oxidize ability, except the fact that it is soluble in the reaction solvents and form a homogeneous reaction system which cause trouble in the recycle of catalyst. A solution is immobilizing the POM (phosphomolybdic acid) by preparing MOF (metal organic framework) encapsulated Kegginpolyoxometalate catalyst. In this way, the hybrid material can be used in the direct conversion of fructose to DFF and is easy to be separated from the reaction solution.

The above work plans are more focused on the improvement of the performance of the catalysts. Actually, the mentioned reaction can be extended to use cheaper carbohydrates as the starting materials. To produce glucose by catalytic hydrolysis of cellulose using carbon-based solid acid catalysts has attracted much attention, because the heterogeneous reaction systems can avoid disadvantages such as product separation, reactor corrosion and poor catalyst recyclability. The strength and density of the acid sites, the micropores of the carbon catalysts are the key factors which affect the catalytic performance. In addition, the isomerization of glucose to fructose is another important reaction which convert the not very sweet glucose to fructose, the sweetest natural sugars. Glucose is the most abundant monosaccharide available in nature and is the cheapest one. The combination of glucose isomerization with fructose dehydration can make it possible to synthesis DFF from glucose in a one-pot reaction, which can further lower the production cost of DFF. It can be clearly seen that the isomerization of glucose to fructose is a bridge reaction which connects the synthesis of HMF derived chemicals to the conversion of glucose 
and even cellulose. This progress is of great practical significance because it can greatly simplify the production process, reduce the energy consumption and lower the production cost.

\section{Reference}

[1] B.W. Ang, F. Zhang, A survey of index decomposition analysis in energy and environmental studies, Energy, 25 (2000) 1149-1176.

[2] N. Krichene, World crude oil and natural gas: a demand and supply model, Energy economics, 24 (2002) 557-576.

[3] E. Regnier, Oil and energy price volatility, Energy Economics, 29 (2007) 405427.

[4] A.K. Burnham, C.T. Stubblefield, J.H. Campbell, Effects of gas environment on mineral reactions in Colorado oil shale, Fuel, 59 (1980) 871-877.

[5] M. Kermani, A. Morshed, Carbon dioxide corrosion in oil and gas productionA compendium, Corrosion, 59 (2003) 659-683.

[6] M. Meinshausen, N. Meinshausen, W. Hare, S.C. Raper, K. Frieler, R. Knutti, D.J. Frame, M.R. Allen, Greenhouse-gas emission targets for limiting global warming to $2 \mathrm{C}$, Nature, 458 (2009) 1158-1162.

[7] P.M. Fearnside, Global warming and tropical land-use change: greenhouse gas emissions from biomass burning, decomposition and soils in forest conversion, shifting cultivation and secondary vegetation, Climatic change, 46 (2000) 115-158.

[8] J.-C. Sabonnadiere, Renewable energy technologies, John Wiley \& Sons 2010.

[9] T.J. Foxon, R. Gross, A. Chase, J. Howes, A. Arnall, D. Anderson, UK innovation systems for new and renewable energy technologies: drivers, barriers and systems failures, Energy policy, 33 (2005) 2123-2137.

[10] T.B. Johansson, Renewable energy: sources for fuels and electricity, Island press 1993.

[11] M.Z. Jacobson, M.A. Delucchi, Providing all global energy with wind, water, 
and solar power, Part I: Technologies, energy resources, quantities and areas of infrastructure, and materials, Energy Policy, 39 (2011) 1154-1169.

[12] J. Twidell, T. Weir, Renewable energy resources, Routledge2015.

[13] P. McKendry, Energy production from biomass (part 1): overview of biomass, Bioresource technology, 83 (2002) 37-46.

[14] N.S. Lewis, D.G. Nocera, Powering the planet: Chemical challenges in solar energy utilization, Proceedings of the National Academy of Sciences, 103 (2006) $15729-15735$.

[15] R. Saxena, D. Adhikari, H. Goyal, Biomass-based energy fuel through biochemical routes: a review, Renewable and Sustainable Energy Reviews, 13 (2009) 167-178.

[16] S. Rehman, M.A. Bader, S.A. Al-Moallem, Cost of solar energy generated using PV panels, Renewable and Sustainable Energy Reviews, 11 (2007) 18431857.

[17] E.S. Hrayshat, Viability of solar photovoltaics as an electricity generation source for Jordan, International Journal of Sustainable Engineering, 2 (2009) 6777.

[18] M.O. Andreae, Biomass burning: its history, use, and distribution and its impact on environmental quality and global climate, Global biomass burning: Atmospheric, climatic and biospheric implications, (1991) 3-21.

[19] S. Whitlow, P. Mayewski, J. Dibb, G. Holdsworth, M. Twickler, An ice-corebased record of biomass burning in the Arctic and Subarctic, 1750-1980, Tellus B, 46 (1994) 234-242.

[20] S.N. Naik, V.V. Goud, P.K. Rout, A.K. Dalai, Production of first and second generation biofuels: a comprehensive review, Renewable and Sustainable Energy Reviews, 14 (2010) 578-597.

[21] P.M. Schenk, S.R. Thomas-Hall, E. Stephens, U.C. Marx, J.H. Mussgnug, C. Posten, O. Kruse, B. Hankamer, Second generation biofuels: high-efficiency 
microalgae for biodiesel production, Bioenergy research, 1 (2008) 20-43.

[22] R. Chandra, H. Takeuchi, T. Hasegawa, Methane production from lignocellulosic agricultural crop wastes: A review in context to second generation of biofuel production, Renewable and Sustainable Energy Reviews, 16 (2012) $1462-1476$.

[23] S. Dasappa, P. Paul, H. Mukunda, N. Rajan, G. Sridhar, H. Sridhar, Biomass gasification technology-a route to meet energy needs, Current Science, 87 (2004) 908-916.

[24] M.J. Prins, K.J. Ptasinski, F.J. Janssen, More efficient biomass gasification via torrefaction, Energy, 31 (2006) 3458-3470.

[25] N. Panwar, S. Kaushik, S. Kothari, Role of renewable energy sources in environmental protection: a review, Renewable and Sustainable Energy Reviews, 15 (2011) 1513-1524.

[26] L.F. Albright, J.C. Marek, Mechanistic model for formation of coke in pyrolysis units producing ethylene, Industrial \& engineering chemistry research, 27 (1988) 755-759.

[27] S.S. Toor, L. Rosendahl, A. Rudolf, Hydrothermal liquefaction of biomass: a review of subcritical water technologies, Energy, 36 (2011) 2328-2342.

[28] N. Mosier, C. Wyman, B. Dale, R. Elander, Y. Lee, M. Holtzapple, M. Ladisch, Features of promising technologies for pretreatment of lignocellulosic biomass, Bioresource technology, 96 (2005) 673-686.

[29] S.M. Kotay, D. Das, Biohydrogen as a renewable energy resourceprospects and potentials, International Journal of Hydrogen Energy, 33 (2008) $258-263$

[30] J.J. Bozell, L. Moens, D. Elliott, Y. Wang, G. Neuenscwander, S. Fitzpatrick, R. Bilski, J. Jarnefeld, Production of levulinic acid and use as a platform chemical for derived products, Resources, Conservation and Recycling, 28 (2000) 227-239. [31] D.M. Alonso, J.Q. Bond, J.A. Dumesic, Catalytic conversion of biomass to 
biofuels, Green Chemistry, 12 (2010) 1493-1513.

[32] D. Beall, L. Ingram, Genetic engineering of soft-rot bacteria for ethanol production from lignocellulose, Journal of industrial microbiology, 11 (1993) $151-155$.

[33] J. Zaldivar, J. Nielsen, L. Olsson, Fuel ethanol production from lignocellulose: a challenge for metabolic engineering and process integration, Applied microbiology and biotechnology, 56 (2001) 17-34.

[34] B. Kuster, 5-Hydroxymethylfurfural (HMF). A review focussing on its manufacture, Starch-Stärke, 42 (1990) 314-321.

[35] M.L. Ribeiro, U. Schuchardt, Cooperative effect of cobalt acetylacetonate and silica in the catalytic cyclization and oxidation of fructose to 2, 5furandicarboxylic acid, Catalysis Communications, 4 (2003) 83-86.

[36] W. Partenheimer, V.V. Grushin, Synthesis of 2, 5-Diformylfuran and Furan2, 5-Dicarboxylic Acid by Catalytic Air-Oxidation of 5-Hydroxymethylfurfural. Unexpectedly Selective Aerobic Oxidation of Benzyl Alcohol to Benzaldehyde with Metal= Bromide Catalysts, Advanced Synthesis \& Catalysis, 343 (2001) $102-111$.

[37] A. Al Baradii, K. Kokoh, H. Huser, C. Lamy, J.-M. Léger, Selective electrocatalytic oxidation of 2, 5-dihydroxymethylfuran in aqueous medium: a chromatographic analysis of the reaction products, Electrochimica acta, 44 (1999) $2779-2787$.

[38] C. Marisa, R. Marotta, A. Roberto, C. Vincenzo, Production of 5-hydroxy4-keto-2-pentenoic acid by photo-oxidation of 5-hydroxymethylfurfural with singlet oxygen: A kinetic investigation, Journal of Photochemistry and Photobiology A: Chemistry, 210 (2010) 69-76.

[39] H. Heeres, R. Handana, D. Chunai, C.B. Rasrendra, B. Girisuta, H.J. Heeres, Combined dehydration/(transfer)-hydrogenation of C6-sugars (D-glucose and Dfructose) to $\gamma$-valerolactone using ruthenium catalysts, Green Chemistry, 11 
(2009) 1247-1255.

[40] X. Tong, Y. Ma, Y. Li, Biomass into chemicals: conversion of sugars to furan derivatives by catalytic processes, Applied Catalysis A: General, 385 (2010) 113.

[41] A. Corma, S. Iborra, A. Velty, Chemical routes for the transformation of biomass into chemicals, Chemical reviews, 107 (2007) 2411-2502.

[42] D.S. van Es, S. Marinkovic, X. Oduber, B. Estrine, Use of Furandicarboxylic Acid and Its Decyl Ester as Additives in the Fischer's Glycosylation of Decanol by d-Glucose: Physicochemical Properties of the Surfactant Compositions Obtained, Journal of Surfactants and Detergents, 16 (2013) 147-154.

[43] T. Xiang, X. Liu, P. Yi, M. Guo, Y. Chen, C. Wesdemiotis, J. Xu, Y. Pang, Schiff base polymers derived from 2, 5-diformylfuran, Polymer International, 62 (2013) 1517-1523.

[44] A.S. Amarasekara, D. Green, L.D. Williams, Renewable resources based polymers: Synthesis and characterization of 2, 5-diformylfuran-urea resin, European Polymer Journal, 45 (2009) 595-598.

[45] A. Robertson, The early history of catalysis, Platinum Metals Rev, 19 (1975) 64-69.

[46] G. Yadav, Insight into green phase transfer catalysis, Topics in catalysis, 29 (2004) 145-161.

[47] A.A. Rosatella, S.P. Simeonov, R.F. Frade, C.A. Afonso, 5Hydroxymethylfurfural (HMF) as a building block platform: Biological properties, synthesis and synthetic applications, Green Chemistry, 13 (2011) 754793.

[48] Y. Guo, J. Chen, Bicomponent Assembly of VO2 and PolyanilineFunctionalized Carbon Nanotubes for the Selective Oxidation of Biomass-Based 5-Hydroxymethylfurfural to 2, 5-Diformylfuran, ChemPlusChem, 80 (2015) $1760-1768$. 
[49] Y. Zhu, X. Liu, M. Shen, Y. Xia, M. Lu, Nano-ruthenium particles supported on a core-shell shuttle: As an efficient lipophilic catalyst for the aerobic oxidation of biomass-derived 5-(Hydroxymethyl) furfural, Catalysis Communications, 63 (2015) 21-25.

[50] J.N. Chheda, G.W. Huber, J.A. Dumesic, Liquid-phase catalytic processing of biomass-derived oxygenated hydrocarbons to fuels and chemicals, Angewandte Chemie International Edition, 46 (2007) 7164-7183.

[51] J. Mitra, X. Zhou, T. Rauchfuss, Pd/C-catalyzed reactions of HMF: decarbonylation, hydrogenation, and hydrogenolysis, Green Chemistry, 17 (2015) 307-313.

[52] J. Artz, S. Mallmann, R. Palkovits, Selective Aerobic Oxidation of HMF to 2, 5-Diformylfuran on Covalent Triazine Frameworks-Supported Ru Catalysts, ChemSusChem, 8 (2015) 672-679.

[53] D.M. Alonso, J.M.R. Gallo, M.A. Mellmer, S.G. Wettstein, J.A. Dumesic, Direct conversion of cellulose to levulinic acid and gamma-valerolactone using solid acid catalysts, Catalysis Science \& Technology, 3 (2013) 927-931.

[54] Q. Bao, K. Qiao, D. Tomida, C. Yokoyama, Preparation of 5hydroymethylfurfural by dehydration of fructose in the presence of acidic ionic liquid, Catalysis Communications, 9 (2008) 1383-1388.

[55] C. Moreau, A. Finiels, L. Vanoye, Dehydration of fructose and sucrose into 5-hydroxymethylfurfural in the presence of 1-H-3-methyl imidazolium chloride acting both as solvent and catalyst, Journal of Molecular Catalysis A: Chemical, 253 (2006) 165-169.

[56] Y. Su, H.M. Brown, X. Huang, X.-d. Zhou, J.E. Amonette, Z.C. Zhang, Single-step conversion of cellulose to 5-hydroxymethylfurfural (HMF), a versatile platform chemical, Applied Catalysis A: General, 361 (2009) 117-122. [57] J.C. Serrano-Ruiz, R. Luque, A. Sepulveda-Escribano, Transformations of biomass-derived platform molecules: from high added-value chemicals to fuels 
via aqueous-phase processing, Chemical Society Reviews, 40 (2011) 5266-5281. [58] Z. Zhang, Z.K. Zhao, Microwave-assisted conversion of lignocellulosic biomass into furans in ionic liquid, Bioresource technology, 101 (2010) 11111114.

[59] E. Capuano, V. Fogliano, Acrylamide and 5-hydroxymethylfurfural (HMF): A review on metabolism, toxicity, occurrence in food and mitigation strategies, LWT-Food Science and Technology, 44 (2011) 793-810.

[60] A. Cukalovic, C.V. Stevens, Production of biobased HMF derivatives by reductive amination, Green Chemistry, 12 (2010) 1201-1206.

[61] J. Kiermayer, A derivative of furfuraldehyde from laevulose, Chem. Ztg, 19 (1895) 1003-1006.

[62] M.S. Feather, J.F. Harris, Dehydration reactions of carbohydrates, Advances in carbohydrate chemistry and biochemistry, 28 (1973) 161-224.

[63] L. Cottier, G. Descotes, 5-(Hydroxymethyl) furfural Syntheses and Chemical Transformations, ChemInform, 25 (1994).

[64] J.S. Kim, Y. Lee, R.W. Torget, Cellulose hydrolysis under extremely low sulfuric acid and high-temperature conditions, Applied biochemistry and biotechnology, 91 (2001) 331-340.

[65] D. Ray, N. Mittal, W.-J. Chung, Phosphorous pentoxide mediated synthesis of 5-HMF in ionic liquid at low temperature, Carbohydrate research, 346 (2011) $2145-2148$.

[66] Y. Takeuchi, F. Jin, K. Tohji, H. Enomoto, Acid catalytic hydrothermal conversion of carbohydrate biomass into useful substances, Journal of Materials Science, 43 (2008) 2472-2475.

[67] S. Hu, Z. Zhang, Y. Zhou, J. Song, H. Fan, B. Han, Direct conversion of inulin to 5-hydroxymethylfurfural in biorenewable ionic liquids, Green Chemistry, 11 (2009) 873-877.

[68] C. Chang, P. Cen, X. Ma, Levulinic acid production from wheat straw, 
Bioresource technology, 98 (2007) 1448-1453.

[69] F. Salak Asghari, H. Yoshida, Acid-catalyzed production of 5hydroxymethyl furfural from D-fructose in subcritical water, Industrial \& Engineering Chemistry Research, 45 (2006) 2163-2173.

[70] B.M. Kabyemela, T. Adschiri, R.M. Malaluan, K. Arai, Glucose and fructose decomposition in subcritical and supercritical water: detailed reaction pathway, mechanisms, and kinetics, Industrial \& Engineering Chemistry Research, 38 (1999) 2888-2895.

[71] J.E. Stone, M.J. Blundell, A MICROMETHOD FOR THE DETERMINATION OF SUGARS, Canadian Journal of Research, 28b (1950) 676-682.

[72] M. Schön, M. Schnürch, M.D. Mihovilovic, Application of continuous flow and alternative energy devices for 5-hydroxymethylfurfural production, Molecular diversity, 15 (2011) 639-643.

[73] X. Tong, M. Li, N. Yan, Y. Ma, P.J. Dyson, Y. Li, Defunctionalization of fructose and sucrose: Iron-catalyzed production of 5-hydroxymethylfurfural from fructose and sucrose, Catalysis today, 175 (2011) 524-527.

[74] Z. Zhang, B. Liu, Z.K. Zhao, Conversion of fructose into 5-HMF catalyzed by $\mathrm{GeCl} 4$ in $\mathrm{DMSO}$ and [Bmim] $\mathrm{Cl}$ system at room temperature, Carbohydrate polymers, 88 (2012) 891-895.

[75] Z. Wei, Y. Liu, D. Thushara, Q. Ren, Entrainer-intensified vacuum reactive distillation process for the separation of 5-hydroxylmethylfurfural from the dehydration of carbohydrates catalyzed by a metal salt-ionic liquid, Green Chemistry, 14 (2012) 1220-1226.

[76] F. Wang, A.-W. Shi, X.-X. Qin, C.-L. Liu, W.-S. Dong, Dehydration of fructose to 5-hydroxymethylfurfural by rare earth metal trifluoromethanesulfonates in organic solvents, Carbohydrate research, 346 (2011) 982-985. 
[77] J.-D. Chen, B.F. Kuster, K. Van Der Wiele, Preparation of 5hydroxymethylfurfural via fructose acetonides in ethylene glycol dimethyl ether, Biomass and Bioenergy, 1 (1991) 217-223.

[78] C. Moreau, R. Durand, F. Aliès, M. Cotillon, T. Frutz, M.-A. Théoleyre, Hydrolysis of sucrose in the presence of H-form zeolites, Industrial Crops and Products, 11 (2000) 237-242.

[79] C. Carlini, P. Patrono, A.M.R. Galletti, G. Sbrana, Heterogeneous catalysts based on vanadyl phosphate for fructose dehydration to 5-hydroxymethyl-2furaldehyde, Applied Catalysis A: General, 275 (2004) 111-118.

[80] T. Armaroli, G. Busca, C. Carlini, M. Giuttari, A.M.R. Galletti, G. Sbrana, Acid sites characterization of niobium phosphate catalysts and their activity in fructose dehydration to 5-hydroxymethyl-2-furaldehyde, Journal of Molecular Catalysis A: Chemical, 151 (2000) 233-243.

[81] P. Carniti, A. Gervasini, S. Biella, A. Auroux, Niobic acid and niobium phosphate as highly acidic viable catalysts in aqueous medium: Fructose dehydration reaction, Catalysis Today, 118 (2006) 373-378.

[82] C. Carlini, M. Giuttari, A.M.R. Galletti, G. Sbrana, T. Armaroli, G. Busca, Selective saccharides dehydration to 5-hydroxymethyl-2-furaldehyde by heterogeneous niobium catalysts, Applied Catalysis A: General, 183 (1999) 295302.

[83] F. Benvenuti, C. Carlini, P. Patrono, A.M.R. Galletti, G. Sbrana, M.A. Massucci, P. Galli, Heterogeneous zirconium and titanium catalysts for the selective synthesis of 5-hydroxymethyl-2-furaldehyde from carbohydrates, Applied Catalysis A: General, 193 (2000) 147-153.

[84] H. Yan, Y. Yang, D. Tong, X. Xiang, C. Hu, Catalytic conversion of glucose to 5-hydroxymethylfurfural over SO 4 2-/ZrO 2 and SO 4 2-/ZrO 2-Al $2 \mathrm{O} 3$ solid acid catalysts, Catalysis Communications, 10 (2009) 1558-1563.

[85] M. Watanabe, Y. Aizawa, T. Iida, R. Nishimura, H. Inomata, Catalytic 
glucose and fructose conversions with $\mathrm{TiO} 2$ and $\mathrm{ZrO} 2$ in water at $473 \mathrm{~K}$ : Relationship between reactivity and acid-base property determined by TPD measurement, Applied Catalysis A: General, 295 (2005) 150-156.

[86] X. Qi, M. Watanabe, T.M. Aida, R.L. Smith, Catalytical conversion of fructose and glucose into 5-hydroxymethylfurfural in hot compressed water by microwave heating, Catalysis Communications, 9 (2008) 2244-2249.

[87] B. Saha, S. De, M. Fan, $\mathrm{Zr}(\mathrm{O}) \mathrm{Cl} 2$ catalyst for selective conversion of biorenewable carbohydrates and biopolymers to biofuel precursor 5hydroxymethylfurfural in aqueous medium, Fuel, 111 (2013) 598-605.

[88] X. Qi, M. Watanabe, T.M. Aida, R.L. Smith, Sulfated zirconia as a solid acid catalyst for the dehydration of fructose to 5-hydroxymethylfurfural, Catalysis Communications, 10 (2009) 1771-1775.

[89] A.H. Jadhav, H. Kim, I.T. Hwang, An efficient and heterogeneous recyclable silicotungstic acid with modified acid sites as a catalyst for conversion of fructose and sucrose into 5-hydroxymethylfurfural in superheated water, Bioresource Technology, 132 (2013) 342-350.

[90] J.N. Chheda, J.A. Dumesic, An overview of dehydration, aldol-condensation and hydrogenation processes for production of liquid alkanes from biomassderived carbohydrates, Catalysis Today, 123 (2007) 59-70.

[91] C. Lansalot-Matras, C. Moreau, Dehydration of fructose into 5hydroxymethylfurfural in the presence of ionic liquids, Catalysis Communications, 4 (2003) 517-520.

[92] H.H. Szmant, D.D. Chundury, The preparation of 5hydroxymethylfurfuraldehyde from high fructose corn syrup and other carbohydrates, Journal of Chemical Technology and Biotechnology, 31 (1981) $135-145$.

[93] J.N. Chheda, Y. Román-Leshkov, J.A. Dumesic, Production of 5hydroxymethylfurfural and furfural by dehydration of biomass-derived mono- 
and poly-saccharides, Green Chemistry, 9 (2007) 342-350.

[94] K.-i. Seri, Y. Inoue, H. Ishida, Highly Efficient Catalytic Activity of Lanthanide (III) Ions for Conversion of Saccharides to 5-Hydroxymethyl-2furfural in Organic Solvents, Chemistry Letters, (2000) 22-23.

[95] L. Lai, Y. Zhang, The Production of 5-Hydroxymethylfurfural from Fructose in Isopropyl Alcohol: A Green and Efficient System, ChemSusChem, 4 (2011) $1745-1748$.

[96] Q. Cao, X. Guo, S. Yao, J. Guan, X. Wang, X. Mu, D. Zhang, Conversion of hexose into 5-hydroxymethylfurfural in imidazolium ionic liquids with and without a catalyst, Carbohydrate research, 346 (2011) 956-959.

[97] R.M. Musau, R.M. Munavu, The preparation of 5-hydroxymethyl-2furaldehyde (HMF) from d-fructose in the presence of DMSO, Biomass, 13 (1987) 67-74.

[98] C. Fayet, J. Gelas, Nouvelle methode de preparation du 5-hydroxymethyl2-furaldehyde par action de sels d'ammonium ou d'immonium sur les mono-, oligo-et poly-saccharides. acces direct aux 5-halogenomethyl-2-furaldehydes, Carbohydrate research, 122 (1983) 59-68.

[99] C. Li, Z.K. Zhao, A. Wang, M. Zheng, T. Zhang, Production of 5hydroxymethylfurfural in ionic liquids under high fructose concentration conditions, Carbohydrate research, 345 (2010) 1846-1850.

[100] X. Tong, Y. Li, Efficient and Selective Dehydration of Fructose to 5Hydroxymethylfurfural Catalyzed by Brønsted-Acidic Ionic Liquids, ChemSusChem, 3 (2010) 350-355.

[101] A.H. Jadhav, H. Kim, I.T. Hwang, Efficient selective dehydration of fructose and sucrose into 5-hydroxymethylfurfural (HMF) using dicationic room temperature ionic liquids as a catalyst, Catalysis Communications, 21 (2012) 96103.

[102] A.H. Jadhav, H. Kim, A mild, efficient, and selective deprotection of tert- 
butyldimethylsilyl (TBDMS) ethers using dicationic ionic liquid as a catalyst, Tetrahedron Letters, 53 (2012) 5338-5342.

[103] L. Hu, G. Zhao, W. Hao, X. Tang, Y. Sun, L. Lin, S. Liu, Catalytic conversion of biomass-derived carbohydrates into fuels and chemicals via furanic aldehydes, RSC Advances, 2 (2012) 11184-11206.

[104] Y. Román-Leshkov, J.N. Chheda, J.A. Dumesic, Phase modifiers promote efficient production of hydroxymethylfurfural from fructose, Science, 312 (2006) 1933-1937.

[105] J.C. van der Waal, E. de Jong, R. Luque, A. Balu, Chemocatalytic Processes for the Production of Bio-Based Chemicals from Carbohydrates, CRC Press: Boca Raton2013.

[106] P. Rivalier, J. Duhamet, C. Moreau, R. Durand, Development of a continuous catalytic heterogeneous column reactor with simultaneous extraction of an intermediate product by an organic solvent circulating in countercurrent manner with the aqueous phase, Catalysis today, 24 (1995) 165-171.

[107] C. Moreau, R. Durand, S. Razigade, J. Duhamet, P. Faugeras, P. Rivalier, P. Ros, G. Avignon, Dehydration of fructose to 5-hydroxymethylfurfural over Hmordenites, Applied Catalysis A: General, 145 (1996) 211-224.

[108] A.J. Crisci, M.H. Tucker, M.-Y. Lee, S.G. Jang, J.A. Dumesic, S.L. Scott, Acid-functionalized SBA-15-type silica catalysts for carbohydrate dehydration, Acs Catalysis, 1 (2011) 719-728.

[109] F. Yang, Q. Liu, X. Bai, Y. Du, Conversion of biomass into 5hydroxymethylfurfural using solid acid catalyst, Bioresource technology, 102 (2011) 3424-3429.

[110] C. Fan, H. Guan, H. Zhang, J. Wang, S. Wang, X. Wang, Conversion of fructose and glucose into 5-hydroxymethylfurfural catalyzed by a solid heteropolyacid salt, Biomass and bioenergy, 35 (2011) 2659-2665.

[111] Q. Zhao, L. Wang, S. Zhao, X. Wang, S. Wang, High selective production 
of 5-hydroymethylfurfural from fructose by a solid heteropolyacid catalyst, Fuel, 90 (2011) 2289-2293.

[112] A.C. Cope, Production and recovery of furans, Google Patents, 1959.

[113] L. Rigal, A. Gaset, Direct preparation of 5-hydroxymethyl-2furancarboxaldehyde from polyholosides: a chemical valorisation of the Jerusalem artichoke (Helianthus tuberosus L.), Biomass, 3 (1983) 151-163.

[114] Y. Pagán-Torres, T. Wang, J. Gallo, B. Shanks, ACS Catal. 2012, 2, 930934.(d) Chheda, JN; Román-Leshkov, Y.; Dumesic, JA, Green Chem, 9 (2007) $342-350$.

[115] Y. Yang, C.-w. Hu, M.M. Abu-Omar, Conversion of carbohydrates and lignocellulosic biomass into 5-hydroxymethylfurfural using $\mathrm{AlCl} 3 \cdot 6 \mathrm{H} 2 \mathrm{O}$ catalyst in a biphasic solvent system, Green Chemistry, 14 (2012) 509-513.

[116] V. Degirmenci, E.A. Pidko, P.C. Magusin, E.J. Hensen, Towards a Selective Heterogeneous Catalyst for Glucose Dehydration to 5-Hydroxymethylfurfural in Water: $\mathrm{CrCl} 2$ Catalysis in a Thin Immobilized Ionic Liquid Layer, ChemCatChem, 3 (2011) 969-972.

[117] Y. Román-Leshkov, J.A. Dumesic, Solvent effects on fructose dehydration to 5-hydroxymethylfurfural in biphasic systems saturated with inorganic salts, Topics in Catalysis, 52 (2009) 297-303.

[118] J. Lewkowski, Synthesis, chemistry and applications of 5-hydroxymethylfurfural and its derivatives, (2001).

[119] T. Werpy, G. Petersen, A. Aden, J. Bozell, J. Holladay, J. White, A. Manheim, D. Eliot, L. Lasure, S. Jones, Top value added chemicals from biomass. Volume 1-Results of screening for potential candidates from sugars and synthesis gas, DTIC Document, 2004.

[120] T. Reichstein, Notiz über 5-Oxymethyl-furfurol, Helvetica Chimica Acta, 9 (1926) 1066-1068.

[121] Y.Y. Gorbanev, S.K. Klitgaard, J.M. Woodley, C.H. Christensen, A. 
Riisager, Gold-Catalyzed Aerobic Oxidation of 5-Hydroxymethylfurfural in Water at Ambient Temperature, ChemSusChem, 2 (2009) 672-675.

[122] S.E. Davis, L.R. Houk, E.C. Tamargo, A.K. Datye, R.J. Davis, Oxidation of 5-hydroxymethylfurfural over supported $\mathrm{Pt}, \mathrm{Pd}$ and $\mathrm{Au}$ catalysts, Catalysis today, 160 (2011) 55-60.

[123] M. Van Deurzen, F. Van Rantwijk, R. Sheldon, Chloroperoxidase-catalyzed oxidation of 5-hydroxymethylfurfural, Journal of carbohydrate chemistry, 16 (1997) 299-309.

[124] A. Gandini, M.N. Belgacem, Furans in polymer chemistry, Progress in Polymer Science, 22 (1997) 1203-1379.

[125] R. Sheldon, Heterogeneous catalytic oxidation and fine chemicals, Studies in Surface Science and Catalysis, 59 (1991) 33-54.

[126] L. Cottier, G. Descotes, J. Lewkowski, R. Skowronski, Oxidation of 5hydroxymethylfurfural under sonochemical conditions, Polish journal of chemistry, 68 (1994) 693-698.

[127] L. Cottier, G. Descotes, E. Viollet, J. Lewkowski, R. Skowroñski, Oxidation of 5-hydroxymethylfurfural and derivatives to furanaldehydes with 2, 2, 6, 6-tetramethylpiperidine oxide radical-co-oxidant pairs, Journal of heterocyclic chemistry, 32 (1995) 927-930.

[128] L. Cottier, G. Descotes, J. Lewkowski, R. Skowroñski, ultrasonically accelerated syntheses of furan-24-dicarbaldehyde from 5-hydroxymethyl-2furfural, Organic preparations and procedures international, 27 (1995) 564-566. [129] C. Moreau, R. Durand, C. Pourcheron, D. Tichit, Selective oxidation of 5hydroxymethylfurfural to 2, 5-furan-dicarboxaldehyde in the presence of titania supported vanadia catalysts, Studies in Surface Science and Catalysis, 108 (1997) $399-406$.

[130] P. Verdeguer, N. Merat, A. Gaset, Oxydation catalytique du HMF en acide 2, 5-furane dicarboxylique, Journal of molecular catalysis, 85 (1993) 327-344. 
[131] C. Carlini, P. Patrono, A.M.R. Galletti, G. Sbrana, V. Zima, Selective oxidation of 5-hydroxymethyl-2-furaldehyde to furan-2, 5-dicarboxaldehyde by catalytic systems based on vanadyl phosphate, Applied Catalysis A: General, 289 (2005) 197-204.

[132] A.S. Amarasekara, D. Green, E. McMillan, Efficient oxidation of 5hydroxymethylfurfural to 2, 5-diformylfuran using Mn (III)-salen catalysts, Catalysis Communications, 9 (2008) 286-288.

[133] M.A. Lilga, R.T. Hallen, M. Gray, Production of oxidized derivatives of 5hydroxymethylfurfural (HMF), Topics in Catalysis, 53 (2010) 1264-1269.

[134] N. Jacquel, R. Saint-Loup, J.-P. Pascault, A. Rousseau, F. Fenouillot, Biobased alternatives in the synthesis of aliphatic-aromatic polyesters dedicated to biodegradable film applications, Polymer, 59 (2015) 234-242.

[135] C.H. Wilsens, N.J. Wullems, E. Gubbels, Y. Yao, S. Rastogi, B.A. Noordover, Synthesis, kinetics, and characterization of bio-based thermosets obtained through polymerization of a 2, 5-furandicarboxylic acid-based bis (2oxazoline) with sebacic acid, Polymer Chemistry, 6 (2015) 2707-2716.

[136] A. Eerhart, A. Faaij, M.K. Patel, Replacing fossil based PET with biobased PEF; process analysis, energy and GHG balance, Energy \& Environmental Science, 5 (2012) 6407-6422.

[137] G. Grabowski, J. Lewkowski, R. Skowroński, The electrochemical oxidation of 5-hydroxymethylfurfural with the nickel oxide/hydroxide electrode, Electrochimica Acta, 36 (1991) 1995.

[138] K.R. Vuyyuru, P. Strasser, Oxidation of biomass derived 5hydroxymethylfurfural using heterogeneous and electrochemical catalysis, Catalysis today, 195 (2012) 144-154.

[139] D.J. Chadderdon, L. Xin, J. Qi, Y. Qiu, P. Krishna, K.L. More, W. Li, Electrocatalytic oxidation of 5-hydroxymethylfurfural to 2, 5-furandicarboxylic acid on supported Au and Pd bimetallic nanoparticles, Green Chemistry, 16 (2014) 
$3778-3786$.

[140] S. Morikawa, Synthesis of 2, 5-furandicarboxaldehyde from 5hydroxymethylfurfural, Noguchi Kenkyusho Jiho, 22 (1979) 20-27.

[141] M. Krystof, M. Pérez-Sánchez, P. Domínguez de María, Lipase-Mediated Selective Oxidation of Furfural and 5-Hydroxymethylfurfural, ChemSusChem, 6 (2013) 826-830.

[142] W.P. Dijkman, D.E. Groothuis, M.W. Fraaije, Enzyme-Catalyzed Oxidation of 5-Hydroxymethylfurfural to Furan-2, 5-dicarboxylic Acid, Angewandte Chemie International Edition, 53 (2014) 6515-6518.

[143] W.P. Dijkman, C. Binda, M.W. Fraaije, A. Mattevi, Structure-based enzyme tailoring of 5-hydroxymethylfurfural oxidase, ACS Catalysis, 5 (2015) 18331839.

[144] G.A. Halliday, R.J. Young, V.V. Grushin, One-pot, two-step, practical catalytic synthesis of 2, 5-diformylfuran from fructose, Organic letters, 5 (2003) 2003-2005.

[145] C. Carlini, P. Patrono, A.M.R. Galletti, G. Sbrana, V. Zima, Selective oxidation of 5-hydroxymethyl-2-furaldehyde to furan-2,5-dicarboxaldehyde by catalytic systems based on vanadyl phosphate, Applied Catalysis A: General, 289 (2005) 197-204.

[146] S. Roy Goswami, M.J. Dumont, V. Raghavan, Starch to value added biochemicals, Starch-Stärke, (2015).

[147] Z.-Z. Yang, J. Deng, T. Pan, Q.-X. Guo, Y. Fu, A one-pot approach for conversion of fructose to 2, 5-diformylfuran by combination of Fe $3 \mathrm{O}$ 4-SBASO 3 H and K-OMS-2, Green Chemistry, 14 (2012) 2986-2989.

[148] A. Takagaki, M. Takahashi, S. Nishimura, K. Ebitani, One-pot synthesis of 2, 5-diformylfuran from carbohydrate derivatives by sulfonated resin and hydrotalcite-supported ruthenium catalysts, ACS Catalysis, 1 (2011) 1562-1565. [149] J. Chen, Y. Guo, J. Chen, L. Song, L. Chen, One-Step Approach to 2, 5- 
Diformylfuran from Fructose by Proton-and Vanadium-Containing Graphitic Carbon Nitride, ChemCatChem, 6 (2014) 3174-3181.

[150] C.L. Hill, Introduction: polyoxometalates multicomponent molecular vehicles to probe fundamental issues and practical problems, Chemical Reviews, $98(1998) 1-2$.

[151] F. Cavani, Heteropolycompound-based catalysts:: A blend of acid and oxidizing properties, Catalysis Today, 41 (1998) 73-86.

[152] W. Deng, Q. Zhang, Y. Wang, Polyoxometalates as efficient catalysts for transformations of cellulose into platform chemicals, Dalton Transactions, 41 (2012) 9817-9831.

[153] Y. Liu, L. Zhu, J. Tang, M. Liu, R. Cheng, C. Hu, One-pot, One-step Synthesis of 2,5-Diformylfuran from Carbohydrates over Mo-Containing Keggin Heteropolyacids, ChemSusChem, 7 (2014) 3541-3547.

[154] R. Liu, J. Chen, L. Chen, Y. Guo, J. Zhong, One-Step Approach to 2, 5Diformylfuran from Fructose by Using a Bifunctional and Recyclable Acidic Polyoxometalate Catalyst, ChemPlusChem, 79 (2014) 1448-1454.

[155] Z. Zhang, B. Liu, K. Lv, J. Sun, K. Deng, Aerobic oxidation of biomass derived 5-hydroxymethylfurfural into 5-hydroxymethyl-2-furancarboxylic acid catalyzed by a montmorillonite K-10 clay immobilized molybdenum acetylacetonate complex, Green Chemistry, 16 (2014) 2762-2770.

[156] N. Mittal, G.M. Nisola, L.B. Malihan, J.G. Seo, H. Kim, S.-P. Lee, W.-J. Chung, One-pot synthesis of 2, 5-diformylfuran from fructose using a magnetic bi-functional catalyst, RSC Advances, 6 (2016) 25678-25688.

[157] G. Lv, H. Wang, Y. Yang, T. Deng, C. Chen, Y. Zhu, X. Hou, Graphene Oxide: A Convenient Metal-Free Carbocatalyst for Facilitating Aerobic Oxidation of 5-Hydroxymethylfurfural into 2, 5-Diformylfuran, ACS Catalysis, 5 (2015) 5636-5646.

[158] F. Rodriguez-Reinoso, The role of carbon materials in heterogeneous 
catalysis, Carbon, 36 (1998) 159-175.

[159] K. Tanabe, Solid acids and bases: their catalytic properties, Elsevier2012. [160] J. Jae, G.A. Tompsett, A.J. Foster, K.D. Hammond, S.M. Auerbach, R.F. Lobo, G.W. Huber, Investigation into the shape selectivity of zeolite catalysts for biomass conversion, Journal of Catalysis, 279 (2011) 257-268.

[161] R. Dimitrijevic, W. Lutz, A. Ritzmann, Hydrothermal stability of zeolites: Determination of extra-framework species of HY faujasite-type steamed zeolite, Journal of Physics and Chemistry of Solids, 67 (2006) 1741-1748.

[162] A.G. Gayubo, A.T. Aguayo, A. Atutxa, R. Aguado, M. Olazar, J. Bilbao, Transformation of oxygenate components of biomass pyrolysis oil on a HZSM5 zeolite. II. Aldehydes, ketones, and acids, Industrial \& Engineering Chemistry Research, 43 (2004) 2619-2626.

[163] N. Salman, C. Rüscher, J.-C. Buhl, W. Lutz, H. Toufar, M. Stöcker, Effect of temperature and time in the hydrothermal treatment of HY zeolite, Microporous and Mesoporous materials, 90 (2006) 339-346.

[164] S. Yan, S.O. Salley, K.S. Ng, Simultaneous transesterification and esterification of unrefined or waste oils over $\mathrm{ZnO}$-La 2 O 3 catalysts, Applied Catalysis A: General, 353 (2009) 203-212.

[165] C. Tagusagawa, A. Takagaki, A. Iguchi, K. Takanabe, J.N. Kondo, K. Ebitani, S. Hayashi, T. Tatsumi, K. Domen, Highly Active Mesoporous Nb-W Oxide Solid-Acid Catalyst, Angewandte Chemie, 122 (2010) 1146-1150.

[166] M. Chidambaram, D. Curulla-Ferre, A. Singh, B. Anderson, Synthesis and characterization of triflic acid-functionalized mesoporous Zr-TMS catalysts: heterogenization of CF 3 SO 3 H over Zr-TMS and its catalytic activity, Journal of Catalysis, 220 (2003) 442-456.

[167] J.N. Kondo, T. Yamashita, K. Nakajima, D. Lu, M. Hara, K. Domen, Preparation and crystallization characteristics of mesoporous TiO 2 and mixed oxides, Journal of Materials Chemistry, 15 (2005) 2035-2040. 
[168] C. Tagusagawa, A. Takagaki, A. Iguchi, K. Takanabe, J.N. Kondo, K. Ebitani, T. Tatsumi, K. Domen, Synthesis and characterization of mesoporous $\mathrm{Ta}-\mathrm{W}$ oxides as strong solid acid catalysts, Chemistry of Materials, 22 (2010) 3072-3078.

[169] S. Ardizzone, C. Bianchi, G. Cappelletti, F. Porta, Liquid-phase catalytic activity of sulfated zirconia from sol-gel precursors: the role of the surface features, Journal of Catalysis, 227 (2004) 470-478.

[170] V. Strelko, Mechanism of the effect of thermal treatment on the formation of strong acid sites in the surface layers of sulfated metal oxides, Kinetics and catalysis, 44 (2003) 834-839.

[171] S. Furuta, H. Matsuhashi, K. Arata, Biodiesel fuel production with solid superacid catalysis in fixed bed reactor under atmospheric pressure, Catalysis communications, 5 (2004) 721-723.

[172] Y. Sun, S. Ma, Y. Du, L. Yuan, S. Wang, J. Yang, F. Deng, F.-S. Xiao, Solvent-free preparation of nanosized sulfated zirconia with Brønsted acidic sites from a simple calcination, The Journal of Physical Chemistry B, 109 (2005) $2567-2572$.

[173] T. Gupta, J. Bechtold, R. Kuznicki, L. Cadoff, B. Rossing, Stabilization of tetragonal phase in polycrystalline zirconia, Journal of Materials Science, 12 (1977) 2421-2426.

[174] M. Sanz, R. Murga, S. Beltran, J.L. Cabezas, J. Coca, Autocatalyzed and ion-exchange-resin-catalyzed esterification kinetics of lactic acid with methanol, Industrial \& engineering chemistry research, 41 (2002) 512-517.

[175] Y. Uozumi, K. Shibatomi, Catalytic asymmetric allylic alkylation in water with a recyclable amphiphilic resin-supported $\mathrm{P}, \mathrm{N}$-chelating palladium complex, Journal of the American Chemical Society, 123 (2001) 2919-2920.

[176] T. Okada, M. Harada, Hydration of halide anions in ion-exchange resin and their dissociation from cationic groups, Analytical chemistry, 76 (2004) 4564- 
4571.

[177] X. Qi, M. Watanabe, T.M. Aida, R.L. Smith Jr, Catalytic dehydration of fructose into 5-hydroxymethylfurfural by ion-exchange resin in mixed-aqueous system by microwave heating, Green Chemistry, 10 (2008) 799-805.

[178] Z.H. Lin, C.J. Guan, X.L. Feng, C.X. Zhao, Synthesis of macroreticular p( $\omega$-sulfonic-perfluoroalkylated) polystyrene ion-exchange resin and its application as solid acid catalyst, Journal of Molecular Catalysis A: Chemical, 247 (2006) 19-26.

[179] N. Hartler, K. Hyllengren, Heterogeneous hydrolysis of cellulose with high polymer acids. Part 3. The acid hydrolysis of cellulose with finely divided cationexchange resin in the hydrogen form, Journal of Polymer Science, 56 (1962) 425434.

[180] S.-J. Kim, A.A. Dwiatmoko, J.W. Choi, Y.-W. Suh, D.J. Suh, M. Oh, Cellulose pretreatment with 1-n-butyl-3-methylimidazolium chloride for solid acid-catalyzed hydrolysis, Bioresource technology, 101 (2010) 8273-8279.

[181] Z. Zhang, Z.K. Zhao, Solid acid and microwave-assisted hydrolysis of cellulose in ionic liquid, Carbohydrate Research, 344 (2009) 2069-2072.

[182] N.-O. Nilvebrant, A. Reimann, S. Larsson, L.J. Jönsson, Detoxification of lignocellulose hydrolysates with ion-exchange resins, Applied biochemistry and biotechnology, 91 (2001) 35-49.

[183] J. Tian, C. Fang, M. Cheng, X. Wang, Hydrolysis of cellulose over CsxH3xPW12O40 $(\mathrm{X}=1-3)$ heteropoly acid catalysts, Chemical Engineering \& Technology, 34 (2011) 482-486.

[184] Y. Zou, B. Yue, B. Zhang, H. He, Solid synthesis of CsxH3-xPW12O40 salts and their catalytic activity for the isomerization of n-butane, Chemistry Letters, 35 (2006) 202-203.

[185] G.M. Maksimov, Advances in the synthesis of polyoxometalates and in the study of heteropolyacids, Russian Chemical Reviews, 64 (1995) 445-461. 
[186] A. Zięba, A. Drelinkiewicz, P. Chmielarz, L. Matachowski, J. Stejskal, Transesterification of triacetin with methanol on various solid acid catalysts: A role of catalyst properties, Applied Catalysis A: General, 387 (2010) 13-25.

[187] C. Rocchiccioli-Deltcheff, M. Amirouche, G. Hervé, M. Fournier, M. Che, J.-M. Tatibouët, Structure and catalytic properties of silica-supported polyoxomolybdates: II. Thermal behavior of unsupported and silica-supported 12-molybdosilicic acid catalysts from IR and catalytic reactivity studies, Journal of Catalysis, 126 (1990) 591-599.

[188] C. Caetano, I. Fonseca, A. Ramos, J. Vital, J. Castanheiro, Esterification of free fatty acids with methanol using heteropolyacids immobilized on silica, Catalysis Communications, 9 (2008) 1996-1999.

[189] L. Pizzio, C. Caceres, M. Blanco, Acid catalysts prepared by impregnation of tungstophosphoric acid solutions on different supports, Applied Catalysis A: General, 167 (1998) 283-294.

[190] G. Sunita, B.M. Devassy, A. Vinu, D.P. Sawant, V. Balasubramanian, S. Halligudi, Synthesis of biodiesel over zirconia-supported isopoly and heteropoly tungstate catalysts, Catalysis Communications, 9 (2008) 696-702.

[191] P. Dupont, F. Lefebvre, Esterification of propanoic acid by butanol and 2ethylhexanol catalyzed by heteropolyacids pure or supported on carbon, Journal of Molecular Catalysis A: Chemical, 114 (1996) 299-307.

[192] T. Blasco, A. Corma, A. Martinez, P. Martinez-Escolano, Supported heteropolyacid (HPW) catalysts for the continuous alkylation of isobutane with 2-butene: The benefit of using MCM-41 with larger pore diameters, Journal of catalysis, 177 (1998) 306-313.

[193] L. Xu, Y. Wang, X. Yang, X. Yu, Y. Guo, J.H. Clark, Preparation of mesoporous polyoxometalate-tantalum pentoxide composite catalyst and its application for biodiesel production by esterification and transesterification, Green Chemistry, 10 (2008) 746-755. 
[194] J.A. Bootsma, B.H. Shanks, Cellobiose hydrolysis using organic-inorganic hybrid mesoporous silica catalysts, Applied Catalysis A: General, 327 (2007) 4451.

[195] A. Takagaki, C. Tagusagawa, K. Domen, Glucose production from saccharides using layered transition metal oxide and exfoliated nanosheets as a water-tolerant solid acid catalyst, Chemical Communications, (2008) 5363-5365. [196] R. Rinaldi, R. Palkovits, F. Schüth, Depolymerization of cellulose using solid catalysts in ionic liquids, Angewandte Chemie International Edition, 47 (2008) 8047-8050.

[197] Z. Yang, L. Niu, Z. Ma, H. Ma, Z. Lei, Fabrication of highly active Sn/W mixed transition-metal oxides as solid acid catalysts, Transition Metal Chemistry, 36 (2011) 269-274.

[198] P. Delaney, C. McManamon, J.P. Hanrahan, M.P. Copley, J.D. Holmes, M.A. Morris, Development of chemically engineered porous metal oxides for phosphate removal, Journal of hazardous materials, 185 (2011) 382-391.

[199] A. Fukuoka, P.L. Dhepe, Catalytic conversion of cellulose into sugar alcohols, Angewandte Chemie International Edition, 45 (2006) 5161-5163.

[200] A. Onda, T. Ochi, K. Yanagisawa, Selective hydrolysis of cellulose into glucose over solid acid catalysts, Green Chemistry, 10 (2008) 1033-1037.

[201] J.T. Yu, A.M. Dehkhoda, N. Ellis, Development of biochar-based catalyst for transesterification of canola oil, Energy \& Fuels, 25 (2010) 337-344.

[202] X. Mo, E. Lotero, C. Lu, Y. Liu, J.G. Goodwin, A novel sulfonated carbon composite solid acid catalyst for biodiesel synthesis, Catalysis Letters, 123 (2008) $1-6$.

[203] L.J. Konwar, J. Boro, D. Deka, Review on latest developments in biodiesel production using carbon-based catalysts, Renewable and Sustainable Energy Reviews, 29 (2014) 546-564.

[204] M. Hara, T. Yoshida, A. Takagaki, T. Takata, J.N. Kondo, S. Hayashi, K. 
Domen, A Carbon Material as a Strong Protonic Acid, Angewandte Chemie International Edition, 43 (2004) 2955-2958.

[205] D. Carpenter, J. Mittendorf, Sulfonated aromatic product and method of manufacturing the same, Google Patents, 1974.

[206] X. Wang, R. Liu, M.M. Waje, Z. Chen, Y. Yan, K.N. Bozhilov, P. Feng, Sulfonated ordered mesoporous carbon as a stable and highly active protonic acid catalyst, Chemistry of Materials, 19 (2007) 2395-2397.

[207] R. Liu, X. Wang, X. Zhao, P. Feng, Sulfonated ordered mesoporous carbon for catalytic preparation of biodiesel, Carbon, 46 (2008) 1664-1669.

[208] Q. Li, S. Chen, L. Zhuang, X. Xu, H. Li, Preparation of a sulfonated activated carbon fiber catalyst with $\gamma$-irradiation-induced grafting method, Journal of Materials Research, 27 (2012) 3083-3089.

[209] D. Zareyee, M.S. Ghandali, M.A. Khalilzadeh, Sulfonated ordered nanoporous carbon (CMK-5-SO3H) as an efficient and highly recyclable catalyst for the silylation of alcohols and phenols with hexamethyldisilazane (HMDS), Catalysis letters, 141 (2011) 1521-1525.

[210] X.-H. Zhang, Q.-Q. Tang, D. Yang, W.-M. Hua, Y.-H. Yue, B.-D. Wang, X.H. Zhang, J.-H. Hu, Preparation of poly (p-styrenesulfonic acid) grafted multiwalled carbon nanotubes and their application as a solid-acid catalyst, Materials Chemistry and Physics, 126 (2011) 310-313.

[211] A. Aldana-Pérez, L. Lartundo-Rojas, R. Gómez, M. Niño-Gómez, Sulfonic groups anchored on mesoporous carbon Starbons-300 and its use for the esterification of oleic acid, Fuel, 100 (2012) 128-138.

[212] G.-J. Sohn, H.-J. Choi, I.-Y. Jeon, D.W. Chang, L. Dai, J.-B. Baek, Waterdispersible, sulfonated hyperbranched poly (ether-ketone) grafted multiwalled carbon nanotubes as oxygen reduction catalysts, ACS nano, 6 (2012) 6345-6355. [213] H. Xiao, Y. Guo, X. Liang, C. Qi, One-step synthesis of a novel carbonbased strong acid catalyst through hydrothermal carbonization, Monatshefte für 
Chemie-Chemical Monthly, 141 (2010) 929-932.

[214] W. Zhang, H. Tao, B. Zhang, J. Ren, G. Lu, Y. Wang, One-pot synthesis of carbonaceous monolith with surface sulfonic groups and its carbonization/activation, Carbon, 49 (2011) 1811-1820.

[215] H. Xiao, Y. Guo, X. Liang, C. Qi, One-step synthesis of novel biacidic carbon via hydrothermal carbonization, Journal of Solid State Chemistry, 183 (2010) 1721-1725.

[216] M. Okamura, A. Takagaki, M. Toda, J.N. Kondo, K. Domen, T. Tatsumi, M. Hara, S. Hayashi, Acid-Catalyzed Reactions on Flexible Polycyclic Aromatic Carbon in Amorphous Carbon, Chemistry of Materials, 18 (2006) 3039-3045. [217] M.-H. Zong, Z.-Q. Duan, W.-Y. Lou, T.J. Smith, H. Wu, Preparation of a sugar catalyst and its use for highly efficient production of biodiesel, Green Chemistry, 9 (2007) 434-437.

[218] S. Suganuma, K. Nakajima, M. Kitano, H. Kato, A. Tamura, H. Kondo, S. Yanagawa, S. Hayashi, M. Hara, SO 3 H-bearing mesoporous carbon with highly selective catalysis, Microporous and Mesoporous Materials, 143 (2011) 443-450. [219] S. Suganuma, K. Nakajima, M. Kitano, D. Yamaguchi, H. Kato, S. Hayashi, M. Hara, Synthesis and acid catalysis of cellulose-derived carbon-based solid acid, Solid State Sciences, 12 (2010) 1029-1034.

[220] X. Tian, L.L. Zhang, P. Bai, X. Zhao, Sulfonic-acid-functionalized porous benzene phenol polymer and carbon for catalytic esterification of methanol with acetic acid, Catalysis today, 166 (2011) 53-59.

[221] M.A. Harmer, Q. Sun, Solid acid catalysis using ion-exchange resins, Applied Catalysis A: General, 221 (2001) 45-62.

[222] S. Suganuma, K. Nakajima, M. Kitano, S. Hayashi, M. Hara, sp3-Linked Amorphous Carbon with Sulfonic Acid Groups as a Heterogeneous Acid Catalyst, ChemSusChem, 5 (2012) 1841-1846.

[223] K. Nakajima, M. Hara, Amorphous carbon with SO3H groups as a solid 
Brønsted acid catalyst, ACS catalysis, 2 (2012) 1296-1304.

[224] S. Dora, T. Bhaskar, R. Singh, D.V. Naik, D.K. Adhikari, Effective catalytic conversion of cellulose into high yields of methyl glucosides over sulfonated carbon based catalyst, Bioresource Technology, 120 (2012) 318-321.

[225] A. Onda, T. Ochi, K. Yanagisawa, Hydrolysis of cellulose selectively into glucose over sulfonated activated-carbon catalyst under hydrothermal conditions, Topics in Catalysis, 52 (2009) 801-807.

[226] C. Zhang, H. Wang, F. Liu, L. Wang, H. He, Magnetic core-shell Fe3O4@ C-SO3H nanoparticle catalyst for hydrolysis of cellulose, Cellulose, 20 (2013) 127-134.

[227] H. Guo, X. Qi, L. Li, R.L. Smith, Hydrolysis of cellulose over functionalized glucose-derived carbon catalyst in ionic liquid, Bioresource technology, 116 (2012) 355-359.

[228] D. Yamaguchi, M. Kitano, S. Suganuma, K. Nakajima, H. Kato, M. Hara, Hydrolysis of cellulose by a solid acid catalyst under optimal reaction conditions, The Journal of Physical Chemistry C, 113 (2009) 3181-3188.

[229] J. Wang, W. Xu, J. Ren, X. Liu, G. Lu, Y. Wang, Efficient catalytic conversion of fructose into hydroxymethylfurfural by a novel carbon-based solid acid, Green Chemistry, 13 (2011) 2678-2681.

[230] E. Lam, J.H. Chong, E. Majid, Y. Liu, S. Hrapovic, A.C. Leung, J.H. Luong, Carbocatalytic dehydration of xylose to furfural in water, Carbon, 50 (2012) 1033-1043.

[231] R. Ormsby, J.R. Kastner, J. Miller, Hemicellulose hydrolysis using solid acid catalysts generated from biochar, Catalysis today, 190 (2012) 89-97.

[232] X. Qi, H. Guo, L. Li, R.L. Smith, Acid-Catalyzed Dehydration of Fructose into 5-Hydroxymethylfurfural by Cellulose-Derived Amorphous Carbon, ChemSusChem, 5 (2012) 2215-2220.

[233] A.M. Dehkhoda, A.H. West, N. Ellis, Biochar based solid acid catalyst for 
biodiesel production, Applied Catalysis A: General, 382 (2010) 197-204.

[234] W. Zhao, B. Yang, C. Yi, Z. Lei, J. Xu, Etherification of glycerol with isobutylene to produce oxygenate additive using sulfonated peanut shell catalyst, Industrial \& Engineering Chemistry Research, 49 (2010) 12399-12404.

[235] Q. Shu, Q. Zhang, G. Xu, Z. Nawaz, D. Wang, J. Wang, Synthesis of biodiesel from cottonseed oil and methanol using a carbon-based solid acid catalyst, Fuel Processing Technology, 90 (2009) 1002-1008.

[236] Q. Shu, Q. Zhang, G. Xu, J. Wang, Preparation of biodiesel using sMWCNT catalysts and the coupling of reaction and separation, food and bioproducts processing, 87 (2009) 164-170.

[237] W.-Y. Lou, M.-H. Zong, Z.-Q. Duan, Efficient production of biodiesel from high free fatty acid-containing waste oils using various carbohydrate-derived solid acid catalysts, Bioresource Technology, 99 (2008) 8752-8758.

[238] B. Rao, K.C. Mouli, N. Rambabu, A. Dalai, R. Prasad, Carbon-based solid acid catalyst from de-oiled canola meal for biodiesel production, Catalysis Communications, 14 (2011) 20-26.

[239] J. Ma, S. Ng, Y. Yong, X.-Z. Luo, X. Wang, X.-W. Liu, Recyclable Sulfonated Amorphous Carbon Catalyzed Friedel-Crafts Alkylation of Indoles with $\alpha, \beta$-Unsaturated Carbonyl Compounds in Water, Chemistry - An Asian Journal, 5 (2010) 778-782.

[240] K. Liu, C. Li, X. Zhang, W. Hua, D. Yang, J. Hu, Y. Yue, Z. Gao, Poly (styrene sulfonic acid)-grafted carbon nanotube as a stable protonic acid catalyst, Catalysis Communications, 12 (2010) 217-221.

[241] X. Liang, M. Zeng, C. Qi, One-step synthesis of carbon functionalized with sulfonic acid groups using hydrothermal carbonization, Carbon, 48 (2010) 18441848.

[242] X. Liang, H. Xiao, Y. Shen, C. Qi, One-step synthesis of novel sulfuric acid groups' functionalized carbon via hydrothermal carbonization, Materials Letters, 
$64(2010)$ 953-955.

[243] K. Nakajima, M. Okamura, J.N. Kondo, K. Domen, T. Tatsumi, S. Hayashi, M. Hara, Amorphous carbon bearing sulfonic acid groups in mesoporous silica as a selective catalyst, Chemistry of Materials, 21 (2008) 186-193.

[244] T. Ramulifho, K.I. Ozoemena, R.M. Modibedi, C.J. Jafta, M.K. Mathe, Electrocatalytic oxidation of ethylene glycol at palladium-bimetallic nanocatalysts (PdSn and PdNi) supported on sulfonate-functionalised multiwalled carbon nanotubes, Journal of Electroanalytical Chemistry, 692 (2013) 2630.

[245] T. Ramulifho, K.I. Ozoemena, R.M. Modibedi, C.J. Jafta, M.K. Mathe, Fast microwave-assisted solvothermal synthesis of metal nanoparticles ( $\mathrm{Pd}, \mathrm{Ni}, \mathrm{Sn})$ supported on sulfonated MWCNTs: Pd-based bimetallic catalysts for ethanol oxidation in alkaline medium, Electrochimica Acta, 59 (2012) 310-320.

[246] B.P. Devi, K. Gangadhar, K.S. Kumar, K.S. Shanker, R. Prasad, P.S. Prasad, Synthesis of sulfonic acid functionalized carbon catalyst from glycerol pitch and its application for tetrahydropyranyl protection/deprotection of alcohols and phenols, Journal of Molecular Catalysis A: Chemical, 345 (2011) 96-100.

[247] E.H. Falcao, F. Wudl, Carbon allotropes: beyond graphite and diamond, Journal of Chemical Technology and Biotechnology, 82 (2007) 524-531.

[248] M.-M. Titirici, M. Antonietti, Chemistry and materials options of sustainable carbon materials made by hydrothermal carbonization, Chemical Society Reviews, 39 (2010) 103-116.

[249] P. Ajayan, Nanotubes from carbon, Chemical reviews, 99 (1999) 1787.

[250] F. Schedin, A. Geim, S. Morozov, E. Hill, P. Blake, M. Katsnelson, K. Novoselov, Detection of individual gas molecules adsorbed on graphene, Nature materials, 6 (2007) 652-655.

[251] K. Zhang, Q. Yue, G. Chen, Y. Zhai, L. Wang, H. Wang, J. Zhao, J. Liu, J. Jia, H. Li, Effects of acid treatment of Pt-Ni alloy nanoparticles@, graphene on 
the kinetics of the oxygen reduction reaction in acidic and alkaline solutions, The Journal of Physical Chemistry C, 115 (2010) 379-389.

[252] K.-W. Nam, J. Song, K.-H. Oh, M.-J. Choo, H. Park, J.-K. Park, J.W. Choi, Monodispersed PtCo nanoparticles on hexadecyltrimethylammonium bromide treated graphene as an effective oxygen reduction reaction catalyst for proton exchange membrane fuel cells, Carbon, 50 (2012) 3739-3747.

[253] B. Vinayan, S. Ramaprabhu, Platinum-TM (TM= Fe, Co) alloy nanoparticles dispersed nitrogen doped (reduced graphene oxide-multiwalled carbon nanotube) hybrid structure cathode electrocatalysts for high performance PEMFC applications, Nanoscale, 5 (2013) 5109-5118.

[254] Y. Shao, S. Zhang, C. Wang, Z. Nie, J. Liu, Y. Wang, Y. Lin, Highly durable graphene nanoplatelets supported Pt nanocatalysts for oxygen reduction, Journal of Power Sources, 195 (2010) 4600-4605.

[255] X.-K. Kong, C.-L. Chen, Q.-W. Chen, Doped graphene for metal-free catalysis, Chemical Society Reviews, 43 (2014) 2841-2857.

[256] R.I. Jafri, N. Rajalakshmi, S. Ramaprabhu, Nitrogen doped graphene nanoplatelets as catalyst support for oxygen reduction reaction in proton exchange membrane fuel cell, Journal of Materials Chemistry, 20 (2010) 71147117.

[257] V. Budarin, J.H. Clark, J.J. Hardy, R. Luque, K. Milkowski, S.J. Tavener, A.J. Wilson, Starbons: New Starch-Derived Mesoporous Carbonaceous Materials with Tunable Properties, Angewandte Chemie, 118 (2006) 3866-3870. [258] P.S. Shuttleworth, V. Budarin, R.J. White, V.M. Gun'ko, R. Luque, J.H. Clark, Molecular-Level Understanding of the Carbonisation of Polysaccharides, Chemistry-A European Journal, 19 (2013) 9351-9357.

[259] R.J. White, V.L. Budarin, J.H. Clark, Tuneable Mesoporous Materials from a-D-Polysaccharides, ChemSusChem, 1 (2008) 408-411.

[260] J. Brunelle, Preparation of catalysts by metallic complex adsorption on 
mineral oxides, Pure and Applied Chemistry, 50 (1978) 1211-1229.

[261] C. Prado-Burguete, A. Linares-Solano, F. Rodriguez-Reinoso, C.S.-M. De Lecea, The effect of oxygen surface groups of the support on platinum dispersion in Pt/carbon catalysts, Journal of Catalysis, 115 (1989) 98-106.

[262] D.J. Suh, P. Tae-Jin, I. Son-Ki, Effect of surface oxygen groups of carbon supports on the characteristics of Pd/C catalysts, Carbon, 31 (1993) 427-435.

[263] H. Zhu, W. Han, H. Liu, Influence of oxidation on heat-treated activated carbon support properties and metallic dispersion of $\mathrm{Ru} / \mathrm{C}$ catalyst, Catalysis letters, 115 (2007) 13-18.

[264] W. Han, H. Liu, H. Zhu, Effect of activated carbon on the dispersion of Ru and $\mathrm{K}$ over supported Ru-based catalyst for ammonia synthesis, Catalysis Communications, 8 (2007) 351-354.

[265] M.L. Toebes, M.K. van der Lee, L.M. Tang, M.H. Huis in 't Veld, J.H. Bitter, A.J. van Dillen, K.P. de Jong, Preparation of Carbon Nanofiber Supported Platinum and Ruthenium Catalysts: Comparison of Ion Adsorption and Homogeneous Deposition Precipitation, The Journal of Physical Chemistry B, 108 (2004) 11611-11619.

[266] M. Gurrath, T. Kuretzky, H. Boehm, L. Okhlopkova, A. Lisitsyn, V. Likholobov, Palladium catalysts on activated carbon supports: Influence of reduction temperature, origin of the support and pretreatments of the carbon surface, Carbon, 38 (2000) 1241-1255.

[267] H. Van Dam, H. Van Bekkum, Preparation of platinum on activated carbon, Journal of Catalysis, 131 (1991) 335-349.

[268] A. Sepúlveda-Escribano, F. Coloma, F. Rodriguez-Reinoso, Platinum catalysts supported on carbon blacks with different surface chemical properties, Applied Catalysis A: General, 173 (1998) 247-257.

[269] M. Fraga, E. Jordao, M. Mendes, M. Freitas, J. Faria, J. Figueiredo, Properties of carbon-supported platinum catalysts: role of carbon surface sites, 
Journal of Catalysis, 209 (2002) 355-364.

[270] J.H. Bitter, K.P. De Jong, Preparation of carbon-supported metal catalysts, Carbon Materials for Catalysis, John Wiley \& Sons2009, pp. 157-176.

[271] J. Bitter, M. Van der Lee, A. Slotboom, A. Van Dillen, K. De Jong, Synthesis of highly loaded highly dispersed nickel on carbon nanofibers by homogeneous deposition-precipitation, Catalysis letters, 89 (2003) 139-142.

[272] A. Fuente, G. Pulgar, F. González, C. Pesquera, C. Blanco, Activated carbon supported Pt catalysts: effect of support texture and metal precursor on activity of acetone hydrogenation, Applied Catalysis A: General, 208 (2001) 3546.

[273] P. Samant, C. Rangel, M. Romero, J. Fernandes, J. Figueiredo, Carbon supports for methanol oxidation catalyst, Journal of power sources, 151 (2005) $79-84$.

[274] P. Burattin, M. Che, C. Louis, Characterization of the Ni (II) phase formed on silica upon deposition-precipitation, The Journal of Physical Chemistry B, 101 (1997) 7060-7074.

[275] P. Burattin, M. Che, C. Louis, Metal particle size in $\mathrm{Ni} / \mathrm{SiO} 2$ materials prepared by deposition-precipitation: influence of the nature of the Ni (II) phase and of its interaction with the support, The Journal of Physical Chemistry B, 103 (1999) 6171-6178.

[276] M.K. van der Lee, J. van Dillen, J.H. Bitter, K.P. de Jong, Deposition precipitation for the preparation of carbon nanofiber supported nickel catalysts, Journal of the American Chemical Society, 127 (2005) 13573-13582.

[277] R. Bachir, P. Marecot, B. Didillon, J. Barbier, Isoprene hydrogenation on supported $\mathrm{Pd} \mathrm{Fe}$ catalysts. Influence of the catalyst preparation procedure, Applied Catalysis A: General, 164 (1997) 313-322.

[278] G. Corro, P. Marecot, J. Barbier, Deactivation of Pt-Sn/A1203 Catalysts by Coking: Influence of the Preparation Method, Catalyst Deactivation 1997, 111 
(1997) 359.

[279] G. Heal, L. Mkayula, The preparation of palladium metal catalysts supported on carbon part II: Deposition of palladium and metal area measurements, Carbon, 26 (1988) 815-823.

[280] Y. Yang, Y. Zhou, C. Cha, W.M. Carroll, A new method for the preparation of highly dispersed metal/carbon catalyst- $\mathrm{Pd} / \mathrm{C}$ catalyst and its properties, Electrochimica acta, 38 (1993) 2333-2341.

[281] L. Ang, T.A. Hor, G. Xu, C. Tung, S. Zhao, J.L. Wang, Decoration of activated carbon nanotubes with copper and nickel, Carbon, 38 (2000) 363-372. [282] P. Shen, N. Chi, K.-Y. Chan, D. Phillips, Platinum nanoparticles spontaneously formed on HOPG, Applied surface science, 172 (2001) 159-166. [283] H.C. Choi, M. Shim, S. Bangsaruntip, H. Dai, Spontaneous reduction of metal ions on the sidewalls of carbon nanotubes, Journal of the American Chemical Society, 124 (2002) 9058-9059.

[284] C.M. Lok, Novel highly dispersed cobalt catalysts for improved FischerTropsch productivity, Studies in surface science and catalysis, 147 (2004) 283288.

[285] L. Hermans, J. Geus, Interaction of nickel ions with silica supports during deposition-precipitation, Studies in Surface Science and Catalysis, 3 (1979) 113130.

[286] G.L. Bezemer, P. Radstake, V. Koot, A. Van Dillen, J. Geus, K. De Jong, Preparation of Fischer-Tropsch cobalt catalysts supported on carbon nanofibers and silica using homogeneous deposition-precipitation, Journal of catalysis, 237 (2006) 291-302.

[287] W. Li, C. Liang, W. Zhou, J. Qiu, Z. Zhou, G. Sun, Q. Xin, Preparation and characterization of multiwalled carbon nanotube-supported platinum for cathode catalysts of direct methanol fuel cells, The Journal of Physical Chemistry B, 107 (2003) 6292-6299. 
[288] W.X. Chen, J.Y. Lee, Z. Liu, Microwave-assisted synthesis of carbon supported Pt nanoparticles for fuel cell applications, Chemical Communications, (2002) 2588-2589.

[289] B. Yang, Q. Lu, Y. Wang, L. Zhuang, J. Lu, P. Liu, J. Wang, R. Wang, Simple and low-cost preparation method for highly dispersed $\mathrm{PtRu} / \mathrm{C}$ catalysts, Chemistry of Materials, 15 (2003) 3552-3557.

[290] T. Komanoya, H. Kobayashi, K. Hara, W.J. Chun, A. Fukuoka, Kinetic Study of Catalytic Conversion of Cellulose to Sugar Alcohols under LowPressure Hydrogen, ChemCatChem, 6 (2014) 230-236.

[291] Y. Zhang, A. Wang, T. Zhang, A new 3D mesoporous carbon replicated from commercial silica as a catalyst support for direct conversion of cellulose into ethylene glycol, Chemical Communications, 46 (2010) 862-864.

[292] P.A. Russo, S. Lima, V. Rebuttini, M. Pillinger, M.-G. Willinger, N. Pinna, A.A. Valente, Microwave-assisted coating of carbon nanostructures with titanium dioxide for the catalytic dehydration of d-xylose into furfural, RSC Advances, 3 (2013) 2595-2603.

[293] D. Wang, W. Niu, M. Tan, M. Wu, X. Zheng, Y. Li, N. Tsubaki, Pt nanocatalysts supported on reduced graphene oxide for selective conversion of cellulose or cellobiose to sorbitol, ChemSusChem, 7 (2014) 1398-1406.

[294] M. Härkönen, P. Nuojua, Eri tekijöiden vaikutus ksyloosin katalyyyttiseen hydraukseen ksylitoliksi, Kemia-Kemi, 6 (1979) 445-447.

[295] X. Liang, C. Jiang, Atomic layer deposited highly dispersed platinum nanoparticles supported on non-functionalized multiwalled carbon nanotubes for the hydrogenation of xylose to xylitol, Journal of nanoparticle research, 15 (2013) $1-9$.

[296] H. Ren, Y. Chen, Y. Huang, W. Deng, D.G. Vlachos, J.G. Chen, Tungsten carbides as selective deoxygenation catalysts: experimental and computational studies of converting C3 oxygenates to propene, Green Chemistry, 16 (2014) 
761-769.

[297] H. Ren, W. Yu, M. Salciccioli, Y. Chen, Y. Huang, K. Xiong, D.G. Vlachos,

J.G. Chen, Selective Hydrodeoxygenation of Biomass-Derived Oxygenates to Unsaturated Hydrocarbons using Molybdenum Carbide Catalysts, ChemSusChem, 6 (2013) 798-801.

[298] J. Han, J. Duan, P. Chen, H. Lou, X. Zheng, Molybdenum CarbideCatalyzed Conversion of Renewable Oils into Diesel-like Hydrocarbons, Advanced Synthesis \& Catalysis, 353 (2011) 2577-2583.

[299] J. Han, J. Duan, P. Chen, H. Lou, X. Zheng, H. Hong, Nanostructured molybdenum carbides supported on carbon nanotubes as efficient catalysts for one-step hydrodeoxygenation and isomerization of vegetable oils, Green Chemistry, 13 (2011) 2561-2568.

[300] R.W. Gosselink, D.R. Stellwagen, J.H. Bitter, Tungsten-Based Catalysts for Selective Deoxygenation, Angewandte Chemie, 125 (2013) 5193-5196.

[301] J. Sun, H. Liu, Selective hydrogenolysis of biomass-derived xylitol to ethylene glycol and propylene glycol on supported Ru catalysts, Green Chemistry, 13 (2011) 135-142.

[302] A.V. Kirilin, B. Hasse, A.V. Tokarev, L.M. Kustov, G.N. Baeva, G.O. Bragina, A.Y. Stakheev, A.-R. Rautio, T. Salmi, B.J. Etzold, Aqueous-phase reforming of xylitol over $\mathrm{Pt} / \mathrm{C}$ and $\mathrm{Pt} / \mathrm{TiC}-\mathrm{CDC}$ catalysts: catalyst characterization and catalytic performance, Catalysis Science \& Technology, 4 (2014) 387-401.

[303] J. Sun, H. Liu, Selective hydrogenolysis of biomass-derived xylitol to ethylene glycol and propylene glycol on $\mathrm{Ni} / \mathrm{C}$ and basic oxide-promoted $\mathrm{Ni} / \mathrm{C}$ catalysts, Catalysis Today, 234 (2014) 75-82.

[304] S.E. Davis, M.S. Ide, R.J. Davis, Selective oxidation of alcohols and aldehydes over supported metal nanoparticles, Green Chemistry, 15 (2013) 1745. 
[305] A. Villa, M. Schiavoni, S. Campisi, G.M. Veith, L. Prati, Pd-modified Au on Carbon as an Effective and Durable Catalyst for the Direct Oxidation of HMF to 2, 5-Furandicarboxylic Acid, ChemSusChem, 6 (2013) 609-612.

[306] X. Wan, C. Zhou, J. Chen, W. Deng, Q. Zhang, Y. Yang, Y. Wang, Basefree aerobic oxidation of 5-hydroxymethyl-furfural to 2, 5-furandicarboxylic acid in water catalyzed by functionalized carbon nanotube-supported $\mathrm{Au}-\mathrm{Pd}$ alloy nanoparticles, ACS Catalysis, 4 (2014) 2175-2185.

[307] M. Zhang, D. He, L. Ji, B. Wei, D. Wu, X. Zhang, Y. Xu, W. Wang, Macroscopic synthesis of onion-like graphitic particles, Nanostructured materials, 10 (1998) 291-297.

[308] T. Cabioc'h, E. Thune, M. Jaouen, Carbon-onion thin-film synthesis onto silica substrates, Chemical Physics Letters, 320 (2000) 202-205.

[309] T. Cabioc'h, E. Thune, M. Jaouen, Mechanisms involved in the formation of onionlike carbon nanostructures synthesized by ion implantation at high temperature, Physical Review B, 65 (2002) 132103.

[310] R.K. Rana, A. Gedanken, Carbon nanoflask: A mechanistic elucidation of its formation, The Journal of Physical Chemistry B, 106 (2002) 9769-9776.

[311] Z. Wang, J. Yin, Graphitic hollow carbon calabashes, Chemical physics letters, 289 (1998) 189-192.

[312] J. Du, Z. Liu, Z. Li, B. Han, Z. Sun, Y. Huang, Carbon onions synthesized via thermal reduction of glycerin with magnesium, Materials chemistry and physics, 93 (2005) 178-180.

[313] T. Stöckli, J.-M. Bonard, A. Châtelain, Z.L. Wang, P. Stadelmann, Plasmon excitations in graphitic carbon spheres, Physical Review B, 57 (1998) 15599.

[314] W.A. de Heer, D. Ugarte, Carbon onions produced by heat treatment of carbon soot and their relation to the $217.5 \mathrm{~nm}$ interstellar absorption feature, Chemical physics letters, 207 (1993) 480-486.

[315] Z. Kang, Z. Wang, Mixed-valent oxide-catalytic carbonization for 
synthesis of monodispersed nano sized carbon spheres, Philosophical Magazine B, 73 (1996) 905-929.

[316] P. Serp, R. Feurer, P. Kalck, Y. Kihn, J. Faria, J. Figueiredo, A chemical vapour deposition process for the production of carbon nanospheres, Carbon, 39 (2001) 621-626.

[317] V.G. Pol, S.V. Pol, J.M. Calderon-Moreno, M.-G. Sung, S. Asai, A. Gedanken, The dependence of the oriented growth of carbon filaments on the intensity of a magnetic field, Carbon, 44 (2006) 1913-1918.

[318] V.G. Pol, S.V. Pol, A. Gedanken, M.-G. Sung, S. Asai, Magnetic field guided formation of long carbon filaments (sausages), Carbon, 42 (2004) 27382741.

[319] X. He, F. Wu, M. Zheng, The synthesis of carbon nanoballs and its electrochemical performance, Diamond and related materials, 16 (2007) 311-315. [320] J. Qiu, Y. Li, Y. Wang, C. Liang, T. Wang, D. Wang, A novel form of carbon micro-balls from coal, Carbon, 41 (2003) 767-772.

[321] Y. Ma, Z. Hu, K. Huo, Y. Lu, Y. Hu, Y. Liu, J. Hu, Y. Chen, A practical route to the production of carbon nanocages, Carbon, 43 (2005) 1667-1672.

[322] G. Yang, Q. Xu, H. Tian, X. Wang, W. Zheng, Amorphous hollow carbon spheres synthesized using radio frequency plasma-enhanced chemical vapour deposition, Journal of Physics D: Applied Physics, 41 (2008) 195504.

[323] H.-s. Qian, F.-m. Han, B. Zhang, Y.-c. Guo, J. Yue, B.-x. Peng, Noncatalytic CVD preparation of carbon spheres with a specific size, Carbon, 42 (2004) 761-766.

[324] M. Aili, W. Xiaomin, L. Tianbao, L. Xuguang, X. Bingshe, Characteristics of carbon microspheres and study on its adsorption isotherms, Materials Science and Engineering: A, 443 (2007) 54-59.

[325] Y.Z. Jin, C. Gao, W.K. Hsu, Y. Zhu, A. Huczko, M. Bystrzejewski, M. Roe, C.Y. Lee, S. Acquah, H. Kroto, Large-scale synthesis and characterization of 
carbon spheres prepared by direct pyrolysis of hydrocarbons, Carbon, 43 (2005) 1944-1953.

[326] X. Wang, J. Guo, X. Yang, B. Xu, Monodisperse carbon microspheres synthesized from asphaltene, Materials Chemistry and Physics, 113 (2009) 821823.

[327] S. Zhao, C. Wang, M. Chen, Z. Shi, Preparation of carbon sphere from corn starch by a simple method, Materials Letters, 62 (2008) 3322-3324.

[328] B. Zhang, S. Bai, H.-M. Cheng, Q.-K. Cai, Graphitization-induced microstructural changes in tetrahydrofuran-derived pyrolytic carbon spheres, Journal of materials research, 21 (2006) 2198-2203.

[329] B. Geng, J. Ma, Q. Du, X. Liu, L. Zhang, Synthesis of hollow carbon nanospheres through a $\mathrm{ZnSe}$ nanoparticle template route, Materials Science and Engineering: A, 466 (2007) 96-100.

[330] W. Li, D. Chen, Z. Li, Y. Shi, Y. Wan, G. Wang, Z. Jiang, D. Zhao, Nitrogencontaining carbon spheres with very large uniform mesopores: the superior electrode materials for EDLC in organic electrolyte, Carbon, 45 (2007) 17571763.

[331] H. Miguez, N. Tetreault, B. Hatton, S.M. Yang, D. Perovic, G.A. Ozin, Mechanical stability enhancement by pore size and connectivity control in colloidal crystals by layer-by-layer growth of oxide, Chemical Communications, (2002) 2736-2737.

[332] J.-Y. Miao, D.W. Hwang, K.V. Narasimhulu, P.-I. Lin, Y.-T. Chen, S.-H. Lin, L.-P. Hwang, Synthesis and properties of carbon nanospheres grown by CVD using Kaolin supported transition metal catalysts, Carbon, 42 (2004) 813822.

[333] J.-Y. Miao, D.W. Hwang, C.-C. Chang, S.-H. Lin, K. Narasimhulu, L.-P. Hwang, Uniform carbon spheres of high purity prepared on kaolin by CCVD, Diamond and related materials, 12 (2003) 1368-1372. 
[334] C. He, N. Zhao, C. Shi, X. Du, J. Li, L. Cui, A practical method for the production of hollow carbon onion particles, Journal of alloys and compounds, 425 (2006) 329-333.

[335] S. Amelinckx, X. Zhang, D. Bernaerts, X. Zhang, V. Ivanov, J. Nagy, A formation mechanism for catalytically grown helix-shaped graphite nanotubes, Opt. Commun, 2664 (1994) 977.

[336] Z. Wang, Z. Kang, Graphitic structure and surface chemical activity of nanosize carbon spheres, Carbon, 35 (1997) 419-426.

[337] V.G. Pol, M. Motiei, A. Gedanken, J. Calderon-Moreno, M. Yoshimura, Carbon spherules: synthesis, properties and mechanistic elucidation, Carbon, 42 (2004) 111-116.

[338] V.G. Pol, S.V. Pol, J.M.C. Moreno, A. Gedanken, High yield one-step synthesis of carbon spheres produced by dissociating individual hydrocarbons at their autogenic pressure at low temperatures, Carbon, 44 (2006) 3285-3292.

[339] S.V. Pol, V.G. Pol, D. Sherman, A. Gedanken, A solvent free process for the generation of strong, conducting carbon spheres by the thermal degradation of waste polyethylene terephthalate, Green Chemistry, 11 (2009) 448-451.

[340] Q. Wang, H. Li, L. Chen, X. Huang, Monodispersed hard carbon spherules with uniform nanopores, Carbon, 39 (2001) 2211-2214.

[341] F.P. Hu, Z. Wang, Y. Li, C. Li, X. Zhang, P.K. Shen, Improved performance of Pd electrocatalyst supported on ultrahigh surface area hollow carbon spheres for direct alcohol fuel cells, Journal of Power Sources, 177 (2008) 61-66.

[342] Y. Xie, Q. Huang, B. Huang, Preparation of high purity carbon nanospheres by the chemical reaction of calcium carbide and oxalic acid, Carbon, 47 (2009) $2292-2295$.

[343] L. Xu, W. Zhang, Q. Yang, Y. Ding, W. Yu, Y. Qian, A novel route to hollow and solid carbon spheres, Carbon, 43 (2005) 1090-1092.

[344] B. Liu, D. Jia, Y. Shao, J. Rao, Effects of gas pressure and temperature on 
the synthesis of hollow carbon spheres in argon atmosphere, Materials Chemistry and Physics, 114 (2009) 391-397.

[345] C. Wu, X. Zhu, L. Ye, C. OuYang, S. Hu, L. Lei, Y. Xie, Necklace-like hollow carbon nanospheres from the pentagon-including reactants: synthesis and electrochemical properties, Inorganic chemistry, 45 (2006) 8543-8550.

[346] G. Hu, D. Ma, M. Cheng, L. Liu, X. Bao, Direct synthesis of uniform hollow carbon spheres by a self-assembly template approach, Chemical Communications, (2002) 1948-1949.

[347] Y. Xiong, Y. Xie, Z. Li, C. Wu, R. Zhang, A novel approach to carbon hollow spheres and vessels from $\mathrm{CCl} 4$ at low temperatures, Chemical Communications, (2003) 904-905.

[348] J. Liu, M. Shao, Q. Tang, X. Chen, Z. Liu, Y. Qian, A medial-reduction route to hollow carbon spheres, Carbon, 41 (2003) 1682-1685.

[349] X. Ma, F. Xu, L. Chen, Y. Zhang, Z. Zhang, J. Qian, Y. Qian, Easy nickel substrate-assisted growth of uniform carbon microspheres and their spectroscopic properties, Carbon, 44 (2006) 2861-2864.

[350] X. Ma, F. Xu, Y. Du, L. Chen, Z. Zhang, Copper substrate-assisted growth of ellipsoidal carbon microparticles, Carbon, 44 (2006) 179-181.

[351] J. Shen, J. Li, Z. Huang, Q. Chen, S. Zhang, Y. Qian, A simple route for the synthesis of coral-like accretion of hollow carbon microspheres with thin walls, Carbon, 44 (2006) 2171-2177.

[352] V.O. Nyamori, N.J. Coville, Effect of ferrocene/carbon ratio on the size and shape of carbon nanotubes and microspheres, Organometallics, 26 (2007) 40834085 .

[353] G. Yu, B. Sun, Y. Pei, S. Xie, S. Yan, M. Qiao, K. Fan, X. Zhang, B. Zong, Fex Oy@C spheres as an excellent catalyst for Fischer- Tropsch synthesis, Journal of the American Chemical Society, 132 (2009) 935-937.

[354] Y. Wang, H. Sun, H.M. Ang, M.O. Tadé, S. Wang, Magnetic Fe 3 O 
4/carbon sphere/cobalt composites for catalytic oxidation of phenol solutions with sulfate radicals, Chemical Engineering Journal, 245 (2014) 1-9.

[355] W. Zhao, Y. Wang, Y. Yang, J. Tang, Y. Yang, Carbon spheres supported visible-light-driven $\mathrm{CuO}-\mathrm{BiVO} 4$ heterojunction: preparation, characterization, and photocatalytic properties, Applied Catalysis B: Environmental, 115 (2012) 90-99.

[356] Z.-X. Liu, J.-N. Park, S. Abdi, S.-K. Park, Y.-K. Park, C.W. Lee, Nanosized carbon hollow spheres for abatement of ethylene, Topics in catalysis, 39 (2006) 221-226.

[357] Y. Liu, X. Qiu, Y. Huang, W. Zhu, G. Wu, Influence of preparation process of MEA with mesocarbon microbeads supported $\mathrm{Pt}-\mathrm{Ru}$ catalysts on methanol electrooxidation, Journal of applied electrochemistry, 32 (2002) 1279-1285.

[358] J.H. Kim, B. Fang, M. Kim, J.-S. Yu, Hollow spherical carbon with mesoporous shell as a superb anode catalyst support in proton exchange membrane fuel cell, Catalysis Today, 146 (2009) 25-30.

[359] C. Tan, F. Wang, J. Liu, Y. Zhao, J. Wang, L. Zhang, K.C. Park, M. Endo, An easy route to prepare carbon black-silver hybrid catalysts for electro-catalytic oxidation of hydrazine, Materials Letters, 63 (2009) 969-971.

[360] B.E. Warren, X-ray Diffraction, Courier Corporation 1969.

[361] Y.Z. Jin, Y.J. Kim, C. Gao, Y.Q. Zhu, A. Huczko, M. Endo, H.W. Kroto, High temperature annealing effects on carbon spheres and their applications as anode materials in Li-ion secondary battery, Carbon, 44 (2006) 724-729.

[362] B. Warren, X-Ray Diffraction Study of Carbon Black, The Journal of Chemical Physics, 2 (1934) 551-555.

[363] V. Drits, J. Srodon, D. Eberl, XRD measurement of mean crystallite thickness of illite and illite/smectite: Reappraisal of the Kubler index and the Scherrer equation, Clays and clay minerals, 45 (1997) 461-475.

[364] E.N. Konyushenko, J. Stejskal, M. Trchová, J. Hradil, J. Kovářová, J. 
Prokeš, M. Cieslar, J.-Y. Hwang, K.-H. Chen, I. Sapurina, Multi-wall carbon nanotubes coated with polyaniline, Polymer, 47 (2006) 5715-5723.

[365] Y.-L. Liu, Y.-H. Chang, M. Liang, Poly (2, 6-dimethyl-1, 4-phenylene oxide)(PPO) multi-bonded carbon nanotube (CNT): Preparation and formation of PPO/CNT nanocomposites, Polymer, 49 (2008) 5405-5409.

[366] H. Zhang, X. Pan, X. Han, X. Liu, X. Wang, W. Shen, X. Bao, Enhancing chemical reactions in a confined hydrophobic environment: An NMR study of benzene hydroxylation in carbon nanotubes, Chemical Science, 4 (2013) 10751078.

[367] G. Giambastiani, S. Cicchi, A. Giannasi, L. Luconi, A. Rossin, F. Mercuri, C. Bianchini, A. Brandi, M. Melucci, G. Ghini, Functionalization of multiwalled carbon nanotubes with cyclic nitrones for materials and composites: addressing the role of CNT sidewall defects, Chemistry of Materials, 23 (2011) 1923-1938. [368] E.P. Barrett, L.G. Joyner, P.P. Halenda, The determination of pore volume and area distributions in porous substances. I. Computations from nitrogen isotherms, Journal of the American Chemical society, 73 (1951) 373-380.

[369] S. Brunauer, P.H. Emmett, E. Teller, Adsorption of gases in multimolecular layers, Journal of the American chemical society, 60 (1938) 309-319.

[370] R. Leboda, The chemical nature of adsorption centers in modified carbonsilica adsorbents prepared by the pyrolysis of alcohols, Chromatographia, 13 (1980) 703-708.

[371] S. Suganuma, K. Nakajima, M. Kitano, D. Yamaguchi, H. Kato, S. Hayashi, Hydrolysis of cellulose by amorphous carbon bearing $\mathrm{SO} 3 \mathrm{H}, \mathrm{COOH}$, and $\mathrm{OH}$ groups, Journal of the American Chemical Society, 130 (2008) 12787-12793.

[372] D. Koningsberger, R. Prins, X-ray absorption: principles, applications, techniques of EXAFS, SEXAFS, and XANES, (1988).

[373] S.J. Kim, Y.J. Park, E.J. Ra, K.K. Kim, K.H. An, Y.H. Lee, J.Y. Choi, C.H. Park, S.K. Doo, M.H. Park, Defect-induced loading of Pt nanoparticles on carbon 
nanotubes, Applied physics letters, 90 (2007) 023114.

[374] S.C. Benson, P. Cai, M. Colon, M.A. Haiza, M. Tokles, J.K. Snyder, Use of carboxylic acids as chiral solvating agents for the determination of optical purity of chiral amines by NMR spectroscopy, The Journal of Organic Chemistry, 53 (1988) 5335-5341.

[375] J. Post, J. Van Hooff, Acidity and activity of H-ZSM-5 measured with NH3-tpd and n-hexane cracking, Zeolites, 4 (1984) 9-14.

[376] Y. Liu, Y. Fang, X. Lu, Z. Wei, X. Li, Hydrogenation of nitrobenzene to paminophenol using $\mathrm{Pt} / \mathrm{C}$ catalyst and carbon-based solid acid, Chemical Engineering Journal, 229 (2013) 105-110.

[377] C.A. Antonyraj, J. Jeong, B. Kim, S. Shin, S. Kim, K.-Y. Lee, J.K. Cho, Selective oxidation of HMF to DFF using $\mathrm{Ru} / \gamma$-alumina catalyst in moderate boiling solvents toward industrial production, Journal of Industrial and Engineering Chemistry, 19 (2013) 1056-1059.

[378] A.C. Ferrari, J. Robertson, Interpretation of Raman spectra of disordered and amorphous carbon, Physical Review B, 61 (2000) 14095-14107.

[379] H. Yan, Y. Yang, D. Tong, X. Xiang, C. Hu, Catalytic conversion of glucose to 5-hydroxymethylfurfural over $\mathrm{SO} 42-/ \mathrm{ZrO} 2$ and $\mathrm{SO} 42-/ \mathrm{ZrO} 2-\mathrm{Al} 2 \mathrm{O} 3$ solid acid catalysts, Catalysis Communications, 10 (2009) 1558-1563.

[380] T. Liu, Z. Li, W. Li, C. Shi, Y. Wang, Preparation and characterization of biomass carbon-based solid acid catalyst for the esterification of oleic acid with methanol, Bioresource Technology, 133 (2013) 618-621.

[381] A.S. Amarasekara, L.D. Williams, C.C. Ebede, Mechanism of the dehydration of d-fructose to 5-hydroxymethylfurfural in dimethyl sulfoxide at 150\&\#xa0; ${ }^{\circ} \mathrm{C}$ : an NMR study, Carbohydrate Research, 343 (2008) 3021-3024.

[382] S. Caratzoulas, D.G. Vlachos, Converting fructose to 5hydroxymethylfurfural: a quantum mechanics/molecular mechanics study of the mechanism and energetics, Carbohydrate Research, 346 (2011) 664-672. 
[383] M.G. Mazzotta, D. Gupta, B. Saha, A.K. Patra, A. Bhaumik, M.M. AbuOmar, Efficient Solid Acid Catalyst Containing Lewis and Brønsted Acid Sites for the Production of Furfurals, ChemSusChem, 7 (2014) 2342-2350.

[384] K.-i. Shimizu, R. Uozumi, A. Satsuma, Enhanced production of hydroxymethylfurfural from fructose with solid acid catalysts by simple water removal methods, Catalysis Communications, 10 (2009) 1849-1853.

[385] Y. Yang, Z. Du, J. Ma, F. Lu, J. Zhang, J. Xu, Biphasic Catalytic Conversion of Fructose by Continuous Hydrogenation of HMF over a Hydrophobic Ruthenium Catalyst, ChemSusChem, 7 (2014) 1352-1356.

[386] A.A. Rosatella, S.P. Simeonov, R.F.M. Frade, C.A.M. Afonso, 5Hydroxymethylfurfural (HMF) as a building block platform: Biological properties, synthesis and synthetic applications, Green Chemistry, 13 (2011) 754793.

[387] H. Zhu, Q. Cao, C. Li, X. Mu, Acidic resin-catalysed conversion of fructose into furan derivatives in low boiling point solvents, Carbohydrate Research, 346 (2011) 2016-2018.

[388] Y. Roman-Leshkov, J.N. Chheda, J.A. Dumesic, Phase modifiers promote efficient production of hydroxymethylfurfural from fructose, Science, 312 (2006) 1933-1937.

[389] X. Sun, Y. Li, Colloidal Carbon Spheres and Their Core/Shell Structures with Noble-Metal Nanoparticles, Angewandte Chemie International Edition, 43 (2004) 597-601.

[390] S.H. Mushrif, S. Caratzoulas, D.G. Vlachos, Understanding solvent effects in the selective conversion of fructose to 5-hydroxymethyl-furfural: a molecular dynamics investigation, Physical Chemistry Chemical Physics, 14 (2012) 26372644.

[391] J.Y.G. Chan, Y. Zhang, Selective Conversion of Fructose to 5Hydroxymethylfurfural Catalyzed by Tungsten Salts at Low Temperatures, 
ChemSusChem, 2 (2009) 731-734.

[392] N.-T. Le, P. Lakshmanan, K. Cho, Y. Han, H. Kim, Selective oxidation of 5-hydroxymethyl-2-furfural into 2,5-diformylfuran over $\mathrm{VO} 2+$ and $\mathrm{Cu} 2+$ ions immobilized on sulfonated carbon catalysts, Applied Catalysis A: General, 464465 (2013) 305-312.

[393] Y. Guo, J. Chen, Bicomponent Assembly of VO2 and PolyanilineFunctionalized Carbon Nanotubes for the Selective Oxidation of Biomass-Based 5-Hydroxymethylfurfural to 2,5-Diformylfuran, ChemPlusChem, 80 (2015) $1760-1768$.

[394] Y. Gong, H. Wang, Z. Wei, L. Xie, Y. Wang, An Efficient Way To Introduce Hierarchical Structure into Biomass-Based Hydrothermal Carbonaceous Materials, ACS Sustainable Chemistry \& Engineering, 2 (2014) 2435-2441.

[395] B. Liu, Z. Zhang, K. Lv, K. Deng, H. Duan, Efficient aerobic oxidation of biomass-derived 5-hydroxymethylfurfural to 2, 5-diformylfuran catalyzed by magnetic nanoparticle supported manganese oxide, Applied Catalysis A: General, 472 (2014) 64-71.

[396] Y. Du, Y. Zhu, S. Xi, P. Yang, H.O. Moser, M.B.H. Breese, A. Borgna, XAFCA: a new XAFS beamline for catalysis research, Journal of Synchrotron Radiation, 22 (2015) 839-843.

[397] K.G. Sun, J.S. Chung, S.H. Hur, Durability Improvement of Pt/RGO Catalysts for PEMFC by Low-Temperature Self-Catalyzed Reduction, Nanoscale Research Letters, 10 (2015) 257.

[398] I. Sádaba, Y.Y. Gorbanev, S. Kegnæs, S.S.R. Putluru, R.W. Berg, A. Riisager, Catalytic Performance of Zeolite-Supported Vanadia in the Aerobic Oxidation of 5-hydroxymethylfurfural to 2,5-diformylfuran, ChemCatChem, 5 (2013) 284-293.

[399] C.A. Antonyraj, B. Kim, Y. Kim, S. Shin, K.-Y. Lee, I. Kim, J.K. Cho, Heterogeneous selective oxidation of 5-hydroxymethyl-2-furfural (HMF) into 
2,5-diformylfuran catalyzed by vanadium supported activated carbon in MIBK, extracting solvent for HMF, Catalysis Communications, 57 (2014) 64-68.

[400] F.L. Grasset, B. Katryniok, S. Paul, V. Nardello-Rataj, M. Pera-Titus, J.-M. Clacens, F. De Campo, F. Dumeignil, Selective oxidation of 5hydroxymethylfurfural to 2,5-diformylfuran over intercalated vanadium phosphate oxides, RSC Advances, 3 (2013) 9942-9948.

[401] B. Zambrano, E. Canizalez, P. Silva, A. Briceno, A bottom-up route for the preparation of novel hierarchical nanostructured hybrid molybdenum oxidehydrogel composites, New Journal of Chemistry, 35 (2011) 288-291.

[402] C. Avendano, A. Briceno, F.J. Mendez, J.L. Brito, G. Gonzalez, E. Canizales, R. Atencio, P. Dieudonne, Novel MoO2/carbon hierarchical nano/microcomposites: synthesis, characterization, solid state transformations and thiophene HDS activity, Dalton Transactions, 42 (2013) 2822-2830.

[403] T.H. Chiang, M.Y. Chen, M.H. Li, M.Y. Yen, Synthesis and structural characterization of $\alpha-\mathrm{MoO} 3$ from molybdic acid wastewater, Journal of Materials Science, 48 (2013) 6994-7003.

[404] N.X. Jingxing Chen, Tianxiang Zhou, Sanxiang Tan, Fengping Jiang, Dingsheng Yuan*, Mesoporous Carbon Spheres: Synthesis, Characterization and Supercapacitance International Journal of Electrochemical Science, 4 (2009) 111. [405] S. Song, S. Jiang, R. Rao, H. Yang, A. Zhang, Bicomponent VO2defects/MWCNT catalyst for hydroxylation of benzene to phenol: Promoter effect of defects on catalytic performance, Applied Catalysis A: General, 401 (2011) 215-219.

[406] A. Wang, Y. Fang, L. Long, Y. Song, W. Yu, W. Zhao, M.P. Cifuentes, M.G. Humphrey, C. Zhang, Facile Synthesis and Enhanced Nonlinear Optical Properties of Porphyrin-Functionalized Multi-Walled Carbon Nanotubes, Chemistry - A European Journal, 19 (2013) 14159-14170.

[407] J. Dou, H.C. Zeng, Preparation of Mo-Embedded Mesoporous Carbon 
Microspheres for Friedel-Crafts Alkylation, The Journal of Physical Chemistry C, 116 (2012) 7767-7775.

[408] A. Auroux, A. Gervasini, Microcalorimetric study of the acidity and basicity of metal oxide surfaces, The Journal of Physical Chemistry, 94 (1990) 6371-6379.

[409] Z.-Z. Yang, J. Deng, T. Pan, Q.-X. Guo, Y. Fu, A one-pot approach for conversion of fructose to 2,5-diformylfuran by combination of Fe3O4-SBASO3H and K-OMS-2, Green Chemistry, 14 (2012) 2986-2989.

[410] G.A. Halliday, R.J. Young, V.V. Grushin, One-Pot, Two-Step, Practical Catalytic Synthesis of 2,5-Diformylfuran from Fructose, Organic Letters, 5 (2003) 2003-2005. 


\section{Appendix}

\section{Journal publications}

[1] J. Zhao, Y. Dai, Y. Yan, C. He, Y. Yang*, Selective aerobic oxidation of 5-hydroxymethyl-2-furfural into 2, 5-diformylfuran over V-embedded mesoporous carbon microspheres, (2016-prepared).

[2] J. Zhao, L. Bai, J. Huang, Y. Yan, Y. Yang*, Molybdenum trioxide Supported on Carbon Sphere for One-Step Approach to 2,5-Diformylfuran from Fructose, (2016-prepared).

[3] Y. Yan, K. Li, L. Thia, Y. Dai, J. Zhao, X. Chen, Y. Yang, J.-M. Lee, hydrothermally driven three-dimensional evolution of mesoporous hierarchical europium oxide hydrangea microspheres for non-enzymatic sensors of hydrogen peroxide detection, Environmental Science: Nano, (2016).

[4] H. Lv, H. Zhang, J. Zhao, G. Ji, Y. Du, Achieving excellent bandwidth absorption by a mirror growth process of magnetic porous polyhedron structures, Nano Research, 1-10.

[5] C. He, J. Zhao, Y. Yang, J.-Y. Wang, Multiscale characteristics dynamics of hydrochar from hydrothermal conversion of sewage sludge under sub-and near-critical water, Bioresource technology, 211 (2016) 486-493.

[6] J. Zhao, C. Zhou, C. He, Y. Dai, X. Jia, Y. Yang, Efficient dehydration of fructose to 5-hydroxymethylfurfural over sulfonated carbon sphere solid acid catalysts, Catalysis Today, 264 (2016) 123-130.

[7] K. Li, Z. Yang, J. Zhao, J. Lei, X. Jia, S.H. Mushrif, Y. Yang, Mechanistic and kinetic studies on biodiesel production catalyzed by an efficient pyridinium based ionic liquid, Green Chemistry, 17 (2015) 4271-4280. 